\title{
Rigged Configurations and Kashiwara Operators ${ }^{\star}$
}

Reiho SAKAMOTO

Department of Physics, Tokyo University of Science, Kagurazaka, Shinjuku, Tokyo, Japan

E-mail: reiho@rs.tus.ac.jp, reihosan@08.alumni.u-tokyo.ac.jp

URL: https://sites.google.com/site/affinecrystal

Received April 02, 2013, in final form February 28, 2014; Published online March 23, 2014

http://dx.doi.org/10.3842/SIGMA.2014.028

Abstract. For types $A_{n}^{(1)}$ and $D_{n}^{(1)}$ we prove that the rigged configuration bijection intertwines the classical Kashiwara operators on tensor products of the arbitrary KirillovReshetikhin crystals and the set of the rigged configurations.

Key words: crystal bases; rigged configurations; quantum affine algebras; box-ball systems

2010 Mathematics Subject Classification: 17B37; 05E10; 82B23

Dedicated to Professor Anatol N. Kirillov with admiration

\section{Contents}

1 Introduction $\quad 3$

2 Background on crystals and tableaux $\quad \mathbf{5}$

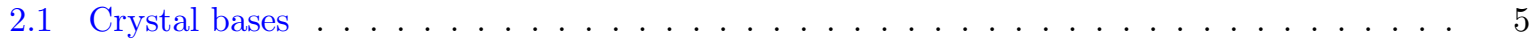

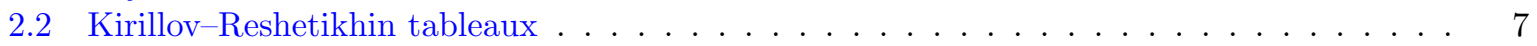

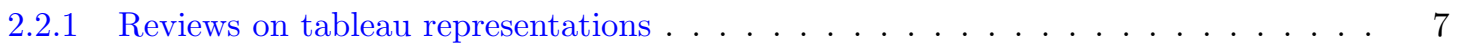

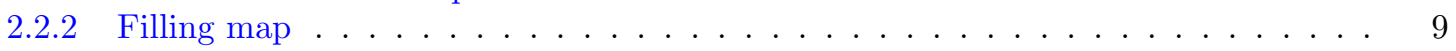

3 Rigged configurations and the main bijection $\quad 11$

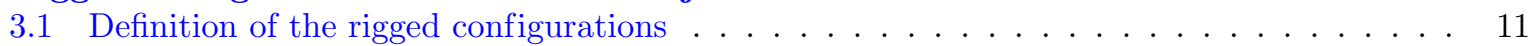

3.2 Convexity relations of the vacancy numbers and their applications . . . . . . . . . 13

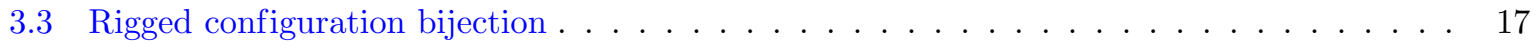

4 Rigged configurations and the Kashiwara operators $\quad 21$

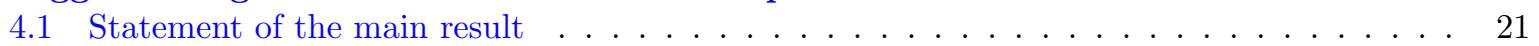

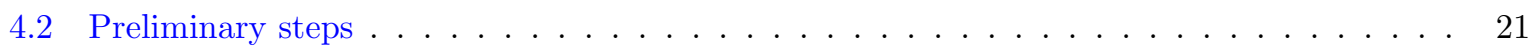

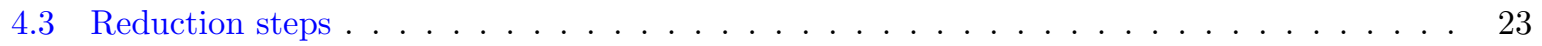

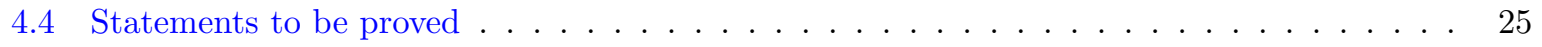

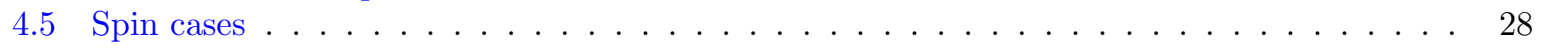

5 Proof of Proposition $4.7 \quad 30$

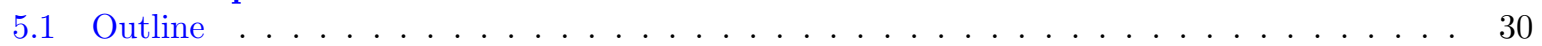

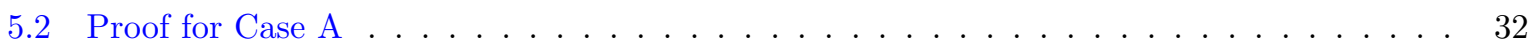

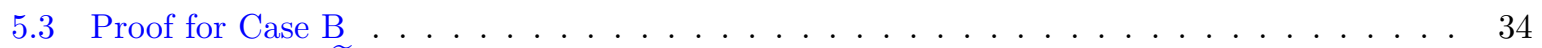

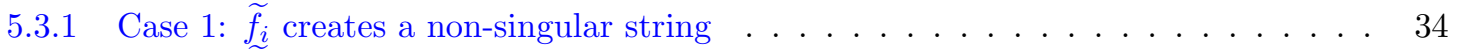

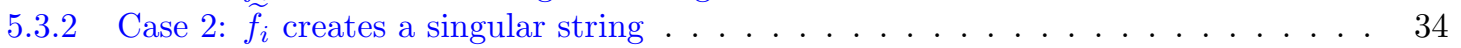

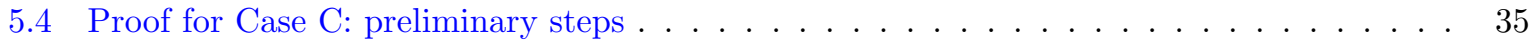

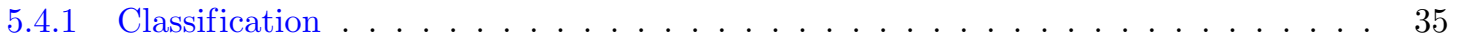

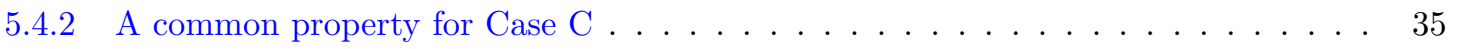

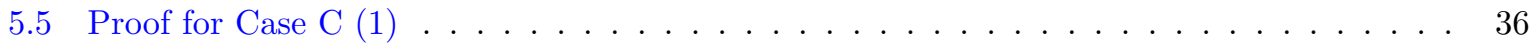

${ }^{\star}$ This paper is a contribution to the Special Issue in honor of Anatol Kirillov and Tetsuji Miwa. The full collection is available at http://www.emis.de/journals/SIGMA/InfiniteAnalysis2013.html 


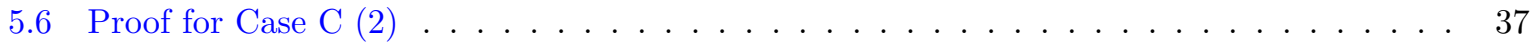

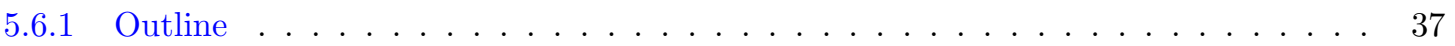

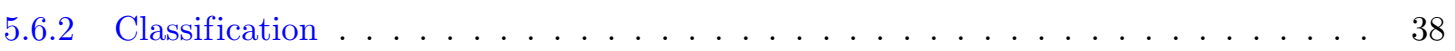

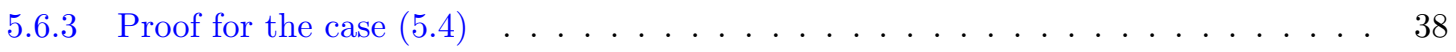

5.6 .4 Proof for the case $(5.5) \ldots \ldots \ldots \ldots \ldots \ldots \ldots \ldots \ldots$

5.7 Proof for Case $\mathrm{C}(3) \ldots \ldots \ldots \ldots \ldots \ldots$

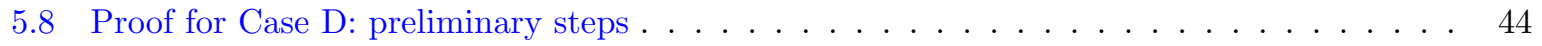

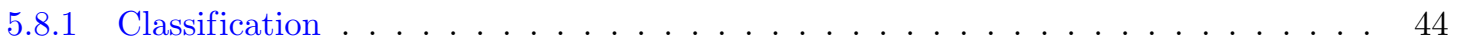

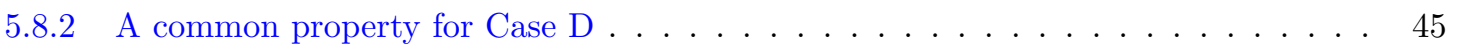

5.9 Proof for Case D $(1) \ldots \ldots \ldots \ldots \ldots \ldots \ldots$

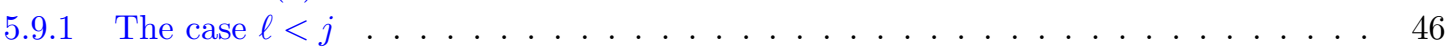

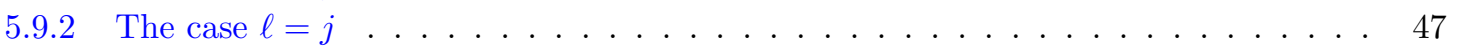

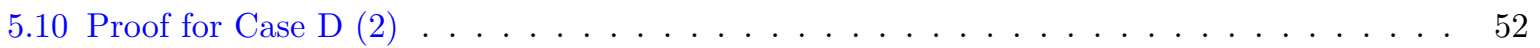

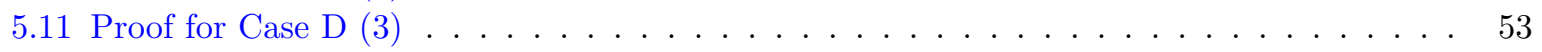

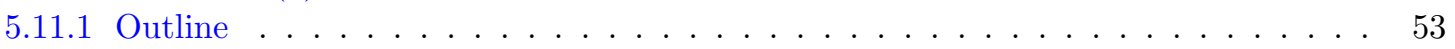

5.11 .2 Proof of Proposition 5.30 for the case $\ell^{(i-1)}<\ell \ldots \ldots \ldots \ldots 5$

5.11 .3 Proof of Proposition 5.30 for the case $\ell^{(i-1)}=\ell \ldots \ldots \ldots \ldots$. . . . . . . 57

5.12 Proof for Case D $(4) \ldots \ldots \ldots \ldots \ldots \ldots$

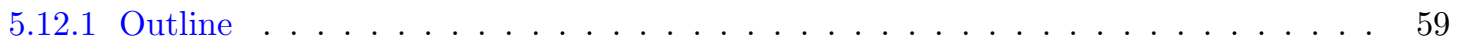

5.12 .2 Proof of Proposition 5.39 for the case $\ell^{(i-1)}<\ell \ldots \ldots \ldots \ldots$

5.12 .3 Proof of Proposition 5.39 for the case $\ell^{(i-1)}=\ell \ldots \ldots \ldots \ldots \ldots$. . . . . . 61

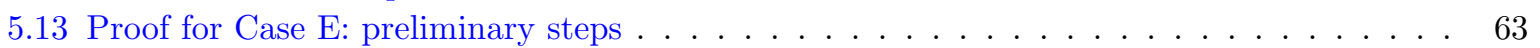

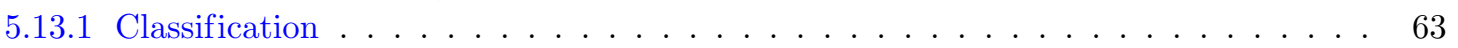

5.13 .2 A common property for Case E . . . . . . . . . . . . . . . 63

5.14 Proof for Case E $(1) \ldots \ldots \ldots \ldots \ldots \ldots \ldots \ldots$

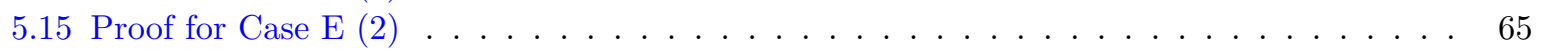

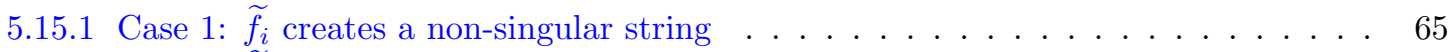

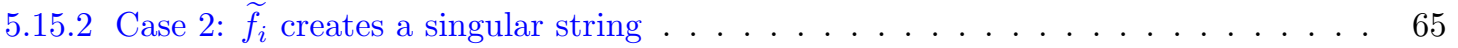

5.16 Proof for Case E $(3) \ldots \ldots \ldots \ldots \ldots \ldots \ldots$

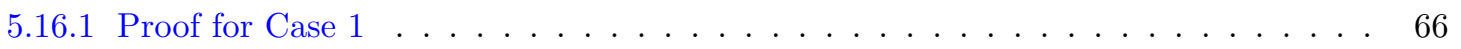

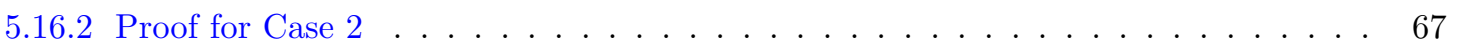

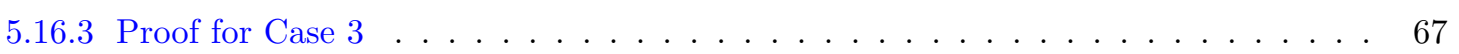

5.17 Proof for Case E $(4) \ldots \ldots \ldots \ldots \ldots \ldots \ldots \ldots$

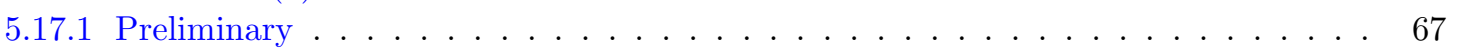

5.17 .2 Proof for Case $1 \ldots \ldots \ldots \ldots \ldots \ldots$

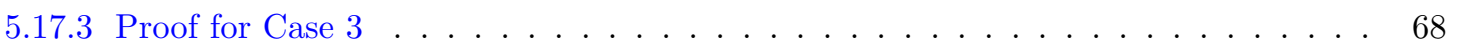

5.18 Proof for Case E $(5) \ldots \ldots \ldots \ldots \ldots \ldots \ldots \ldots$

6 Proof of Proposition 4.8: $\tilde{f}_{i}$ version $\quad 69$

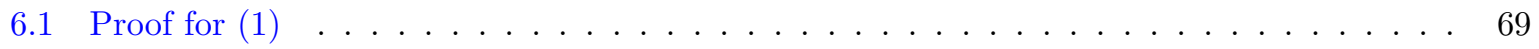

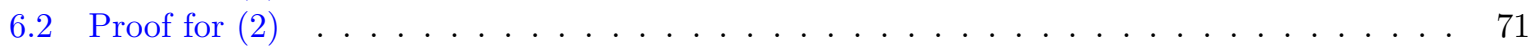

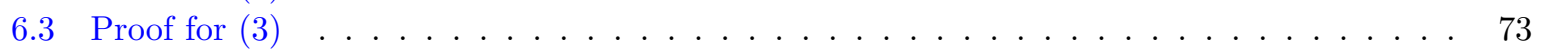

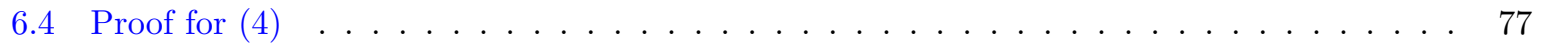

$\begin{array}{lll}7 & \text { Proof of Proposition 4.8: } \widetilde{e}_{i} \text { version } & \mathbf{7 7}\end{array}$

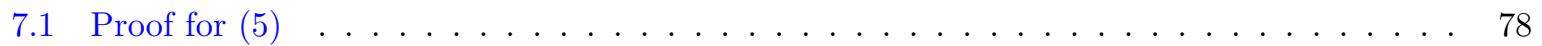

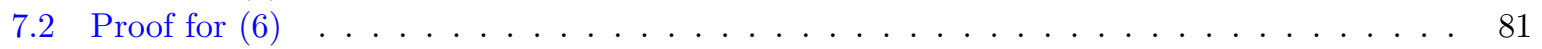

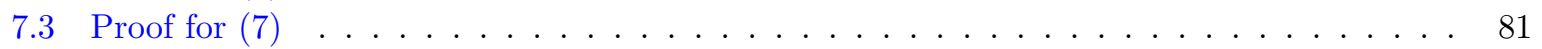

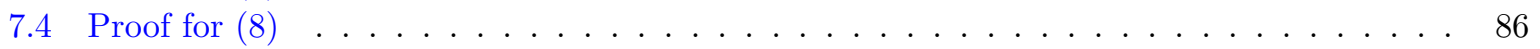

8 Comments on Deka-Schilling's work $\quad 86$

$\begin{array}{ll}\text { References } & 87\end{array}$ 


\section{Introduction}

A search of a natural presentation of the basis for representations of infinite dimensional algebras sometimes reveals an unexpected and intriguing connection with other models of mathematical physics. Take the Feigin-Fuchs representation of the Virasoro algebra [7] as an example. In this case, a cerebrated integrable quantum many body problem called the Calogero-Sutherland model [35, 36] plays the role. Indeed, it is conjectured in [30] and partially proved in [3] that the excited states of the Calogero-Sutherland model (usually called the Jack symmetric functions) behave particularly nicely as a basis for the Feigin-Fuchs module. For example, matrix elements of the Virasoro generators with respect to the Jack basis become factorized rational functions whose major part consisting of degree one polynomials of positive integer coefficients which admit a transparent combinatorial interpretation. This phenomenon seems not accidental since the theory includes Mimachi and Yamada's finding [22] which claims that the singular vectors of the Virasoro algebra coincide with special cases of the Jack symmetric functions. See, for example, $[1,2,5,6,8]$ for recent developments on the subject.

The purpose of the present paper is to pursue a parallel investigation for the settings of the crystal bases [14] of an important class of finite dimensional representations (called the KirillovReshetikhin modules) of the quantum affine algebras. We call the corresponding crystal the Kirillov-Reshetikhin (KR) crystal. In this case, the corresponding physical model is the boxball system. The box-ball system is a prototypical example of the ultradiscrete (or tropical) soliton system whose simplest example was discovered by Takahashi and Satsuma [37]. For our purpose, an important aspect of the box-ball system is that the dynamics of the model is determined by the combinatorial $R$-matrices for the KR crystal [9, 10]. This formalism allows us to define the box-ball system for any tensor products of the KR crystals for arbitrary quantum affine algebras (see, for example, [11, 12]). See [29] for an introductory review on studies on the box-ball systems.

Recently, a complete set of the action and angle variables of the type $A_{n}^{(1)}$ box-ball systems is found to be identical to the set of the rigged configurations [20]. The rigged configurations are combinatorial objects discovered by an insightful analysis of the Bethe ansatz for quantum integrable models $[17,18]$. Originally the rigged configurations are used to prove a combinatorial identity for the Kostka-Foulkes polynomials called the Fermionic formula as well as to give a description of the branching coefficients of finite dimensional representations of the quantum affine algebras with respect to the corresponding finite dimensional subalgebras. In such context the rigged configurations are labels of representations and thus naturally correspond to the classically highest weight tensor products of the KR crystals. On the other hand, since the boxball system is defined for not necessarily highest weight tensor products, we need to consider generalized rigged configurations corresponding to any tensor products.

Therefore it is natural to introduce the Kashiwara operators on the set of the rigged configurations. Let $I$ be the set of the Dynkin nodes of a quantum affine algebra $\mathfrak{g}$ following Kac's convention [13] and set $I_{0}=I \backslash\{0\}$. In [32] Schilling gave a definition of the classical Kashiwara operators $\widetilde{e}_{i}$ and $\widetilde{f}_{i}\left(i \in I_{0}\right)$ for simply laced algebras and proved that the crystal structure on the rigged configurations is actually isomorphic to the one on the tensor products of the KR crystals by using Stembridge's local characterization of crystals. In Section 3.2 of the present paper, we provide a direct proof of the fact that the classical Kashiwara operators on the set of the rigged configurations of types $A_{n}^{(1)}$ and $D_{n}^{(1)}$ indeed satisfy the axiom of the crystals.

The crux of the rigged configuration theory is the so called rigged configuration bijection which gives an one to one correspondence between the elements of tensor products of the KR crystals and the rigged configurations:

$\Phi$ : rigged configurations $\longmapsto$ tensor products. 
The algorithm of $\Phi$ is a rather complicated combinatorial procedure which is explained in Section $3.3^{1}$. Nevertheless it is known that the map $\Phi$ has a lot of nice properties and seems to have a deep mathematical origin. For example, one of the most important properties of the rigged configuration bijection is that we can regard the algorithm $\Phi$ as a general form of the combinatorial $R$-matrices ([19] for type $A_{n}^{(1)}$ case and $[31,34]$ for type $D_{n}^{(1)}$ case). See Remark 3.19 for more precise meanings. This property is the basis for providing the action and angle variables of the box-ball systems $[20,21]$. Moreover, as an application of the relationship with the box-ball system we have a representation theoretical interpretation of the algorithm of $\Phi^{-1}$ for type $A_{n}^{(1)}$ [28], though the full understanding of the rigged configurations themselves is yet to be seen.

The main result of the present paper is the proof of the following compatibility of the rigged configuration bijection and the classical Kashiwara operators

$$
\left[\widetilde{e}_{i}, \Phi\right]=0, \quad\left[\widetilde{f}_{i}, \Phi\right]=0, \quad i \in I_{0}
$$

for $\mathfrak{g}=A_{n}^{(1)}$ and $D_{n}^{(1)}$ (see Theorem 4.1) under the assumption that the bijection $\Phi$ is welldefined. Here some remarks are in order.

- In Appendix C of the preprint version of [4], Deka and Schilling asserted the relation (1.1) for type $A_{n}^{(1)}$. However, according to the author's opinion, they did not finish their proof (see Section 8). Given the fundamental importance of the problem, it seems appropriate to prove the result completely for type $A_{n}^{(1)}$ also. Indeed, the proof for type $A_{n}^{(1)}$ is a part of the proof for type $D_{n}^{(1)}$ and easy to extract from it ${ }^{2}$.

- The proof of (1.1) is direct and does not depend on other results.

Since our approach is direct, we expect that our result paves the way to deal with the rigged configuration bijection for arbitrary quantum affine algebras. Currently the following cases are established:

- All cases of type $A_{n}^{(1)}[17,18,19]$.

- Special cases of type $D_{n}^{(1)}[24,31,34]$ (see the comments after Conjecture 3.17).

- Tensor products of the vector representations of arbitrary non-exceptional quantum affine algebras [27] as well as of type $E_{6}^{(1)}[26]$.

In all cases the algorithms for the rigged configuration bijection share many common features. We expect that the rigged configuration bijection as well as all major properties will extend to the arbitrary quantum affine algebras.

We expect that such an extension of the rigged configuration theory will provide meaningful insights for both mathematical physics side and mathematical side which are not easily seen by other methods. One of the supporting evidences for our expectation is a bijection constructed in [23]. In that paper, we constructed a combinatorial bijection between the rigged configurations for arbitrary non-exceptional quantum affine algebras (under certain restrictions on ranks) and the set of pairs of a type $A_{n}^{(1)}$ rigged configuration and a Littlewood-Richardson tableau. Remarkably, the construction is uniform for all types of algebras and the LittlewoodRichardson tableaux naturally appear as the recording tableaux of the algorithm. We expect

\footnotetext{
${ }^{1}$ For the most general tensor products $B^{r_{1}}, s_{1} \otimes B^{r_{2}, s_{2}} \otimes \cdots \otimes B^{r_{L}, s_{L}}$ of type $D_{n}^{(1)}$, a proof of the well-definedness of the bijection $\Phi$ is the subject of [25]. However we will not assume this fact in the present paper since the paper [25] is yet not available for the public. After Conjecture 3.17, we summarize the known cases of this result.

${ }^{2}$ Ignore all arguments related with the parameter $\ell_{(a)}$ by formally setting $\ell_{(a)}=\infty$. See Section 3.3 for the notation.
} 
that the bijection coincides with (and generalizes) a canonical Dynkin diagram automorphism (see Remark 3.2 of [23]).

Another supporting evidence is a numerical comparison of the rigged configuration bijection [27] for the vector representations of arbitrary non-exceptional quantum affine algebras and the corresponding combinatorial $R$-matrices (see Section 3 and subsequent sections of [20]). The conjecture provided there claims that seemingly complicated algorithms presented in [27] with many case by case definition possess uniform description in terms of the corresponding combinatorial $R$-matrices. In fact, we expect that the rigged configuration bijection gives a general form of the combinatorial $R$-matrices for general quantum affine algebras and thus provides action and angle variables for the corresponding box-ball systems. Finally, it should be mentioned that in [24] we see that the affine crystal structure of type $D_{n}^{(1)}$ is essentially governed by the rigged configurations. Therefore we expect that the construction of the rigged configuration bijection for general cases should be an intriguing and meaningful future research topic.

This paper is organized as follows. In Section 2, we collect necessary facts from the crystal bases and the tableau representations. In Section 3, we give the definition of the rigged configurations and the rigged configuration bijection and explain their basic properties (convexity relation of the vacancy numbers). We also introduce the classical Kashiwara operators on the rigged configurations and show that they satisfy the axiom of the classical crystals. In Section 4 we give the statement of the main result and clarify what are the essential points to be shown. Main proofs are given in Sections 5,6 and 7. During the proof we have tried to avoid unnecessarily long arguments. Nevertheless we have to deal with many subtle cases and some of them look very special. Thus some readers might wonder whether such case does exist and is worthy of careful analysis. In order to clarify that point, we include several examples for seemingly subtle cases.

\section{Background on crystals and tableaux}

\subsection{Crystal bases}

Let us briefly recall some definitions about the crystal bases theory of Kashiwara [14]. For more detailed introduction, see, for example, [15]. Let $\mathfrak{g}$ be an affine Kac-Moody algebra, $\mathfrak{g}^{\prime}$ be its derived subalgebra, $\mathfrak{g}_{0}$ be the corresponding finite dimensional simple Lie algebra obtained by removing the 0 node of the Dynkin diagram of $\mathfrak{g}$. Here we follow Kac' convention [13] for the labeling of the Dynkin nodes and denote by $I$ the vertex set of the Dynkin diagram of $\mathfrak{g}$ and $I_{0}:=I \backslash\{0\}$. Let $U_{q}(\mathfrak{g}), U_{q}^{\prime}(\mathfrak{g})$ and $U_{q}\left(\mathfrak{g}_{0}\right)$ be the quantized universal enveloping algebra corresponding to $\mathfrak{g}, \mathfrak{g}^{\prime}$ and $\mathfrak{g}_{0}$, respectively. In this paper we will mainly focus on the case $\mathfrak{g}=D_{n}^{(1)}$ which has the following Dynkin diagram

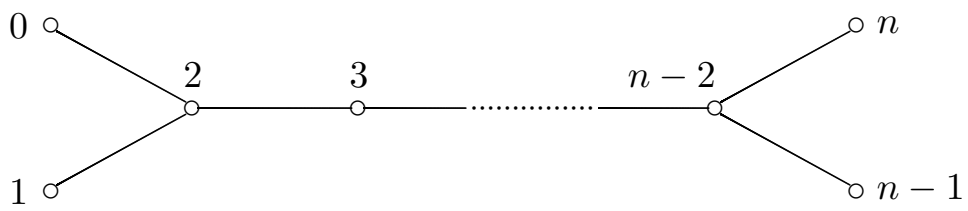

Let $\alpha_{i}, h_{i}, \Lambda_{i}(i \in I)$ be the simple roots, simple coroots and fundamental weights of $\mathfrak{g}, \bar{\Lambda}_{i}$ $\left(i \in I_{0}\right)$ be the fundamental weights of $\mathfrak{g}_{0}$. In the present case $\mathfrak{g}_{0}=D_{n}$, the simple roots are

$$
\alpha_{i}=\epsilon_{i}-\epsilon_{i+1}, \quad 1 \leq i \leq n-1, \quad \alpha_{n}=\epsilon_{n-1}+\epsilon_{n},
$$

and the fundamental weights are

$$
\begin{aligned}
& \bar{\Lambda}_{i}=\epsilon_{1}+\cdots+\epsilon_{i}, \quad 1 \leq i \leq n-2, \\
& \bar{\Lambda}_{n-1}=\left(\epsilon_{1}+\cdots+\epsilon_{n-1}-\epsilon_{n}\right) / 2, \quad \bar{\Lambda}_{n}=\left(\epsilon_{1}+\cdots+\epsilon_{n-1}+\epsilon_{n}\right) / 2,
\end{aligned}
$$


where $\epsilon_{i} \in \mathbb{Z}^{n}$ is the $i$ th standard unit vector. Let $Q, Q^{\vee}, P$ be the root, coroot and weight lattices of $\mathfrak{g}$. Let $\langle\cdot, \cdot\rangle: Q^{\vee} \otimes P \rightarrow \mathbb{Z}$ be the pairing such that $\left\langle h_{i}, \Lambda_{j}\right\rangle=\Lambda_{j}\left(h_{i}\right)=\delta_{i j}$. Note that we have $\left\langle h_{i}, \alpha_{j}\right\rangle=\alpha_{j}\left(h_{i}\right)=A_{i j}$ where $A_{i j}$ is the Cartan matrix of $\mathfrak{g}=D_{n}^{(1)}$.

Now we give the axiomatic definition of $U_{q}^{\prime}(\mathfrak{g})$-crystals.

Definition 2.1. $U_{q}^{\prime}(\mathfrak{g})$-crystal is a nonempty set $B$ equipped with maps wt : $B \rightarrow P, \varepsilon_{i}, \varphi_{i}$ : $B \rightarrow \mathbb{Z} \cup\{-\infty\}$ and the Kashiwara operators $\widetilde{e}_{i}, \widetilde{f}_{i}: B \rightarrow B \cup\{0\}$ for all $i \in I$ such that

(1) $\left\langle h_{i}, \operatorname{wt}(b)\right\rangle=\varphi_{i}(b)-\varepsilon_{i}(b)$,

(2) $\operatorname{wt}\left(\widetilde{e}_{i}(b)\right)=\operatorname{wt}(b)+\alpha_{i}$ if $\widetilde{e}_{i}(b) \in B$,

(3) $\operatorname{wt}\left(\widetilde{f}_{i}(b)\right)=\operatorname{wt}(b)-\alpha_{i}$ if $\tilde{f}_{i}(b) \in B$,

(4) $\varepsilon_{i}\left(\widetilde{e}_{i}(b)\right)=\varepsilon_{i}(b)-1, \varphi_{i}\left(\widetilde{e}_{i}(b)\right)=\varphi_{i}(b)+1$ if $\widetilde{e}_{i}(b) \in B$,

(5) $\varepsilon_{i}\left(\tilde{f}_{i}(b)\right)=\varepsilon_{i}(b)+1, \varphi_{i}\left(\widetilde{f}_{i}(b)\right)=\varphi_{i}(b)-1$ if $\tilde{f}_{i}(b) \in B$,

(6) for $b, b^{\prime} \in B, \widetilde{f}_{i}(b)=b^{\prime}$ if and only if $\widetilde{e}_{i}\left(b^{\prime}\right)=b$,

(7) if $\varphi_{i}(b)=-\infty$ for $b \in B$, then $\widetilde{e}_{i}(b)=\widetilde{f}_{i}(b)=0$.

In this paper, we only consider the case when the maps $\varepsilon_{i}, \varphi_{i}$ are defined by

$$
\varepsilon_{i}(b)=\max \left\{m \geq 0 \mid \widetilde{e}_{i}^{m}(b) \neq 0\right\}, \quad \varphi_{i}(b)=\max \left\{m \geq 0 \mid \widetilde{f}_{i}^{m}(b) \neq 0\right\}
$$

for $b \in B$. In particular, we have $0 \leq \varepsilon_{i}(b), \varphi_{i}(b)<\infty$ for all $i \in I$ and $\underset{\widetilde{b}}{b} \in B$. Note that the crystals with these conditions are called the regular crystals. If we have $\widetilde{f}_{i}(b)=b^{\prime}$ for $b, b^{\prime} \in B$, we write an arrow with color (or label) $i$ from $b$ to $b^{\prime}$. In this way, the crystal $B$ can be regarded as the colored oriented graph whose vertices are the elements of $B$. We call such graph the crystal graph.

One of the nice properties of the crystal bases is that it behaves nicely with respect to the tensor product. Let $B_{2} \otimes B_{1}$ be the tensor product of two crystals $B_{1}$ and $B_{2}$. As the set, it coincides with the Cartesian product $B_{2} \times B_{1}$. The action of the Kashiwara operators $\widetilde{e}_{i}, \widetilde{f}_{i}$ on an element $b_{2} \otimes b_{1} \in B_{2} \otimes B_{1}$ is given explicitly as follows:

$$
\begin{aligned}
& \widetilde{e}_{i}\left(b_{2} \otimes b_{1}\right)=\left\{\begin{array}{lll}
\widetilde{e}_{i}\left(b_{2}\right) \otimes b_{1} & \text { if } \quad \varepsilon_{i}\left(b_{2}\right)>\varphi_{i}\left(b_{1}\right), \\
b_{2} \otimes \widetilde{e}_{i}\left(b_{1}\right) & \text { if } \quad \varepsilon_{i}\left(b_{2}\right) \leq \varphi_{i}\left(b_{1}\right),
\end{array}\right. \\
& \widetilde{f}_{i}\left(b_{2} \otimes b_{1}\right)=\left\{\begin{array}{lll}
\widetilde{f}_{i}\left(b_{2}\right) \otimes b_{1} & \text { if } \varepsilon_{i}\left(b_{2}\right) \geq \varphi_{i}\left(b_{1}\right), \\
b_{2} \otimes \widetilde{f}_{i}\left(b_{1}\right) & \text { if } \quad \varepsilon_{i}\left(b_{2}\right)<\varphi_{i}\left(b_{1}\right),
\end{array}\right.
\end{aligned}
$$

where the result is declared to be 0 if either of its tensor factors are 0 . We also have $\operatorname{wt}\left(b_{2} \otimes b_{1}\right)=$ $\mathrm{wt}\left(b_{2}\right)+\mathrm{wt}\left(b_{1}\right)$.

Remark 2.2. We use the opposite of the Kashiwara's tensor product convention.

In order to deal with multiple tensor products, it is convenient to use the signature rule. Let $B$ be the tensor product of crystals $B=B_{L} \otimes \cdots \otimes B_{2} \otimes B_{1}$. For $i \in I$ and $b=b_{L} \otimes \cdots \otimes b_{2} \otimes b_{1} \in B$, the $i$-signature of $b$ is the following sequence of the symbols + and -

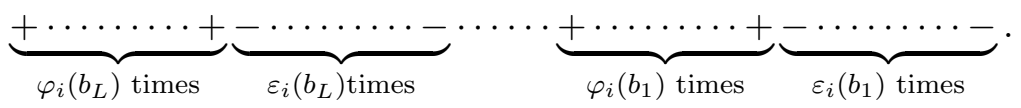

The reduced $i$-signature of $b$ is obtained by removing subsequence -+ of the $i$-signature of $b$ repeatedly until it becomes the following form

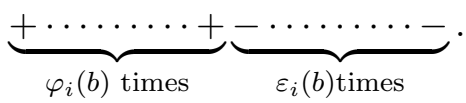


By using this information the action of $\widetilde{e}_{i}$ and $\widetilde{f}_{i}$ on $b$ can be described as follows. If $\varepsilon_{i}(b)=0$, then $\widetilde{e}_{i}(b)=0$. Otherwise

$$
\widetilde{e}_{i}\left(b_{L} \otimes \cdots \otimes b_{1}\right)=b_{L} \otimes \cdots \otimes b_{j+1} \otimes \widetilde{e}_{i}\left(b_{j}\right) \otimes b_{j-1} \otimes \cdots \otimes b_{1},
$$

where the leftmost - in the reduced $i$-signature of $b$ comes from $b_{j}$. Similarly, if $\varphi_{i}(b)=0$, then $\widetilde{f}_{i}(b)=0$. Otherwise

$$
\tilde{f}_{i}\left(b_{L} \otimes \cdots \otimes b_{1}\right)=b_{L} \otimes \cdots \otimes b_{j+1} \otimes \tilde{f}_{i}\left(b_{j}\right) \otimes b_{j-1} \otimes \cdots \otimes b_{1},
$$

where the rightmost + in the reduced $i$-signature of $b$ comes from $b_{j}$. Finally the actions of $\widetilde{e}_{i}$ and $\widetilde{f}_{i}$ on each tensor factor $b_{j}$ are described by tableau representations of $b_{j}$ described in the sequel.

\subsection{Kirillov-Reshetikhin tableaux}

In order to perform explicit manipulations on the crystal bases, it is convenient to use an explicit realization of the elements of crystals. For the present purpose, it is more convenient to use a new kind of tableau representation which we call the Kirillov-Reshetikhin (KR) tableaux [24] than the usual Kashiwara-Nakashima (KN) tableaux [16].

\subsubsection{Reviews on tableau representations}

Let us briefly recall tableau representations of crystals. Let $B(\Lambda)$ be the crystal associated to the highest weight representation of highest weight $\Lambda$ of $U_{q}\left(\mathfrak{g}_{0}\right)$. Then in the present case $\mathfrak{g}=D_{n}^{(1)}$, the $U_{q}^{\prime}(\mathfrak{g})$ crystal $B^{r, s}$ has the following decomposition under the restriction to its finite dimensional subalgebra $U_{q}\left(\mathfrak{g}_{0}\right)$. If $r<n-1$, we have

$$
B^{r, s} \simeq \bigoplus_{\lambda} B(\lambda) \quad \text { as } \mathfrak{g}_{0} \text { crystals }
$$

and if $r=n-1, n$, we have

$$
B^{r, s} \simeq B\left(s \bar{\Lambda}_{r}\right) \quad \text { as } \mathfrak{g}_{0} \text { crystals. }
$$

Here in the first case $r<n-1, \lambda$ in the summation are determined as follows. For this we identify $\lambda=\sum_{i} k_{i} \bar{\Lambda}_{i}$ with the Young diagram which has $k_{i}$ columns of height $i$ for all $i$. Then one of $\lambda$ in decomposition (2.3) is shape $\left(s^{r}\right)$ rectangular diagram and the remaining $\lambda$ are obtained by removing the vertical dominoes $\boxminus$ from $\left(s^{r}\right)$ rectangular diagram in all possible ways. Then if $b \in B^{r, s}$ belongs to some $B(\lambda)$ in the right hand side of the decomposition (2.3) or (2.4), then the KN tableau representation of $b$ has shape $\lambda$.

KN tableaux for $\boldsymbol{B}^{\boldsymbol{r}, \boldsymbol{s}}$ when $\boldsymbol{r} \leq \boldsymbol{n}-\mathbf{2}$. For $J \subset I$, we say $b$ is $J$-highest element if we have $\widetilde{e}_{i}(b)=0$ for all $i \in J$. Suppose that $u_{\lambda} \in B^{r, s}(r \leq n-2)$ is the $I_{0}$-highest element of $B(\lambda)$ which appears in the decomposition (2.3). Then the corresponding KN tableau is obtained by filling the letter $j$ to all the boxes at $j$ th row of the Young diagram $\lambda$. In order to obtain the other tableaux, we define the action of the Kashiwara operators on tableaux as follows. The crystal graph of $B^{1,1}$ for $\mathfrak{g}_{0}=D_{n}$ is as follows

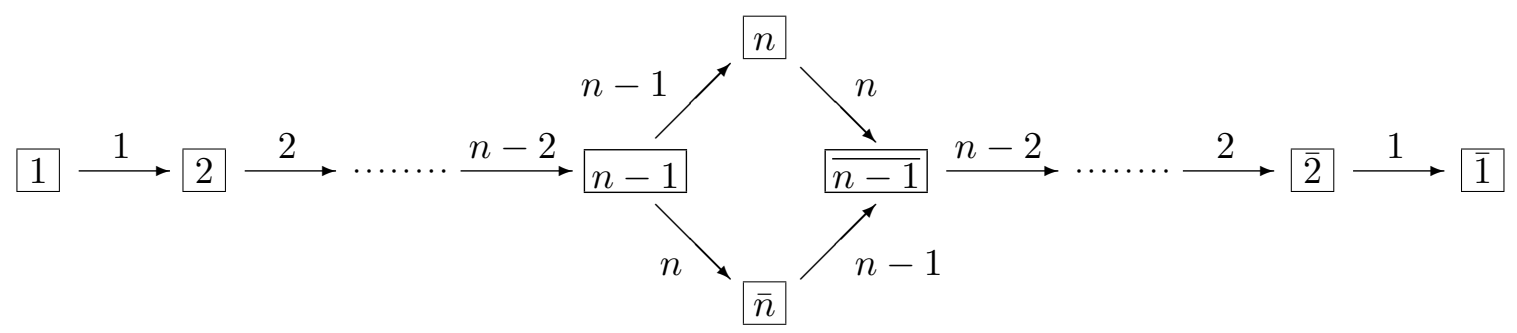


Weight of each vertex is $\operatorname{wt}(i)=\epsilon_{i}$ and $\mathrm{wt}(\bar{i})=-\epsilon_{i}$, respectively. For a given tableau $t \in B^{r, s}(r \leq n-2)$,

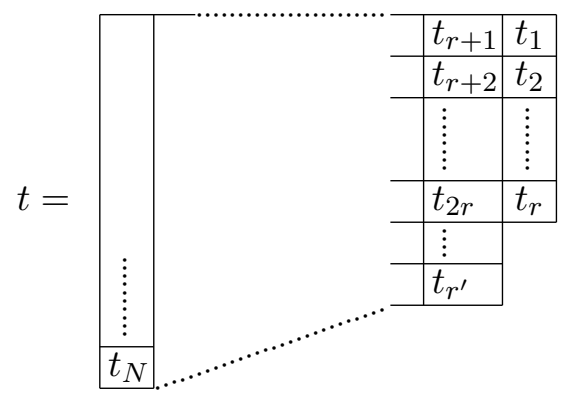

we introduce the so-called Japanese reading word and regard $t$ as the element of $\left(B^{1,1}\right)^{\otimes N}$ as follows

$$
t \longmapsto t_{N} \otimes \cdots \otimes t_{2} \otimes t_{1} \in\left(B^{1,1}\right)^{\otimes N} .
$$

Via this identification we can introduce the Kashiwara operators on tableaux and obtain the $\mathrm{KN}$ tableau representation for the general case.

KN tableaux for $\boldsymbol{B}^{\boldsymbol{n}, 2 s+1}$. Let us consider the basic case $B^{n, 1} \simeq B\left(\bar{\Lambda}_{n}\right)$. This is the so-called spin representation of dimension $2^{n-1}$ whose basis are $\left(s_{1}, s_{2}, \ldots, s_{n}\right)$ where $s_{i}= \pm$ and $s_{1} s_{2} \cdots s_{n}=1$. The classical Kashiwara operators act on each basis as follows:

$$
\begin{aligned}
& \widetilde{e}_{i}\left(s_{1}, \ldots, s_{n}\right)= \begin{cases}\left(s_{1}, \ldots,+,-, \ldots, s_{n}\right), & \text { if } i \neq n,\left(s_{i}, s_{i+1}\right)=(-,+), \\
\left(s_{1}, \ldots, s_{n-2},+,+\right), & \text { if } i=n,\left(s_{n-1}, s_{n}\right)=(-,-), \\
0, & \text { otherwise, }\end{cases} \\
& \widetilde{f}_{i}\left(s_{1}, \ldots, s_{n}\right)= \begin{cases}\left(s_{1}, \ldots,-,+, \ldots, s_{n}\right), & \text { if } i \neq n,\left(s_{i}, s_{i+1}\right)=(+,-), \\
\left(s_{1}, \ldots, s_{n-2},-,-\right), & \text { if } i=n,\left(s_{n-1}, s_{n}\right)=(+,+), \\
0, & \text { otherwise. }\end{cases}
\end{aligned}
$$

The weight of each basis is

$$
\operatorname{wt}\left(\left(s_{1}, \ldots, s_{n}\right)\right)=\frac{1}{2}\left(s_{1} \epsilon_{1}+\cdots+s_{n} \epsilon_{n}\right) .
$$

Thus we can represent each element $\epsilon_{i}$ by half width tableau with entry $i$. We arrange the entries within a column according to the following order on the letters (smaller ones at higher places)

$$
1 \prec 2 \prec \cdots \prec n-1 \prec \frac{n}{n} \prec \overline{n-1} \prec \cdots \prec \overline{2} \prec \overline{1} \text {. }
$$

Here we do not introduce the order between $n$ and $\bar{n}$.

For the general case, we start by the highest weight element $u_{\lambda} \in B^{n, 2 s+1}$ where $\lambda=(2 s+$ 1) $\bar{\Lambda}_{n}$. Then represent $u_{\lambda}$ by the spin column $(+, \ldots,+)$ and $s$ columns of $1,2, \ldots, n$ such as

$$
u_{(2 s+1) \bar{\Lambda}_{n}}=\begin{array}{c|c|c|c|c|}
\hline 1 & 1 & 1 & \cdots & 1 \\
\hline 2 & 2 & 2 & \cdots & 2 \\
\hline \vdots & \vdots & \vdots & \ddots & \vdots \\
\hline n & n & n & \cdots & n \\
\hline
\end{array}
$$


In order to obtain the other tableaux, we embed $u_{\lambda}$ into $B^{r, 1} \otimes\left(B^{1,1}\right)^{\otimes r s}$ by using the Japanese reading word for the right $s$ columns and apply the Kashiwara operators $\widetilde{f}_{i}$ in all possible ways.

We can obtain the KN tableaux for the case $B^{n, 2 s}$ similarly if we ignore the leftmost spin column in $B^{n, 2 s+1}$ case.

KN tableaux for $\boldsymbol{B}^{\boldsymbol{n - 1 , 2 s + 1}}$. In the case $B^{n-1,1} \simeq B\left(\bar{\Lambda}_{n-1}\right)$, we can represent the basis as $\left(s_{1}, s_{2}, \ldots, s_{n}\right)$ where $s_{i}= \pm$ and $s_{1} s_{2} \cdots s_{n}=-1$. We can use the same formulae $(2.5)$ and (2.6) for the Kashiwara operators and (2.7) for the weight. For the general case $B^{n-1,2 s+1}$, we represent the highest weight element $u_{(2 s+1) \bar{\Lambda}_{n-1}} \in B^{n-1,2 s+1}$ by

$$
u_{(2 s+1) \bar{\Lambda}_{n-1}}=\begin{array}{c|c|c|c|c|c|}
\hline 1 & 1 & 1 & \cdots & 1 \\
\hline 2 & 2 & 2 & \cdots & 2 \\
\hline \vdots & \vdots & \vdots & \ddots & \vdots \\
\hline \bar{n} & \bar{n} & \bar{n} & \cdots & \bar{n} \\
\hline
\end{array}
$$

Here we obtain the tableau for $u_{(2 s+1)} \bar{\Lambda}_{n-1}$ by replacing all $n$ of the bottom row of $u_{(2 s+1)} \bar{\Lambda}_{n}$ by $\bar{n}$. Then we can use the Kashiwara operators to obtain all the tableaux of $B^{n-1,2 s+1}$. We can obtain tableaux for the case $B^{n-1,2 s}$ if we ignore the leftmost spin column.

\subsubsection{Filling map}

The purpose to introduce the KR tableaux is to give a rectangular presentation of elements of crystals as opposed to the KN tableaux whose shapes depend on the classical decompositions given in (2.3) and (2.4). The definition of the KR tableaux is given explicitly for the highest weight element of $B^{r, s}$ and extended to the general case by using the Kashiwara operators $\widetilde{f}_{i}$ as in the KN tableaux case.

To begin with we give a definition of the KR tableaux for the case $B^{r, s}(r \leq n-2)$. Let $u_{\lambda}$ be the $I_{0}$-highest element of $B^{r, s}$ with weight $\lambda=k_{r} \bar{\Lambda}_{r}+k_{r-2} \bar{\Lambda}_{r-2}+\cdots$. We denote by fill $\left(u_{\lambda}\right)$ the KR tableau representation of $u_{\lambda}$. Now we describe the algorithm to obtain fill $\left(u_{\lambda}\right)$ which we call the filling map. Let $k_{c}$ be the first odd integer in the sequence $k_{r-2}, k_{r-4}, \ldots$ If there is no such $k_{c}$, set $k_{c}=k_{-1}$, that is, $c=-1$. Let $t$ be the KN tableau representation of $u_{\lambda}$. We place $t$ at the top left corner of the $r$ by $s$ rectangle. In order to fill all the empty places, we start from the leftmost column of $t$ and move rightwards by the following procedure.

1. We do nothing for the height $r$ columns. If $c \geq 0$, remove one column from the height $c$ columns and move the columns that are shorter than $c$ to left by 1 .

2. For the columns whose heights are equal to or larger than $c$, put the following stuff to the empty places as much as possible. If the height of the corresponding columns is $h$, put the transpose of

\begin{tabular}{|c|c|c|c|}
\hline $\bar{r}$ & $\overline{r-1}$ & $\ldots$ & $\overline{h+1}$ \\
\hline$h+1$ & $h+2$ & $\ldots$ & $r$ \\
\hline
\end{tabular}

If $c=-1$, we stop here since all empty places are filled by this procedure.

3. For the remaining columns except for the rightmost one, put the following stuff recursively from left to right with recursively redefining an integer $x$. As the initial condition, set $x=c+1$.

If the corresponding column has height $h$, put the transpose of

\begin{tabular}{|l|l|l|l|l|l|l|l|}
\hline$y$ & $\ldots$ & $r-1$ & $r$ & $\bar{r}$ & $\ldots$ & $\overline{x+1}$ & $\bar{x}$ \\
\hline
\end{tabular}

to the empty place. Since the number of the empty places is $r-h$, we have $y=r-(x-h-2)$. We redefine $x=y$ and do the same procedure to the next column. 
4. By using the final output of $x$ from the last step, we put the transpose of the following to the rightmost column

\begin{tabular}{|l|l|l|l|l|l|l|l|}
\hline 1 & 2 & $\ldots$ & $y$ & $\bar{y}$ & $\ldots$ & $\overline{x+1}$ & $\bar{x}$ \\
\hline
\end{tabular}

Recall that the rightmost column is empty by Step 1 whenever $c \geq 0$. Then in order to achieve the above pattern, we have $y=(r+x-1) / 2$.

Example 2.3. Let us consider the $I_{0}$-highest element $u_{\lambda}$ of $B^{8,7}$ where $\lambda=\bar{\Lambda}_{8}+2 \bar{\Lambda}_{6}+\bar{\Lambda}_{4}+$ $2 \bar{\Lambda}_{2}$. In this case, we have $\left(k_{6}, k_{4}, k_{2}, k_{0}\right)=(2,1,2,1)$, thus $c=4$. We put the KN tableau representation of $u_{\lambda}$ into the 8 by 7 rectangle. Then

\begin{tabular}{|l|l|l|l|l|l|l|}
\hline 1 & 1 & 1 & 1 & 1 & 1 & \\
\hline 2 & 2 & 2 & 2 & 2 & 2 & \\
\hline 3 & 3 & 3 & 3 & & & \\
\hline 4 & 4 & 4 & 4 & & & \\
\hline 5 & 5 & 5 & & & & \\
\hline 6 & 6 & 6 & & & & \\
\hline 7 & & & & & & \\
\hline 8 & & & & & & \\
Step 1
\end{tabular}$\quad$\begin{tabular}{|l|l|l|l|l|l|l|l|}
\hline 1 & 1 & 1 & 1 & 1 & & \\
\hline 2 & 2 & 2 & 2 & 2 & & \\
\hline 3 & 3 & 3 & & & & \\
\hline 4 & 4 & 4 & & & & \\
\hline 5 & 5 & 5 & & & & \\
\hline 6 & 6 & 6 & & & & \\
\hline 7 & & & & & & \\
\hline 8 & & & & & & \\
\hline
\end{tabular}

Then we fill the empty places as

\begin{tabular}{|l|l|l|l|l|l|l|}
\hline & & & & & 5 & 1 \\
\hline & & & & & 6 & 2 \\
\hline & & & 7 & 5 & 7 & 3 \\
\hline & & & 8 & 6 & 8 & 4 \\
\hline & & & $\overline{8}$ & 7 & $\overline{8}$ & 5 \\
\hline & & & $\overline{7}$ & 8 & $\overline{7}$ & 6 \\
\hline & $\overline{8}$ & 7 & $\overline{6}$ & $\overline{8}$ & $\overline{6}$ & $\overline{6}$ \\
\hline & $\overline{7}$ & 8 & $\overline{5}$ & $\overline{7}$ & $\overline{5}$ & $\overline{5}$ \\
\hline
\end{tabular}

thus, fill $\left(u_{\lambda}\right)=$\begin{tabular}{|l|l|l|l|l|l|l|}
\hline 1 & 1 & 1 & 1 & 1 & 5 & 1 \\
\hline 2 & 2 & 2 & 2 & 2 & 6 & 2 \\
\hline 3 & 3 & 3 & 7 & 5 & 7 & 3 \\
\hline 4 & 4 & 4 & 8 & 6 & 8 & 4 \\
\hline 5 & 5 & 5 & $\overline{8}$ & 7 & $\overline{8}$ & 5 \\
\hline 6 & 6 & 6 & $\overline{7}$ & 8 & $\overline{7}$ & 6 \\
\hline 7 & $\overline{8}$ & 7 & $\overline{6}$ & $\overline{8}$ & $\overline{6}$ & $\overline{6}$ \\
\hline 8 & $\overline{7}$ & 8 & $\overline{5}$ & $\overline{7}$ & $\overline{5}$ & $\overline{5}$ \\
\hline
\end{tabular}

In order to define the KR tableaux for the spin cases, we start from the following expression for the highest weight elements (collection of $s$ spin columns)

$$
u_{s \bar{\Lambda}_{n-1}}=\begin{array}{|c|c|c|c|}
\hline 1 & 1 & \cdots & 1 \\
\hline 2 & 2 & \cdots & 2 \\
\hline \vdots & \vdots & \ddots & \vdots \\
\hline \bar{n} & \bar{n} & \cdots & \bar{n} \\
\hline
\end{array}, \quad u_{s \bar{\Lambda}_{n}}=\begin{array}{|c|c|c|c|c|}
\hline 1 & 1 & \cdots & 1 \\
\hline 2 & 2 & \cdots & 2 \\
\hline \vdots & \vdots & \ddots & \vdots \\
\hline n & n & \cdots & n \\
\hline
\end{array}
$$

and apply the Kashiwara operators $\tilde{f}_{i}\left(i \in I_{0}\right)$ for all possible ways to obtain the rest of the elements of $B^{n-1, s}$ and $B^{n, s}$.

From Remark 5.2 and Remark 5.4 in [24], it follows that the new Kirillov-Reshetikhin (KR) tableaux carry a crystal structure obtained by their reading word which gives a natural embedding of $B(\lambda)$ into $B\left(\Lambda_{1}\right)^{\otimes r s}$. Since each classical factor in $B^{r, s}$ occurs with multiplicity 1 , we can use the Kirillov-Reshetikhin tableaux representation in place of the usual Kashiwara-Nakashima tableaux representation.

For the later purposes, we introduce several operations on the KR tableaux representation of crystals. 
Definition 2.4. We use the KR tableaux representation for the element $b=b_{L} \otimes b_{L-1} \otimes \cdots \otimes b_{1} \in$ $B^{r_{L}, s_{L}} \otimes B^{r_{L-1}, s_{L-1}} \otimes \cdots \otimes B^{r_{1}, s_{1}}$. Then we define the following operations.

(1) Suppose that $B^{r_{L}, s_{L}}=B^{1,1}$. Then we define the operation called left-hat by

$$
\operatorname{lh}(b)=b_{L-1} \otimes \cdots \otimes b_{1} \in B^{r_{L-1}, s_{L-1}} \otimes \cdots \otimes B^{r_{1}, s_{1}} .
$$

(2) Suppose that $B^{r_{L}, s_{L}}=B^{r_{L}, 1}$ and $r_{L}>1$. Thus $b_{L}$ has the form $b_{L}=$\begin{tabular}{c|c|}
\hline$t_{1}$ \\
\hline$\vdots$ \\
\hline$t_{r_{L}-1}$ \\
\hline$t_{r_{L}}$ \\
\hline
\end{tabular} Then we define the operation called left-box by

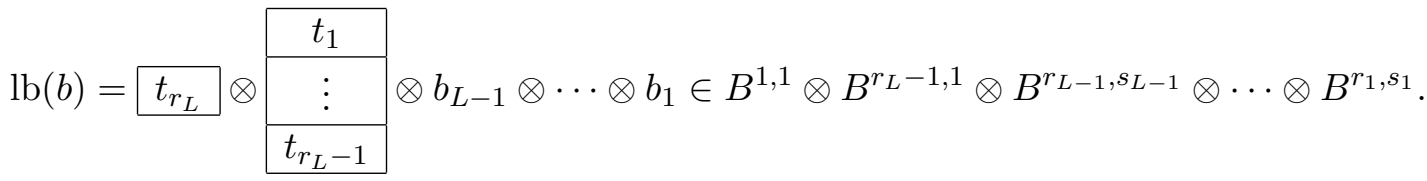

(3) Suppose that $s_{L}>1$. Let $c_{i}$ be the $i$ th column of $b_{L}$ such that $b_{L}=c_{1} c_{2} \cdots c_{s_{L}}$. Then we define the operation called left-split by

$$
\operatorname{ls}(b)=c_{1} \otimes c_{2} \cdots c_{s_{L}} \otimes b_{L-1} \otimes \cdots \otimes b_{1} \in B^{r_{L}, 1} \otimes B^{r_{L}, s_{L}-1} \otimes B^{r_{L-1}, s_{L-1}} \otimes \cdots \otimes B^{r_{1}, s_{1}} .
$$

\section{Rigged configurations and the main bijection}

\subsection{Definition of the rigged configurations}

Our strategy to define the rigged configurations of type $\mathfrak{g}=D_{n}^{(1)}$ has three steps. First we define the highest weight rigged configurations. Next we define the classical Kashiwara operators $\widetilde{e}_{i}$ and $\widetilde{f}_{i}$ for $i \in I_{0}$ on the rigged configurations. Finally, we define the general rigged configurations by all possible applications of the operators $\widetilde{f}_{i}$ on highest weight rigged configurations.

Let us prepare several notation to describe the rigged configurations. The rigged configurations are combinatorial objects made up with partitions $\nu^{(a)}$ and integers $J^{(a)}$ for $a \in I_{0}$. $\nu^{(a)}=$ $\left(\nu_{1}^{(a)}, \ldots, \nu_{k}^{(a)}\right)$ is a partition called the configuration and $J^{(a)}=\left(J_{1}^{(a)}, \ldots, J_{k}^{(a)}\right)$ is a sequence of integers called the rigging which are associated with rows of the corresponding configuration. We should regard that the pairs $\left(\nu^{(a)}, J^{(a)}\right)$ are located on the vertices $I_{0}$ of the Dynkin diagram of $D_{n}^{(1)}$. We denote by $m_{l}^{(a)}(\nu)$ the number of length $l$ rows of $\nu^{(a)}$. We will abbreviate the pair $\left(\nu^{(a)}, J^{(a)}\right)$ by $(\nu, J)^{(a)}$ and call the components of $(\nu, J)^{(a)}=\left\{\left(\nu_{1}^{(a)}, J_{1}^{(a)}\right), \ldots,\left(\nu_{k}^{(a)}, J_{k}^{(a)}\right)\right\}$ the strings.

The rigged configurations also depend on the shape of the tensor product $B=B^{r_{L}, s_{L}} \otimes$ $\cdots \otimes B^{r_{1}, s_{1}}$. Let $L_{l}^{(a)}$ be the number of the component $B^{a, l}$ within $B$. Then we define the partition $\mu^{(a)}$ such that the number of length $l$ rows of $\mu^{(a)}$ is equal to $L_{l}^{(a)}$. Thus the rigged configuration $(\nu, J)$ is described by the sequence of configurations and the riggings

$$
(\nu, J)=\left((\nu, J)^{(1)}, \ldots,(\nu, J)^{(n-1)},(\nu, J)^{(n)}\right)
$$

together with additional data $\mu=\left(\mu^{(1)}, \ldots, \mu^{(n-1)}, \mu^{(n)}\right)$ under certain constraints to be described below. We denote by $Q_{l}^{(a)}(\nu)$ (resp. $Q_{l}^{(a)}(\mu)$ ) the number of boxes in the first $l$ columns of $\nu^{(a)}\left(\right.$ resp. $\left.\mu^{(a)}\right)$;

$$
Q_{l}^{(a)}(\nu)=\sum_{j>0} \min \{l, j\} m_{j}^{(a)}(\nu), \quad Q_{l}^{(a)}(\mu)=\sum_{j>0} \min \{l, j\} L_{j}^{(a)} .
$$


Therefore we have $Q_{\infty}^{(a)}(\nu)=\left|\nu^{(a)}\right|$ and $Q_{\infty}^{(a)}(\mu)=\left|\mu^{(a)}\right|$ where $\left|\nu^{(a)}\right|$ and $\left|\mu^{(a)}\right|$ are the total number of boxes in $\nu^{(a)}$ and $\mu^{(a)}$, respectively. From the data $\mu$ and $\nu$ we define the vacancy number $P_{l}^{(a)}(\mu, \nu)$ (hereafter we will abbreviate as $\left.P_{l}^{(a)}(\nu)\right)$ by the formula

$$
\begin{aligned}
P_{l}^{(a)}(\nu) & =Q_{l}^{(a)}(\mu)+\sum_{b \in I_{0}} A_{a b} Q_{l}^{(b)}(\nu) \\
& =Q_{l}^{(a)}(\mu)-2 Q_{l}^{(a)}(\nu)+\sum_{\substack{b \in I_{0} \\
b \sim a}} Q_{l}^{(b)}(\nu),
\end{aligned}
$$

where $A_{a b}$ is the Cartan matrix of $\mathfrak{g}_{0}=D_{n}$ and $a \sim b$ means that the vertices $a$ and $b$ are connected by a single edge on the Dynkin diagram.

Definition 3.1. The data $\mu$ and $(\nu, J)$ is the highest weight rigged configuration if all the strings $\left(\nu_{i}^{(a)}, J_{i}^{(a)}\right)$ and the corresponding vacancy numbers satisfy the following condition

$$
0 \leq J_{i}^{(a)} \leq P_{\nu_{i}^{(a)}}^{(a)}(\nu)
$$

Remark 3.2. If $\nu^{(n-1)}=\nu^{(n)}=\varnothing$, then we can identify the rigged configuration as type $A_{n-2}^{(1)}$. In this way we can recover proofs for type $A_{n}^{(1)}$ from those for type $D_{n}^{(1)}$.

Example 3.3. The following object is a highest weight rigged configuration corresponding to the tensor product $B^{3,2} \otimes B^{3,1} \otimes B^{2,2} \otimes B^{1,2} \otimes B^{1,1}$ of type $D_{5}^{(1)}$ :

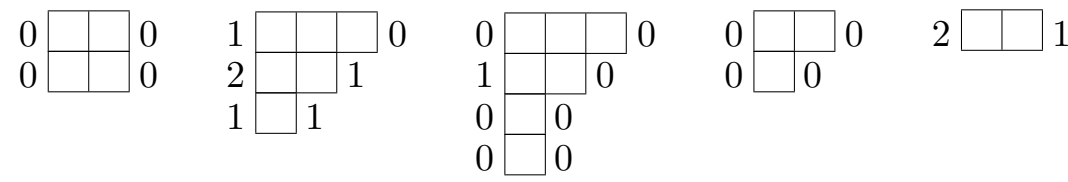

Here we put the vacancy number (resp. rigging) on the left (resp. right) of the corresponding row of the configuration represented by a Young diagram. By the rigged configuration bijection $\Phi$ (see Section 3.3) the above rigged configuration $(\nu, J)$ corresponds to the following highest weight tensor product:

$$
\Phi(\nu, J)=\begin{array}{|l|l|}
\hline 1 & 3 \\
\hline 2 & \overline{5} \\
\hline 4 & 5 \\
\hline
\end{array} \otimes \begin{array}{|l|l|l|l|}
\hline 1 \\
\hline 4 \\
\hline 5
\end{array} \otimes \begin{array}{|l|l|l|}
\hline 2 & 1 \\
\hline 3 & \overline{1} \\
\hline
\end{array} \otimes \otimes \begin{array}{ll}
1 & 2 \\
\hline
\end{array}
$$

The weight $\lambda$ of the rigged configuration is defined by the relation (sometimes called the $(L, \lambda)$-configuration condition)

$$
\sum_{a \in I_{0}, i>0} i m_{i}^{(a)} \alpha_{a}=\sum_{a \in I_{0}, i>0} i L_{i}^{(a)} \bar{\Lambda}_{a}-\lambda .
$$

If we expand $\lambda$ by the basis $\epsilon_{i}$, we can rewrite as

$$
\lambda=\sum_{i} \lambda_{i} \epsilon_{i}=\sum_{a \in I_{0}}\left(\left|\mu^{(a)}\right| \bar{\Lambda}_{a}-\left|\nu^{(a)}\right| \alpha_{a}\right) .
$$

Then we can use the expressions (2.1) and (2.2) to obtain the explicit expressions for the weight $\lambda_{i}$. We write the weight of the rigged configuration by $\operatorname{wt}(\nu, J)$.

Following [32] we introduce the classical Kashiwara operators on the rigged configurations and use them to define the general rigged configurations. For the string $(l, x)$ of $(\nu, J)^{(i)}$, we call the quantity $P_{l}^{(i)}(\nu)-x$ the corigging. 
Definition 3.4. The rigged configurations are obtained by all possible applications of the Kashiwara operators $\widetilde{f}_{i}\left(i \in I_{0}\right)$ on the highest weight rigged configurations. Here the Kashiwara operators on the rigged configurations are defined as follows. Let $x_{\ell}$ be the smallest rigging of $(\nu, J)^{(i)}$.

(1) Let $\ell$ be the minimal length of the strings of $(\nu, J)^{(i)}$ with the rigging $x_{\ell}$. If $x_{\ell} \geq 0$, define $\widetilde{e}_{i}(\nu, J)=0$. Otherwise $\widetilde{e}_{i}(\nu, J)$ is obtained by replacing the string $\left(\ell, x_{\ell}\right)$ by $\left(\ell-1, x_{\ell}+1\right)$ while changing all other riggings to keep coriggings fixed.

(2) Let $\ell$ be the maximal length of the strings of $(\nu, J)^{(i)}$ with the rigging $x_{\ell}$. $\tilde{f}_{i}(\nu, J)$ is obtained by the following procedure. If $x_{\ell}>0$, add a string $(1,-1)$ to $(\nu, J)^{(i)}$. Otherwise replace the string $\left(\ell, x_{\ell}\right)$ by $\left(\ell+1, x_{\ell}-1\right)$. Change other riggings to keep coriggings fixed. If the new rigging is strictly larger than the corresponding new vacancy number, define $\widetilde{f}_{i}(\nu, J)=0$.

By definition every rigging is always less than or equal to the corresponding vacancy number.

Proposition 3.5. The above defined Kashiwara operators satisfy the following relations

$$
\operatorname{wt}\left(\widetilde{e}_{i}(\nu, J)\right)=\operatorname{wt}(\nu, J)+\alpha_{i}, \quad \operatorname{wt}\left(\widetilde{f}_{i}(\nu, J)\right)=\operatorname{wt}(\nu, J)-\alpha_{i} .
$$

Moreover $\widetilde{f}_{i}(\nu, J) \neq 0 \Longrightarrow \widetilde{e}_{i} \widetilde{f}_{i}(\nu, J)=(\nu, J)$ and $\widetilde{e}_{i}(\nu, J) \neq 0 \Longrightarrow \widetilde{f}_{i} \widetilde{e}_{i}(\nu, J)=(\nu, J)$.

Proof. The relations for wt follow from (3.2). In order to show $\widetilde{e}_{i} \widetilde{f}_{i}(\nu, J)=(\nu, J)$, suppose that $\widetilde{f}_{i}$ creates the string $\left(\ell+1, x_{\ell}-1\right)$. Let $(\widetilde{\nu}, \widetilde{J})=\widetilde{f}_{i}(\nu, J)$. Suppose that $0<\ell$.

(a) Let $\left(k, x_{k}\right)$ be a string of $(\nu, J)^{(i)}$ which satisfies $k \leq \ell$. Since $\widetilde{f}_{i}$ acts on the string with smallest rigging, we have $x_{k} \geq x_{\ell}$. Recall that we have $P_{k}^{(i)}(\widetilde{\nu})=P_{k}^{(i)}(\nu)$ since $\widetilde{f}_{i}$ adds a box to the $(\ell+1)$-th column. Thus the string $\left(k, x_{k}\right)$ remains as it is after $\tilde{f}_{i}$ and its rigging satisfies $x_{k}>x_{\ell}-1$.

(b) Let $\left(k, x_{k}\right)$ be a string of $(\nu, J)^{(i)}$ which satisfies $\ell<k$. Since $\widetilde{f}_{i}$ acts on the longest string with rigging $x_{\ell}$, we have $x_{\ell}<x_{k}$. Recall that we have $P_{k}^{(i)}(\widetilde{\nu})=P_{k}^{(i)}(\nu)-2$ in this case. Thus the string $\left(k, x_{k}\right)$ becomes $\left(k, x_{k}-2\right)$ after $\tilde{f}_{i}$. Then its rigging satisfies $x_{k}-2 \geq x_{\ell}-1$.

Thus the string $\left(\ell+1, x_{\ell}-1\right)$ is the shortest string among the strings of the smallest rigging of $(\widetilde{\nu}, \widetilde{J})^{(i)}$, hence $\widetilde{e}_{i}$ will act on it. Therefore we obtain $\widetilde{e}_{i} \widetilde{f}_{i}(\nu, J)=(\nu, J)$. Let us consider the case $\ell=0$. Then we can use the same arguments as before if we put $\left(\ell, x_{\ell}\right)=(0,0)$.

The opposite relation $\widetilde{f}_{i} \widetilde{e}_{i}(\nu, J)=(\nu, J)$ can be shown similarly.

Finally, let us define the maps $\varepsilon_{i}, \varphi_{i}:(\nu, J) \rightarrow \mathbb{Z}$ by

$$
\varepsilon_{i}(\nu, J)=\max \left\{m \geq 0 \mid \widetilde{e}_{i}^{m}(\nu, J) \neq 0\right\}, \quad \varphi_{i}(\nu, J)=\max \left\{m \geq 0 \mid \widetilde{f}_{i}^{m}(\nu, J) \neq 0\right\} .
$$

\subsection{Convexity relations of the vacancy numbers and their applications}

In this subsection, we introduce a fundamental property of the vacancy numbers called the convexity relation. As an application, we provide a direct proof of the fact that the crystal structure on the set of the rigged configurations indeed satisfies the axiom of the $U_{q}\left(\mathfrak{g}_{0}\right)$-crystals. Finally, we prepare refined estimates for the vacancy numbers which are necessary in the later arguments.

One of the basic properties of the vacancy number is the following convexity relation.

Proposition 3.6 (convexity). Suppose that $m_{l}^{(a)}(\nu)=0$ for some $l \geq 1$. Then the vacancy numbers satisfy the following convexity relation

$$
2 P_{l}^{(a)}(\nu) \geq P_{l-1}^{(a)}(\nu)+P_{l+1}^{(a)}(\nu) .
$$


Proof. Recall that the functions $Q_{k}^{(a)}(\mu)$ and $Q_{k}^{(b)}(\nu)$ for $b \neq a$ are all upper convex functions of $k$. On the other hand, since $m_{l}^{(a)}(\nu)=0$, the function $Q_{k}^{(a)}(\nu)$ is a linear function between $l-1 \leq k \leq l+1$. Thus the combination in (3.1) gives the upper convex relation for the vacancy numbers.

By repeated use of the above convexity relation, we obtain several useful criteria.

Corollary 3.7. Suppose that $m_{k}^{(a)}(\nu)=0$ for all $l_{1}<k<l_{2}$.

(1) $P_{k}^{(a)}(\nu) \geq \min \left\{P_{l_{1}}^{(a)}(\nu), P_{l_{2}}^{(a)}(\nu)\right\}$.

(2) For some $k$ satisfying $l_{1}<k<l_{2}$ suppose that we have $P_{l_{1}}^{(a)}(\nu) \geq P_{k}^{(a)}(\nu)<P_{l_{2}}^{(a)}(\nu)$ or $P_{l_{1}}^{(a)}(\nu)>P_{k}^{(a)}(\nu) \leq P_{l_{2}}^{(a)}(\nu)$. Then the corresponding rigged configuration is forbidden.

(3) For some $k$ satisfying $l_{1}<k<l_{2}$ suppose that we have $P_{l_{1}}^{(a)}(\nu) \geq P_{k}^{(a)}(\nu) \leq P_{l_{2}}^{(a)}(\nu)$. Then the only admissible situation is $P_{l_{1}}^{(a)}(\nu)=P_{l_{1}+1}^{(a)}(\nu)=\cdots=P_{l_{2}}^{(a)}(\nu)$.

Since we will use these properties so many times in the rest of the paper, we will sometimes use these relations without giving an explicit reference.

Let us give the first application of the convexity relations.

Theorem 3.8. Let $x_{\ell}$ be the smallest rigging of $(\nu, J)^{(i)}$ and define $s=\min \left\{0, x_{\ell}\right\}$. If $\nu^{(i)}=\varnothing$, set $s=0$. Then we have

(1) $\varepsilon_{i}(\nu, J)=-s$,

(2) $\varphi_{i}(\nu, J)=P_{\infty}^{(i)}(\nu)-s$.

Proof. (1) We proceed by induction on $-s$. Let $(\widetilde{\nu}, \widetilde{J}):=\widetilde{e}_{i}(\nu, J), \widetilde{x}_{\widetilde{\ell}}$ be the smallest rigging of $(\widetilde{\nu}, \widetilde{J})^{(i)}$ and define $\widetilde{s}=\min \left\{0, \widetilde{x}_{\widetilde{\ell}}\right\}$.

Suppose that $-s=0$. This implies that $x_{\ell} \geq 0$. Then by the definition of $\widetilde{e}_{i}$ we have $\widetilde{e}_{i}(\nu, J)=0$ as requested. Suppose that $-s>0$. Then we have $s=x_{\ell}<0$. Suppose that $\widetilde{e}_{i}$ acts on the string $\left(\ell, x_{\ell}\right)$. Let us analyze the behaviors of the riggings by $\widetilde{e}_{i}$.

(a) The string $\left(\ell, x_{\ell}\right)$ becomes $\left(\ell-1, x_{\ell}+1\right)$.

(b) Let $\left(k, x_{k}\right)$ be an arbitrary string of $(\nu, J)^{(i)}$ satisfying $k<\ell$. Since $\widetilde{e}_{i}$ acts on the shortest string with rigging $x_{\ell}$, we have $x_{k}>x_{\ell}$. From $k<\ell$, we see that $\widetilde{e}_{i}$ will not change corigging of $\left(k, x_{k}\right)$, thus the string $\left(k, x_{k}\right)$ remains as it is in $(\widetilde{\nu}, \widetilde{J})$.

(c) Let $\left(k, x_{k}\right)$ be an arbitrary string of $(\nu, J)^{(i)}$ satisfying $\ell \leq k$. Since $x_{\ell}$ is the minimal rigging of $(\nu, J)^{(i)}$, we have $x_{\ell} \leq x_{k}$. Recall that we have $P_{k}^{(i)}(\widetilde{\nu})=P_{k}^{(i)}(\nu)+2$ since $\widetilde{e}_{i}$ removes a box from $\ell$ th column of $\nu^{(i)}$. Thus $\widetilde{e}_{i}$ makes the string $\left(k, x_{k}\right)$ into $\left(k, x_{k}+2\right)$, in particular, its rigging satisfies $x_{\ell}+1<x_{k}+2$.

To summarize, we have $\widetilde{x}_{\widetilde{\ell}}=x_{\ell}+1$ and thus $\widetilde{s}=s+1$, that is, $-\widetilde{s}=\varepsilon_{i}(\nu, J)-1=\varepsilon_{i}(\widetilde{\nu}, \widetilde{J})$ as requested.

(2) This is proved in [32, Lemma 3.6]. However, for the sake of the completeness, we include a proof here. We prove by induction on $P_{\infty}^{(i)}(\nu)-s$. Suppose that $\tilde{f}_{i}$ acting on $(\nu, J)$ creates a length $\ell+1$ string. By definition of $s, \widetilde{f}_{i}$ creates the string $(\ell+1, s-1)$ of $(\widetilde{\nu}, \widetilde{J}):=\widetilde{f}_{i}(\nu, J)$. Let $\widetilde{x}_{\widetilde{\ell}}$ be the smallest rigging of $(\widetilde{\nu}, \widetilde{J})^{(i)}$ and define $\widetilde{s}=\min \left\{0, \widetilde{x}_{\tilde{\ell}}\right\}$.

Step 1. Let us consider the case $P_{\infty}^{(i)}(\nu)-s=0$. Suppose that $x_{\ell} \leq 0$. Then we have $\ell>0$ and $s=x_{\ell}$, thus, $P_{\infty}^{(i)}(\nu)=x_{\ell}$. Let $j$ be the maximal integer such that $\ell \leq j$ and $m_{j}^{(i)}(\nu)>0$. Suppose if possible that $\ell<j$. Let us consider the string $\left(j, x_{j}\right)$. Since $\widetilde{f}_{i}$ acts on the longest 
string with rigging $x_{\ell}$, the assumption $\ell<j$ implies that $x_{\ell}<x_{j}$. Note that we have $x_{j} \leq P_{j}^{(i)}(\nu)$ since $(\nu, J)$ is the rigged configuration. Thus $x_{\ell}<P_{j}^{(i)}(\nu)$. Recall that the definition (3.1) implies that there exists a large integer $L$ such that $P_{L}^{(i)}(\nu)=P_{L+1}^{(i)}(\nu)=\cdots=P_{\infty}^{(i)}(\nu)=x_{\ell}$. Since $j$ is the maximal integer such that $m_{j}^{(i)}(\nu)>0$, we see that $P_{k}^{(i)}(\nu)$ satisfy the convexity relation between $j \leq k \leq \infty$. In particular, the relation $P_{j}^{(i)}(\nu)>P_{L}^{(i)}(\nu)=P_{L+1}^{(i)}(\nu)$ for $j<L<L+1$ is a contradiction. Therefore we have $\ell=j$ and thus there is no string of $(\nu, J)^{(i)}$ that is longer than $\ell$. Then from the convexity relation of $P_{k}^{(i)}(\nu)$ between $\ell \leq k \leq \infty$, the only possibility that is compatible with the requirement $P_{\ell}^{(i)}(\nu) \geq x_{\ell}$ is the relation $P_{\ell}^{(i)}(\nu)=$ $P_{\ell+1}^{(i)}(\nu)=\cdots=x_{\ell}$.

Let us consider the string $\left(\ell+1, x_{\ell}-1\right)$ of $(\widetilde{\nu}, \widetilde{J})^{(i)}$ created by $\widetilde{f}_{i}$. Due to the extra box added by $\widetilde{f}_{i}$, we have $P_{\ell+1}^{(i)}(\widetilde{\nu})=P_{\ell+1}^{(i)}(\nu)-2=x_{\ell}-2$. Thus the string $\left(\ell+1, x_{\ell}-1\right)$ of $(\widetilde{\nu}, \widetilde{J})^{(i)}$ has the rigging that is strictly larger than the corresponding vacancy number. Thus we have $\widetilde{f}_{i}(\nu, J)=0$ as requested.

Finally let us consider the case $x_{\ell}>0$. Then we have $P_{\infty}^{(i)}(\nu)=0$ by $s=0$. Let $j$ be the maximal integer such that $m_{j}^{(i)}(\nu)>0$ and consider the corresponding string $\left(j, x_{j}\right)$. Since $x_{\ell}$ is the minimal rigging, we have $P_{j}^{(i)}(\nu) \geq x_{j} \geq x_{\ell}>0$. Then for a sufficiently large integer $L$, we have $P_{j}^{(i)}(\nu)>P_{L}^{(i)}(\nu)=P_{L+1}^{(i)}(\nu)=\cdots=0$. This is a contradiction since $P_{k}^{(i)}(\nu)$ must satisfy the convexity relation between $j \leq k \leq \infty$. Therefore we see that there is no string in $(\nu, J)^{(i)}$. This is a contradiction since we assume that $x_{\ell}>0$. Hence this case cannot happen.

Step 2. Let us consider the case $P_{\infty}^{(i)}(\nu)-s>0$. Suppose that $x_{\ell} \leq 0$. Then we have $P_{\infty}^{(i)}(\nu)>s=x_{\ell}$. Let $j$ be the minimal integer such that $\ell<j$ and $m_{j}^{(i)}(\nu)>0$. If there is no such $j$, set $j=\infty$. Then we have $x_{\ell}<P_{j}^{(i)}(\nu)$. For, if $j<\infty$ we have $x_{\ell}<x_{j} \leq P_{j}^{(i)}(\nu)$ as in the previous step and if $j=\infty$ we have $x_{\ell}<P_{j}^{(i)}(\nu)$ by the assumption.

Let us show that

$$
x_{\ell}<P_{\ell+1}^{(i)}(\nu)
$$

If $j=\ell+1$ this relation is already confirmed. Thus suppose that $\ell+1<j$. Suppose if possible that we have $x_{\ell} \geq P_{\ell+1}^{(i)}(\nu)$. Then we have $P_{\ell}^{(i)}(\nu) \geq P_{\ell+1}^{(i)}(\nu)<P_{j}^{(i)}(\nu)$ by $x_{\ell} \leq P_{\ell}^{(i)}(\nu)$ and $x_{\ell}<P_{j}^{(i)}(\nu)$. This is a contradiction since $P_{k}^{(i)}(\nu)$ must satisfy the convexity relation between $\ell \leq k \leq j$. In conclusion, we have $x_{\ell}<P_{\ell+1}^{(i)}(\nu)$.

Since $\widetilde{f}_{i}$ adds a box to the $(\ell+1)$-th column of $\nu^{(i)}$, we have $P_{\ell+1}^{(i)}(\widetilde{\nu})=P_{\ell+1}^{(i)}(\nu)-2 \geq x_{\ell}-1$ by (3.3). Therefore the string $\left(\ell+1, x_{\ell}-1\right)$ of $(\widetilde{\nu}, \widetilde{J})^{(i)}$ created by $\widetilde{f}_{i}$ has the rigging which is smaller than or equal to the corresponding vacancy number. Hence we have $\widetilde{f}_{i}(\nu, J) \neq 0$.

Let us determine $\widetilde{s}$.

(a) $\tilde{f}_{i}$ makes the string $\left(\ell+1, x_{\ell}-1\right)$. Below we consider the remaining strings of $(\nu, J)^{(i)}$.

(b) Let $\left(k, x_{k}\right)$ be an arbitrary string of $(\nu, J)^{(i)}$ satisfying $\ell<k$. Since $\tilde{f}_{i}$ acts on the longest string with rigging $x_{\ell}, \ell<k$ implies that $x_{\ell}<x_{k}$. Recall that we have $P_{k}^{(i)}(\widetilde{\nu})=P_{k}^{(i)}(\nu)-2$ since $\widetilde{f}_{i}$ adds a box to the $(\ell+1)$ th column of $\nu^{(i)}$. In order to keep the corigging, the string $\left(k, x_{k}\right)$ becomes $\left(k, x_{k}-2\right)$ after $\widetilde{f}_{i}$. In particular, the new rigging satisfies $x_{\ell}-1 \leq x_{k}-2$.

(c) Let $\left(k, x_{k}\right)$ be an arbitrary string of $(\nu, J)^{(i)}$ satisfying $k \leq \ell$. Then we have $x_{k} \geq x_{\ell}$. Since $\widetilde{f}_{i}$ does not change the corigging of the string, the string $\left(k, x_{k}\right)$ remains as it is after $\widetilde{f}_{i}$. 
To summarize, we have $\widetilde{s}=x_{\ell}-1=s-1$. Since we have $P_{\infty}^{(i)}(\widetilde{\nu})=P_{\infty}^{(i)}(\nu)-2$, we have $P_{\infty}^{(i)}(\widetilde{\nu})-\widetilde{s}=P_{\infty}^{(i)}(\nu)-s-1=\varphi_{i}(\nu, J)-1=\varphi_{i}(\widetilde{\nu}, \widetilde{J})$ as requested.

Finally consider the case $x_{\ell}>0$. In this case we have $s=0$. Then $\tilde{f}_{i}$ adds the string $(1,-1)$ to $(\nu, J)^{(i)}$. If we show that $\widetilde{f}_{i}(\nu, J) \neq 0$, then we have $\widetilde{s}=-1=s-1$ as requested. This can be done if we formally setting $\left(\ell, x_{\ell}\right)=(0,0)$ in the proof of $(3.3)$ to deduce that $P_{1}^{(i)}(\widetilde{\nu})=P_{1}^{(i)}(\nu)-2 \geq-1$.

Corollary 3.9. Let $\lambda=\operatorname{wt}(\nu, J)$. Then we have

$$
\left\langle h_{i}, \lambda\right\rangle=\varphi_{i}(\nu, J)-\varepsilon_{i}(\nu, J) .
$$

Proof. From Theorem 3.8, we have $\varphi_{i}(\nu, J)-\varepsilon_{i}(\nu, J)=\left(P_{\infty}^{(i)}(\nu)-s\right)-(-s)=P_{\infty}^{(i)}(\nu)$. On the other hand, from (3.2) we have (see also definitions in Section 2.1)

$$
\begin{aligned}
\left\langle h_{i}, \lambda\right\rangle & =\sum_{a \in I_{0}}\left(\left|\mu^{(a)}\right|\left\langle h_{i}, \bar{\Lambda}_{a}\right\rangle-\left|\nu^{(a)}\right|\left\langle h_{i}, \alpha_{a}\right\rangle\right) \\
& =\left|\mu^{(i)}\right|-2\left|\nu^{(i)}\right|+\sum_{a \in I_{0}, a \sim i}\left|\nu^{(a)}\right|=P_{\infty}^{(i)}(\nu) .
\end{aligned}
$$

Hence we have $\left\langle h_{i}, \lambda\right\rangle=\varphi_{i}(\nu, J)-\varepsilon_{i}(\nu, J)$.

Example 3.10. Let us consider the following element of $\left(B^{1,1}\right)^{\otimes 8}$

$$
b=1 \otimes 2 \otimes 3 \otimes 1 \otimes 1 \otimes 2 \otimes 1 \otimes 1
$$

We can see that $\varphi_{1}(b)=3$. Then we can apply $\tilde{f}_{1}$ as follows

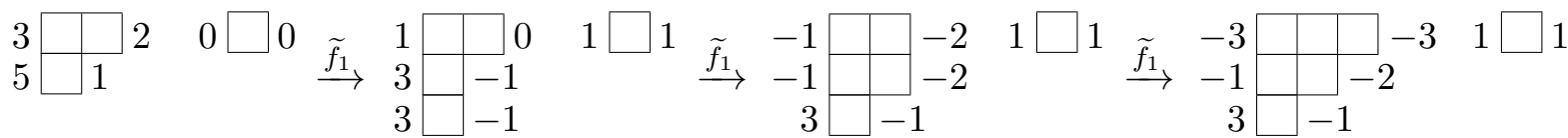

The leftmost rigged configuration corresponds to the path $b$. For $(\nu, J)=\Phi^{-1}(b)$ we have $P_{\infty}^{(1)}(\nu)=3$ and $s$ in Theorem 3.8 is $s=0$ since the smallest rigging is 1 . Therefore we have $\varphi_{1}(\nu, J)=P_{\infty}^{(1)}(\nu)-s=3$.

Combining Proposition 3.5 and Corollary 3.9, we obtain the following result.

Theorem 3.11. The maps $\varepsilon_{i}, \varphi_{i}$, wt and the Kashiwara operators $\widetilde{e}_{i}, \widetilde{f}_{i}$ defined for the rigged configurations satisfy the axioms for the $U_{q}\left(\mathfrak{g}_{0}\right)$-crystals for $\mathfrak{g}=A_{n}^{(1)}$ or $D_{n}^{(1)}$ given in Definition 2.1.

For the later purposes, let us prepare some refined estimates related with the convexity relations. The first one is an intuitive estimate.

Lemma 3.12. Let $m_{l}^{(i)}(\nu)=m$. Then $P_{l-1}^{(i)}(\nu), P_{l}^{(i)}(\nu), P_{l+1}^{(i)}(\nu)-2 m$ satisfy the convexity relation.

Proof. Let $\left(\nu^{\prime}, J\right)$ be the fictitious rigged configuration obtained by replacing all length $l$ strings of $(\nu, J)^{(i)}$ by length $l+1$ strings. From the similar argument of the proof of Proposition 3.6, we can show that $P_{l-1}^{(i)}\left(\nu^{\prime}\right), P_{l}^{(i)}\left(\nu^{\prime}\right), P_{l+1}^{(i)}\left(\nu^{\prime}\right)$ satisfy the convexity relation. Since $P_{l-1}^{(i)}\left(\nu^{\prime}\right)=$ $P_{l-1}^{(i)}(\nu), P_{l}^{(i)}\left(\nu^{\prime}\right)=P_{l}^{(i)}(\nu)$ and $P_{l+1}^{(i)}\left(\nu^{\prime}\right)=P_{l+1}^{(i)}(\nu)-2 m$ we obtain the assertion.

For some purposes, we need a more refined estimate as follows. 
Lemma 3.13. Suppose that $l \geq 1$. For $1 \leq a<n-2$ we have

$$
-P_{l-1}^{(a)}(\nu)+2 P_{l}^{(a)}(\nu)-P_{l+1}^{(a)}(\nu) \geq m_{l}^{(a-1)}(\nu)-2 m_{l}^{(a)}(\nu)+m_{l}^{(a+1)}(\nu),
$$

and for $n-2 \leq a$ we have

$$
\begin{aligned}
& -P_{l-1}^{(n-2)}(\nu)+2 P_{l}^{(n-2)}(\nu)-P_{l+1}^{(n-2)}(\nu) \geq m_{l}^{(n-3)}(\nu)-2 m_{l}^{(n-2)}(\nu)+m_{l}^{(n-1)}(\nu)+m_{l}^{(n)}(\nu), \\
& -P_{l-1}^{(n-1)}(\nu)+2 P_{l}^{(n-1)}(\nu)-P_{l+1}^{(n-1)}(\nu) \geq m_{l}^{(n-2)}(\nu)-2 m_{l}^{(n-1)}(\nu), \\
& -P_{l-1}^{(n)}(\nu)+2 P_{l}^{(n)}(\nu)-P_{l+1}^{(n)}(\nu) \geq m_{l}^{(n-2)}(\nu)-2 m_{l}^{(n)}(\nu) .
\end{aligned}
$$

Proof. Since $Q_{l}^{(a)}(\nu)$ counts the number of boxes of the left $l$ columns of $\nu$, we have

$$
\begin{aligned}
& -Q_{l-1}^{(a)}(\nu)+2 Q_{l}^{(a)}(\nu)-Q_{l+1}^{(a)}(\nu)=\left\{Q_{l}^{(a)}(\nu)-Q_{l-1}^{(a)}(\nu)\right\}-\left\{Q_{l+1}^{(a)}(\nu)-Q_{l}^{(a)}(\nu)\right\} \\
& \quad=\sum_{k \geq l} m_{k}^{(a)}(\nu)-\sum_{k \geq l+1} m_{k}^{(a)}(\nu)=m_{l}^{(a)}(\nu) .
\end{aligned}
$$

Similarly, we obtain

$$
-Q_{l-1}^{(a)}(\mu)+2 Q_{l}^{(a)}(\mu)-Q_{l+1}^{(a)}(\mu)=L_{l}^{(a)} .
$$

Then for $a<n-2$ we have

$$
-P_{l-1}^{(a)}(\nu)+2 P_{l}^{(a)}(\nu)-P_{l+1}^{(a)}(\nu)=L_{l}^{(a)}+m_{l}^{(a-1)}(\nu)-2 m_{l}^{(a)}(\nu)+m_{l}^{(a+1)}(\nu) .
$$

Since $L_{l}^{(a)} \geq 0$, we obtain the assertion. Similarly we can show the assertions for $n-2 \leq a$.

A nice property about these convexity relations is that they do not involve explicitly the information on $L_{l}^{(a)}$ or $\mu$. Indeed, we will not refer to $L_{l}^{(a)}$ or $\mu$ explicitly during the arguments of Sections 5, 6 and 7.

\subsection{Rigged configuration bijection}

Let us define the bijection between the rigged configurations and tensor products of crystals expressed by the KR tableaux which we call paths. For the map from the rigged configuration to paths, the basic operation is called $\delta$

$$
\delta:(\nu, J) \longmapsto\left\{\left(\nu^{\prime}, J^{\prime}\right), k\right\},
$$

where $(\nu, J)$ and $\left(\nu^{\prime}, J^{\prime}\right)$ are rigged configurations and $k \in\{1,2, \ldots, n, \bar{n}, \ldots, \overline{2}, \overline{1}\}$. In the description, we call a string $\left(l, x_{l}\right)$ of $(\nu, J)^{(a)}$ singular if we have $x_{l}=P_{l}^{(a)}(\nu)$, that is, the rigging takes the maximal possible value. To begin with we consider the generic case (non-spin case). Necessary modifications related with the spin cases will be given in Section 4.5.

Definition 3.14. Let us consider a rigged configuration $(\nu, J)$ corresponding to the tensor product of the form $B^{a, l} \otimes B^{\prime}$ (equivalently, $\mu^{(a)}$ has length $l$ row). For $1 \leq a \leq n-2$, the $\operatorname{map} \delta_{l}^{(a)}$

$$
\delta_{l}^{(a)}:(\nu, J) \longmapsto\left\{\left(\nu^{\prime}, J^{\prime}\right), k\right\}
$$

is defined by the following procedure. Set $\ell^{(a-1)}=l$.

(1) For $a \leq i \leq n-2$, assume that $\ell^{(i-1)}$ is already determined. Then we search for the shortest singular string in $(\nu, J)^{(i)}$ that is longer than or equal to $\ell^{(i-1)}$. 
(a) If there exists such a string, set $\ell^{(i)}$ to be the length of the selected string and continue the process recursively. If there is more than one such string, choose any of them.

(b) If there is no such string, set $\ell^{(i)}=\infty, k=i$ and stop.

(2) Suppose that $\ell^{(n-2)}<\infty$. Then we search for the shortest singular string in $(\nu, J)^{(n-1)}$ (resp. $\left.(\nu, J)^{(n)}\right)$ that is longer than or equal to $\ell^{(n-2)}$ and define $\ell^{(n-1)}\left(\right.$ resp. $\left.\ell^{(n)}\right)$ similarly.

(a) If $\ell^{(n-1)}=\infty$ and $\ell^{(n)}=\infty$, set $k=n-1$ and stop.

(b) If $\ell^{(n-1)}<\infty$ and $\ell^{(n)}=\infty$, set $k=n$ and stop.

(c) If $\ell^{(n-1)}=\infty$ and $\ell^{(n)}<\infty$, set $k=\bar{n}$ and stop.

(d) If $\ell^{(n-1)}<\infty$ and $\ell^{(n)}<\infty$, set $\ell_{(n-1)}=\max \left\{\ell^{(n-1)}, \ell^{(n)}\right\}$ and continue.

(3) For $1 \leq i \leq n-2$, assume that $\ell_{(i+1)}$ is already defined. Then we search for the shortest singular string in $(\nu, J)^{(i)}$ that is longer than or equal to $\ell_{(i+1)}$ and has not yet been selected as $\ell^{(i)}$. Define $\ell_{(i)}$ similarly. If $\ell_{(i)}=\infty$, set $k=\overline{i+1}$ and stop. Otherwise continue. If $\ell_{(1)}<\infty$, set $k=\overline{1}$ and stop.

(4) Once the process has stopped, remove the rightmost box of each selected row specified by $\ell^{(i)}$ or $\ell_{(i)}$. The result gives the output $\nu^{\prime}$.

(5) Define the new riggings $J^{\prime}$ as follows. For the rows that are not selected by $\ell^{(i)}$ or $\ell_{(i)}$, take the corresponding riggings from $J$. In order to define the remaining riggings, replace one $B^{a, l}$ in $B$ by $B^{a-1,1} \otimes B^{a, l-1}$ (equivalently, replace one of the length $l$ row of $\mu^{(a)}$ by a length $(l-1)$ row and add a length 1 row to $\left.\mu^{(a-1)}\right)$. Denote the result by $B^{\prime}$. Use $B^{\prime}$ to compute all the vacancy numbers for $\nu^{\prime}$. Then the remaining riggings are defined so that all the corresponding rows become singular with respect to the new vacancy number.

We remark that the resulting rigged configuration $\left(\nu^{\prime}, J^{\prime}\right)$ is associated with the tensor product $B^{\prime}$. We write $\delta_{2} \delta_{1}(\nu, J)$ etc. for repeated applications of $\delta$ on the rigged configurations.

Definition 3.15. For a given rigged configuration $(\nu, J)$ corresponding to the tensor product of the form $B=B^{r_{1}, s_{1}} \otimes B^{r_{2}, s_{2}} \otimes \cdots \otimes B^{r_{L}, s_{L}}$, define the map $\Phi_{B}$ (sometimes also just denoted $\Phi$ )

$\Phi_{B}:(\nu, J) \longmapsto b$

as follows.

(1) Suppose that $\delta_{1}^{(1)} \cdots \delta_{1}^{\left(r_{1}-1\right)} \delta_{s_{1}}^{\left(r_{1}\right)}(\nu, J)=\left(\nu^{\prime}, J^{\prime}\right)$ yields the sequence of letters $k^{\left(r_{1}\right)}, k^{\left(r_{1}-1\right)}$, $\ldots, k^{(1)}\left(k^{(a)}\right.$ corresponds to $\left.\delta^{(a)}\right)$. Put the transpose of the row

\begin{tabular}{|l|l|l|l|}
\hline$k^{(1)}$ & $k^{(2)}$ & $\ldots$ & $k^{\left(r_{1}\right)}$ \\
\hline
\end{tabular}

as the leftmost column of the rectangle $\left(s_{1}^{r_{1}}\right)$.

(2) Continue the previous step for $\delta_{1}^{(1)} \cdots \delta_{1}^{\left(r_{1}-1\right)} \delta_{s_{1}-1}^{\left(r_{1}\right)}\left(\nu^{\prime}, J^{\prime}\right)=\left(\nu^{\prime \prime}, J^{\prime \prime}\right)$ and fill the second column for the rectangle $\left(s_{1}^{r_{1}}\right)$ with the produced letters. Repeat the process until all places of $\left(s_{1}^{r_{1}}\right)$ are filled.

(3) Repeat the previous two steps for the remaining rectangles $\left(s_{2}^{r_{2}}\right),\left(s_{3}^{r_{3}}\right), \ldots,\left(s_{L}^{r_{L}}\right)$.

Remark 3.16. The inverse procedure $\Phi^{-1}$ is obtained by reversing all procedure in $\Phi$ step by step. As an example let us give a sketch of the algorithm for the operation $\left(\delta_{1}^{(1)}\right)^{-1}$. Suppose that we start from an unbarred letter $k$ in a KR tableau. Then we look for the largest singular string of $\nu^{(k)}$. If there is no such string, add a length one row to the bottom of $\nu^{(k)}$. Otherwise add a box to the longest singular row of $\nu^{(k)}$. Suppose that we add the box to the $\ell^{(k)}$ th column 
of $\nu^{(k)}$. Then we look for the singular string of $\nu^{(k-1)}$ which is strictly shorter than $\ell^{(k)}$. We continue this process up to $\nu^{(1)}$. Finally change the riggings for the modified strings according to the new vacancy numbers. Here remind that we have to add a length one row to $\mu^{(1)}$ for the computation of the vacancy numbers.

The following fundamental result is conjectured in [24].

Conjecture 3.17. Consider a tensor product of crystals $B=B^{r_{1}, s_{1}} \otimes B^{r_{2}, s_{2}} \otimes \cdots \otimes B^{r_{L}, s_{L}}$. Then the map $\Phi_{B}$ gives a well-defined bijection between the rigged configuration of type $B$ and the elements of $B$ expressed via the $K R$ tableaux representation.

Currently this conjecture is verified in the following cases:

- $B=B^{r_{1}, s_{1}} \otimes B^{r_{2}, s_{2}} \otimes \cdots \otimes B^{r_{L}, s_{L}}$ type tensor products of $A_{n}^{(1)}[19]$,

- $B=B^{r_{1}, 1} \otimes B^{r_{2}, 1} \otimes \cdots \otimes B^{r_{L}, 1}$ type tensor products of $D_{n}^{(1)}[31]$,

- $B=B^{1, s_{1}} \otimes B^{1, s_{2}} \otimes \cdots \otimes B^{1, s_{L}}$ type tensor products of $D_{n}^{(1)}[34]$,

- $B=B^{r, s}(r<n-1)$ case of $D_{n}^{(1)}[24]$.

A proof for the general case $B=B^{r_{1}, s_{1}} \otimes B^{r_{2}, s_{2}} \otimes \cdots \otimes B^{r_{L}, s_{L}}$ of type $D_{n}^{(1)}$ is the subject of [25]. However, since the paper [25] is yet not available for public, we will not assume this result in this paper.

If there is no afraid of confusion, we use the abbreviation $\Phi$ instead of $\Phi_{B}$.

Example 3.18. Let us consider the following rigged configuration corresponding to the tensor product $B^{2,2} \otimes B^{3,2}$ of type $D_{5}^{(1)}$ :
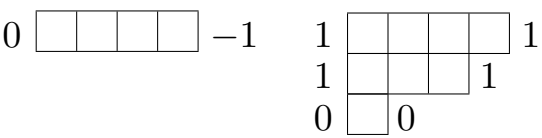

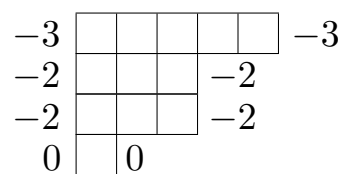

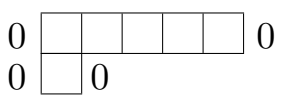

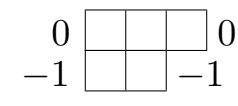

We start by removing $B^{2,2}$ part. Then the first operation is $\delta_{2}^{(2)}$ which start by searching the shortest singular string of $\nu^{(2)}=(4,3,1)$ whose length is equal to or larger than 2 . We work out all the procedure corresponding to $B^{2,2}$ in the following sequence of diagrams
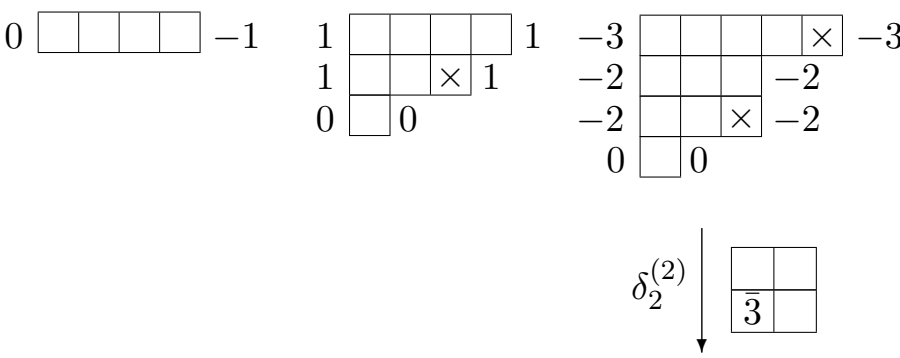

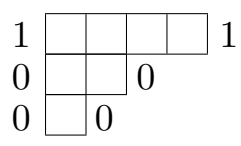

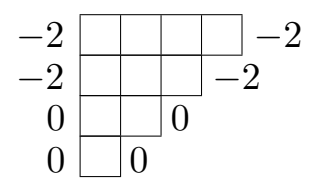

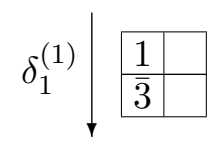

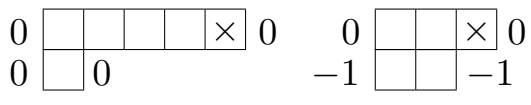

0

0
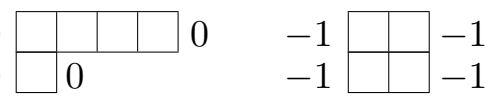


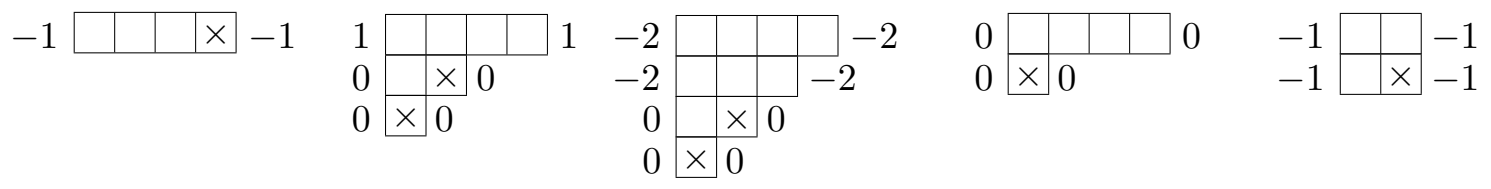

$$
\begin{aligned}
& \delta_{1}^{(2)} \downarrow \begin{array}{|l|l|}
\hline \frac{1}{3} & \\
\hline 3 & \overline{1} \\
\hline
\end{array}
\end{aligned}
$$

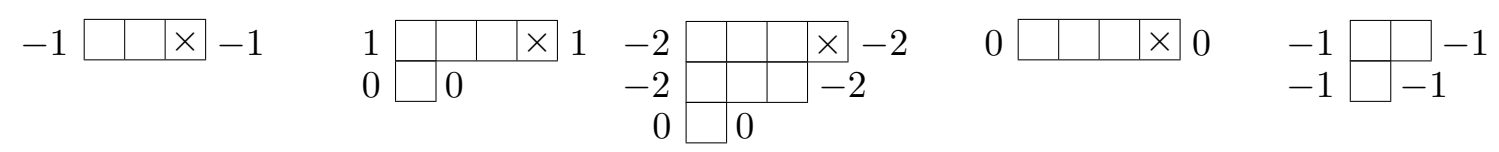

$$
\begin{aligned}
& \delta_{1}^{(1)} \downarrow \begin{array}{|l|l|}
\hline 1 & 5 \\
\hline 3 & \overline{1} \\
\hline
\end{array}
\end{aligned}
$$

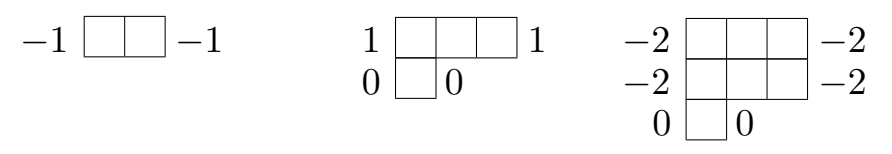
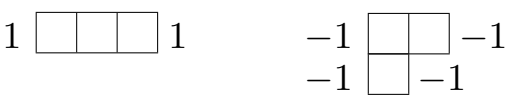

Here the boxes to be removed by $\delta_{l}^{(a)}$ indicated below the corresponding rigged configurations are marked by " $\times$ ". The output of each $\delta_{l}^{(a)}$ is given on the right of the corresponding arrows. Note that after $\delta_{2}^{(2)}$ we recalculate all the vacancy numbers assuming that the resulting rigged configuration corresponds to the tensor product of type $B^{1,1} \otimes B^{2,1} \otimes B^{3,2}$. We remark that the first $\delta_{1}^{(1)}$ cannot remove a box from $\nu^{(1)}$ since there is no singular string and, $\delta_{1}^{(2)}$ starts by removing from $\nu^{(2)}$ and ends at $\nu^{(1)}$. The final output for the given rigged configuration $(\nu, J)$ is as follows:

$$
\Phi_{B^{2,2} \otimes B^{3,2}}(\nu, J)=\begin{array}{|l|l|l|l|}
\hline 1 & 5 \\
\hline \overline{3} & \overline{1} \\
\hline 4 & \overline{5} \\
\hline 4 & \overline{3} \\
\hline 5 & \overline{1} \\
\hline
\end{array}
$$

Remark 3.19. In the above example, we can reverse the order of the tensor product. In this case, we obtain

$$
\Phi_{B^{3,2} \otimes B^{2,2}}(\nu, J)=\begin{array}{|l|l|}
\hline 1 & 4 \\
\hline 5 & \overline{2} \\
\hline \overline{3} & \overline{1} \\
\hline
\end{array} \quad \otimes \begin{array}{|l|l|}
\hline 2 & \overline{5} \\
\hline 5 & \overline{3} \\
\hline
\end{array}
$$

Then the two tensor products in (3.4) and (3.5) are isomorphic under the combinatorial $R$-matrix

$$
R: \begin{array}{|l|l|l|l|}
\hline 1 & 5 \\
\hline \overline{3} & \overline{1} \\
\hline 1 & \overline{5} \\
\hline 4 & \overline{3} \\
\hline 5 & \overline{1} \\
\hline
\end{array} \longmapsto \begin{array}{|l|l|}
\hline 1 & 4 \\
\hline 5 & \overline{2} \\
\hline \overline{3} & \overline{1} \\
\hline
\end{array} \otimes \begin{array}{|l|l|}
\hline 2 & \overline{5} \\
\hline 5 & \overline{3} \\
\hline
\end{array}
$$

Therefore in this case we have $R=\Phi_{B^{3,2} \otimes B^{2,2}} \circ \Phi_{B^{2,2} \otimes B^{3,2}}^{-1}$. This relation is valid not only for two times tensor product but also for multiple applications of pairwise combinatorial $R$-matrices for arbitrary tensor products. 


\section{Rigged configurations and the Kashiwara operators}

\subsection{Statement of the main result}

Let us state the main result of the present paper.

Theorem 4.1. Suppose that the rigged configuration bijection $\Phi$ is well-defined. Then the bijection $\Phi$ and the Kashiwara operators $\widetilde{e}_{i}$ and $\widetilde{f}_{i}\left(i \in I_{0}\right)$ of types $A_{n}^{(1)}$ and $D_{n}^{(1)}$ satisfy the following commutative diagrams:

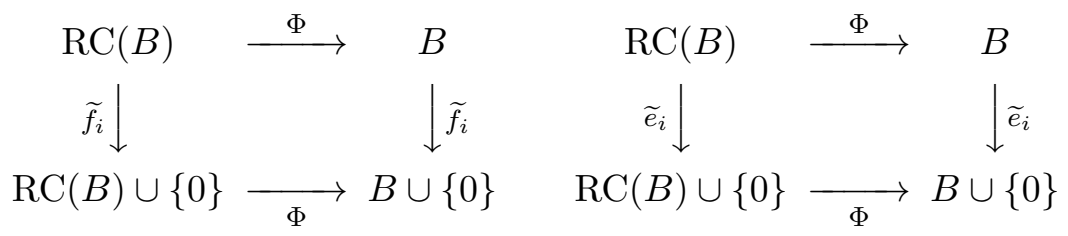

Here for the tensor product of crystals $B=B^{r_{L}, s_{L}} \otimes B^{r_{L-1}, s_{L-1}} \otimes \cdots \otimes B^{r_{1}, s_{1}}$, we denote by $\mathrm{RC}(B)$ the rigged configurations which are mapped to elements of $B$ under $\Phi_{B}$, that is, $\Phi_{B}: \mathrm{RC}(B) \rightarrow B$.

Remark 4.2. Note that $\mathrm{RC}(B)$ does not fully depend on $B$ but depends only on the shape of $B$ via the quantities $L_{l}^{(a)}$ or $\mu$. Thus it is possible to denote the set of rigged configurations as $\operatorname{RC}(L(B))$.

Therefore we have the compatibility of the rigged configuration bijection and the classical Kashiwara operators. We give a few words on the compatibility for the affine Kashiwara operators $\widetilde{e}_{0}$ and $\widetilde{f}_{0}$ of type $D_{n}^{(1)}$. Recall that in [33] the operators $\widetilde{e}_{0}$ and $\widetilde{f}_{0}$ are realized via the bijection between the set of $J$-highest element of $B^{r, s}$ and the combinatorial objects called the plus-minus diagrams. Here $J=\{2,3, \ldots, n\}$. In [24, Section 4], we introduce an analogue of the plus-minus diagrams on the set of the rigged configurations and show that this indeed yields the affine isomorphism with the $\mathrm{KR}$ crystal $B^{r, s}$.

The main points of the arguments in [24] are as follows. In [24, Theorem 5.9] we prove the combinatorial bijection $\Phi$ for the $I_{0}$-highest element of $B^{r, s}$ directly. Then in [24, Proposition 4.3] we show that the same sequence of the classical Kashiwara operators $\tilde{f}_{i}$ gives the plus-minus diagram and the corresponding element in the set of rigged configuration. Therefore Theorem 4.1 asserts that the rigged configuration analogue of the plus-minus diagrams introduced in $[24$, Section 4] are indeed the image of the plus-diagrams under the rigged configuration bijection $\Phi$. Hence, in view of the construction of [33], we have the compatibility of the affine Kashiwara operators $\widetilde{e}_{0}, \widetilde{f}_{0}$ and the rigged configuration bijection for the case $B^{r, s}$. However, the compatibility of the affine Kashiwara operators and the combinatorial bijection $\Phi$ for more than two tensor factors is still an open problem.

The rest of the paper is devoted to the proof of Theorem 4.1.

\subsection{Preliminary steps}

Our main strategy of the proof is to reduce the essential combinatorial aspects of Theorem 4.1 to the fundamental operation $\delta=\delta_{1}^{(1)}$. For this purpose we decompose the original bijection $\Phi$ into several steps according to the operations $\mathrm{lh}, \mathrm{lb}$ and $\mathrm{ls}$ defined on the KR tableau representation of the crystals. In the following, we define the corresponding operations (summarized in the following table) on the set of the rigged configurations.

\begin{tabular}{|c|c|}
\hline$B$ & $\mathrm{RC}(B)$ \\
\hline \hline $\mathrm{ls}$ & $\gamma$ \\
$\mathrm{lb}$ & $\beta$ \\
$\mathrm{lh}$ & $\delta$ \\
\hline
\end{tabular}


First, let us remind that the following symbols bear specific meanings throughout the paper.

- $\nu$ : shapes of the configurations,

- $J$ : riggings,

- $\mu$ : shape of the tensor product.

Definition 4.3. Let $B=B^{r_{L}, s_{L}} \otimes B^{r_{L-1}, s_{L-1}} \otimes \cdots \otimes B^{r_{1}, s_{1}}$.

(1) Corresponding to $B^{r_{L}, s_{L}}=B^{a, l}, \gamma$ replaces a length $l$ row of $\mu^{(a)}$ by rows of lengths $l-1$ and 1.

(2) Suppose that $B^{r_{L}, s_{L}}=B^{r, 1}$ where $r>1$. If $r<n-1$, then $\beta$ removes a length one row from $\mu^{(r)}$, adds a length one row to each of $\mu^{(1)}$ and $\mu^{(r-1)}$ and adds a length one singular string to each of $(\nu, J)^{(a)}$ for $a<r$.

(3) Suppose that $B^{r_{L}, s_{L}}=B^{1,1}$. Then define $\delta=\delta_{1}^{(1)}$.

Proposition 4.4. We can decompose the map $\Phi$ into the following three steps.

(1) Let $B=B^{r, s} \otimes \bar{B}$ with $s>1$. Then we reduce the problem to the case of $B^{r, 1}$ by

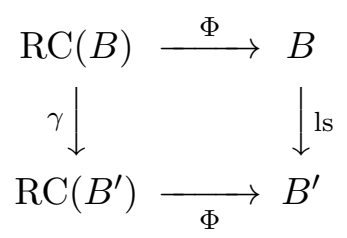

where $B^{\prime}=B^{r, 1} \otimes B^{r, s-1} \otimes \bar{B}$.

(2) Let $B=B^{r, 1} \otimes \bar{B}$ with $r>1$. Then we reduce the problem to the case of $B^{1,1}$ by

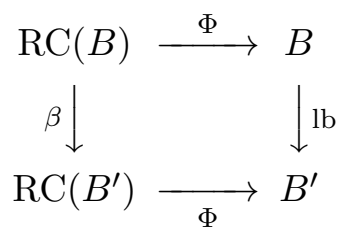

where $B^{\prime}=B^{1,1} \otimes B^{r-1,1} \otimes \bar{B}$.

(3) Let $B=B^{1,1} \otimes \bar{B}$. Then we have to deal with the fundamental operation $\delta$ as

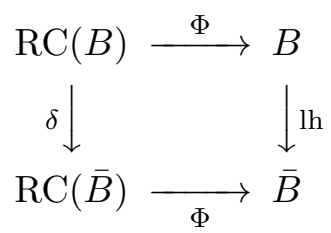

Proof. (1) Suppose that $\Phi$ maps $(\nu, J) \in \mathrm{RC}(B)$ to $b \otimes \bar{b} \in B$. Recall that $\gamma$ replaces a length $s$ row of $\mu^{(r)}$ by rows of lengths $s-1$ and 1 . Thus after application of $\gamma$ there is no singular string of $\nu^{(r)}$ which are strictly shorter than $s$. Then the length 1 row of $\mu^{(r)}$ corresponds to the same column which appear as the leftmost column of the tableau $b$.

(2) If we apply $\delta_{1}^{(1)}$ for $\mathrm{RC}\left(B^{\prime}\right)$, it automatically selects the length 1 singular strings of $\nu^{(a)}$ $(a<r)$ created by $\beta$. As for $\nu^{(r)}$, since $\beta$ removes length 1 row from $\mu^{(r)}$ and adds a length 1 row to $\nu^{(r-1)}$, all the coriggings for $\nu^{(r)}$ do not change after $\beta$. Thus $\delta_{1}^{(1)}$ selects the same string which is selected by $\delta_{1}^{(r)}$ before $\beta$.

(3) This follows from the definition. 


\subsection{Reduction steps}

We show that the operations $\gamma$ and $\beta$ commutes with the Kashiwara operators.

Proposition 4.5. Let $B=B^{r, s} \otimes \bar{B}$ where $s>1$. Then we have the following commutative diagrams

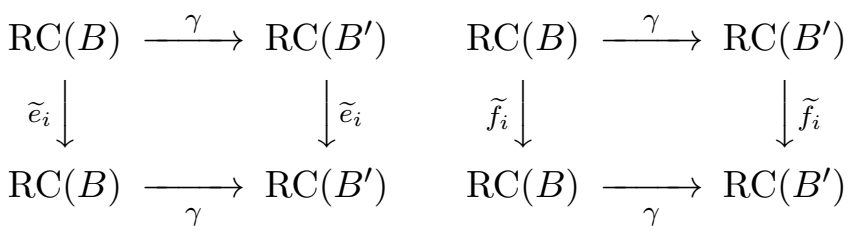

where $B^{\prime}=B^{r, 1} \otimes B^{r, s-1} \otimes \bar{B}$ and $i \in I_{0}$.

Proof. For the rigged configuration $\{\mu,(\nu, J)\}, \widetilde{e}_{i}$ and $\widetilde{f}_{i}$ change $(\nu, J)$ part only and $\gamma$ changes $\mu$ part only. Thus they commute.

We remark that if we have $\widetilde{e}_{i}(\nu, J)=0$ or $\widetilde{f}_{i}(\nu, J)=0$, we have $\widetilde{e}_{i} \circ \gamma(\nu, J)=0$ or $\tilde{f}_{i} \circ \gamma(\nu, J)=0$ by Theorem 3.8 since $\gamma$ does not alter the vacancy numbers nor riggings.

Proposition 4.6. Let $B=B^{r, 1} \otimes \bar{B}$ where $r>1$. Then we have the following commutative diagrams

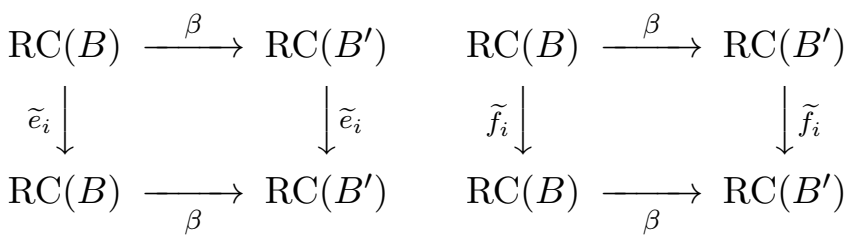

where $B^{\prime}=B^{1,1} \otimes B^{r-1,1} \otimes \bar{B}$ and $i \in I_{0}$.

Proof. Let us prove the $\tilde{f}_{i}$ part first. During the proof we denote that $\widetilde{f}_{i}(\nu, J)=(\widetilde{\nu}, \widetilde{J})$ and $\beta(\nu, J)=(\dot{\nu}, \dot{J})$.

Suppose that $i>r$. Recall that the operation $\beta$ does nothing on $(\nu, J)^{(a)}$ for $a \geq r$. Thus $\beta$ and $\widetilde{f}_{i}$ commute since they do not have interaction.

Suppose that $i=r$. Since the operation $\beta$ adds a length one string to $(\nu, J)^{(r-1)}$, the interaction between $\beta$ and $\widetilde{f}_{r}$ can occur if $\widetilde{f}_{r}$ adds the string $(1,-1)$ to $(\nu, J)^{(r)}$. In this case, since $\beta$ does nothing on $(\nu, J)^{(r)}, \widetilde{f}_{r}$ adds the same string $(1,-1)$ after $\beta$. Let us consider $(\nu, J)^{(r-1)}$. Then we have to check the coincidence of the string added by $\beta$ :

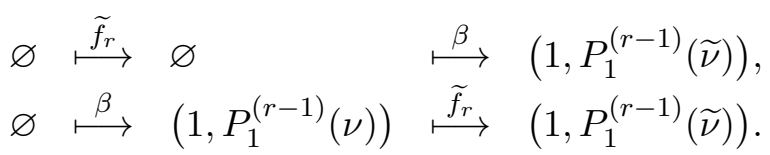

Here we use the fact that $\widetilde{f}_{r}$ changes the riggings so as to keep the coriggings.

Finally, suppose that $i<r$. Let $x_{k}$ be the smallest rigging of length $k$ strings of $(\nu, J)^{(i)}$. Let $x_{\ell}$ be the smallest rigging of $(\nu, J)^{(i)}$ where $\ell$ is the length of the largest string with rigging $x_{\ell}$.

Suppose that $\nu^{(i)} \neq \varnothing$ and $x_{\ell} \leq 0$. Recall that $\beta$ adds the singular string $\left(1, P_{1}^{(i)}(\dot{\nu})\right)$ to $(\nu, J)^{(i)}$. If we show

$$
P_{1}^{(i)}(\dot{\nu}) \geq x_{\ell}
$$

then we see that $\tilde{f}_{i}$ chooses the same string $\left(\ell, x_{\ell}\right)$ even after $\beta$. Let $j$ be the length of the shortest string of $(\nu, J)^{(i)}$. Suppose that $j=1$. Then we have $P_{1}^{(i)}(\dot{\nu})=P_{1}^{(i)}(\nu)$ by definition 
of $\beta, P_{1}^{(i)}(\nu) \geq x_{1}$ by the definition of the rigged configurations and $x_{1} \geq x_{\ell}$ by the minimality of $x_{\ell}$. Thus we have $P_{1}^{(i)}(\dot{\nu}) \geq x_{\ell}$ in this case. Next, suppose that $j>1$. By the convexity relation of $P_{k}^{(i)}(\nu)$ between $0 \leq k \leq j$ we have

$$
P_{1}^{(i)}(\dot{\nu})=P_{1}^{(i)}(\nu) \geq \min \left\{P_{0}^{(i)}(\nu), P_{j}^{(i)}(\nu)\right\}=\min \left\{0, P_{j}^{(i)}(\nu)\right\} .
$$

If we have $P_{j}^{(i)}(\nu) \geq 0$, we have $P_{1}^{(i)}(\dot{\nu}) \geq 0 \geq x_{\ell}$. On the other hand, suppose that $0>P_{j}^{(i)}(\nu)$. Then we have $P_{1}^{(i)}(\dot{\nu}) \geq P_{j}^{(i)}(\nu)$ by the above inequality, $P_{j}^{(i)}(\nu) \geq x_{j}$ by the definition of the rigged configurations and $x_{j} \geq x_{\ell}$ by the minimality of $x_{\ell}$. Therefore we have $P_{1}^{(i)}(\dot{\nu}) \geq x_{\ell}$.

Let us consider the case $\nu^{(i)}=\varnothing$ or $x_{\ell}>0$. In both cases, if we have

$$
P_{1}^{(i)}(\dot{\nu})>0
$$

then we have the following behaviors of the two strings of $(\nu, J)^{(i)}$ :

$$
\begin{array}{lllll}
\varnothing & \beta & \left(1, P_{1}^{(i)}(\dot{\nu})\right) \quad \stackrel{\widetilde{f}_{i}}{\longmapsto} & \begin{array}{l}
\left(1, P_{1}^{(i)}(\widetilde{\dot{\nu}})\right) \\
\varnothing
\end{array} \quad & \varnothing
\end{array}
$$

and, on the other hand,

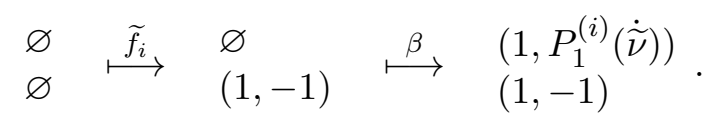

Here we denote by $\widetilde{f}_{i} \circ \beta(\nu, J)=(\widetilde{\bar{\nu}}, \tilde{\dot{J}})$ and $\beta \circ \widetilde{f}_{i}(\nu, J)=(\dot{\tilde{\nu}}, \dot{\widetilde{J}})$. Since $\beta$ does not change vacancy numbers, we have $P_{1}^{(i)}(\widetilde{\dot{\nu}})=P_{1}^{(i)}(\dot{\widetilde{\nu}})$. Thus $\widetilde{f}_{i}$ and $\beta$ commute in this case.

Now let us consider the case $P_{1}^{(i)}(\nu)=P_{1}^{(i)}(\dot{\nu}) \leq 0$.

- Suppose that we have $\nu^{(i)}=\varnothing$. Then we have $P_{\infty}^{(i)}(\nu) \geq 0$ since we have $Q_{\infty}^{(i)}(\nu)=0$. Now we invoke the convexity relation of $P_{k}^{(i)}(\nu)$ between $0 \leq k \leq \infty$ with $0=P_{0}^{(i)}(\nu) \geq$ $P_{1}^{(i)}(\nu) \leq P_{\infty}^{(i)}(\nu)$ to deduce that $P_{0}^{(i)}(\nu)=P_{1}^{(i)}(\nu)=\cdots=P_{\infty}^{(i)}(\nu)=0$. Since $\nu^{(i)}=\varnothing$, this relation also implies that $\nu^{(i-1)}=\nu^{(i+1)}=\mu^{(i)}=\varnothing$.

Consider $\widetilde{f}_{i}$ acting on $(\nu, J)$. Suppose that $i<r-1$. Then it adds the string $(1,-1)$ to $(\nu, J)^{(i)}$ whereas the corresponding vacancy number is $P_{1}^{(i)}(\widetilde{\nu})=P_{1}^{(i)}(\nu)-2=-2$. Thus we have $\tilde{f}_{i}(\nu, J)=0$. On the other hand, $\beta$ acting on $(\nu, J)$ adds the length 1 singular string and gives $(\dot{\nu}, \dot{J})^{(i)}=\left\{\left(1, P_{1}^{(i)}(\dot{\nu})\right)\right\}$. The next $\tilde{f}_{i}$ selects this string since we have $P_{1}^{(i)}(\dot{\nu})=P_{1}^{(i)}(\nu)=0$. Then we have $(\widetilde{\dot{\nu}}, \widetilde{\dot{J}})^{(i)}=\{(2,-1)\}$. In particular we have $Q_{2}^{(i)}(\widetilde{\dot{\nu}})=2$. Since $\nu^{(i-1)}=\nu^{(i+1)}=\varnothing$, both $\widetilde{\dot{\nu}}^{(i-1)}$ and $\widetilde{\dot{\nu}}^{(i+1)}$ consist of length 1 string. Thus $Q_{2}^{(i-1)}(\widetilde{\dot{\nu}})=Q_{2}^{(i+1)}(\widetilde{\dot{\nu}})=1$. Since we also know $\mu^{(i)}=\varnothing$, we have $Q_{2}^{(i)}(\widetilde{\dot{\mu}})=0$. Hence $P_{2}^{(i)}(\widetilde{\dot{\nu}})=Q_{2}^{(i)}(\widetilde{\tilde{\mu}})-2 Q_{2}^{(i)}(\widetilde{\dot{\nu}})+Q_{2}^{(i-1)}(\widetilde{\dot{\nu}})+Q_{2}^{(i+1)}(\widetilde{\dot{\nu}})=-2$. Therefore we have $\tilde{f}_{i} \circ \beta(\nu, J)=0$. Hence $\widetilde{f}_{i}$ and $\beta$ commute in this case. If $i=r-1$, we can use the parallel argument if we replace $\nu^{(i+1)}$ by $\mu^{(i)}$.

- Since we have completed the proof for the case $\nu^{(i)}=\varnothing$, we can assume that $\nu^{(i)} \neq \varnothing$. Let $j$ be the length of the smallest string of $(\nu, J)^{(i)}$. Suppose that $j=1$. Since we are considering the case $\nu^{(i)}=\varnothing$ or $x_{\ell}>0$, the condition $\nu^{(i)} \neq \varnothing$ implies that $x_{\ell}>0$. On the other hand we have $0 \geq P_{1}^{(i)}(\nu) \geq x_{1}$ by the assumption and the definition of the rigged configurations. This is in contradiction to the relation $x_{1} \geq x_{\ell}$ which follows from the minimality of $x_{\ell}$. Hence this case cannot happen. 
- Suppose that $j>1$. Again we have $x_{\ell}>0$. By the convexity relation of $P_{k}^{(i)}(\nu)$ between $0 \leq k \leq j$ and the definition of the rigged configurations we have $0=P_{0}^{(i)}(\nu) \geq P_{1}^{(i)}(\nu) \geq$ $P_{j}^{(i)}(\nu) \geq x_{j}$. This is in contradiction to the relation $x_{j} \geq x_{\ell}$. Hence this case cannot occur.

Thus we have checked the commutativity of $\tilde{f}_{i}$ and $\beta$ in all possible cases.

Finally let us consider the proof for $\widetilde{e}_{i}$. If $\widetilde{e}_{i}(\nu, J) \neq 0$, then the commutativity of $\widetilde{e}_{i}$ and $\beta$ follows from the result of $\widetilde{f}_{i}$ since $\widetilde{e}_{i}$ and $\widetilde{f}_{i}$ are mutually inverse in this case. Suppose that $\widetilde{e}_{i}(\nu, J)=0$. Then all the riggings of $(\nu, J)^{(i)}$ are non-negative. Recall that $\beta$ acting on $(\nu, J)$ adds the string $\left(1, P_{1}^{(i)}(\dot{\nu})\right)$. Then if we show $P_{1}^{(i)}(\dot{\nu})=P_{1}^{(i)}(\nu) \geq 0$ we have $\widetilde{e}_{i} \circ \beta(\nu, J)=0$. Let $j$ be the length of the shortest string of $(\nu, J)^{(i)}$. If $\nu^{(i)}=\varnothing$, set $j=\infty$. In both cases we have $P_{j}^{(i)}(\nu) \geq 0$ since we have $P_{j}^{(i)}(\nu) \geq x_{j} \geq 0$ if $j<\infty$ and $Q_{j}^{(i)}(\nu)=0$ if $j=\infty$. Now we invoke the convexity relation of $P_{k}^{(i)}(\nu)$ between $0 \leq k \leq j$. Then we have $P_{1}^{(i)}(\nu) \geq$ $\min \left\{P_{0}^{(i)}(\nu), P_{j}^{(i)}(\nu)\right\}=\min \left\{0, P_{j}^{(i)}(\nu)\right\}=0$ as desired.

\subsection{Statements to be proved}

Due to the arguments in the previous section, the essential points of the proof of Theorem 4.1 are reduced to the following two assertions on the case $B=B^{1,1} \otimes \bar{B}$. Up to Section 6 from here we will use the following notation when we consider the case $B=B^{1,1} \otimes \bar{B}$. Let $(\nu, J) \in \operatorname{RC}(B)$. Denote the rigged configurations obtained by the actions of $\widetilde{f}_{i}$ or $\delta$ as in the following diagram:

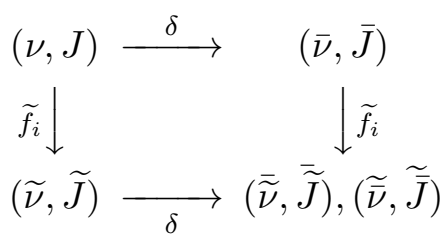

Here we denote as $\delta:(\widetilde{\nu}, \widetilde{J}) \mapsto(\overline{\widetilde{\nu}}, \overline{\widetilde{J}})$ and $\widetilde{f}_{i}:(\bar{\nu}, \bar{J}) \mapsto(\widetilde{\nu}, \widetilde{\bar{J}})$. We denote the smallest rigging associated with length $k$ rows of $\nu^{(i)}$ by $x_{k}$ and express such string as $\left(k, x_{k}\right)$. For later purposes, let us prepare several notation for the specific strings as follows:

- $\left(\ell, x_{\ell}\right)$ is the string of $(\nu, J)$ that is selected by $\tilde{f}_{i}$,

- $\left(\bar{\ell}, x_{\bar{\ell}}\right)$ is the string of $(\bar{\nu}, \bar{J})$ that is selected by $\tilde{f}_{i}$,

- $\ell^{(a)}$ and $\ell_{(a)}$ are the lengths of the singular strings of $(\nu, J)^{(a)}$ which are selected by $\delta$ under the condition $\ell^{(a)} \leq \ell_{(a)}$,

- $\widetilde{\ell}^{(a)}$ and $\widetilde{\ell}_{(a)}$ are the lengths of the singular strings of $(\widetilde{\nu}, \widetilde{J})^{(a)}$ which are selected by $\delta$ under the condition $\widetilde{\ell}^{(a)} \leq \widetilde{\ell}_{(a)}$.

Then our main claims to be proved are the following two assertions which are proved in Sections 5, 6 and 7 .

Proposition 4.7. Let $B=B^{1,1} \otimes \bar{B}$ and $i \in I_{0}$. Then we have the following commutative diagrams

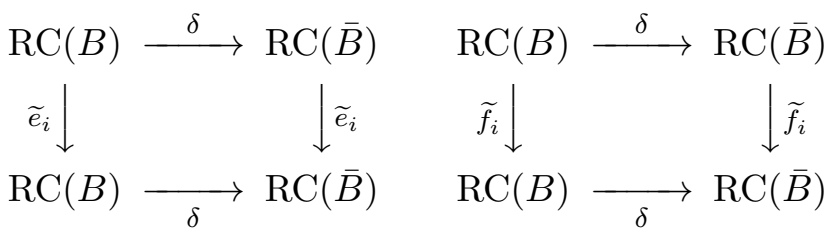

if all $\widetilde{e}_{i}$ and $\widetilde{f}_{i}$ in the above diagrams are defined. 
Proposition 4.8. Let $B=B^{1,1} \otimes \bar{B}, i \in I_{0},(\nu, J) \in \mathrm{RC}(B)$ and $b=b^{\prime} \otimes \bar{b} \in B^{1,1} \otimes \bar{B}$. Suppose that $\Phi:(\nu, J) \longmapsto b$. Then we have the following properties:

(1) $\tilde{f}_{i}(\nu, J) \neq 0$ and $\tilde{f}_{i}(\bar{\nu}, \bar{J})=0 \Longrightarrow \widetilde{f}_{i}(b) \neq 0, \widetilde{f}_{i}(\bar{b})=0$ and $\Phi\left(\widetilde{f}_{i}(\nu, J)\right)=\widetilde{f}_{i}(\Phi(\nu, J))$,

(2) $\tilde{f}_{i}(b) \neq 0$ and $\tilde{f}_{i}(\bar{b})=0 \Longrightarrow \widetilde{f}_{i}(\nu, J) \neq 0, \tilde{f}_{i}(\bar{\nu}, \bar{J})=0$ and $\Phi^{-1}\left(\tilde{f}_{i}(b)\right)=\tilde{f}_{i}\left(\Phi^{-1}(b)\right)$,

(3) $\tilde{f}_{i}(\nu, J)=0$ and $\widetilde{f}_{i}(\bar{\nu}, \bar{J}) \neq 0 \Longrightarrow \widetilde{f}_{i}(b)=0, \widetilde{f}_{i}(\bar{b}) \neq 0$ and $\Phi\left(\widetilde{f}_{i}(\nu, J)\right)=\widetilde{f}_{i}(\Phi(\nu, J))$,

(4) $\tilde{f}_{i}(b)=0$ and $\widetilde{f}_{i}(\bar{b}) \neq 0 \Longrightarrow \widetilde{f}_{i}(\nu, J)=0, \widetilde{f}_{i}(\bar{\nu}, \bar{J}) \neq 0$ and $\Phi^{-1}\left(\widetilde{f}_{i}(b)\right)=\widetilde{f}_{i}\left(\Phi^{-1}(b)\right)$,

(5) $\widetilde{e}_{i}(\nu, J) \neq 0$ and $\widetilde{e}_{i}(\bar{\nu}, \bar{J})=0 \Longrightarrow \widetilde{e}_{i}(b) \neq 0, \widetilde{e}_{i}(\bar{b})=0$ and $\Phi\left(\widetilde{e}_{i}(\nu, J)\right)=\widetilde{e}_{i}(\Phi(\nu, J))$,

(6) $\widetilde{e}_{i}(b) \neq 0$ and $\widetilde{e}_{i}(\bar{b})=0 \Longrightarrow \widetilde{e}_{i}(\nu, J) \neq 0, \widetilde{e}_{i}(\bar{\nu}, \bar{J})=0$ and $\Phi^{-1}\left(\widetilde{e}_{i}(b)\right)=\widetilde{e}_{i}\left(\Phi^{-1}(b)\right)$,

(7) the situation $\widetilde{e}_{i}(\nu, J)=0$ and $\widetilde{e}_{i}(\bar{\nu}, \bar{J}) \neq 0$ cannot happen,

(8) the situation $\widetilde{e}_{i}(b)=0$ and $\widetilde{e}_{i}(\bar{b}) \neq 0$ cannot happen.

Here from (1) to (4) we assume the commutativity of $\widetilde{f}_{i}$ with $\Phi$ for $\bar{B}$ and from (5) to (8) we assume the commutativity of $\widetilde{e}_{i}$ and $\widetilde{f}_{i}$ with $\Phi$ for $\bar{B}$.

Let us show how these properties lead to the proof of Theorem 4.1. In the proof, we will use the diagram of the following kind as in [19]:

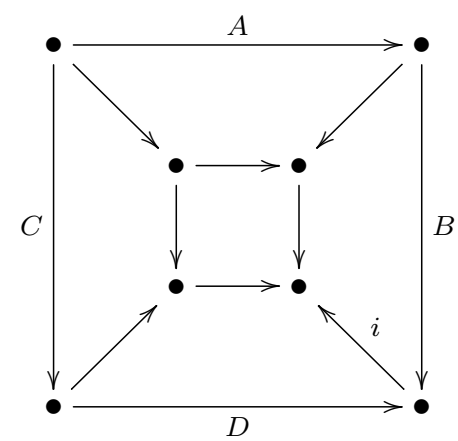

We regard this as a cube with front face given by the large square. Suppose that the square diagrams given by the faces of the cube except for the front face commute and $i$ is the injective map. Then the front face also commutes since we have

$$
i \circ B \circ A=i \circ D \circ C
$$

by a diagram chasing.

Proof of Theorem 4.1. First we prove the $\widetilde{f}_{i}$ case. Then we can prove the $\widetilde{e}_{i}$ case by a parallel argument.

We follow the decomposition of $\Phi$ as described in Proposition 4.4. The simplest case $B^{1,1}$ can be shown directly. We give a list of the explicit rigged configurations in this case:

\begin{tabular}{|l|l|l|}
\hline$b \in B^{1,1}$ & $(\nu, J)^{(a)}$ of $\Phi^{-1}(b)$ & values for $a$ \\
\hline \hline $\bar{i}$ & $\{(1,0)\}$ & $a<i-1$, \\
& $\{(1,-1)\}$ & $a=i-1$ \\
& $\varnothing$ & $i \leq a$. \\
\hline $\bar{n}$ & $\{(1,0)\}$ & $a \leq n-2$, \\
& $\varnothing$ & $a=n-1$ \\
& $\{(1,-1)\}$ & $a=n$ \\
\hline$\overline{\bar{i}}$ & $\{(1,0)\}$ & $a \leq i-2$, \\
$\overline{(i<n)}$ & $\{(1,1)\}$ & $a=i-1$ \\
& $\{(1,-1),(1,-1)\}$ & $a=i$ \\
& $\{(1,0),(1,0)\}$ & $i+1 \leq a \leq n-2$, \\
& $\{(1,0)\}$ & $a=n-1, n$ \\
\hline
\end{tabular}


By using the crystal graph of $B^{1,1}$ presented in Section 2.2 .1 we can verify the commutativity of $\tilde{f}_{i}$ and $\Phi .^{3}$

Let us consider the case $B=B^{1,1} \otimes \bar{B}$. This case corresponds to an addition of a new tensor factor. For this we use the following diagram:

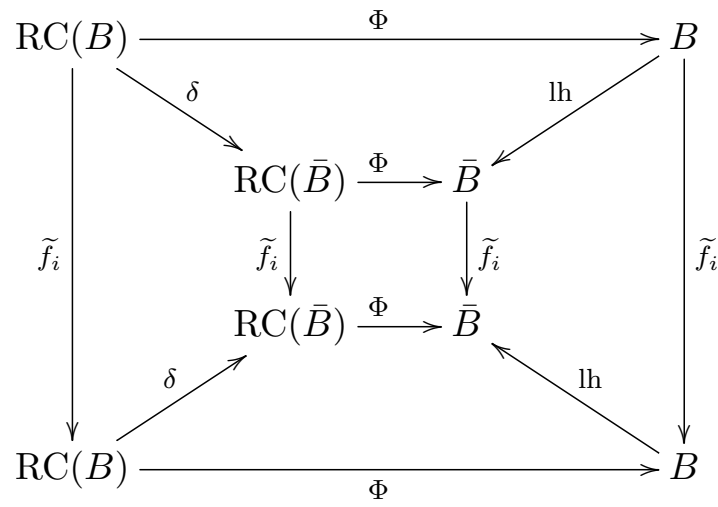

Suppose that all $\tilde{f}_{i}$ which appear in the above diagram are defined. The top face and the bottom face commute by Proposition 4.4. The left face commutes by Proposition 4.7. The right face commutes by definition of $\mathrm{lh}$ on tensor products of crystals. The back face commutes by the assumption. Finally the map $\mathrm{lh}$ is injective if we consider the weight of the element of $B^{1,1}$ subtracted by the operation. Hence the front face commutes. This proves the commutativity of $\Phi$ and $\widetilde{f}_{i}$ when both $\widetilde{f}_{i}$ before or after $\delta$ (or lh) are defined. On the other hand we know from Proposition 4.8 that $\Phi$ and $\widetilde{f}_{i}$ also commute even if one of $\widetilde{f}_{i}$ before or after $\delta$ (or lh) is undefined. By exclusion we have the commutativity for the case when both $\widetilde{f}_{i}$ before and after $\delta$ (or $\mathrm{lh}$ ) are undefined.

Let us consider the the case $B=B^{r, 1} \otimes \bar{B}$ with $r>1$. Let $B^{\prime}=B^{1,1} \otimes B^{r-1,1} \otimes \bar{B}$. Consider the following diagram:

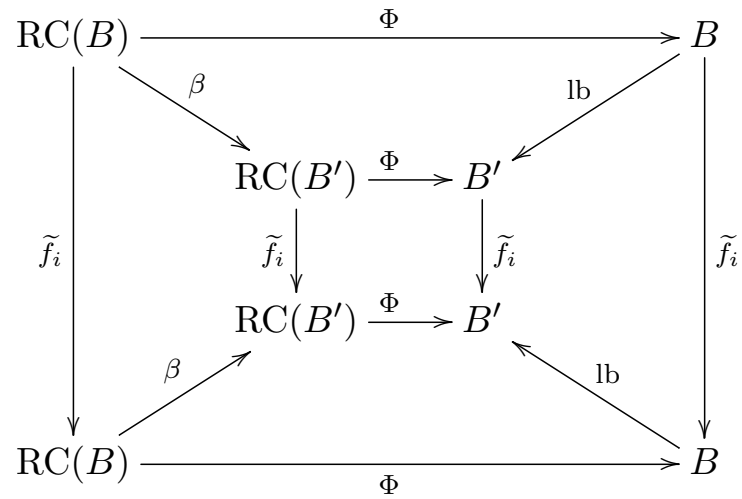

The top face and the bottom face commute by Proposition 4.4. The left face commutes by Proposition 4.6. The right face commutes by definition of $\mathrm{lb}$ on tensor products of crystals. The back face commutes by the assumption. Since lb is injective, the front face commutes.

\footnotetext{
${ }^{3}$ In $B^{1,1}$ case $\Phi$ coincides with $\delta$. In view of the crystal graph of $B^{1,1}$ and the definition of $\widetilde{f}_{i}$ on the rigged configurations we see a connection between the crystal graph and $\delta$ in the case $B^{1,1}$. However this is only the tip of an iceberg. Indeed, in $E_{6}^{(1)}$ case [26], $\delta$ is described by the crystal graph of $B^{1,1}$ as in the present case and the same algorithm can be applied for general tensor products of the form $\left(B^{1,1}\right)^{L}$.
} 
Finally let us consider the the case $B=B^{r, s} \otimes \bar{B}$ with $s>1$. Let $B^{\prime}=B^{r, 1} \otimes B^{r, s-1} \otimes \bar{B}$. Consider the following diagram:

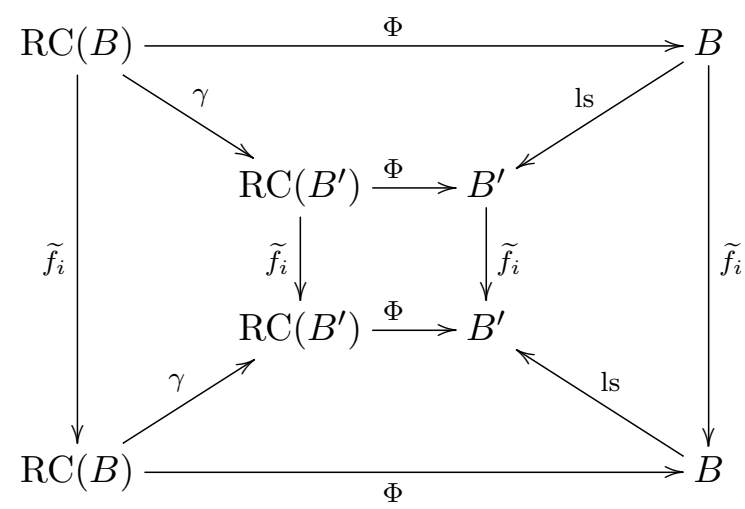

The top face and the bottom face commute by Proposition 4.4. The left face commutes by Proposition 4.5. The right face commutes by the definition of ls on tensor products of crystals. The back face commutes by the assumption. Since ls is injective, the front face commutes. This completes the proof of Theorem 4.1.

\subsection{Spin cases}

Let us consider the arguments which are needed to treat the spin cases. Here we will concentrate on the case $B^{n, s}$. The other case $B^{n-1, s}$ can be obtained by replacing the Dynkin nodes $n$ and $n-1$ in the following description. To begin with, following [31], we introduce the three sets $\hat{B}^{n-1,1}, \hat{B}^{n, 1}$ and $\hat{\bar{B}}^{n, 1}$ which are generated by

$$
\hat{u}_{n-1}=\begin{array}{|c|}
\hline 1 \\
\hline 2 \\
\hline \vdots \\
\hline n-1 \\
\hline
\end{array}
$$
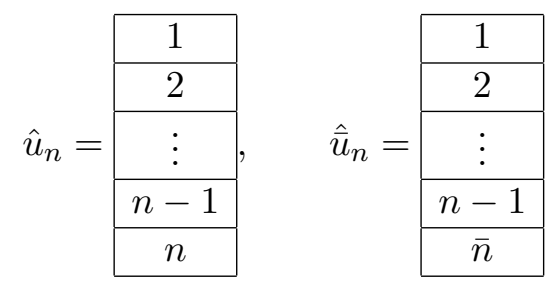

Here we regard them as the usual single columns and apply the Kashiwara operators $\tilde{f}_{i}\left(i \in I_{0}\right)$ as in the case for non-spin KN tableaux to obtain the whole elements of $\hat{B}^{n-1,1}, \hat{B}^{n, 1}$ and $\hat{\bar{B}}^{n, 1}$. On these sets, we define the operation left-box analogously to Definition 2.4. To be more explicit, we have $\mathrm{lb}: \hat{B}^{n, 1} \longrightarrow B^{1,1} \otimes \hat{B}^{n-1,1}$ and $\mathrm{lb}: \hat{B}^{n-1,1} \longrightarrow B^{1,1} \otimes B^{n-2,1}$.

Now we describe the algorithm for the rigged configuration bijection for the spin cases $B^{n-1, l}$ and $B^{n, l}$ following [31]. To begin with, we introduce the embedding of the rigged configurations

$$
\mathrm{emb}:\{\mu,(\nu, J)\} \longmapsto\left\{\mu^{\prime},\left(\nu^{\prime}, J^{\prime}\right)\right\},
$$

where the quantities with primes means that the everything is doubled as $\mu_{i}^{\prime(a)}=2 \mu_{i}^{(a)}, \nu_{i}^{\prime(a)}=$ $2 \nu_{i}^{(a)}$ and $J_{i}^{\prime(a)}=2 J_{i}^{(a)}$. Similarly, if the rigged configuration $\{\mu,(\nu, J)\}$ is composed of even integers, we can define $\mathrm{emb}^{-1}$ by dividing all its components by 2 . Then the operation to obtain the leftmost column of an element of $B^{n, l}$ is

$$
\mathrm{emb}^{-1} \circ \delta_{1}^{(1)} \cdots \delta_{1}^{(n-2)} \hat{\delta}_{1}^{(n-1)} \hat{\delta}_{2}^{(n)} \circ \mathrm{emb} \circ \gamma(\nu, J) .
$$

Here $\gamma$ is the same as Definition 4.3; $\gamma$ replaces one of length $l$ rows of $\mu^{(n)}$ by two rows of length $l-1$ and 1 . The new operations $\hat{\delta}_{2}^{(n)}$ and $\hat{\delta}_{1}^{(n-1)}$ are defined as follows. Let us denote by $\left(\nu^{\prime}, J^{\prime}\right)=\operatorname{emb} \circ \gamma(\nu, J)$. 
- $\hat{\delta}_{2}^{(n)}:\left(\nu^{\prime}, J^{\prime}\right) \longmapsto\left\{\left(\nu^{\prime \prime}, J^{\prime \prime}\right), k\right\}$ is defined as follows. We look for the shortest singular string of $\left(\nu^{\prime}, J^{\prime}\right)^{(n)}$ which is longer than or equal to 2 . If there is no such string, set $\ell^{(n)}=\infty$, $k=n$ and stop. Otherwise we set $\ell_{(n-1)}$ to be the length of the selected string of $\left(\nu^{\prime}, J^{\prime}\right)^{(n)}$ and do the same procedure as in the usual $\delta_{l}^{(a)}$.

The definition of the new rigged configuration $\left(\nu^{\prime \prime}, J^{\prime \prime}\right)$ is the same as in the usual $\delta_{l}^{(a)}$ except for the following point. We replace one of the length 2 rows of $\mu^{\prime(n)}$ by length 1 row and add a length one row to $\mu^{\prime(n-1)}$.

- $\hat{\delta}_{1}^{(n-1)}:\left(\nu^{\prime}, J^{\prime}\right) \longmapsto\left\{\left(\nu^{\prime \prime}, J^{\prime \prime}\right), k\right\}$ is defined as follows. Set $\ell^{(n-2)}=1$. Then, as in the usual $\delta_{l}^{(a)}$ we begin to determine $\ell^{(n-1)}$ and $\ell^{(n)}$ and proceed similarly to the usual case. The new rigged configuration $\left(\nu^{\prime \prime}, J^{\prime \prime}\right)$ is defined similarly as in the usual case except for the following point. We remove a length one row from each of $\mu^{\prime(n-1)}$ and $\mu^{\prime(n)}$ and add a length one row to $\mu^{\prime(n-2)}$.

We regard the output of the sequence of $\delta$ 's as the element of $\hat{B}^{n, 1}$. Corresponding to the final $\mathrm{emb}^{-1}$, we halve the width of the column with keeping all its contents. We regard the final output as the element of $B^{n, 1}$.

Remark 4.9. In the above algorithm, some readers might feel that the change in $\mu$ is peculiar. To get a better understanding of this point, let us take a look at the following computations

$$
\begin{aligned}
& 2 \bar{\Lambda}_{n}=\epsilon_{1}+\cdots+\epsilon_{n-2}+\epsilon_{n-1}+\epsilon_{n}, \\
& \bar{\Lambda}_{n}+\bar{\Lambda}_{n-1}=\epsilon_{1}+\cdots+\epsilon_{n-2}+\epsilon_{n-1}, \\
& \bar{\Lambda}_{n-2}=\epsilon_{1}+\cdots+\epsilon_{n-2} .
\end{aligned}
$$

Let us compare with the special case $\nu^{(a)}=\varnothing$ for all $a \in I_{0}$ with weight $2 \bar{\Lambda}_{n}$ (see (3.2)). In this case we should obtain a column filled by $1,2, \ldots, n$ from top to bottom. Note that each letter $i$ in this column corresponds to $\epsilon_{i}$.

For the proof of Theorem 4.1, we have to modify $\beta$ in Definition 4.3 as follows. $\hat{\beta}^{(n)}$ replaces a length two row of $\mu^{(n)}$ by a length one row, adds a length one row to each of $\mu^{(1)}$ and $\mu^{(n-1)}$ and adds a length one singular string to each of $(\nu, J)^{(a)}$ for $a \leq n-1$. $\hat{\beta}^{(n-1)}$ removes a length one row from each of $\mu^{(n-1)}$ and $\mu^{(n)}$, adds a length one row to each of $\mu^{(1)}$ and $\mu^{(n-2)}$ and adds a length one singular string to each of $(\nu, J)^{(a)}$ for $a \leq n-2$. Note that we have the following property.

Proposition 4.10. On the rigged configurations, we have

$$
\mathrm{emb} \circ \widetilde{e}_{i}=\widetilde{e}_{i}^{2} \circ \mathrm{emb}, \quad \mathrm{emb} \circ \widetilde{f}_{i}=\widetilde{f}_{i}^{2} \circ \mathrm{emb} .
$$

Proof. We prove the $\widetilde{e}_{i}$ part. Let us consider the case $\widetilde{e}_{i}(\nu, J) \neq 0$. Suppose that $\widetilde{e}_{i}$ acts on the string $\left(\ell, x_{\ell}\right)$ of $(\nu, J)^{(i)}$. Then $\widetilde{e}_{i}$ makes the string $\left(2 \ell, 2 x_{\ell}\right)$ of $\operatorname{emb}(\nu, J)^{(i)}$ into $\left(2 \ell-1,2 x_{\ell}+1\right)$. Since $x_{\ell}<0$, we have $2 x_{\ell}+1<0$ and thus $\widetilde{e}_{i}^{2} \circ \operatorname{emb}(\nu, J) \neq 0$. We show that the second $\widetilde{e}_{i}$ acts on the string $\left(2 \ell-1,2 x_{\ell}+1\right)$.

(a) Suppose that there is a string $\left(k, x_{k}\right)$ with $k<\ell$ in $(\nu, J)^{(i)}$. By the minimality of $\ell$ we have $x_{k}>x_{\ell}$. Therefore we have $2 x_{k}>2 x_{\ell}+1$.

(b) Suppose that there is a string $\left(k, x_{k}\right)$ with $\ell \leq k$ in $(\nu, J)^{(i)}$. Then in the $\widetilde{e}_{i} \circ \operatorname{emb}(\nu, J)^{(i)}$, the string becomes $\left(2 k, x_{k}+2\right)$. By the minimality of $x_{\ell}$, we have $x_{k}+2>x_{\ell}+1$.

Therefore the second $\widetilde{e}_{i}$ acts on the string $\left(2 \ell-1,2 x_{\ell}+1\right)$ to make $\left(2 \ell-2,2 x_{\ell}+2\right)$. Thus emb $\circ \widetilde{e}_{i}=\widetilde{e}_{i}^{2} \circ \mathrm{emb}$ in this case. On the other hand, if $\widetilde{e}_{i}(\nu, J)=0$, we have $\widetilde{e}_{i} \circ \operatorname{emb}(\nu, J)=0$ by Theorem 3.8.

Similarly we can show emb $\circ \tilde{f}_{i}=\widetilde{f}_{i}^{2} \circ \mathrm{emb}$. 
Then we can use the same arguments of the proof of Proposition 4.6 to show that $\hat{\beta}^{(n-1)}$ and $\hat{\beta}^{(n)}$ commute with $\widetilde{e}_{i}$ and $\widetilde{f}_{i}$. Thus by using the same arguments we can show Theorem 4.1 in this case.

\section{Proof of Proposition 4.7}

\subsection{Outline}

We concentrate on the proof for $\widetilde{f}_{i}$ part since the other part $\widetilde{e}_{i}$ follows from the former case. We divide the proof into the following five cases which exhaust all the possibilities:

Case A: $\ell+1<\ell^{(i-1)}$,

Case B: $\ell^{(i-1)} \leq \ell+1<\ell^{(i)}=\infty$,

Case $\mathrm{C}: \quad \ell^{(i-1)} \leq \ell+1 \leq \ell^{(i)}<\infty$,

Case D: $\ell^{(i)}=\ell$,

Case E: $\quad \ell^{(i)}<\ell$.

The actual proof for each case is based on the following behaviors of the vacancy numbers induced by $\delta$.

Lemma 5.1. For $1 \leq i \leq n$ we have

$$
P_{\ell^{(i)}-1}^{(i)}(\bar{\nu})= \begin{cases}P_{\ell^{(i)}-1}^{(i)}(\nu) & \left(\text { if } \ell^{(i-1)}=\ell^{(i)}\right), \\ P_{\ell^{(i)}-1}^{(i)}(\nu)-1 & \left(\text { if } \ell^{(i-1)}<\ell^{(i)}\right) .\end{cases}
$$

Here if $i=n$ we understand $\ell^{(i-1)}$ as $\ell^{(n-2)}$. For $1 \leq i \leq n-3$ we have

$$
P_{\ell_{(i)}-1}^{(i)}(\bar{\nu})= \begin{cases}P_{\ell_{(i)}-1}^{(i)}(\nu) & \left(\text { if } \ell^{(i-1)}=\ell^{(i)}=\cdots=\ell_{(i)}\right), \\ P_{\ell_{(i)}-1}^{(i)}(\nu)-1 & \left(\text { if } \ell^{(i-1)}<\ell^{(i)}=\cdots=\ell_{(i)}\right), \\ P_{\ell_{(i)}-1}^{(i)}(\nu)+1 & \left(\text { if } \ell^{(i)}<\ell^{(i+1)}=\cdots=\ell_{(i)}\right) \\ P_{\ell_{(i)}-1}^{(i)}(\nu) & \left(\text { if } \ell^{(i+1)}<\ell_{(i+1)}=\ell_{(i)}\right) \\ P_{\ell_{(i)}-1}^{(i)}(\nu)-1 & \left(\text { if } \ell_{(i+1)}<\ell_{(i)}\right)\end{cases}
$$

and for $i=n-2$ we have

$$
P_{\ell_{(n-2)}^{(n-2)}}^{(\bar{\nu})}=\left\{\begin{array}{lll}
P_{\ell_{(n-2)}^{(n-2)}}^{(n)} & \left(\text { if } \ell^{(n-3)}=\ell^{(n-2)}=\ell^{(n-1)}=\ell^{(n)}=\ell_{(n-2)}\right), & \text { (VIII) } \\
P_{\ell_{(n-2)}-1}^{(n-2)}(\nu)-1 & \left(\text { if } \ell^{(n-3)}<\ell^{(n-2)}=\ell^{(n-1)}=\ell^{(n)}=\ell_{(n-2)}\right), & \text { (IX) } \\
P_{\ell_{(n-2)}(n-1)}^{(n)}(\nu) & \left(\text { if } \ell^{(n-2)}<\ell^{(n-1)}=\ell^{(n)}=\ell_{(n-2)}\right), & \text { (X) } \\
P_{\ell_{(n-2)}^{(n-2)}-1}^{(\nu)} & \left(\text { if } \min \left\{\ell^{(n-1)}, \ell^{(n)}\right\}<\ell_{(n-1)}=\ell_{(n-2)}\right), & \text { (XI) } \\
P_{\ell_{(n-2)}^{(n-2)}-1}(\nu)-1 & \left(\text { if } \ell_{(n-1)}<\ell_{(n-2)}\right) . & \text { (XII) }
\end{array}\right.
$$

During the proof, the cases (VIII) to (XII) can be treated as the special case of the cases (III) to (VII). Indeed, we can assume that $\ell^{(n-1)} \leq \ell^{(n)}$ without loss of the generality. Then we have $\ell_{(n-1)}=\ell^{(n)}$ and regard $\ell^{(n-1)}$ and $\ell_{(n-1)}$ as lengths of strings of the concatenation $\left(\left(\nu^{(n-1)}, \nu^{(n)}\right),\left(J^{(n-1)}, J^{(n)}\right)\right)$ of $(\nu, J)^{(n-1)}$ and $(\nu, J)^{(n)}$.

For the reader's convenience, we give a summary of all major subcases here. 
$\mathrm{A} \ell+1<\ell^{(i-1)}$.

$\mathrm{B} \ell^{(i-1)} \leq \ell+1<\ell^{(i)}=\infty$,

(1) $\tilde{f}_{i}$ creates a non-singular string,

(2) $\tilde{f}_{i}$ creates a singular string.

$\mathrm{C} \ell^{(i-1)} \leq \ell+1 \leq \ell^{(i)}<\infty$,

(1) $m_{\ell+1}^{(i)}(\nu)>0$ and $\widetilde{f}_{i}$ creates a singular string, or $\ell+1=\ell^{(i)}$ and $\widetilde{f}_{i}$ creates a nonsingular string,

(2) $m_{\ell+1}^{(i)}(\nu)=0$ and $\widetilde{f}_{i}$ creates a singular string,

(3) $\ell+1<\ell^{(i)}$ and $\widetilde{f}_{i}$ creates a non-singular string.

D $\ell^{(i)}=\ell$,

(1) $\ell=\ell^{(i)}<\ell_{(i)}$ and $m_{\ell}^{(i)}(\nu)>1$,

(2) $\ell=\ell^{(i)}=\ell_{(i)}$ and $m_{\ell}^{(i)}(\nu)>2$,

(3) $\ell=\ell^{(i)}<\ell_{(i)}$ and $m_{\ell}^{(i)}(\nu)=1$,

(4) $\ell=\ell^{(i)}=\ell_{(i)}$ and $m_{\ell}^{(i)}(\nu)=2$.

$\mathrm{E} \ell^{(i)}<\ell$,

(1) $\ell^{(i)}<\ell$ and $\ell+1<\ell_{(i+1)}$,

(2) $\ell^{(i)}<\ell$ and $\ell_{(i+1)} \leq \ell+1<\ell_{(i)}=\infty$,

(3) $\ell^{(i)}<\ell$ and $\ell_{(i+1)} \leq \ell+1 \leq \ell_{(i)}<\infty$,

(4) $\ell^{(i)}<\ell$ and $\ell_{(i)}=\ell$,

(5) $\ell^{(i)}<\ell$ and $\ell_{(i)}<\ell$.

Finally, let us prepare the following lemma which gives an useful criterion.

Lemma 5.2. Suppose that $\tilde{f}_{i}$ acts on a string $\left(\ell, x_{\ell}\right)$ of $(\nu, J)^{(i)}$ and creates the new string $\left(\ell+1, x_{\ell}-1\right)$.

(1) The string $\left(\ell+1, x_{\ell}-1\right)$ is singular if and only if $P_{\ell+1}^{(i)}(\nu)=x_{\ell}+1$.

(2) Suppose that $\tilde{f}_{i}$ creates a singular string. If there is a string $\left(l, x_{l}\right)$ of $(\nu, J)^{(i)}$ that satisfies $\ell<l$, then we have $P_{\ell+1}^{(i)}(\nu) \leq x_{l}$.

Proof. (1) Suppose that the string $\left(\ell+1, x_{\ell}-1\right)$ is singular. Then it satisfies $P_{\ell+1}^{(i)}(\widetilde{\nu})=x_{\ell}-1$. Since $\tilde{f}_{i}$ adds the box to the $(\ell+1)$-th column of $\nu^{(i)}$, we have $P_{\ell+1}^{(i)}(\widetilde{\nu})=P_{\ell+1}^{(i)}(\nu)-2$. Thus we have $P_{\ell+1}^{(i)}(\nu)=P_{\ell+1}^{(i)}(\widetilde{\nu})+2=x_{\ell}+1$. We can reverse the arguments to obtain the if part.

(2) If $x_{\ell} \geq x_{l}$, then $\tilde{f}_{i}$ will act on $\left(l, x_{l}\right)$ instead of $\left(\ell, x_{\ell}\right)$ since $\ell<l$. Thus we have $x_{\ell}<x_{l}$. Then we have $P_{\ell+1}^{(i)}(\nu)=x_{\ell}+1 \leq x_{l}$. 


\subsection{Proof for Case A}

The proof of this case depends on the following fundamental properties.

Proposition 5.3. If $\ell<\ell^{(i-1)}-1$, we have

$$
P_{\ell^{(i)}-1}^{(i)}(\bar{\nu})>x_{\ell}, \quad P_{\ell_{(i)}-1}^{(i)}(\bar{\nu})>x_{\ell} .
$$

Proof. The proof proceeds by case by case analysis according to the classification (I) to (VII) in Lemma 5.1. We remark that the condition $\ell<\ell^{(i-1)}-1$ automatically implies that $\ell<\ell_{(i+1)}-1$.

Case (I). We have to show $P_{\ell^{(i)}-1}^{(i)}(\nu)>x_{\ell}$ by Lemma 5.1. Suppose if possible that $P_{\ell^{(i)}-1}^{(i)}(\nu) \leq x_{\ell}$. We have $P_{\ell^{(i)}}^{(i)}(\nu) \geq x_{\ell^{(i)}}>x_{\ell}$ since the string $\left(\ell^{(i)}, x_{\ell^{(i)}}\right)$ is not selected by $\tilde{f}_{i}$ whereas $\ell<\ell^{(i)}$. Let $\left(j, x_{j}\right)$ be the longest string of $\nu^{(i)}$ that is strictly shorter than $\ell^{(i)}$. Since $m_{\ell}^{(i)}(\nu)>0$ we have $\ell \leq j$. If $j=\ell^{(i)}-1$, we have $\ell<j\left(\right.$ since $\left.\ell<\ell^{(i-1)}-1 \leq \ell^{(i)}-1\right)$ and $x_{j} \leq P_{j}^{(i)}(\nu) \leq x_{\ell}$, which contradict the definition of $\left(\ell, x_{\ell}\right)$. If $j<\ell^{(i)}-1$, combining $P_{\ell^{(i)}}^{(i)}(\nu)>x_{\ell}$ with the assumption $P_{\ell^{(i)}-1}^{(i)}(\nu) \leq x_{\ell}$, we have $P_{\ell^{(i)}-1}^{(i)}(\nu)<P_{\ell^{(i)}}^{(i)}(\nu)$. Then by the convexity of $P_{k}^{(i)}(\nu)$ between $j \leq k \leq \ell^{(i)}$, we have $x_{j} \leq P_{j}^{(i)}(\nu)<P_{\ell^{(i)}-1}^{(i)}(\nu) \leq x_{\ell}$, which contradicts the minimality of $x_{\ell}$. Thus we have proved $P_{\ell^{(i)}-1}^{(i)}(\nu)>x_{\ell}$.

Case (II). We have to show $P_{\ell^{(i)}-1}^{(i)}(\nu)>x_{\ell}+1$ by Lemma 5.1. Suppose if possible that we have $P_{\ell^{(i)}-1}^{(i)}(\nu) \leq x_{\ell}+1$. Recall that we have $x_{\ell}<x_{\ell^{(i)}} \leq P_{\ell^{(i)}}^{(i)}(\nu)$ by the minimality of $x_{\ell}$ and $\ell<\ell^{(i)}$. Thus we have

$$
P_{\ell^{(i)}-1}^{(i)}(\nu) \leq P_{\ell^{(i)}}^{(i)}(\nu) .
$$

Let $j$ be the length of the longest string of $\nu^{(i)}$ under the condition $j<\ell^{(i)}$. Since $m_{\ell}^{(i)}(\nu)>0$ we have $\ell \leq j$.

Let us show that $j<\ell^{(i-1)}$. Suppose if possible that $\ell^{(i-1)} \leq j$. In particular, we have $\ell<j$ by $\ell<\ell^{(i-1)}-1$. Then we shall show that $P_{j}^{(i)}(\nu) \leq x_{\ell}+1$.

(i) The case $j=\ell^{(i)}-1$. Then $P_{j}^{(i)}(\nu) \leq x_{\ell}+1$ follows from the assumption.

(ii) The case $j<\ell^{(i)}-1$. We can apply the convexity relation of $P_{k}^{(i)}(\nu)$ between $j \leq k \leq \ell^{(i)}$ with (5.1) to deduce that $P_{j}^{(i)}(\nu) \leq P_{\ell^{(i)}-1}^{(i)}(\nu) \leq x_{\ell}+1$.

On the other hand, since $\ell<j$, we have the condition $x_{\ell}<x_{j} \leq P_{j}^{(i)}(\nu)$. Combining this with the above result $P_{j}^{(i)}(\nu) \leq x_{\ell}+1$ we deduce that $P_{j}^{(i)}(\nu)=x_{\ell}+1$ and thus $x_{j}=P_{j}^{(i)}(\nu)$. But then the string $\left(j, x_{j}\right)$ is the singular string whose length satisfies $\ell^{(i-1)} \leq j<\ell^{(i)}$, which is in contradiction to the definition of $\ell^{(i)}$. Hence we have proved $j<\ell^{(i-1)}$.

Then we can apply the convexity relation of $P_{k}^{(i)}(\nu)$ between $\ell^{(i-1)} \leq k \leq \ell^{(i)}$ with (5.1) to deduce that $P_{\ell^{(i-1)}}^{(i)}(\nu) \leq P_{\ell^{(i)}-1}^{(i)}(\nu)$. Since $m_{\ell^{(i-1)}}^{(i-1)}(\nu)>0$ the convexity relation of $P_{k}^{(i)}(\nu)$ between $\ell^{(i-1)}-1 \leq k \leq \ell^{(i-1)}+1$ is strict. Thus we have

$$
P_{j}^{(i)}(\nu)<P_{\ell^{(i-1)}}^{(i)}(\nu) \leq P_{\ell^{(i)}-1}^{(i)}(\nu) \leq x_{\ell}+1,
$$

in particular, $x_{j} \leq P_{j}^{(i)}(\nu) \leq x_{\ell}$. If $\ell<j$, this contradicts the requirement $x_{\ell}<x_{j}$. Hence we are left with the case $j=\ell$. Since we are assuming $\ell<\ell^{(i-1)}-1$ we can refine the above relation as

$$
P_{\ell}^{(i)}(\nu)<P_{\ell^{(i-1)}-1}^{(i)}(\nu)<P_{\ell^{(i-1)}}^{(i)}(\nu) \leq P_{\ell^{(i)}-1}^{(i)}(\nu) \leq x_{\ell}+1,
$$


that is, $P_{\ell}^{(i)}(\nu)<x_{\ell}$. But this contradicts the requirement that the string $\left(\ell, x_{\ell}\right)$ must satisfy $x_{\ell} \leq P_{\ell}^{(i)}(\nu)$. Thus we have proved $P_{\ell^{(i)}-1}^{(i)}(\nu)>x_{\ell}+1$.

Case (III). We have to show $P_{\ell_{(i)}-1}^{(i)}(\nu)>x_{\ell}$ by Lemma 5.1. Since $\ell_{(i)}=\ell^{(i)}$ we can use the same arguments of (I) to obtain the result.

Case $(I V)$. We have to show $P_{\ell_{(i)}-1}^{(i)}(\nu)>x_{\ell}+1$ by Lemma 5.1. Since $\ell_{(i)}=\ell^{(i)}$ we can use the same arguments of (II) to obtain the result.

Case (V). We have to show $P_{\ell_{(i)}-1}^{(i)}(\nu) \geq x_{\ell}$ by Lemma 5.1. Suppose if possible that $P_{\ell_{(i)}-1}^{(i)}(\nu)<x_{\ell}$. Let $j$ be the length of the longest string of $\nu^{(i)}$ under the condition $j<\ell_{(i)}$. Since $m_{\ell^{(i)}}^{(i)}(\nu)>0$ we have $\ell^{(i)} \leq j$. If $j=\ell_{(i)}-1$ we have $x_{j} \leq P_{j}^{(i)}(\nu)<x_{\ell}$ which is in contradiction to the minimality of $x_{\ell}$. Thus assume that $j<\ell_{(i)}-1$. Then by the convexity relation of $P_{k}^{(i)}(\nu)$ between $j \leq k \leq \ell_{(i)}$ we have $P_{\ell_{(i)}-1}^{(i)}(\nu) \geq \min \left\{P_{j}^{(i)}(\nu), P_{\ell_{(i)}}^{(i)}(\nu)\right\}$. However this implies that $x_{\ell}>x_{j}$ or $x_{\ell}>x_{\ell_{(i)}}$ both of which are in contradiction to the minimality of $x_{\ell}$. Thus we conclude that $P_{\ell_{(i)}-1}^{(i)}(\nu) \geq x_{\ell}$.

Case (VI). We have to show $P_{\ell_{(i)}-1}^{(i)}(\nu)>x_{\ell}$ by Lemma 5.1. Suppose if possible that $P_{\ell_{(i)}-1}^{(i)}(\nu) \leq x_{\ell}$. Let $j$ be the length of the longest string of $\nu^{(i)}$ under the condition $j<\ell_{(i)}$. Since $m_{\ell^{(i)}}^{(i)}(\nu)>0$ we have $\ell^{(i)} \leq j$. In particular, we have $\ell<j$ by $\ell<\ell^{(i-1)}-1$. If $j=\ell_{(i)}-1$ we have $x_{j} \leq x_{\ell}$ which contradicts the relation $x_{j}>x_{\ell}$ required by $\ell<j$. So suppose that $j<\ell_{(i)}-1$. Again we have $P_{\ell_{(i)}-1}^{(i)}(\nu) \geq \min \left\{P_{j}^{(i)}(\nu), P_{\ell_{(i)}}^{(i)}(\nu)\right\}$. Then we have $x_{\ell} \geq x_{j}$ or $x_{\ell} \geq x_{\ell_{(i)}}$. This is a contradiction since $\ell<j$ and $\ell<\ell^{(i)}$.

Case (VII). We have to show $P_{\ell_{(i)}-1}^{(i)}(\nu)>x_{\ell}+1$ by Lemma 5.1. We can prove this by the same arguments of the case (II) if we replace $\ell^{(i)}$ and $\ell^{(i-1)}$ there by $\ell_{(i)}$ and $\ell_{(i+1)}$ respectively.

Proposition 5.4. If $\ell<\ell^{(i-1)}-1$, we have the following identities:
$\overline{\widetilde{\nu}}=\widetilde{\bar{\nu}}$
(2) $\overline{\widetilde{J}}=\widetilde{\bar{J}}$.

Proof. (1) Step 1. Let us consider the case $\ell^{(i-1)}=\infty$. Then $\delta$ will not choose any string from $(\nu, J)^{(i-1)}$ so that $\widetilde{f}_{i}$ chooses the same string before and after $\delta$. Next, recall that $\widetilde{f}_{i}$ does not change coriggings of untouched strings. Then we have $\widetilde{\ell}^{(a)}=\ell^{(a)}$ for all $a \leq i-1$. In particular, we have $\widetilde{\ell}^{(i-1)}=\infty$. Thus we have $\widetilde{\ell}^{(a)}=\ell^{(a)}=\infty$ for all $a \geq i-1$.

Step 2. Based on the above observation, let us assume that $\ell^{(i-1)}<\infty$ in the sequel. Let us show that $\delta$ chooses the same strings before and after $\widetilde{f}_{i}$. Again we have $\widetilde{\ell}^{(a)}=\ell^{(a)}$ for all $a \leq i-1$ since $\tilde{f}_{i}$ does not change coriggings of untouched strings. Recall that we have $\ell+1<\ell^{(i-1)}=\widetilde{\ell}^{(i-1)}$ by the assumption. Thus $\delta$ cannot choose the string $\left(\ell+1, x_{\ell}-1\right)$ created by $\widetilde{f}_{i}$. Thus we have $\widetilde{\ell}^{(i)}=\ell^{(i)}$ which implies that $\widetilde{\ell}^{(a)}=\ell^{(a)}$ for all $a \geq i$ and $\widetilde{\ell}_{(a)}=\ell_{(a)}$ for all $a$.

Step 3. Let us show that $\widetilde{f}_{i}$ chooses the same string before and after $\delta$. Recall that $\delta$ creates the strings $\left(\ell^{(i)}-1, P_{\ell^{(i)}-1}^{(i)}(\bar{\nu})\right)$ and $\left(\ell_{(i)}-1, P_{\ell_{(i)}-1}^{(i)}(\bar{\nu})\right)$ of $(\bar{\nu}, \bar{J})^{(i)}$. Note that the string $\left(\ell, x_{\ell}\right)$ remains as it is in $(\bar{\nu}, \bar{J})^{(i)}$ since $\delta$ acting on $(\nu, J)$ does not touch the string by the assumption $\ell<\ell^{(i-1)}$. By Proposition 5.3 we have $P_{\ell^{(i)}-1}^{(i)}(\bar{\nu})>x_{\ell}$ and $P_{\ell_{(i)}-1}^{(i)}(\bar{\nu})>x_{\ell}$. Therefore $\widetilde{f}_{i}$ acts on the same string $\left(\ell, x_{\ell}\right)$ before and after the application of $\delta$.

To summarize, we have $\overline{\widetilde{\nu}}=\widetilde{\bar{\nu}}$.

(2) It is enough to consider the strings $\left(\ell^{(a)}, P_{\ell^{(a)}}^{(a)}(\nu)\right)$ of $(\nu, J)^{(a)}$ for $a=i-1, i, i+1$, since $\tilde{f}_{i}$ adds a box to the same place before and after $\delta$ and since $\delta$ does not change riggings 
for untouched strings. For example, the string for $a=i-1$ case behaves as follows:

$$
\begin{array}{llll}
\left(\ell^{(i-1)}, P_{\ell^{(i-1)}}^{(i-1)}(\nu)\right) & \stackrel{\widetilde{f}_{i}}{\longmapsto}\left(\ell^{(i-1)}, P_{\ell^{(i-1)}}^{(i-1)}(\widetilde{\nu})\right) & \stackrel{\delta}{\longmapsto} & \left(\ell^{(i-1)}-1, P_{\ell^{(i-1)}-1}^{(i-1)}(\overline{\widetilde{\nu}})\right), \\
\left(\ell^{(i-1)}, P_{\ell^{(i-1)}}^{(i-1)}(\nu)\right) & \stackrel{\delta}{\longmapsto}\left(\ell^{(i-1)}-1, P_{\ell^{(i-1)}-1}^{(i)}(\bar{\nu})\right) & \stackrel{\widetilde{f}_{i}}{\longmapsto}\left(\ell^{(i-1)}-1, P_{\ell^{(i-1)}-1}^{(i)}(\widetilde{\bar{\nu}})\right) .
\end{array}
$$

In all cases, the riggings are determined so that $\widetilde{f}_{i}$ and $\delta$ create the singular strings. Since we have $\overline{\widetilde{\nu}}=\widetilde{\bar{\nu}}$, we obtain $P_{\ell^{(i-1)}-1}^{(i-1)}(\overline{\widetilde{\nu}})=P_{\ell^{(i-1)}-1}^{(i)}(\widetilde{\bar{\nu}})$. The other cases are similar. Thus we conclude that $\overline{\widetilde{J}}=\widetilde{\bar{J}}$.

\subsection{Proof for Case B}

Let us show the commutativity of $\widetilde{f}_{i}$ and $\delta$ for the Case B, that is, $\ell^{(i-1)} \leq \ell+1<\ell^{(i)}=\infty$. We divide the proof into the two cases.

\subsubsection{Case 1: $\tilde{f}_{i}$ creates a non-singular string}

Proposition 5.5. Suppose that $\ell^{(i-1)} \leq \ell+1<\ell^{(i)}=\infty$. If $\tilde{f}_{i}$ acting on $(\nu, J)$ creates a non-singular string, then we have the following identities:

$$
\text { (1) } \quad \overline{\widetilde{\nu}}=\widetilde{\bar{\nu}}, \quad(2) \quad \overline{\widetilde{J}}=\widetilde{\bar{J}}
$$

Proof. (1) Since $\widetilde{f}_{i}$ does not change coriggings, $\delta$ chooses the same strings in $(\nu, J)^{(a)}$ and $(\widetilde{\nu}, \widetilde{J})^{(a)}$ for all $a<i$. Also, since $\widetilde{f}_{i}$ creates a non-singular string, $\delta$ does not choose a string from $(\widetilde{\nu}, \widetilde{J})^{(i)}$, which coincides with the action of $\delta$ on $(\nu, J)^{(i)}$. Next, since $\delta$ does not touch $(\nu, J)^{(i)}$, we have $\bar{J}^{(i)}=J^{(i)}$. Thus $\widetilde{f}_{i}$ chooses the same string in both $(\nu, J)^{(i)}$ and $(\bar{\nu}, \bar{J})^{(i)}$. To summarize, $\delta$ and $\tilde{f}_{i}$ choose the same strings in $\delta \circ \widetilde{f}_{i}$ and $\widetilde{f}_{i} \circ \delta$ which implies $\overline{\widetilde{\nu}}=\widetilde{\widetilde{\nu}}$.

(2) We only need to consider $J^{(i-1)}$ and $J^{(i)}$ in this case. If the string is not touched by $\delta$ and $\widetilde{f}_{i}$, the riggings after $\delta \circ \widetilde{f}_{i}$ and $\widetilde{f}_{i} \circ \delta$ coincide since $\delta$ and $\widetilde{f}_{i}$ choose the same strings in both cases respectively. The string $\left(\ell^{(i-1)}, P_{\ell^{(i-1)}}^{(i-1)}(\nu)\right)$ becomes $\left(\ell^{(i-1)}-1, P_{\ell^{(i-1)}-1}^{(i-1)}(\overline{\widetilde{\nu}})\right)$ and $\left(\ell^{(i-1)}-1, P_{\ell(i-1)-1}^{(i-1)}(\widetilde{\bar{\nu}})\right)$ after application of $\delta \circ \widetilde{f}_{i}$ and $\widetilde{f}_{i} \circ \delta$ respectively. Since $\overline{\widetilde{\nu}}=\widetilde{\bar{\nu}}$ we see the coincidence of the riggings. As for the string $\left(\ell, x_{\ell}\right)$, we see that both $\delta \circ \widetilde{f}_{i}$ and $\widetilde{f}_{i} \circ \delta$ give the string $\left(\ell+1, x_{\ell}-1\right)$. Hence we have $\overline{\widetilde{J}}=\widetilde{\bar{J}}$.

\subsubsection{Case 2: $\widetilde{f}_{i}$ creates a singular string}

This situation does not satisfy the condition of Proposition 4.7 due to the following fact.

Proposition 5.6. Suppose that $\ell^{(i-1)} \leq \ell+1<\ell^{(i)}=\infty$. If $\widetilde{f}_{i}$ acting on $(\nu, J)$ creates a singular string, then we have

$$
\tilde{f}_{i} \circ \delta(\nu, J)=0
$$

Proof. Since $\ell^{(i)}=\infty$, we have $(\nu, J)^{(i)}=(\bar{\nu}, \bar{J})^{(i)}$ and thus $\bar{\ell}=\ell$. Then the string $\left(\ell, x_{\ell}\right)$ in $(\nu, J)^{(i)}$ will become $\left(\ell+1, x_{\ell}-1\right)$ in $\widetilde{f}_{i} \circ \delta(\nu, J)$. Let us compute $P_{\ell+1}^{(i)}(\widetilde{\bar{\nu}})$. From Lemma 5.2(1), we have $P_{\ell+1}^{(i)}(\nu)=x_{\ell}+1$. By the assumption $\ell^{(i-1)} \leq \ell+1<\ell^{(i)}$ we have $P_{\ell+1}^{(i)}(\bar{\nu})=P_{\ell+1}^{(i)}(\nu)-1=x_{\ell}$. Therefore we have $P_{\ell+1}^{(i)}(\widetilde{\bar{\nu}})=P_{\ell+1}^{(i)}(\bar{\nu})-2=x_{\ell}-2$. But then the string $\left(\ell+1, x_{\ell}-1\right)$ in $\tilde{f}_{i} \circ \delta(\nu, J)$ satisfies $P_{\ell+1}^{(i)}(\widetilde{\bar{\nu}})<x_{\ell}-1$ which implies that $\tilde{f}_{i} \circ \delta(\nu, J)$ is not a valid rigged configuration. Thus we have $\tilde{f}_{i} \circ \delta(\nu, J)=0$. 
Example 5.7. Consider the following rigged configuration $(\nu, J)$ of type $\left(B^{1,1}\right)^{\otimes 4} \otimes B^{1,3} \otimes B^{2,1} \otimes$ $B^{2,2} \otimes B^{3,1}$ of $D_{5}^{(1)}$
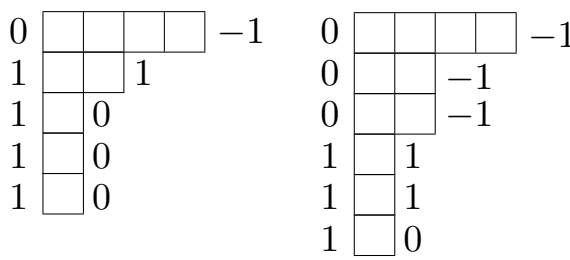

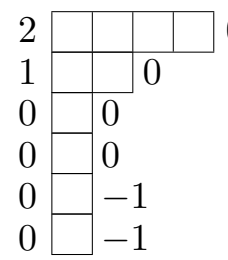

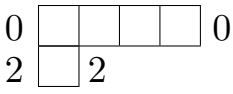

The corresponding tensor product $\Phi(\nu, J)$ is

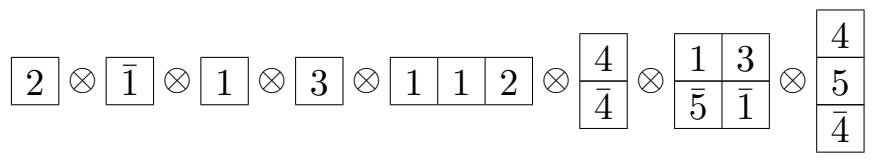

$\widetilde{f}_{2}$ acts on the string $(4,-1)$ of $(\nu, J)^{(2)}$ and makes it into $(5,-2)$ of $(\widetilde{\nu}, \widetilde{J})^{(2)}$. Since we have $P_{5}^{(2)}(\widetilde{\nu})=-2$, the latter string is singular. In particular, we see that $\widetilde{f}_{2}(\nu, J) \neq 0$. Now $(\bar{\nu}, \bar{J})$ is

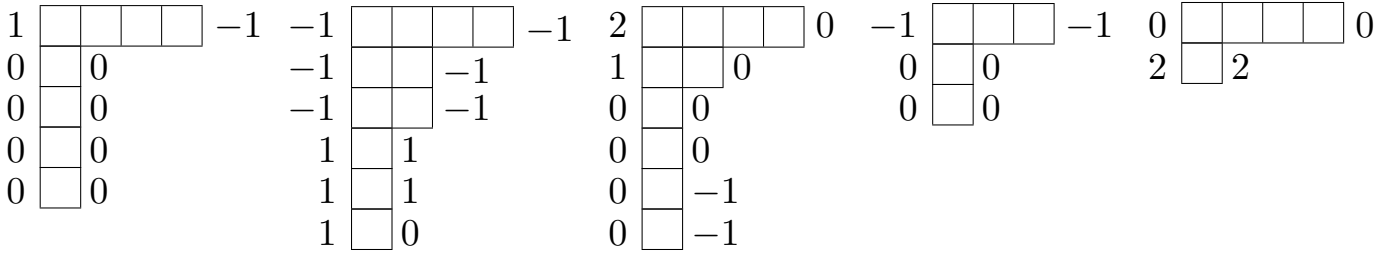

Thus we have $\ell^{(1)}=2<\ell+1=5<\ell^{(2)}=\infty$ (see Proposition 5.6). Indeed, we have $P_{5}^{(2)}(\widetilde{\bar{\nu}})=-3$ and thus $\widetilde{f}_{2}(\bar{\nu}, \bar{J})=0$.

\subsection{Proof for Case C: preliminary steps}

\subsubsection{Classification}

Recall that the defining condition of the Case $\mathrm{C}$ is $\ell^{(i-1)} \leq \ell+1 \leq \ell^{(i)}<\infty$. We divide the proof into the three cases according to the following extra conditions:

(1) $m_{\ell+1}^{(i)}(\nu)>0$ and $\tilde{f}_{i}$ creates a singular string, or

$\ell+1=\ell^{(i)}$ and $\tilde{f}_{i}$ creates a non-singular string,

(2) $m_{\ell+1}^{(i)}(\nu)=0$ and $\widetilde{f}_{i}$ creates a singular string,

(3) $\ell+1<\ell^{(i)}$ and $\tilde{f}_{i}$ creates a non-singular string.

We give several remarks on the classification. In case $(2)$, the condition $m_{\ell+1}^{(i)}(\nu)=0$ automatically implies that $\ell+1<\ell^{(i)}$ by the assumption $\ell+1 \leq \ell^{(i)}$. On the other hand, in the first case of case $(1)$, we will show that $\ell+1=\ell^{(i)}$. We remark that we may have $m_{\ell+1}^{(i)}(\nu)>0$ in case (3).

\subsubsection{A common property for Case C}

In this case, we can show the following property. Note that the corresponding analysis for the case $\ell^{(i)}=\ell_{(i)}$ is included in the analysis of $P_{\ell^{(i)}-1}^{(i)}(\bar{\nu})$ which will be given later in this section.

Proposition 5.8. Suppose that $\ell+1 \leq \ell^{(i)}<\ell_{(i)}<\infty$. Then we have

$$
P_{\ell_{(i)}-1}^{(i)}(\bar{\nu})>x_{\ell}
$$


Proof. We follow the classification in Lemma 5.1. Since $\ell^{(i)}<\ell_{(i)}$, we have to consider Cases (V), (VI) and (VII). Let $j$ be the largest integer satisfying $j<\ell_{(i)}$ and $m_{j}^{(i)}(\nu)>0$. From $\ell^{(i)}<\ell_{(i)}$, we see that $\ell^{(i)} \leq j$ and in particular we have $\ell<j$. In fact we only need the latter relation $\ell<j$ in the following proof ${ }^{4}$.

Case (V). According to Lemma 5.1 we have to show $P_{\ell_{(i)}-1}^{(i)}(\nu) \geq x_{\ell}$ under the condition $\ell^{(i)}<\ell^{(i+1)}=\cdots=\ell_{(i)}$. Suppose if possible that $P_{\ell_{(i)}-1}^{(i)}(\nu)<x_{\ell}$. If $j=\ell_{(i)}-1$, then we have $x_{\ell_{(i)}-1} \leq P_{\ell_{(i)}-1}^{(i)}(\nu)<x_{\ell}$ which contradicts the minimality of $x_{\ell}$. So suppose that $j<\ell_{(i)}-1$. By the assumption we see that $\ell<\ell_{(i)}$. Then we have $P_{\ell_{(i)}}^{(i)}(\nu) \geq x_{\ell_{(i)}}>x_{\ell}$. Thus we have $P_{\ell_{(i)}-1}^{(i)}(\nu)<P_{\ell_{(i)}}^{(i)}(\nu)$. By the convexity of $P_{k}^{(i)}(\nu)$ between $j \leq k \leq \ell_{(i)}$, we have $P_{j}^{(i)}(\nu)<P_{\ell_{(i)}-1}^{(i)}(\nu)<x_{\ell}$. Since $x_{j} \leq P_{j}^{(i)}(\nu)$, this contradicts the minimality of $x_{\ell}$. Thus we conclude that $P_{\ell_{(i)}-1}^{(i)}(\nu) \geq x_{\ell}$.

Case (VI). According to Lemma 5.1 we have to show $P_{\ell_{(i)}-1}^{(i)}(\nu)>x_{\ell}$ under the condition $\ell^{(i+1)}<\ell_{(i+1)}=\ell_{(i)}$. Suppose if possible that $P_{\ell_{(i)}-1}^{(i)}(\nu) \leq x_{\ell}$. If $j=\ell_{(i)}-1$, then we have $x_{j} \leq P_{j}^{(i)}(\nu) \leq x_{\ell}$. However this relation contradicts the requirement $x_{j}>x_{\ell}$ which follows from $\ell<j$. Next, suppose that $j<\ell_{(i)}-1$. Then, as in the Case $(\mathrm{V})$, we have $P_{\ell_{(i)}}^{(i)}(\nu)>x_{\ell}$ by $\ell<\ell_{(i)}$. Thus we have $P_{\ell_{(i)}-1}^{(i)}(\nu)<P_{\ell_{(i)}}^{(i)}(\nu)$. By the similar arguments in the previous case, we can deduce the contradiction. Therefore we obtain $P_{\ell_{(i)}-1}^{(i)}(\nu)>x_{\ell}$ in this case.

Case (VII). According to Lemma 5.1 we have to show $P_{\ell_{(i)}-1}^{(i)}(\nu)>x_{\ell}+1$ under the condition $\ell_{(i+1)}<\ell_{(i)}$. Suppose if possible that $P_{\ell_{(i)}-1}^{(i)}(\nu) \leq x_{\ell}+1$. If $j=\ell_{(i)}-1$, then we have $P_{j}^{(i)}(\nu) \geq x_{j}>x_{\ell}$ by $j>\ell$. Then the only possibility that is compatible with the assumption is $P_{j}^{(i)}(\nu)=x_{j}=x_{\ell}+1$. In particular, the string $\left(j, x_{j}\right)$ is singular whose length satisfies $\ell_{(i+1)} \leq j<\ell_{(i)}$. However this is in contradiction to the definition of $\ell_{(i)}$.

If $j<\ell_{(i)}-1$, again we have $P_{\ell_{(i)}}^{(i)}(\nu)>x_{\ell}$ as in the Case $(\mathrm{V})$. Similarly, by $\ell<j$ we have $P_{j}^{(i)}(\nu)>x_{\ell}$. Then by the convexity of $P_{k}^{(i)}(\nu)$ between $j \leq k \leq \ell_{(i)}$, the only possibility that is compatible with $P_{\ell_{(i)}-1}^{(i)}(\nu) \leq x_{\ell}+1$ is $P_{j}^{(i)}(\nu)=\cdots=P_{\ell_{(i)}}^{(i)}(\nu)=x_{\ell}+1$. Since $P_{j}^{(i)}(\nu) \geq x_{j}>x_{\ell}$ it has to be $x_{j}=x_{\ell}+1$, in particular, the string $\left(j, x_{j}\right)$ is singular. If $\ell_{(i+1)} \leq j$, this contradicts the definition of $\ell_{(i)}$. On the other hand, if $j<\ell_{(i+1)}, m_{\ell_{(i+1)}}^{(i+1)}(\nu)>0$ means that the convexity of $P_{k}^{(i)}(\nu)$ between $\ell_{(i+1)}-1 \leq k \leq \ell_{(i+1)}+1$ is strict. This is in contradiction to the relation $P_{j}^{(i)}(\nu)=\cdots=P_{\ell_{(i)}}^{(i)}(\nu)=x_{\ell}+1$.

\subsection{Proof for Case C (1)}

Proposition 5.9. Suppose that $\ell^{(i-1)} \leq \ell+1 \leq \ell^{(i)}<\infty, m_{\ell+1}^{(i)}(\nu)>0$ and $\tilde{f}_{i}$ creates a singular string. Then we have the following identities:
(1) $\overline{\widetilde{\nu}}=\widetilde{\bar{\nu}}$,
(2) $\quad \overline{\widetilde{J}}=\widetilde{\bar{J}}$.

Proof. (1) To begin with let us show that $\ell+1=\ell^{(i)}$. By the assumption $m_{\ell+1}^{(i)}(\nu)>0$ there exists the string $\left(\ell+1, x_{\ell+1}\right)$ of $(\nu, J)^{(i)}$. We show that the string $\left(\ell+1, x_{\ell+1}\right)$ is singular. For this we derive the following three relations:

\footnotetext{
${ }^{4}$ We will use this property in the proof of Proposition 5.24.
} 
- $P_{\ell+1}^{(i)}(\nu)=x_{\ell}+1$ by Lemma 5.2,

- $x_{\ell}+1 \leq x_{\ell+1}$, that is, $x_{\ell}<x_{\ell+1}$, since the string $\left(\ell+1, x_{\ell+1}\right)$ of $(\nu, J)$ is not selected by $\tilde{f}_{i}$ although it is longer than $\ell$,

- $x_{\ell+1} \leq P_{\ell+1}^{(i)}(\nu)$ by definition of the rigged configurations.

Combining these three inequalities, we obtain $P_{\ell+1}^{(i)}(\nu)=x_{\ell+1}$ and thus the string $\left(\ell+1, x_{\ell+1}\right)$ is singular. Suppose if possible that $\ell+1<\ell^{(i)}$. Then the string $\left(\ell+1, x_{\ell+1}\right)$ is singular whose length satisfies $\ell^{(i-1)} \leq \ell+1$, contradicting the definition of $\ell^{(i)}$. Thus $\ell+1=\ell^{(i)}$. Then we can show the commutativity of $\widetilde{f}_{i}$ and $\delta$ as follows.

Let us consider the case $\delta \circ \widetilde{f}_{i}$. It is enough to analyze the strings $\left(\ell, x_{\ell}\right)$ and $\left(\ell+1, P_{\ell+1}^{(i)}(\nu)\right)$ in $(\nu, J)^{(i)}$ :

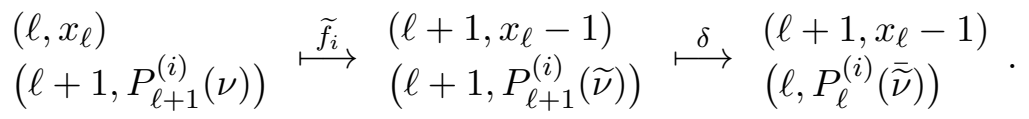

Recall that $\tilde{f}_{i}$ does not change coriggings other than the touched string. In particular, we have $\widetilde{\ell}^{(i-1)}=\ell^{(i-1)}$. Thus the next $\delta$ can act on one of the length $\ell+1$ singular strings.

Let us consider the case $\tilde{f}_{i} \circ \delta$. Recall that if $\ell^{(i)}<\ell_{(i)}$ we have $P_{\ell_{(i)}-1}^{(i)}(\bar{\nu})>x_{\ell}$ by Proposition 5.8. Therefore $\tilde{f}_{i}$ will not act on the length $\ell_{(i)}-1$ string of $(\bar{\nu}, \bar{J})^{(i)}$ that has been created by $\delta$. On the other hand, if $\ell^{(i)}=\ell_{(i)}$ we do not need to take care of this issue. Thus it is enough to analyze the behaviors of the lengths $\ell$ and $\ell+1$ strings:

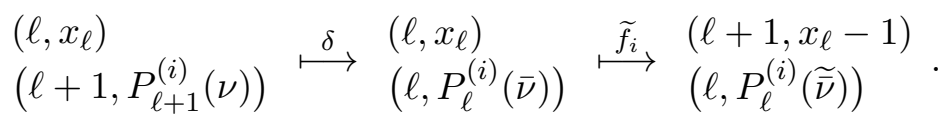

Recall that $\ell+1=\ell^{(i)}$ so that $\delta$ acts on the string $\left(\ell+1, P_{\ell+1}^{(i)}(\nu)\right)$. Also, since $(\bar{\nu}, \bar{J})$ is the valid rigged configuration, we have the requirement $P_{\ell}^{(i)}(\bar{\nu}) \geq x_{\ell}$. Thus the string $\left(\ell, x_{\ell}\right)$ has the largest length among the strings of the smallest rigging within $(\bar{\nu}, \bar{J})^{(i)}$.

To summarize, we are left with lengths $\ell$ and $\ell+1$ strings in both $\delta \circ \widetilde{f}_{i}$ and $\widetilde{f}_{i} \circ \delta$. Thus we have $\overline{\widetilde{\nu}}=\widetilde{\bar{\nu}}$.

(2) We have $P_{\ell}^{(i)}(\overline{\widetilde{\nu}})=P_{\ell}^{(i)}(\widetilde{\bar{\nu}})$ by $\overline{\widetilde{\nu}}=\widetilde{\bar{\nu}}$. Thus $\overline{\widetilde{J}}=\widetilde{\bar{J}}$.

Proposition 5.10. Suppose that we have $\ell+1=\ell^{(i)}$ and $\tilde{f}_{i}$ acting on $(\nu, J)$ creates the nonsingular string. Then we have the following identities:

$$
\text { (1) } \quad \overline{\widetilde{\nu}}=\widetilde{\bar{\nu}}, \quad(2) \quad \overline{\widetilde{J}}=\widetilde{\bar{J}} .
$$

Proof. Since $\ell+1=\ell^{(i)}$ there is a singular string $\left(\ell+1, P_{\ell+1}^{(i)}(\nu)\right)$ in $(\nu, J)^{(i)}$. Then this string and $\left(\ell, x_{\ell}\right)$ behave just as in the proof of the previous proposition which confirms the claim.

\subsection{Proof for Case C (2)}

\subsubsection{Outline}

As the second step, we treat the case when $m_{\ell+1}^{(i)}(\nu)=0$ and $\widetilde{f}_{i}$ creates a singular string. In this case, we will show the following assertion by case by case analysis.

Proposition 5.11. Suppose that $\ell^{(i-1)} \leq \ell+1<\ell^{(i)}<\infty$ and $m_{\ell+1}^{(i)}(\nu)=0$. If $\tilde{f}_{i}$ acting on $(\nu, J)$ creates a singular string, then the following two conditions are satisfied:

$$
\begin{aligned}
& P_{\ell^{(i)}-1}^{(i)}(\bar{\nu})=x_{\ell}, \\
& m_{k}^{(i+1)}(\nu)=0 \quad \text { for } \ell<k<\ell^{(i)} .
\end{aligned}
$$


Note that we have $\ell+1 \neq \ell^{(i)}$ by $m_{\ell+1}^{(i)}(\nu)=0$. To begin with, we explain how these properties provide the proof of $\overline{\widetilde{\nu}}=\widetilde{\bar{\nu}}$.

Proposition 5.12. Suppose that $\ell^{(i-1)} \leq \ell+1<\ell^{(i)}<\infty, m_{\ell+1}^{(i)}(\nu)=0$ and $\widetilde{f}_{i}$ acting on $(\nu, J)$ creates a singular string. Then we have $\overline{\widetilde{\nu}}=\widetilde{\bar{\nu}}$.

Proof. Let us analyze $\tilde{f}_{i} \circ \delta$ and $\delta \circ \widetilde{f}_{i}$ respectively.

Step 1. Let us consider the case $\widetilde{f}_{i} \circ \delta$. Let us consider the strings $\left(\ell^{(i)}-1, P_{\ell^{(i)}-1}^{(i)}(\bar{\nu})\right)$ and $\left(\ell_{(i)}-1, P_{\ell_{(i)}-1}^{(i)}(\bar{\nu})\right)$ of $(\bar{\nu}, \bar{J})^{(i)}$ created by $\delta$. By $(5.2)$ we see that the rigging of the string $\left(\ell^{(i)}-1, P_{\ell^{(i)}-1}^{(i)}(\bar{\nu})\right)$ of $(\bar{\nu}, \bar{J})$ is $x_{\ell}$. On the other hand, if $\ell^{(i)}<\ell_{(i)}$ we have $P_{\ell_{(i)}-1}^{(i)}(\bar{\nu})>x_{\ell}$ by Proposition 5.8. Since we are assuming that $\ell<\ell^{(i)}-1$, the string $\left(\ell^{(i)}-1, P_{\ell^{(i)}-1}^{(i)}(\bar{\nu})\right)$ has the largest length among the strings of smallest riggings within $(\bar{\nu}, \bar{J})^{(i)}$. Thus $\tilde{f}_{i}$ adds a box to the length $\ell^{(i)}-1$ string that has been shortened by $\delta$.

Step 2. Let us consider the case $\delta \circ \widetilde{f}_{i}$. Recall that $\widetilde{f}_{i}$ does not change coriggings of untouched strings. By the assumption $\widetilde{f}_{i}$ creates the singular string $\left(\ell+1, x_{\ell}-1\right)$ whose length satisfies $\ell^{(i-1)} \leq \ell+1<\ell^{(i)}$. Hence $\delta$ choose this string and obtain $\tilde{\ell}^{(i)}=\ell+1$. By definition of $\delta$ we have $\widetilde{\ell^{(i)}}=\ell+1 \leq \widetilde{\ell}^{(i+1)}$ and by $(5.3)$ we conclude that $\ell^{(i)} \leq \widetilde{\ell}^{(i+1)}$. Thus $\delta$ choose a string of length $\ell^{(i+1)}$ from $(\widetilde{\nu}, \widetilde{J})^{(i+1)}$. Therefore we obtain $\widetilde{\ell}^{(a)}=\ell^{(a)}$ for all $a \neq i$ and $\tilde{\ell}_{(a)}=\ell_{(a)}$ for all $a$.

Step 3. To summarize, we have $\overline{\widetilde{\nu}}^{(a)}=\widetilde{\bar{\nu}}^{(a)}=\bar{\nu}^{(a)}$ for all $a \neq i$ and $\overline{\widetilde{\nu}}^{(i)}=\widetilde{\bar{\nu}}^{(i)}$ is obtained by removing a box from length $\ell_{(i)}$ string of $\nu^{(i)}$.

The remaining property $\overline{\widetilde{J}}=\widetilde{\bar{J}}$ will be checked from case by case analysis and it will be done along with the proof of Proposition 5.11.

\subsubsection{Classification}

We divide the proofs of Proposition 5.11 as well as the identity $\overline{\widetilde{J}}=\widetilde{\bar{J}}$ into the two cases according to the following classification.

Lemma 5.13. Suppose that $\ell^{(i-1)} \leq \ell+1<\ell^{(i)}<\infty$. Then there are only two possibilities as follows:

$$
\begin{aligned}
& \left(\ell, x_{\ell}\right) \text { is singular and } \ell=\ell^{(i-1)}-1, \\
& \left(\ell, x_{\ell}\right) \text { is non-singular. }
\end{aligned}
$$

Proof. Suppose that the string $\left(\ell, x_{\ell}\right)$ is singular. If its length satisfies $\ell^{(i-1)} \leq \ell<\ell^{(i)}$, then it is in contradiction to the definition of $\ell^{(i)}$. Therefore we must have $\ell=\ell^{(i-1)}-1$.

\subsubsection{Proof for the case (5.4)}

Under the present assumptions, let us show the following property.

Proposition 5.14. Suppose that we have $\ell^{(i-1)}=\ell+1<\ell^{(i)}<\infty, m_{\ell+1}^{(i)}(\nu)=0$, the string $\left(\ell, x_{\ell}\right)$ is singular and $\tilde{f}_{i}$ creates a singular string. Then we have

$$
P_{\ell}^{(i)}(\nu)=x_{\ell}, \quad P_{\ell+1}^{(i)}(\nu)=P_{\ell+2}^{(i)}(\nu)=\cdots=P_{\ell^{(i)}}^{(i)}(\nu)=x_{\ell}+1 .
$$


Proof. Since $\tilde{f}_{i}$ creates a singular string, we have

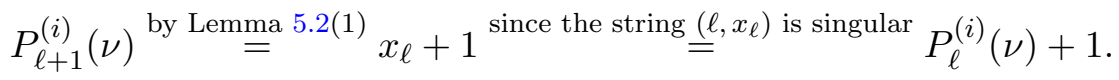

Due to the existence of the length $\ell^{(i-1)}=\ell+1$ string at $\nu^{(i-1)}$ and the assumption $m_{\ell+1}^{(i)}(\nu)=0$, the vacancy number $P_{k}^{(i)}(\nu)$ is strictly convex between the interval $\ell \leq k \leq \ell+2$. Then the relation (5.7) implies $P_{\ell+1}^{(i)}(\nu) \geq P_{\ell+2}^{(i)}(\nu)$. Let us show that this inequality is in fact equality. For this let $j$ be the length of the smallest string of $\nu^{(i)}$ that is longer than $\ell$. Since $m_{\ell+1}^{(i)}(\nu)=0$ we have $\ell^{(i-1)}=\ell+1<j \leq \ell^{(i)}$. By Lemma 5.2(2) we have

$$
P_{\ell+1}^{(i)}(\nu) \leq x_{j} \leq P_{j}^{(i)}(\nu)
$$

Then by the convexity of $P_{k}^{(i)}(\nu)$ between $\ell \leq k \leq j$, the relations $P_{\ell+1}^{(i)}(\nu) \geq P_{\ell+2}^{(i)}(\nu)$ and $P_{\ell+1}^{(i)}(\nu) \leq P_{j}^{(i)}(\nu)$ imply the relation $P_{\ell+1}^{(i)}(\nu)=P_{\ell+2}^{(i)}(\nu)=\cdots=P_{j}^{(i)}(\nu)$. Combining this equality with (5.8) we obtain $x_{j}=P_{j}^{(i)}(\nu)$, thus the string $\left(j, x_{j}\right)$ is singular. By the relation $\ell^{(i-1)}<j \leq \ell^{(i)}$ we deduce that $j=\ell^{(i)}$.

Corollary 5.15. Under the same assumptions of Proposition 5.14, we have

(1) $m_{\ell+1}^{(i-1)}(\nu)=1$,

(2) $m_{k}^{(i-1)}(\nu)=0$ for $\ell+1<k<\ell^{(i)}$,

(3) $m_{k}^{(i)}(\nu)=0$ for $\ell+1 \leq k<\ell^{(i)}$,

(4) $m_{k}^{(i+1)}(\nu)=0$ for $\ell+1 \leq k<\ell^{(i)}$.

Proof. The assertions for $m_{k}^{(i)}(\nu)$ given in (3) are proved at the end of the proof of Proposition 5.14.

Let us show the assertions for $m_{k}^{(i+1)}(\nu)$. If $m_{k}^{(i+1)}(\nu)>0$ for some $\ell+1<k<\ell^{(i)}$, the relation (5.6) must be strictly convex around such $k$, which is a contradiction. Therefore $m_{k}^{(i+1)}(\nu)=0$ for all $\ell+1<k<\ell^{(i)}$. By using (5.6) and the assumption $m_{\ell+1}^{(i)}(\nu)=0$, the estimate of Lemma 3.13 with $l=\ell+1$ gives

$$
\begin{aligned}
& -P_{\ell}^{(i)}(\nu)+2 P_{\ell+1}^{(i)}(\nu)-P_{\ell+2}^{(i)}(\nu)=1 \\
& \quad \geq m_{\ell+1}^{(i-1)}(\nu)-2 m_{\ell+1}^{(i)}(\nu)+m_{\ell+1}^{(i+1)}(\nu)=m_{\ell+1}^{(i-1)}(\nu)+m_{\ell+1}^{(i+1)}(\nu) .
\end{aligned}
$$

Recall that we have $m_{\ell+1}^{(i-1)}(\nu)>0$ by definition of $\ell^{(i-1)}(=\ell+1)$. Then the only possibility that is compatible with the above inequality is $m_{\ell+1}^{(i-1)}(\nu)=1$ and $m_{\ell+1}^{(i+1)}(\nu)=0$. Hence we have confirmed the relations (4) and (1).

Finally, since $m_{k}^{(i)}(\nu)=0$ and $m_{k}^{(i+1)}(\nu)=0$ for $\ell+1<k<\ell^{(i)}$, the relation (5.6) implies that $m_{k}^{(i-1)}(\nu)=0$ for $\ell+1<k<\ell^{(i)}$, since otherwise the relation would be strictly convex. Thus we have confirmed the relation $(2)$.

Proof of Proposition 5.11 for the case (5.4). The assertions $m_{k}^{(i+1)}(\nu)=0$ for $\ell<k<\ell^{(i)}$ are already proved in Corollary 5.15.

From (5.6) we have $P_{\ell^{(i)}-1}^{(i)}(\nu)=x_{\ell}+1$ since we have $\ell<\ell^{(i)}-1$ by the assumption $\left(\ell^{(i-1)}=\right)$ $\ell+1<\ell^{(i)}$. Since $\ell^{(i-1)} \leq \ell^{(i)}-1$ we have $P_{\ell^{(i)}-1}^{(i)}(\bar{\nu})=P_{\ell^{(i)}-1}^{(i)}(\nu)-1=x_{\ell}$. This completes the proof of Proposition 5.11 for the case (5.4). 
Thus we have confirmed $\overline{\widetilde{\nu}}=\widetilde{\bar{\nu}}$ in this case. In order to prove $\overline{\widetilde{J}}=\widetilde{\bar{J}}$, we need additional relations obtained in Corollary 5.15.

Proposition 5.16. Suppose that we have $\ell^{(i-1)}=\ell+1<\ell^{(i)}<\infty, m_{\ell+1}^{(i)}(\nu)=0$, the string $\left(\ell, x_{\ell}\right)$ is singular and $\widetilde{f}_{i}$ creates a singular string. Then we have $\overline{\widetilde{J}}=\widetilde{\bar{J}}$.

Proof. From Corollary 5.15 and Proposition 5.12 we see that there are no strings of length from $\ell+1$ to $\ell^{(i)}-1$ that are not touched by $\delta$ nor $\widetilde{f}_{i}$ during both $\delta \circ \widetilde{f}_{i}$ and $\widetilde{f}_{i} \circ \delta$. Thus it is enough to analyze the strings $\left(\ell, x_{\ell}\right)$ and $\left(\ell^{(i)}, x_{\ell^{(i)}}\right)$ of $(\nu, J)^{(i)}$.

Let us analyze the behavior of the string $\left(\ell, x_{\ell}\right)$. From the proof of Proposition 5.12 we have

$$
\begin{aligned}
& \left(\ell, x_{\ell}\right) \stackrel{\widetilde{f}_{i}}{\longmapsto}\left(\ell+1, x_{\ell}-1\right) \stackrel{\delta}{\stackrel{\delta}{\longmapsto}}\left(\ell, P_{\ell}^{(i)}(\overline{\widetilde{\nu}})\right), \\
& \left(\ell, x_{\ell}\right) \stackrel{\widetilde{f}_{i}}{\longmapsto}\left(\ell, x_{\ell}\right) \quad\left(\ell, x_{\ell}\right) .
\end{aligned}
$$

By $\ell<\ell^{(i-1)}$ we have $P_{\ell}^{(i)}(\overline{\widetilde{\nu}})=P_{\ell}^{(i)}(\nu)$. Recall that we have $P_{\ell}^{(i)}(\nu)=x_{\ell}$ by $(5.6)$. Hence we obtain $P_{\ell}^{(i)}(\overline{\widetilde{\nu}})=x_{\ell}$.

Next let us analyze the behavior of the string $\left(\ell^{(i)}, x_{\ell^{(i)}}\right)$. From the proof of Proposition 5.12 we have

$$
\begin{array}{llll}
\left(\ell^{(i)}, x_{\ell^{(i)}}\right) & \stackrel{\widetilde{f}_{i}}{\longmapsto}\left(\ell^{(i)}, x_{\ell^{(i)}}-2\right) & \stackrel{\delta}{\longmapsto} & \left(\ell^{(i)}, x_{\ell^{(i)}}-2\right), \\
\left(\ell^{(i)}, x_{\ell^{(i)}}\right) & \stackrel{\delta}{\longmapsto}\left(\ell^{(i)}-1, P_{\ell^{(i)}-1}^{(i)}(\bar{\nu})\right) & \stackrel{\widetilde{f}_{i}}{\longmapsto}\left(\ell^{(i)}, P_{\ell^{(i)}-1}^{(i)}(\bar{\nu})-1\right) .
\end{array}
$$

Recall that we have $\ell+1<\ell^{(i)}$. Thus the first $\widetilde{f}_{i}$ of $\delta \circ \widetilde{f}_{i}$ decreases the rigging of the string $\left(\ell^{(i)}, x_{\ell^{(i)}}\right)$ by 2 .

Let us check the coincidence of the final rigging of this case. Recall that we have $x_{\ell^{(i)}}=$ $P_{\ell^{(i)}}^{(i)}(\nu)$ since the string $\left(\ell^{(i)}, x_{\ell^{(i)}}\right)$ is singular by definition of $\ell^{(i)}$. We also have $P_{\ell^{(i)}}^{(i)}(\nu)=x_{\ell}+1$ by (5.6). Therefore we have $x_{\ell^{(i)}}-2=x_{\ell}-1$.

On the other hand, since $\ell^{(i-1)} \leq \ell^{(i)}-1$ we have $P_{\ell^{(i)}-1}^{(i)}(\bar{\nu})=P_{\ell^{(i)}-1}^{(i)}(\nu)-1$. From (5.6) we have $P_{\ell^{(i)}-1}^{(i)}(\nu)=x_{\ell}+1$. Therefore we have $P_{\ell^{(i)}-1}^{(i)}(\bar{\nu})-1=x_{\ell}-1$. Hence we conclude that $x_{\ell^{(i)}}-2=P_{\ell^{(i)}-1}^{(i)}(\bar{\nu})-1$.

Example 5.17. Consider the following rigged configuration $(\nu, J)$ of type $\left(B^{1,1}\right)^{\otimes 3} \otimes B^{1,3} \otimes$ $B^{2,1} \otimes B^{2,2} \otimes B^{3,1}$ of $D_{5}^{(1)}$
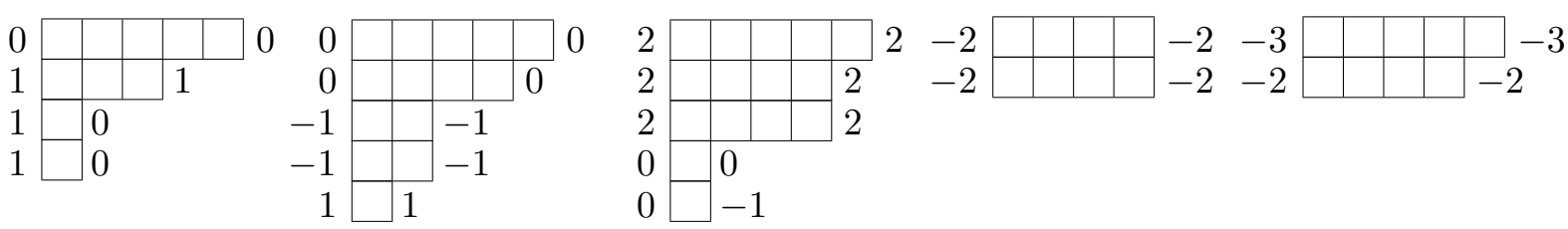

The corresponding tensor product $\Phi(\nu, J)$ is

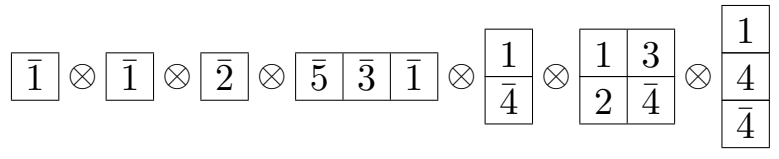

We see that $\widetilde{f}_{2}$ acts on the string $\left(\ell, x_{\ell}\right)=(2,-1)$ of $(\nu, J)^{(2)}$. Then $(\bar{\nu}, \bar{J})$ is 


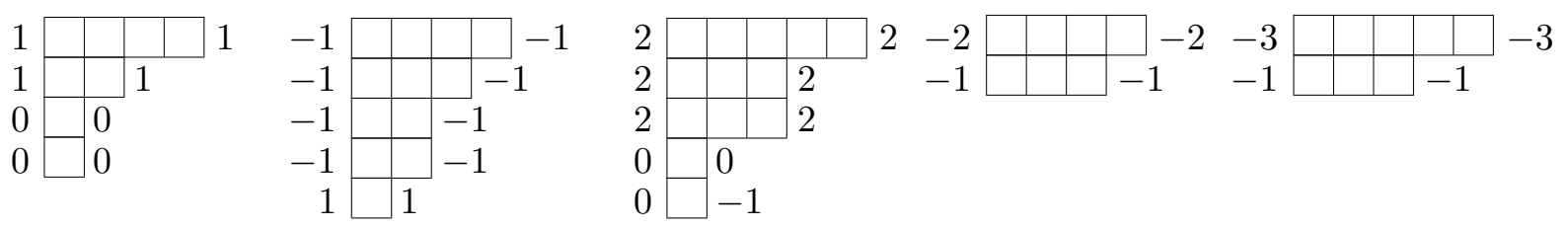

and $(\widetilde{\nu}, \widetilde{J})$ is

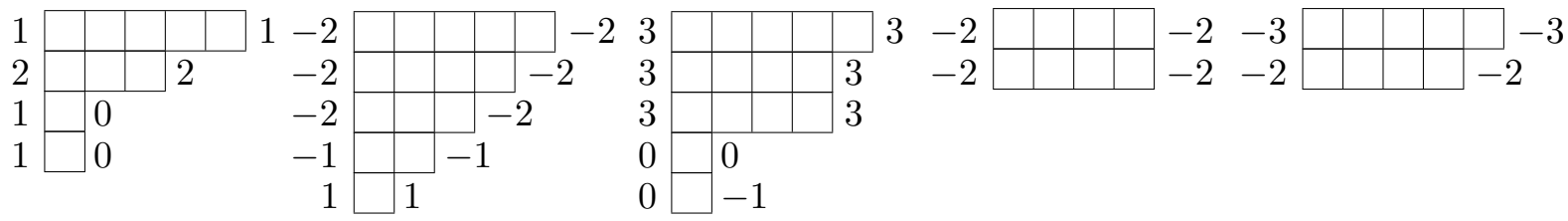

Note that we have $\ell^{(1)}=3=\ell+1<\ell^{(2)}=4$ and $m_{3}^{(2)}(\nu)=0$. Moreover, we see that the string $\left(\ell, x_{\ell}\right)$ is singular since we have $P_{2}^{(2)}=-1$, and $\widetilde{f}_{2}$ makes it into the singular string $(3,-2)$ of $(\widetilde{\nu}, \widetilde{J})^{(2)}$ since we have $P_{3}^{(2)}(\widetilde{\nu})=-2$. Hence this is the example for the present case. Then we have $P_{2}^{(2)}(\nu)=-1$ and $P_{3}^{(2)}(\nu)=P_{4}^{(2)}(\nu)=0$ where $\ell^{(2)}=4$ (see Proposition 5.14). We have $m_{3}^{(1)}(\nu)=1$ and $m_{3}^{(2)}(\nu)=m_{3}^{(3)}(\nu)=0$ (see Corollary 5.15). Finally $(\overline{\widetilde{\nu}}, \widetilde{\widetilde{J}})=(\widetilde{\bar{\nu}}, \widetilde{\bar{J}})$ is

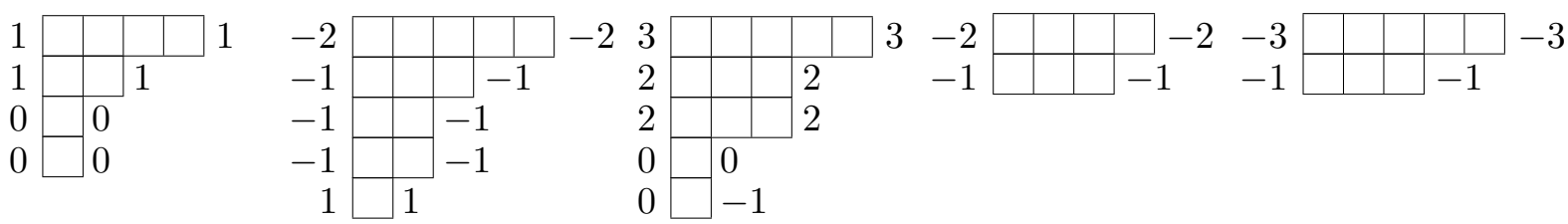

\subsubsection{Proof for the case (5.5)}

Under the present assumptions, let us show the following property.

Proposition 5.18. Suppose that we have $\ell^{(i-1)} \leq \ell+1<\ell^{(i)}<\infty, m_{\ell+1}^{(i)}(\nu)=0$, the string $\left(\ell, x_{\ell}\right)$ is non-singular and $\tilde{f}_{i}$ creates a singular string. Then we have

$$
P_{\ell}^{(i)}(\nu)=P_{\ell+1}^{(i)}(\nu)=\cdots=P_{\ell^{(i)}}^{(i)}(\nu)=x_{\ell}+1 .
$$

Proof. Let $j$ be the length of the shortest string of $\nu^{(i)}$ that satisfies $\ell<j$. By the assumption $m_{\ell+1}^{(i)}(\nu)=0$ we have $\ell+1<j \leq \ell^{(i)}$. From the convexity of $P_{k}^{(i)}(\nu)$ between $\ell \leq k \leq j$ we have

$$
P_{\ell+1}^{(i)}(\nu) \geq \min \left\{P_{\ell}^{(i)}(\nu), P_{j}^{(i)}(\nu)\right\} .
$$

In order to evaluate the minimum in (5.10), let us suppose if possible that $P_{\ell}^{(i)}(\nu)>P_{j}^{(i)}(\nu)$. Then the inequality $(5.10)$ reads $P_{\ell+1}^{(i)}(\nu) \geq P_{j}^{(i)}(\nu)$. Recall from Lemma 5.2(2) that we have $P_{\ell+1}^{(i)}(\nu) \leq x_{j}$ and by definition of the rigged configuration we have $x_{j} \leq P_{j}^{(i)}(\nu)$, that is, $P_{\ell+1}^{(i)}(\nu) \leq P_{j}^{(i)}(\nu)$. Combining the two inequalities we obtain $P_{\ell+1}^{(i)}(\nu)=P_{j}^{(i)}(\nu)$. By the assumption, we conclude that $P_{\ell}^{(i)}(\nu)>P_{\ell+1}^{(i)}(\nu)=P_{j}^{(i)}(\nu)$ which is in contradiction to the convexity relation of $P_{k}^{(i)}(\nu)$ between $\ell \leq k \leq j$.

Thus we assume that $P_{\ell}^{(i)}(\nu) \leq P_{j}^{(i)}(\nu)$. Then (5.10) gives $P_{\ell}^{(i)}(\nu) \leq P_{\ell+1}^{(i)}(\nu)$. We also have

$$
P_{\ell+1}^{(i)}(\nu) \stackrel{\text { by Lemma } 5.2(1)}{=} x_{\ell}+1 \stackrel{\text { since the string }\left(\ell, x_{\ell}\right) \text { is non-singular }}{\leq} P_{\ell}^{(i)}(\nu) .
$$


Thus we have $P_{\ell}^{(i)}(\nu)=P_{\ell+1}^{(i)}(\nu)$. Combining this with the inequality $P_{\ell}^{(i)}(\nu) \leq P_{j}^{(i)}(\nu)$ and using the convexity of $P_{k}^{(i)}(\nu)$ between $\ell \leq k \leq j$, we obtain

$$
P_{\ell}^{(i)}(\nu)=P_{\ell+1}^{(i)}(\nu)=\cdots=P_{j}^{(i)}(\nu)=x_{\ell}+1 .
$$

Suppose if possible that $j<\ell^{(i)}$. Since $\ell<j$ we have $P_{\ell+1}^{(i)}(\nu) \leq x_{j}$ by Lemma 5.2(2). Then we have $P_{\ell+1}^{(i)}(\nu) \leq x_{j} \leq P_{j}^{(i)}(\nu)$. By (5.11) we obtain $x_{j}=P_{j}^{(i)}(\nu)$, that is, the string $\left(j, x_{j}\right)$ is singular. Since $\ell^{(i-1)} \leq j<\ell^{(i)}$ this contradicts the definition of $\ell^{(i)}$. Hence we conclude that $j=\ell^{(i)}$.

Proof of Proposition 5.11 for the case (5.5). Since $\ell^{(i-1)}<\ell^{(i)}$ we have

$$
P_{\ell^{(i)}-1}^{(i)}(\bar{\nu})=P_{\ell^{(i)}-1}^{(i)}(\nu)-1=x_{\ell}
$$

where we have used (5.9). From the result at the end of the proof of the previous lemma, we see that $m_{k}^{(i)}(\nu)=0$ for $\ell<k<\ell^{(i)}$. Then the relation (5.9) implies that $m_{k}^{(i+1)}(\nu)=0$ for $\ell<k<\ell^{(i)}$ since the existence of such a string would imply that the relation (5.9) have to be strictly convex. Hence we complete the proof of Proposition 5.11.

By using the relation $\overline{\widetilde{\nu}}=\widetilde{\bar{\nu}}$ we show $\overline{\widetilde{J}}=\widetilde{\bar{J}}$. For this purpose we note the following facts which are the consequences of the proof of Proposition 5.18.

Corollary 5.19. Under the same assumptions with the previous proposition, we have the following relations.

(1) $m_{k}^{(a)}(\nu)=0$ for all $\ell<k<\ell^{(i)}$ and $a=i-1, i, i+1$,

(2) $\ell^{(i-1)} \leq \ell$.

Proof. (1) From $m_{k}^{(i)}(\nu)=m_{k}^{(i+1)}(\nu)=0$ for $\ell<k<\ell^{(i)}$, we see that if $m_{k}^{(i-1)}(\nu)>0$ for some $\ell<k<\ell^{(i)}$, then $P_{k}^{(i)}(\nu)$ becomes strictly convex around such the $k$, which is in contradiction to the relation (5.9). Thus we obtain the assertion.

(2) If $\ell<\ell^{(i-1)}$, we have $m_{\ell^{(i-1)}}^{(i-1)}(\nu)>0$ which is in contradiction to the previous assertion since $\ell^{(i-1)}<\ell^{(i)}$. Thus we have $\ell^{(i-1)} \leq \ell$.

Proposition 5.20. Suppose that we have $\ell^{(i-1)} \leq \ell+1<\ell^{(i)}<\infty, m_{\ell+1}^{(i)}(\nu)=0$, the string $\left(\ell, x_{\ell}\right)$ is non-singular and $\widetilde{f}_{i}$ creates a singular string. Then we have $\overline{\widetilde{J}}=\widetilde{\bar{J}}$.

Proof. By Proposition 5.12 and Corollary 5.19(1) we see that it is enough to check the coincidence of riggings for the strings $\left(\ell, x_{\ell}\right)$ and $\left(\ell^{(i)}, x_{\ell^{(i)}}\right)$ that are touched by $\delta$ and $\widetilde{f}_{i}$.

Let us analyze the change of the string $\left(\ell, x_{\ell}\right)$. Since $\ell+1<\ell^{(i)}$ we have

$$
\begin{aligned}
& \left(\ell, x_{\ell}\right) \stackrel{\widetilde{f}_{i}}{\longmapsto}\left(\ell+1, x_{\ell}-1\right) \stackrel{\delta}{\longmapsto}\left(\ell, P_{\ell}^{(i)}(\overline{\widetilde{\nu}})\right), \\
& \left(\ell, x_{\ell}\right) \stackrel{\delta}{\longmapsto}\left(\ell, x_{\ell}\right) \stackrel{\widetilde{f}_{i}}{\longmapsto}\left(\ell, x_{\ell}\right) .
\end{aligned}
$$

Since $\ell^{(i-1)} \leq \ell$ by Corollary $5.19(2)$ we have $P_{\ell}^{(i)}(\overline{\widetilde{\nu}})=P_{\ell}^{(i)}(\nu)-1$. From (5.9), we have $P_{\ell}^{(i)}(\nu)=x_{\ell}+1$. Hence we obtain $P_{\ell}^{(i)}(\overline{\widetilde{\nu}})=x_{\ell}$.

The analysis of the string $\left(\ell^{(i)}, x_{\ell^{(i)}}\right)$ is the same with the corresponding part of the proof of Proposition 5.16 if we replace (5.6) there by (5.9). 


\subsection{Proof for Case C (3)}

As the final step of the proof for Case $\mathrm{C}$, let us treat the case where $\ell+1<\ell^{(i)}$ and $\widetilde{f}_{i}$ creates a non-singular string.

Proposition 5.21. Assume that $\ell^{(i-1)} \leq \ell+1<\ell^{(i)}<\infty$. If $\tilde{f}_{i}$ acting on $(\nu, J)$ creates a non-singular string, then the following relation is satisfied:

$$
P_{\ell^{(i)}-1}^{(i)}(\bar{\nu})>x_{\ell}
$$

Proof. In this case we have

$$
P_{\ell^{(i)}-1}^{(i)}(\bar{\nu})=P_{\ell^{(i)}-1}^{(i)}(\nu)-1
$$

since $\ell^{(i-1)} \leq \ell^{(i)}-1$.

Throughout the proof of this proposition, let $j$ be the largest integer such that $j<\ell^{(i)}$ and $m_{j}^{(i)}(\nu)>0$. Since $m_{\ell}^{(i)}(\nu)>0$, we have $\ell \leq j \leq \ell^{(i)}-1$. We divide the proof into four cases.

Case $j=\ell^{(i)}-1$. Let us denote the corresponding string as $\left(\ell^{(i)}-1, x_{\ell^{(i)}-1}\right)$. Since $\ell^{(i-1)}<$ $\ell^{(i)}$ we have $\ell^{(i-1)} \leq \ell^{(i)}-1$ which implies that the string $\left(\ell^{(i)}-1, x_{\ell^{(i)}-1}\right)$ is non-singular by definition of $\ell^{(i)}$. Thus we have $x_{\ell^{(i)}-1}<P_{\ell^{(i)}-1}^{(i)}(\nu)$. On the other hand, from the assumption $\ell+1<\ell^{(i)}$, we have $x_{\ell}<x_{\ell^{(i)}-1}$ since $\tilde{f}_{i}$ chooses the string $\left(\ell, x_{\ell}\right)$ which is strictly shorter than the string $\left(\ell^{(i)}-1, x_{\ell^{(i)}-1}\right)$. Combining both inequalities we obtain $x_{\ell}<P_{\ell^{(i)}-1}^{(i)}(\nu)-1$. Hence we obtain $x_{\ell}<P_{\ell^{(i)}-1}^{(i)}(\bar{\nu})$.

Case $\ell<j<\ell^{(i)}-1$. In this case the string $\left(j, x_{j}\right)$ have to be non-singular since $j$ satisfies $\ell^{(i-1)} \leq \ell+1 \leq j<\ell^{(i)}$. Then we have

$$
P_{j}^{(i)}(\nu) \stackrel{\text { since the string }\left(j, x_{j}\right) \text { is non-singular }}{>} x_{j} \text { since } \widetilde{f}_{i} \text { acts on the string }\left(\ell, x_{\ell}\right) \text { although we have } j>\ell x_{\ell} .
$$

In particular we have $P_{j}^{(i)}(\nu)>x_{\ell}+1$. Similarly let us consider the string $\left(\ell^{(i)}, P_{\ell^{(i)}}^{(i)}(\nu)\right)$ whose existence is assured by the definition of $\ell^{(i)}$. Then its rigging must satisfy $P_{\ell^{(i)}}^{(i)}(\nu)>x_{\ell}$ since its length satisfies $\ell^{(i)}>\ell$. From the convexity relation of $P_{k}^{(i)}(\nu)$ between $j \leq k \leq \ell^{(i)}$ we have

$$
P_{\ell^{(i)}-1}^{(i)}(\nu) \geq \min \left\{P_{j}^{(i)}(\nu), P_{\ell^{(i)}}^{(i)}(\nu)\right\}>x_{\ell} .
$$

Suppose if possible that $P_{\ell^{(i)}-1}^{(i)}(\nu)=x_{\ell}+1$. Then we would have $P_{j}^{(i)}(\nu)>P_{\ell^{(i)}-1}^{(i)}(\nu) \leq P_{\ell^{(i)}}^{(i)}(\nu)$ which is forbidden by the convexity of the vacancy numbers. Thus we have $P_{\ell^{(i)}-1}^{(i)}(\nu)>x_{\ell}+1$. By (5.13) we have $P_{\ell^{(i)}-1}^{(i)}(\bar{\nu})>x_{\ell}$.

Case $\boldsymbol{j}=\ell$ and $\left(\ell, x_{\ell}\right)$ is non-singular. In this case we have $P_{\ell}^{(i)}(\nu)>x_{\ell}$ since the string $\left(\ell, x_{\ell}\right)$ is non-singular. Let us consider the string $\left(\ell^{(i)}, P_{\ell^{(i)}}^{(i)}(\nu)\right)$. Then its rigging must satisfy $P_{\ell^{(i)}}^{(i)}(\nu)>x_{\ell}$ since its length satisfy $\ell^{(i)}>\ell$. Now we invoke the convexity of $P_{k}^{(i)}(\nu)$ between $\ell=j \leq k \leq \ell^{(i)}$ to conclude that $P_{\ell^{(i)}-1}^{(i)}(\nu)>x_{\ell}$.

Suppose if possible that $P_{\ell^{(i)}-1}^{(i)}(\nu)=x_{\ell}+1$. Again by the convexity relation the only possibility is $P_{\ell}^{(i)}(\nu)=\cdots=P_{\ell^{(i)}}^{(i)}=x_{\ell}+1$. In particular, we have $P_{\ell+1}^{(i)}(\nu)=x_{\ell}+1$ and by Lemma $5.2(1)$ this implies that $\tilde{f}_{i}$ makes a singular string. This is in contradiction to the assumption that $\tilde{f}_{i}$ makes a non-singular string. Thus we have $P_{\ell^{(i)}-1}^{(i)}(\nu)>x_{\ell}+1$. By (5.13) we have $P_{\ell^{(i)}-1}^{(i)}(\bar{\nu})>x_{\ell}$. 
Case $\boldsymbol{j}=\ell$ and $\left(\ell, \boldsymbol{x}_{\ell}\right)$ is singular. In this case we have $P_{\ell}^{(i)}(\nu)=x_{\ell}$ since the string $\left(\ell, x_{\ell}\right)$ is singular. Also the rigging for the string $\left(\ell^{(i)}, P_{\ell^{(i)}}^{(i)}(\nu)\right)$ must satisfy $P_{\ell^{(i)}}^{(i)}(\nu)>x_{\ell}$ since $\ell^{(i)}>\ell$. Then from the convexity relation of $P_{k}^{(i)}(\nu)$ between $\ell \leq k \leq \ell^{(i)}$ we have

$$
P_{\ell^{(i)}-1}^{(i)}(\nu) \geq \min \left\{P_{\ell}^{(i)}(\nu), P_{\ell^{(i)}}^{(i)}(\nu)\right\}=x_{\ell} .
$$

If $P_{\ell^{(i)}-1}^{(i)}(\nu)=x_{\ell}$ we have $P_{\ell}^{(i)}(\nu)=P_{\ell^{(i)}-1}^{(i)}(\nu)<P_{\ell^{(i)}}^{(i)}(\nu)$ which is in contradiction to the convexity relation since we have $\ell<\ell^{(i)}-1$ by the assumption $\ell+1<\ell^{(i)}$. Suppose if possible that $P_{\ell^{(i)}-1}^{(i)}(\nu)=x_{\ell}+1$. Since $x_{\ell}<P_{\ell^{(i)}}^{(i)}(\nu)$ we have $P_{\ell^{(i)}-1}^{(i)}(\nu) \leq P_{\ell^{(i)}}^{(i)}(\nu)$. Then the only possibility that is compatible with the convexity relation is $x_{\ell}=P_{\ell}^{(i)}(\nu)<P_{\ell+1}^{(i)}(\nu)=\cdots=$ $P_{\ell^{(i)}}^{(i)}(\nu)=x_{\ell}+1$. In particular, we have $P_{\ell+1}^{(i)}(\nu)=x_{\ell}+1$. However, by Lemma 5.2(1), this implies that $\tilde{f}_{i}$ creates a singular string, which is the contradiction. Thus we conclude that $P_{\ell^{(i)}-1}^{(i)}(\nu)>x_{\ell}+1$. By $(5.13)$ we have $P_{\ell^{(i)}-1}^{(i)}(\bar{\nu})>x_{\ell}$, which completes the whole proof of Proposition 5.21.

Proposition 5.22. Assume that $\ell^{(i-1)} \leq \ell+1<\ell^{(i)}<\infty$ and $\tilde{f}_{i}$ acting on $(\nu, J)$ creates a non-singular string. Then we have the following identities:

$$
\text { (1) } \quad \overline{\widetilde{\nu}}=\widetilde{\bar{\nu}}, \quad(2) \quad \overline{\widetilde{J}}=\widetilde{\bar{J}} .
$$

Proof. (1) We show that $\widetilde{f}_{i}$ acts on the same string $\left(\ell, x_{\ell}\right)$ in both $(\nu, J)$ and $(\bar{\nu}, \bar{J})$. For $\delta$ creates the strings $\left(\ell^{(i)}-1, P_{\ell^{(i)}-1}^{(i)}(\bar{\nu})\right)$ and $\left(\ell_{(i)}-1, P_{\ell_{(i)}-1}^{(i)}(\bar{\nu})\right)$. Then by $(5.12)$ we have $P_{\ell^{(i)}-1}^{(i)}(\bar{\nu})>x_{\ell}$ in this case. Recall also that we have $P_{\ell_{(i)}-1}^{(i)}(\bar{\nu})>x_{\ell}$ if $\ell^{(i)}<\ell_{(i)}$ by Proposition 5.8. Therefore the string $\left(\ell, x_{\ell}\right)$ remains as the string with the smallest rigging of the largest length in $(\bar{\nu}, \bar{J})$. Thus $\widetilde{f}_{i}$ acts on the string $\left(\ell, x_{\ell}\right)$ of $(\bar{\nu}, \bar{J})$.

Also, since $\widetilde{f}_{i}$ creates the non-singular string and does not change other coriggings, $\delta$ chooses the same strings for both $(\nu, J)$ and $(\widetilde{\nu}, \widetilde{J})$. Thus we have $\overline{\widetilde{\nu}}=\widetilde{\bar{\nu}}$.

(2) Since $\widetilde{f}_{i}$ acts on the string $\left(\ell, x_{\ell}\right)$ in both cases, we have to analyze the strings $\left(\ell, x_{\ell}\right)$, $\left(\ell^{(i)}, P_{\ell^{(i)}}^{(i)}(\nu)\right)$ and $\left(\ell_{(i)}, P_{\ell_{(i)}}^{(i)}(\nu)\right)$. The string $\left(\ell, x_{\ell}\right)$ behaves as follows:

$$
\begin{array}{lll}
\left(\ell, x_{\ell}\right) \stackrel{\widetilde{f}_{i}}{\longmapsto}\left(\ell+1, x_{\ell}-1\right) & \stackrel{\delta}{\longmapsto}\left(\ell+1, x_{\ell}-1\right), \\
\left(\ell, x_{\ell}\right) \stackrel{\widetilde{f}_{i}}{\longmapsto} & \stackrel{\longmapsto}{\longmapsto}\left(\ell, x_{\ell}\right) & \left(\ell+1, x_{\ell}-1\right) .
\end{array}
$$

Hence the assertion follows. On the other hand, the string $\left(\ell^{(i)}, P_{\ell^{(i)}}^{(i)}(\nu)\right)$ behaves as follows:

$$
\begin{array}{llll}
\left(\ell^{(i)}, P_{\ell^{(i)}}^{(i)}(\nu)\right) & \stackrel{\widetilde{f}_{i}}{\longmapsto}\left(\ell^{(i)}, P_{\ell^{(i)}}^{(i)}(\widetilde{\nu})\right) & \stackrel{\delta}{\longmapsto} & \left(\ell^{(i)}-1, P_{\ell^{(i)}-1}^{(i)}(\overline{\widetilde{\nu}})\right), \\
\left(\ell^{(i)}, P_{\ell^{(i)}}^{(i)}(\nu)\right) & \stackrel{\delta}{\longmapsto}\left(\ell^{(i)}-1, P_{\ell^{(i)}-1}^{(i)}(\bar{\nu})\right) & \stackrel{\widetilde{f}_{i}}{\longmapsto} & \left(\ell^{(i)}-1, P_{\ell^{(i)}-1}^{(i)}(\widetilde{\bar{\nu}})\right) .
\end{array}
$$

By $\overline{\widetilde{\nu}}=\widetilde{\bar{\nu}}$ we obtain the desired fact. The analysis of $\left(\ell_{(i)}, P_{\ell_{(i)}}^{(i)}(\nu)\right)$ is similar. Hence we have $\overline{\widetilde{J}}=\widetilde{\bar{J}}$.

\subsection{Proof for Case D: preliminary steps}

\subsubsection{Classification}

Recall that the defining condition for Case $\mathrm{D}$ is $\ell^{(i)}=\ell$. For the proof, it is convenient to further divide the case into the following four cases: 
(1) $\ell=\ell^{(i)}<\ell_{(i)}$ and $m_{\ell}^{(i)}(\nu)>1$,

(2) $\ell=\ell^{(i)}=\ell_{(i)}$ and $m_{\ell}^{(i)}(\nu)>2$,

(3) $\ell=\ell^{(i)}<\ell_{(i)}$ and $m_{\ell}^{(i)}(\nu)=1$,

(4) $\ell=\ell^{(i)}=\ell_{(i)}$ and $m_{\ell}^{(i)}(\nu)=2$.

Note that $\ell^{(i)}=\ell_{(i)}$ automatically requires $m_{\ell^{(i)}}^{(i)}(\nu) \geq 2$.

\subsubsection{A common property for Case D}

For the proof of Case D, we will need the following result.

Proposition 5.23. Assume that $\ell^{(i)}=\ell$. Then the following relation holds:

$$
P_{\ell-1}^{(i)}(\bar{\nu}) \geq x_{\ell} .
$$

Proof. Throughout the proof of this proposition, let $j$ be the largest integer such that $j<\ell$ and $m_{j}^{(i)}(\nu)>0$. If there is no such $j$, set $j=0$. We divide the proof into the following four cases:

(a) $j=\ell-1$,

(b) $j<\ell-1, \ell^{(i-1)}=\ell$,

(c) $j<\ell-1, \ell^{(i-1)}<\ell$ and $\ell^{(i-1)} \leq j$,

(d) $j<\ell-1, \ell^{(i-1)}<\ell$ and $j<\ell^{(i-1)}$.

Case (a). Denote the corresponding string of $(\nu, J)^{(i)}$ as $\left(\ell-1, x_{\ell-1}\right)$. Then we have $x_{\ell} \leq x_{\ell-1}$ by definition of $\ell$. Suppose that $\ell^{(i-1)}<\ell$. Since we have $\ell^{(i-1)} \leq \ell-1<\ell^{(i)}$ the string $\left(\ell-1, x_{\ell-1}\right)$ cannot be singular. Thus we have $P_{\ell-1}^{(i)}(\nu)>x_{\ell}$ by $P_{\ell-1}^{(i)}(\nu)>x_{\ell-1} \geq x_{\ell}$. Thus we obtain $P_{\ell-1}^{(i)}(\bar{\nu}) \geq x_{\ell}$ since $P_{\ell-1}^{(i)}(\bar{\nu})=P_{\ell-1}^{(i)}(\nu)-1$ by $\ell^{(i-1)} \leq \ell-1$.

Next suppose that $\ell^{(i-1)}=\ell$. In this case the string $\left(\ell-1, x_{\ell-1}\right)$ can be singular. Therefore we have $P_{\ell-1}^{(i)}(\nu) \geq x_{\ell}$ by $P_{\ell-1}^{(i)}(\nu) \geq x_{\ell-1} \geq x_{\ell}$. From $\ell-1<\ell^{(i-1)}$ we have $P_{\ell-1}^{(i)}(\bar{\nu})=P_{\ell-1}^{(i)}(\nu)$. Thus we obtain $P_{\ell-1}^{(i)}(\bar{\nu}) \geq x_{\ell}$.

Case (b). By $j<\ell$ we have $P_{j}^{(i)}(\nu) \geq x_{j} \geq x_{\ell}$. Note that this relation is valid even if $j=0$ since we have $P_{0}^{(i)}(\nu)=0 \geq x_{\ell}$ by $\ell=\ell^{(i)}>0$. Recall that we also have $P_{\ell}^{(i)}(\nu) \geq x_{\ell}$. Thus by the convexity of $P_{k}^{(i)}(\nu)$ between $j \leq k \leq \ell$ we have $P_{\ell-1}^{(i)}(\nu) \geq x_{\ell}$. Since $\ell-1<\ell^{(i-1)}$ we conclude that $P_{\ell-1}^{(i)}(\bar{\nu}) \geq x_{\ell}$.

Case (c). We can write the defining condition of case (c) as $\ell^{(i-1)} \leq j<\ell-1$. Since $\ell^{(i-1)} \leq j<\ell^{(i)}$ the string $\left(j, x_{j}\right)$ is non-singular. Thus we have $P_{j}^{(i)}(\nu)>x_{\ell}$ by $P_{j}^{(i)}(\nu)>$ $x_{j} \geq x_{\ell}$. We also have $P_{\ell}^{(i)}(\nu) \geq x_{\ell}$. Suppose if possible that $P_{\ell-1}^{(i)}(\nu)=x_{\ell}$. Then we have $P_{j}^{(i)}(\nu)>P_{\ell-1}^{(i)}(\nu) \leq P_{\ell}^{(i)}(\nu)$ which is in contradiction to the convexity relation of $P_{k}^{(i)}(\nu)$ between $j \leq k \leq \ell$. Thus we have $P_{\ell-1}^{(i)}(\nu)>x_{\ell}$. By $\ell^{(i-1)}<\ell-1$ and $\ell=\ell^{(i)}$ we have $P_{\ell-1}^{(i)}(\bar{\nu}) \geq x_{\ell}$.

Case (d). We can write the defining condition of case $(\mathrm{d})$ as $j<\ell^{(i-1)}<\ell$. In this case the string $\left(j, x_{j}\right)$ can be singular. Thus we have $P_{j}^{(i)}(\nu) \geq x_{\ell}$ by $P_{j}^{(i)}(\nu) \geq x_{j} \geq x_{\ell}$. We also have $P_{\ell}^{(i)}(\nu) \geq x_{\ell}$. Suppose if possible that $P_{\ell-1}^{(i)}(\nu)=x_{\ell}$. Then by the convexity relation of $P_{k}^{(i)}(\nu)$ between $j \leq k \leq \ell$, the only possibility is $P_{j}^{(i)}(\nu)=\cdots=P_{\ell}^{(i)}(\nu)=x_{\ell}$. However this is in contradiction to the fact that $P_{k}^{(i)}(\nu)$ is a strictly convex function between $\ell^{(i-1)}-1 \leq k \leq \ell^{(i-1)}+1$ due to the existence of the length $\ell^{(i-1)}$ row at $\nu^{(i-1)}$. Thus we have $P_{\ell-1}^{(i)}(\nu)>x_{\ell}$. By $\ell^{(i-1)} \leq \ell-1$ we have $P_{\ell-1}^{(i)}(\bar{\nu}) \geq x_{\ell}$. 


\subsection{Proof for Case D (1)}

In this section, let us consider the first case $\ell=\ell^{(i)}<\ell_{(i)}$ and $m_{\ell}^{(i)}(\nu)>1$. Throughout this subsection, let $j$ be the largest integer satisfying $j<\ell_{(i)}$ and $m_{j}^{(i)}(\nu)>0$. From $\ell=\ell^{(i)}<\ell_{(i)}$, we see that $\ell=\ell^{(i)} \leq j$. The situation depends on whether $\ell<j$ or $\ell=j$.

\subsubsection{The case $\ell<j$}

Proposition 5.24. Assume that $\ell=\ell^{(i)}<\ell_{(i)}, m_{\ell}^{(i)}(\nu)>1$ and $\ell<j$. Then we have the following identities:
(1) $\quad \overline{\widetilde{\nu}}=\widetilde{\bar{\nu}}$,
(2) $\overline{\widetilde{J}}=\widetilde{\bar{J}}$.

Proof. (1) Let us analyze the action of $\widetilde{f}_{i}$ before and after the application of $\delta$. In this case we can choose two distinct length $\ell$ strings $\left(\ell, x_{\ell}\right)$ and $\left(\ell, P_{\ell}^{(i)}(\nu)\right)$ of $(\nu, J)^{(i)}$ where $\widetilde{f}_{i}$ will act on the former one and $\delta$ will act on the latter one. Here note that if $x_{\ell}=P_{\ell}^{(i)}(\nu)$ then all the riggings for the length $\ell$ strings are the same since the minimal value of the corresponding riggings is $x_{\ell}$ and the maximal one is $P_{\ell}^{(i)}(\nu)$.

By Proposition 5.23 we have $P_{\ell^{(i)}-1}^{(i)}(\bar{\nu}) \geq x_{\ell}$. Thus the string $\left(\ell^{(i)}-1, P_{\ell^{(i)}-1}^{(i)}(\bar{\nu})\right)$ is shorter than $\left(\ell, x_{\ell}\right)$ of $(\bar{\nu}, \bar{J})^{(i)}$ and its rigging is larger than or equal to $x_{\ell}$. Also, by $\ell<j$, we can use the same arguments of Proposition 5.8 to show $P_{\ell_{(i)}-1}^{(i)}(\bar{\nu})>x_{\ell}$. Thus the string $\left(\ell_{(i)}-1, P_{\ell_{(i)}-1}^{(i)}(\bar{\nu})\right)$ has the rigging that is strictly larger than $x_{\ell}$. Therefore $\tilde{f}_{i}$ will act on the same string $\left(\ell, x_{\ell}\right)$ in both $(\nu, J)$ and $(\bar{\nu}, \bar{J})$.

Let us analyze $\delta$. Since $\tilde{f}_{i}$ will not change the corigging of the string $\left(\ell^{(i)}, P_{\ell^{(i)}}^{(i)}(\nu)\right)$ of $(\nu, J)^{(i)}$, it remains as the shortest possible string in $(\widetilde{\nu}, \widetilde{J})^{(i)}$ starting from $\widetilde{\ell}^{(i-1)}=\ell^{(i-1)}$. So $\delta$ chooses this string after the application of $\tilde{f}_{i}$ too. Let us show that the string $\left(\ell_{(i)}, P_{\ell_{(i)}}^{(i)}(\nu)\right)$ is chosen by $\delta$ in both before and after the application of $\widetilde{f}_{i}$. Recall that we have two length $\ell$ strings $\left(\ell, x_{\ell}\right)$ and $\left(\ell, P_{\ell}^{(i)}(\nu)\right)$ of $(\nu, J)^{(i)}$ where $\tilde{f}_{i}$ acts on the former one and $\delta$ acts on the latter one.

(i) Consider the case $P_{\ell}^{(i)}(\nu)=x_{\ell}$, that is, the string $\left(\ell, x_{\ell}\right)$ is singular. Then the assumptions $\ell^{(i)}<\ell_{(i)}$ and $m_{\ell^{(i)}}^{(i)}(\nu) \geq 2$ implies that $\ell^{(i)}<\ell_{(i+1)}$, as otherwise we would have $\ell^{(i)}=\ell_{(i)}$. Then a subtlety could occur only if $\ell_{(i)}>\ell_{(i+1)}=\ell+1$ and $\tilde{f}_{i}$ creates a singular string of length $\ell+1$. So suppose if possible that such a situation happens. In this case, we have $P_{\ell+1}^{(i)}(\nu)=x_{\ell}+1$ by Lemma 5.2.

Suppose if possible that $m_{\ell+1}^{(i)}(\nu)>0$. Then the riggings for such string have to be $x_{\ell}+1$ by the minimality of $x_{\ell}$. Thus the length $\ell+1$ strings are singular. However this is in contradiction to the present assumption $\ell_{(i)}>\ell+1$. Thus we have $m_{\ell+1}^{(i)}(\nu)=0$.

Next we show $m_{\ell+1}^{(i+1)}(\nu)=1$. Let $j^{\prime}$ be the smallest integer such that $\ell+1 \leq j^{\prime}$ and $m_{j^{\prime}}^{(i)}(\nu)>0$. Since $m_{\ell+1}^{(i)}(\nu)=0$ and $\ell_{(i)}>\ell+1$ we have $\ell+1<j^{\prime} \leq \ell_{(i)}$. Also we have $j^{\prime} \leq j$ by the assumption $\ell<j$. Recall that we have $P_{\ell}^{(i)}(\nu)=x_{\ell}$ and $P_{\ell+1}^{(i)}(\nu)=x_{\ell}+1$. By the minimality of $x_{\ell}$ we have $P_{\ell+2}^{(i)}(\nu) \geq x_{\ell}+1$ as otherwise we would have $P_{j^{\prime}}^{(i)}(\nu) \leq x_{\ell}$. Let us write $P_{\ell+2}^{(i)}(\nu)=x_{\ell}+1+\varepsilon$ with $\varepsilon \geq 0$. Then the inequality of Lemma 3.13 with $l=\ell+1$ reads

$$
\begin{aligned}
& -P_{\ell}^{(i)}(\nu)+2 P_{\ell+1}^{(i)}(\nu)-P_{\ell+2}^{(i)}(\nu)=1-\varepsilon \\
& \quad \geq m_{\ell+1}^{(i-1)}(\nu)-2 m_{\ell+1}^{(i)}(\nu)+m_{\ell+1}^{(i+1)}(\nu)=m_{\ell+1}^{(i-1)}(\nu)+m_{\ell+1}^{(i+1)}(\nu) .
\end{aligned}
$$


Since we have $m_{\ell+1}^{(i+1)}(\nu)>0$ by $\ell_{(i+1)}=\ell+1$, the only possibility is $\varepsilon=0, m_{\ell+1}^{(i-1)}(\nu)=0$ and $m_{\ell+1}^{(i+1)}(\nu)=1$.

Since $\ell<j^{\prime}$ we have $P_{j^{\prime}}^{(i)}(\nu) \geq x_{j^{\prime}}>x_{\ell}$. Then from the convexity relation of $P_{k}^{(i)}(\nu)$ between $\ell \leq k \leq j^{\prime}$, the only possibility that is compatible with $P_{\ell+1}^{(i)}(\nu)=P_{\ell+2}^{(i)}(\nu)=x_{\ell}+1$ is $P_{\ell+1}^{(i)}(\nu)=\cdots=P_{j^{\prime}}^{(i)}(\nu)=x_{\ell}+1$. Since $x_{j^{\prime}}>x_{\ell}$, we see that $x_{j^{\prime}}=P_{j^{\prime}}^{(i)}(\nu)$, in particular, the corresponding string is singular. Since $j^{\prime} \geq \ell+1=\ell_{(i+1)}$, we conclude that $j^{\prime}=\ell_{(i)}$. However this relation contradicts the requirement $j^{\prime} \leq j<\ell_{(i)}$.

(ii) Consider the case $P_{\ell}^{(i)}(\nu)>x_{\ell}$. Recall that we have $\ell^{(i)} \leq \ell_{(i+1)}$. Again a subtlety could occur only if $\ell_{(i)}>\ell_{(i+1)}=\ell+1$ and $\widetilde{f}_{i}$ creates a singular string of length $\ell+1$. So suppose if possible that such a situation happens. By Lemma 5.2, we have $P_{\ell+1}^{(i)}(\nu)=x_{\ell}+1$. As in the previous case, we have $m_{\ell+1}^{(i)}(\nu)=0$. Let $j^{\prime}$ be the smallest integer such that $\ell+1 \leq j^{\prime}$ and $m_{j^{\prime}}^{(i)}(\nu)>0$. Then, as in the previous case, we have $\ell+1<j^{\prime} \leq \ell_{(i)}$ and $j^{\prime} \leq j$. Then from the convexity relation of $P_{k}^{(i)}(\nu)$ between $\ell \leq k \leq j^{\prime}$ the only possibility that is compatible with the relations $P_{\ell}^{(i)}(\nu)>x_{\ell}, P_{\ell+1}^{(i)}(\nu)=x_{\ell}+1$ and $P_{j^{\prime}}^{(i)}(\nu)>x_{\ell}$ is $P_{\ell}^{(i)}(\nu)=\cdots=P_{j^{\prime}}^{(i)}(\nu)=x_{\ell}+1$. On the other hand, by $\ell<j^{\prime}$ we have $P_{j^{\prime}}^{(i)}(\nu) \geq x_{j^{\prime}}>x_{\ell}$. Therefore we have $P_{j^{\prime}}^{(i)}(\nu)=x_{j^{\prime}}=x_{\ell}+1$ and, in particular, the corresponding string is singular. Since $\ell+1=\ell_{(i+1)} \leq j^{\prime}$ we conclude that $j^{\prime}=\ell_{(i)}$. This is a contradiction since we have $j^{\prime} \leq j<\ell_{(i)}$.

Therefore $\delta$ will act on the same strings of lengths $\ell^{(i)}$ and $\ell_{(i)}$ in both $(\nu, J)^{(i)}$ and $(\widetilde{\nu}, \widetilde{J})^{(i)}$. Thus we conclude that $\overline{\widetilde{\nu}}=\widetilde{\bar{\nu}}$ in this case.

(2) It is enough to consider the three strings $\left(\ell, x_{\ell}\right),\left(\ell^{(i)}, P_{\ell^{(i)}}^{(i)}(\nu)\right)$ and $\left(\ell_{(i)}, P_{\ell_{(i)}}^{(i)}(\nu)\right)$. As for $\left(\ell, x_{\ell}\right)$, we have

$$
\begin{aligned}
& \left(\ell, x_{\ell}\right) \stackrel{\widetilde{f}_{i}}{\longmapsto}\left(\ell+1, x_{\ell}-1\right) \stackrel{\delta}{\longmapsto}\left(\ell+1, x_{\ell}-1\right), \\
& \left(\ell, x_{\ell}\right) \stackrel{\delta}{\longmapsto}\left(\ell, x_{\ell}\right) \quad \stackrel{\widetilde{f}_{i}}{\longmapsto}\left(\ell+1, x_{\ell}-1\right),
\end{aligned}
$$

and as for $\left(\ell^{(i)}, P_{\ell^{(i)}}^{(i)}(\nu)\right)$ we have

$$
\begin{array}{lllll}
\left(\ell^{(i)}, P_{\ell^{(i)}}^{(i)}(\nu)\right) & \stackrel{\tilde{f}_{i}}{\longmapsto}\left(\ell^{(i)}, P_{\ell^{(i)}}^{(i)}(\widetilde{\nu})\right) & \stackrel{\delta}{\longmapsto} & \left(\ell^{(i)}-1, P_{\ell^{(i)}-1}^{(i)}(\overline{\widetilde{\nu}})\right), \\
\left(\ell^{(i)}, P_{\ell^{(i)}}^{(i)}(\nu)\right) & \stackrel{\delta}{\longmapsto}\left(\ell^{(i)}-1, P_{\ell^{(i)}-1}^{(i)}(\bar{\nu})\right) & \stackrel{\widetilde{f}_{i}}{\longmapsto} & \left(\ell^{(i)}-1, P_{\ell^{(i)}-1}^{(i)}(\widetilde{\bar{\nu}})\right) .
\end{array}
$$

The situation for $\left(\ell_{(i)}, P_{\ell_{(i)}}^{(i)}(\nu)\right)$ is similar. Thus we have $\overline{\widetilde{J}}=\widetilde{\bar{J}}$.

\subsubsection{The case $\ell=j$}

We follow the classification of Lemma 5.1.

Proposition 5.25. Assume that $\ell=\ell^{(i)}<\ell^{(i+1)}=\cdots=\ell_{(i)}, m_{\ell}^{(i)}(\nu)>1$ and $\ell=j$. Then we have the following identities:

$$
\text { (1) } \quad \overline{\widetilde{\nu}}=\widetilde{\bar{\nu}}, \quad(2) \quad \overline{\widetilde{J}}=\widetilde{\bar{J}} .
$$

Proof. (1) In this case, we can use the same arguments of the proof of Case (V) of Proposition 5.8 to show $P_{\ell_{(i)}-1}^{(i)}(\bar{\nu})>x_{\ell}$. We also have $P_{\ell^{(i)}-1}^{(i)}(\bar{\nu}) \geq x_{\ell}$ by Proposition 5.23. Thus $\tilde{f}_{i}$ acts on the same string before and after the application of $\delta$. We notice that $\delta$ acts on the same string before and after the application of $\widetilde{f}_{i}$ even if $\widetilde{f}_{i}$ creates a singular string since we are assuming 
that $\ell_{(i+1)}=\ell_{(i)}$. Here recall that $\tilde{\ell}_{(i)}$ is determined as the length of the minimal possible string compared with the length $\tilde{\ell}_{(i+1)}=\ell_{(i+1)}$ and the length $\ell_{(i)}$ singular string remains singular after the application of $\tilde{f}_{i}$.

Proof of (2) is the same as Proposition 5.24.

Proposition 5.26. Assume that $\ell=\ell^{(i)} \leq \ell^{(i+1)}<\ell_{(i+1)}=\ell_{(i)}, m_{\ell}^{(i)}(\nu)>1$ and $\ell=j$. Then we have the following identities:

$$
\text { (1) } \quad \overline{\widetilde{\nu}}=\widetilde{\bar{\nu}}, \quad(2) \quad \overline{\widetilde{J}}=\widetilde{\bar{J}} .
$$

Proof. (1) We divide the proof into two cases according to whether $\ell=\ell_{(i)}-1$ or $\ell<\ell_{(i)}-1$.

Case 1. Let us consider the case $\ell=\ell_{(i)}-1$. Then we have at least three strings $\left(\ell, x_{\ell}\right)$, $\left(\ell, P_{\ell}^{(i)}(\nu)\right)$ and $\left(\ell+1, P_{\ell+1}^{(i)}(\nu)\right)$ in $(\nu, J)^{(i)}$. Let us analyze the action of $\tilde{f}_{i}$ before and after the application of $\delta$. In this case, we show $P_{\ell_{(i)}-1}^{(i)}(\bar{\nu}) \geq x_{\ell}$. According to Lemma 5.1 (VI) we have to show $P_{\ell_{(i)}-1}^{(i)}(\nu) \geq x_{\ell}$, which is the consequence of $\ell=\ell_{(i)}-1$. Recall also that $P_{\ell-1}^{(i)}(\bar{\nu}) \geq x_{\ell}$ by Proposition 5.23 .

If $P_{\ell}^{(i)}(\bar{\nu})>x_{\ell}$ there is no problem since the string $\left(\ell, x_{\ell}\right)$ of $(\bar{\nu}, \bar{J})^{(i)}$ becomes non-singular. So suppose that $P_{\ell}^{(i)}(\bar{\nu})=x_{\ell}$. Then there are three strings $\left(\ell-1, P_{\ell-1}^{(i)}(\bar{\nu})\right),\left(\ell, x_{\ell}\right)$, and $\left(\ell, x_{\ell}\right)$ in $(\bar{\nu}, \bar{J})^{(i)}$. Thus we can choose the strings such that $\widetilde{f}_{i}$ acts on the same string before and after the application of $\delta$. Also, since we are assuming that $\ell_{(i+1)}=\ell_{(i)}, \delta$ acts on the same string before and after the application of $\tilde{f}_{i}$ even if $\tilde{f}_{i}$ creates a singular string. Thus we have $\overline{\widetilde{\nu}}=\widetilde{\bar{\nu}}$ in this case.

Case 2. Let us consider the case $\ell<\ell_{(i)}-1$. In this case, we can use the same arguments of Case (VI) of the proof of Proposition 5.8 to show $P_{\ell_{(i)}-1}^{(i)}(\bar{\nu})>x_{\ell}$. Thus we see that $\tilde{f}_{i}$ acts on the same string before and after the application of $\delta$. Also, by the assumption $\ell_{(i+1)}=\ell_{(i)}$, we see that $\delta$ acts on the same string before and after the application of $\widetilde{f}_{i}$. Thus we have $\overline{\widetilde{\nu}}=\widetilde{\bar{\nu}}$ in this case.

Proof of (2) is the same as Proposition 5.24.

Proposition 5.27. Assume that $\ell=\ell^{(i)} \leq \ell_{(i+1)}<\ell_{(i)}, m_{\ell}^{(i)}(\nu)>1$ and $\ell=j$. Then we have the following identities:

$$
\text { (1) } \quad \overline{\widetilde{\nu}}=\widetilde{\bar{\nu}}, \quad(2) \quad \overline{\widetilde{J}}=\widetilde{\bar{J}} .
$$

Proof. Step 1. Suppose that we have $P_{\ell_{(i)}}^{(i)}(\bar{\nu})>x_{\ell}$. Since we have $P_{\ell-1}^{(i)}(\bar{\nu}) \geq x_{\ell}$ by Proposition 5.23, we see that $\widetilde{f}_{i}$ acts on the same string before and after $\delta$. Let us consider the behavior of $\delta$ before and after $\widetilde{f}_{i}$. Then a subtlety could occur only if $\widetilde{f}_{i}$ creates a singular string which satisfies $\ell_{(i)}>\ell+1 \geq \ell_{(i+1)}$.

Thus suppose if possible that $P_{\ell_{(i)}-1}^{(i)}(\bar{\nu})>x_{\ell}, \widetilde{f}_{i}$ creates a singular string of length $\ell+1$ and $\ell_{(i)}>\ell+1 \geq \ell_{(i+1)}$. From Lemma 5.1 (VII) we have $P_{\ell_{(i)}-1}^{(i)}(\nu)>x_{\ell}+1$ and from Lemma 5.2 we have $P_{\ell+1}^{(i)}(\nu)=x_{\ell}+1$. If $\ell_{(i)}-1=\ell+1$ this is a contradiction. Thus suppose that $\ell_{(i)}-1>\ell+1$. Recall that we have $P_{\ell}^{(i)}(\nu) \geq x_{\ell}$ by definition of the rigged configurations. Suppose if possible that $P_{\ell}^{(i)}(\nu)>x_{\ell}$. Then we have $P_{\ell}^{(i)}(\nu) \geq P_{\ell+1}^{(i)}(\nu)<P_{\ell_{(i)}-1}^{(i)}(\nu)$ which violates the convexity relation of $P_{k}^{(i)}(\nu)$ between $\ell \leq k \leq \ell_{(i)}$. Thus we have $P_{\ell}^{(i)}(\nu)=x_{\ell}$. Suppose if possible that $\ell_{(i+1)}=\ell$. Since $P_{\ell}^{(i)}(\nu)=x_{\ell}$ we see that all length $\ell$ strings of $(\nu, J)^{(i)}$ are singular. This is 
a contradiction since we have $m_{\ell}^{(i)}(\nu)>1$ and $\ell_{(i)}>\ell_{(i+1)}$. Thus we have $\ell_{(i+1)}=\ell+1$. From $P_{\ell+1}^{(i)}(\nu)=x_{\ell}+1$ and $P_{\ell_{(i)}-1}^{(i)}(\nu)>x_{\ell}+1$ we have

$$
P_{\ell+2}^{(i)}(\nu) \geq \min \left\{P_{\ell+1}^{(i)}(\nu), P_{\ell_{(i)}-1}^{(i)}(\nu)\right\}=x_{\ell}+1 .
$$

Let us write $P_{\ell+2}^{(i)}(\nu)=x_{\ell}+1+\varepsilon$ where $\varepsilon \geq 0$. From Lemma 3.13 with $l=\ell+1$, we have

$$
\begin{aligned}
& -P_{\ell}^{(i)}(\nu)+2 P_{\ell+1}^{(i)}(\nu)-P_{\ell+2}^{(i)}(\nu)=1-\varepsilon \\
& \quad \geq m_{\ell+1}^{(i-1)}(\nu)-2 m_{\ell+1}^{(i)}(\nu)+m_{\ell+1}^{(i+1)}(\nu)=m_{\ell+1}^{(i-1)}(\nu)+m_{\ell+1}^{(i+1)}(\nu) .
\end{aligned}
$$

Since we have $m_{\ell+1}^{(i+1)}(\nu)>0$ by $\ell_{(i+1)}=\ell+1$, the only possibility is $\varepsilon=0, m_{\ell+1}^{(i-1)}(\nu)=0$ and $m_{\ell+1}^{(i+1)}(\nu)=1$. In particular, we have $P_{\ell+2}^{(i)}(\nu)=x_{\ell}+1$. However this is a contradiction since we have $P_{\ell+1}^{(i)}(\nu)=P_{\ell+2}^{(i)}(\nu)<P_{\ell_{(i)}-1}^{(i)}(\nu)$ which violates the convexity relation of $P_{k}^{(i)}(\nu)$ between $\ell \leq k \leq \ell_{(i)}$. Hence this case cannot happen. Therefore we have $\overline{\widetilde{\nu}}=\widetilde{\bar{\nu}}$ and $\overline{\widetilde{J}}=\widetilde{\bar{J}}$ in this case.

Step 2. Suppose that we have $\ell=\ell_{(i)}-1$. By definition of the rigged configuration we have $P_{\ell}^{(i)}(\nu) \geq x_{\ell}$. Then by Lemma 5.1 (VII) we have $P_{\ell}^{(i)}(\bar{\nu}) \geq x_{\ell}-1$. Suppose if possible that $P_{\ell}^{(i)}(\bar{\nu})=x_{\ell}-1$. Recall that we have the three strings $\left(\ell, x_{\ell}\right),\left(\ell, P_{\ell}^{(i)}(\nu)\right)$ and $\left(\ell+1, P_{\ell+1}^{(i)}(\nu)\right)$ of $(\nu, J)^{(i)}$. Then we have the remaining string $\left(\ell, x_{\ell}\right)$ in $(\bar{\nu}, \bar{J})^{(i)}$. This is a contradiction since we have $P_{\ell}^{(i)}(\bar{\nu})<x_{\ell}$.

Thus suppose that $P_{\ell}^{(i)}(\bar{\nu}) \geq x_{\ell}$. As in Case 1 of the proof of the previous proposition we can show that $\tilde{f}_{i}$ chooses the same string before and after $\delta$. Let us consider the behavior of $\delta$ before and after $\tilde{f}_{i}$. In this case, there is no problem even if $\widetilde{f}_{i}$ creates a singular string since we have $\ell+1=\ell_{(i)}$. Hence we have $\overline{\widetilde{\nu}}=\widetilde{\bar{\nu}}$ and $\widetilde{\widetilde{J}}=\widetilde{\bar{J}}$.

Step 3. We assume that $\ell<\ell_{(i)}-1$ and $P_{\ell_{(i)}}^{(i)}(\bar{\nu}) \leq x_{\ell}$ in the following proof. According to Lemma 5.1 (VII) we have $P_{\ell_{(i)}-1}^{(i)}(\nu) \leq x_{\ell}+1$. Recall that we have $P_{\ell_{(i)}}^{(i)}(\nu)>x_{\ell}$ by $\ell_{(i)}>\ell$ and $P_{\ell}^{(i)}(\nu) \geq x_{\ell}$ by definition of the rigged configurations. Since we are assuming that $\ell=$ $\ell^{(i)}=j<\ell_{(i)}-1$, the convexity relation of the vacancy numbers allows the following three possibilities:

(1) $\ell=\ell_{(i)}-2, P_{\ell}^{(i)}(\nu)=x_{\ell}, P_{\ell_{(i)}}^{(i)}(\nu)=x_{\ell}+1$ and $P_{\ell_{(i)}}^{(i)}(\nu)=x_{\ell}+2$,

(2) $P_{\ell}^{(i)}(\nu)=x_{\ell}$ and $P_{\ell+1}^{(i)}(\nu)=\cdots=P_{\ell_{(i)}}^{(i)}(\nu)=x_{\ell}+1$,

(3) $P_{\ell}^{(i)}(\nu)=P_{\ell+1}^{(i)}(\nu)=\cdots=P_{\ell_{(i)}}^{(i)}(\nu)=x_{\ell}+1$.

In the following we consider them case by case.

Case (1). According to Lemma 3.13 with $l=\ell_{(i)}-1$, we have

$$
\begin{aligned}
& -P_{\ell}^{(i)}(\nu)+2 P_{\ell_{(i)}-1}^{(i)}(\nu)-P_{\ell_{(i)}}^{(i)}(\nu)=0 \\
& \quad \geq m_{\ell_{(i)}-1}^{(i-1)}(\nu)-2 m_{\ell_{(i)}-1}^{(i)}(\nu)+m_{\ell_{(i)}-1}^{(i+1)}(\nu)=m_{\ell_{(i)}-1}^{(i-1)}(\nu)+m_{\ell_{(i)}-1}^{(i+1)}(\nu)
\end{aligned}
$$

where we have used $m_{\ell_{(i)}-1}^{(i)}(\nu)=0$ by $j=\ell$. Thus we have $m_{\ell_{(i)}-1}^{(i-1)}(\nu)=m_{\ell_{(i)}-1}^{(i+1)}(\nu)=0$. Since we are assuming that $\ell_{(i+1)}<\ell_{(i)}$, we conclude that $\ell^{(i+1)}=\ell_{(i+1)}=\ell$.

Now recall that we are assuming that $m_{\ell}^{(i)}(\nu) \geq 2$. Then the minimality of $x_{\ell}$ and the assumption $P_{\ell}^{(i)}(\nu)=x_{\ell}$ imply that all the length $\ell$ strings of $(\nu, J)^{(i)}$ are $\left(\ell, P_{\ell}^{(i)}(\nu)\right)$, in particular, 
they are singular. However this is in contradiction to the assumption $\ell_{(i+1)}<\ell_{(i)}$ since we know that $\ell_{(i+1)}=\ell$ which forces that $\ell_{(i)}=\ell$. Thus this case cannot happen.

Case (2). The assumption $P_{\ell+1}^{(i)}(\nu)=\cdots=P_{\ell_{(i)}}^{(i)}(\nu)$ implies that $m_{k}^{(i-1)}(\nu)=m_{k}^{(i+1)}(\nu)=0$ for all $\ell+2 \leq k \leq \ell_{(i)}-1$ as otherwise the above relation for the vacancy numbers would be strictly convex. Next, we apply Lemma 3.13 with $l=\ell+1$. Then we have

$$
\begin{aligned}
& -P_{\ell}^{(i)}(\nu)+2 P_{\ell+1}^{(i)}(\nu)-P_{\ell+2}^{(i)}(\nu)=1 \\
& \quad \geq m_{\ell+1}^{(i-1)}(\nu)-2 m_{\ell+1}^{(i)}(\nu)+m_{\ell+1}^{(i+1)}(\nu)=m_{\ell+1}^{(i-1)}(\nu)+m_{\ell+1}^{(i+1)}(\nu),
\end{aligned}
$$

where we have used $m_{\ell+1}^{(i)}(\nu)=0$ by $j=\ell$.

Suppose if possible that $m_{\ell+1}^{(i-1)}(\nu)=1$. Then we have $m_{\ell+1}^{(i+1)}(\nu)=0$. Since we are assuming that $\ell_{(i+1)}<\ell_{(i)}, m_{k}^{(i+1)}(\nu)=0$ for all $\ell+1 \leq k \leq \ell_{(i)}-1$ implies that $\ell_{(i+1)}=\ell$. However, as in the previous case, the assumption $P_{\ell}^{(i)}(\nu)=x_{\ell}$ implies that all the length $\ell$ strings of $(\nu, J)^{(i)}$ are singular. Thus we must have $\ell_{(i)}=\ell$ by $\ell_{(i+1)}=\ell$ and $m_{\ell}^{(i)}(\nu)>1$. This is a contradiction. Hence this case cannot happen.

Therefore we assume that $m_{\ell+1}^{(i-1)}(\nu)=0$ and $m_{\ell+1}^{(i+1)}(\nu)=1$ in the sequel. Then we can show that $\ell^{(i+1)}=\ell$ and $\ell_{(i+1)}=\ell+1$. Since $m_{k}^{(i+1)}(\nu)=0$ for all $\ell+2 \leq k \leq \ell_{(i)}-1$, we have $\ell_{(i+1)}=\ell$ or $\ell+1$ by $\ell \leq \ell_{(i+1)}$. Suppose if possible that $\ell_{(i+1)}=\ell$. Since we are assuming that $P_{\ell}^{(i)}(\nu)=x_{\ell}$, the length $\ell=\ell^{(i)}$ strings of $(\nu, J)^{(i)}$ are always equal to $\left(\ell, x_{\ell}\right)$ by the minimality of $x_{\ell}$. Then by the assumption $m_{\ell}^{(i)}(\nu) \geq 2$, we must have $\ell_{(i)}=\ell$ by $\ell_{(i+1)}=\ell$. This is in contradiction to the other assumption $\ell^{(i)}<\ell_{(i)}$. Thus we have $\ell_{(i+1)}=\ell+1$. Then from $m_{\ell+1}^{(i+1)}(\nu)=1$, we conclude that $\ell^{(i+1)}=\ell$ since there is the requirement $\ell \leq \ell^{(i+1)} \leq \ell_{(i+1)}$.

Let us analyze $\widetilde{f}_{i} \circ \delta$ first. Since $\widetilde{f}_{i}$ acts on the $i$-th place, we have $\widetilde{\bar{\nu}}^{(a)}=\bar{\nu}^{(a)}$ for all $a \neq i$. Next we consider $\widetilde{\widetilde{\nu}}^{(i)}$. Recall that we have $P_{\ell^{(i)}-1}^{(i)}(\bar{\nu}) \geq x_{\ell}$ by Proposition 5.23. Also, since we have $P_{\ell_{(i)}-1}^{(i)}(\nu)=x_{\ell}+1$, we have $P_{\ell_{(i)}-1}^{(i)}(\bar{\nu})=x_{\ell}$ by Lemma 5.1 (VII). Therefore the string $\left(\ell_{(i)}-1, P_{\ell_{(i)}-1}^{(i)}(\bar{\nu})\right)$ of $(\bar{\nu}, \bar{J})^{(i)}$ has the largest length among the strings with smallest rigging, hence $\widetilde{f}_{i}$ acts on it. To summarize, $\widetilde{\bar{\nu}}^{(i)}$ is obtained from $\nu^{(i)}$ by removing one box from a length $\ell^{(i)}$ row.

Next let us analyze $\delta \circ \widetilde{f}_{i}$. Since $\widetilde{f}_{i}$ does not change the coriggings of untouched strings, and since we are assuming that $m_{\ell}^{(i)}(\nu) \geq 2$, we have $\widetilde{\ell}^{(a)}=\ell^{(a)}$ for all $a$ and $\widetilde{\ell}_{(a)}=\ell_{(a)}$ for all $a \geq i+1$. Let us consider $\tilde{\ell}_{(i)}$. Recall that we have $\tilde{\ell}_{(i+1)}=\ell_{(i+1)}=\ell+1$ in this case. Since we have $P_{\ell+1}^{(i)}(\nu)=x_{\ell}+1, \widetilde{f}_{i}$ creates a singular string of length $\ell+1$ by Lemma 5.2 . Thus the length $\ell+1$ string created by $\widetilde{f}_{i}$ is the shortest possible string starting from $\tilde{\ell}_{(i+1)}$. Therefore we have $\tilde{\ell}_{(i)}=\ell+1$. Since we have $m_{k}^{(i-1)}(\nu)=0$ for all $\ell+1 \leq k \leq \ell_{(i)}-1$ and since $\tilde{f}_{i}$ does not change the coriggings of untouched strings, we have $\widetilde{\ell}_{(i-1)}=\ell_{(i-1)}$ and thus $\widetilde{\ell}_{(a)}=\ell_{(a)}$ for all $a \leq i-1$. To summarize, $\overline{\widetilde{\nu}}^{(i)}$ is obtained from $\nu^{(i)}$ by removing one box from a length $\ell^{(i)}$ row and $\overline{\widetilde{\nu}}^{(a)}=\bar{\nu}^{(a)}$ for all $a \neq i$. Hence we have $\overline{\widetilde{\nu}}=\widetilde{\bar{\nu}}$.

Finally, let us show $\overline{\widetilde{J}}=\widetilde{\bar{J}}$. For this it is enough to check the three strings $\left(\ell^{(i)}, P_{\ell^{(i)}}^{(i)}(\nu)\right)$, $\left(\ell, x_{\ell}\right)$ and $\left(\ell_{(i)}, P_{\ell_{(i)}}^{(i)}(\nu)\right)$ of $(\nu, J)^{(i)}$. Here note that we have in fact $\left(\ell^{(i)}, P_{\ell^{(i)}}^{(i)}(\nu)\right)=\left(\ell, x_{\ell}\right)$ under the present assumption. To begin with, the string $\left(\ell^{(i)}, P_{\ell^{(i)}}^{(i)}(\nu)\right)$ behaves as follows:

$$
\begin{array}{lllll}
\left(\ell^{(i)}, P_{\ell^{(i)}}^{(i)}(\nu)\right) & \stackrel{\tilde{f}_{i}}{\longmapsto}\left(\ell^{(i)}, P_{\ell^{(i)}}^{(i)}(\widetilde{\nu})\right) & \stackrel{\delta}{\longmapsto} & \left(\ell^{(i)}-1, P_{\ell^{(i)}-1}^{(i)}(\overline{\widetilde{\nu}})\right), \\
\left(\ell^{(i)}, P_{\ell^{(i)}}^{(i)}(\nu)\right) & \stackrel{\delta}{\longmapsto}\left(\ell^{(i)}-1, P_{\ell^{(i)}-1}^{(i)}(\bar{\nu})\right) & \widetilde{f}_{i} & \left(\ell^{(i)}-1, P_{\ell^{(i)}-1}^{(i)}(\widetilde{\bar{\nu}})\right) .
\end{array}
$$


By $\overline{\widetilde{\nu}}=\widetilde{\bar{\nu}}$ we see the coincidence of the riggings. Next, let us consider the string $\left(\ell, x_{\ell}\right)$ :

$$
\begin{aligned}
& \left(\ell, x_{\ell}\right) \stackrel{\widetilde{f}_{i}}{\longmapsto}\left(\ell+1, x_{\ell}-1\right) \stackrel{\delta}{\stackrel{\delta}{\longmapsto}}\left(\ell, P_{\ell}^{(i)}(\overline{\widetilde{\nu}})\right), \\
& \left(\ell, x_{\ell}\right) \stackrel{\delta}{\longmapsto}\left(\ell, x_{\ell}\right) \quad \stackrel{\widetilde{f}_{i}}{\longmapsto}\left(\ell, x_{\ell}\right) .
\end{aligned}
$$

Recall that we have $\widetilde{\ell}^{(i-1)} \leq \widetilde{\ell}^{(i)}=\widetilde{\ell}^{(i+1)}=\ell$ and $\ell+1=\widetilde{\ell}_{(i+1)}=\widetilde{\ell}_{(i)}<\widetilde{\ell}_{(i-1)}$. Therefore we have $P_{\ell}^{(i)}(\overline{\widetilde{\nu}})=P_{\ell}^{(i)}(\nu)=x_{\ell}$. Let us consider the remaining string $\left(\ell_{(i)}, P_{\ell_{(i)}}^{(i)}(\nu)\right)$. Then we have

$$
\begin{array}{llll}
\left(\ell_{(i)}, P_{\ell_{(i)}}^{(i)}(\nu)\right) & \stackrel{\widetilde{f}_{i}}{\longmapsto}\left(\ell_{(i)}, P_{\ell_{(i)}^{(i)}}^{(\widetilde{\nu}))}\right. & \stackrel{\delta}{\longmapsto}\left(\ell_{(i)}, P_{\ell_{(i)}}^{(i)}(\widetilde{\nu})\right), \\
\left(\ell_{(i)}, P_{\ell_{(i)}}^{(i)}(\nu)\right) & \stackrel{\delta}{\longmapsto}\left(\ell_{(i)}-1, P_{\ell_{(i)}-1}^{(i)}(\bar{\nu})\right) & \widetilde{f}_{i} & \left(\ell_{(i)}, P_{\ell_{(i)}}^{(i)}(\bar{\nu})-1\right) .
\end{array}
$$

Since we have $\ell+1 \leq \ell_{(i)}$ by the assumption, we have $P_{\ell_{(i)}}^{(i)}(\widetilde{\nu})=P_{\ell_{(i)}}^{(i)}(\nu)-2=x_{\ell}-1$. Also, since we are assuming that $\ell_{(i+1)}<\ell_{(i)}$, we have $P_{\ell_{(i)}-1}^{(i)}(\bar{\nu})=P_{\ell_{(i)}-1}^{(i)}(\nu)-1$ by Lemma 5.1 (VII). Thus we have $P_{\ell_{(i)}-1}^{(i)}(\bar{\nu})-1=P_{\ell_{(i)}}^{(i)}(\nu)-2=x_{\ell}-1$. Therefore we have checked $\overline{\widetilde{J}}=\widetilde{\bar{J}}$ in this case.

Case (3). The assumption $P_{\ell}^{(i)}(\nu)=\cdots=P_{\ell_{(i)}}^{(i)}(\nu)$ implies that $m_{k}^{(i-1)}(\nu)=m_{k}^{(i+1)}(\nu)=0$ for all $\ell+1 \leq k \leq \ell_{(i)}-1$. Since we are assuming that $\ell_{(i+1)}<\ell_{(i)}$, the only possibility is $\ell_{(i+1)}=\ell$.

Let us analyze $\tilde{f}_{i} \circ \delta$ first. From Lemma 5.1 (VII) we have $P_{\ell_{(i)}-1}^{(i)}(\bar{\nu})=x_{\ell}$ by $P_{\ell_{(i)}-1}^{(i)}(\nu)=x_{\ell}+1$. Recall that we have $P_{\ell^{(i)}-1}^{(i)}(\bar{\nu}) \geq x_{\ell}$ by Proposition 5.23. From the assumption $\ell \leq \ell_{(i)}-1$, we see that the string $\left(\ell_{(i)}-1, P_{\ell_{(i)}-1}^{(i)}(\bar{\nu})\right)$ has the smallest rigging of the largest length. Thus the next $\widetilde{f}_{i}$ acts on this string. To summarize, we have $\widetilde{\bar{\nu}}^{(a)}=\bar{\nu}^{(a)}$ for all $a \neq i$ and $\widetilde{\bar{\nu}}^{(i)}$ is obtained by removing one box from the length $\ell^{(i)}$ row.

Next we analyze $\delta \circ \widetilde{f}_{i}$. Since $\widetilde{f}_{i}$ does not change coriggings of untouched strings, and since we are assuming that $m_{\ell}^{(i)}(\nu)>1$, we have $\widetilde{\ell}^{(a)}=\ell^{(a)}$ for all $a$ and $\widetilde{\ell}_{(a)}=\ell_{(a)}$ for all $a \geq i+1$. In particular, we have $\tilde{\ell}_{(i+1)}=\ell_{(i+1)}=\ell$ by the assumption. In order to determine $\tilde{\ell}_{(i)}$, recall that the relation $P_{\ell+1}^{(i)}(\nu)=x_{\ell}+1$ implies that $\widetilde{f}_{i}$ creates the singular string of length $\ell+1$ by Lemma 5.2. Thus we have $\tilde{\ell}_{(i)}=\ell+1$. Since we have $m_{k}^{(i-1)}(\nu)=0$ for all $\ell+1 \leq k \leq \ell_{(i)}-1$, we have $\tilde{\ell}_{(i-1)}=\ell_{(i-1)}$ by $\ell_{(i-1)} \geq \ell_{(i)}$, thus $\tilde{\ell}_{(a)}=\ell_{(a)}$ for all $a \leq i-1$. To summarize, we have $\overline{\widetilde{\nu}}^{(a)}=\bar{\nu}^{(a)}$ for all $a \neq i$ and $\overline{\widetilde{\nu}}^{(i)}$ is obtained by removing one box from the length $\ell^{(i)}$ row. Hence we have $\widetilde{\widetilde{\nu}}=\overline{\widetilde{\nu}}$.

Let us show $\widetilde{\bar{J}}=\tilde{\widetilde{J}}$ in this case. Again it is enough to check the three strings $\left(\ell^{(i)}, P_{\ell^{(i)}}^{(i)}(\nu)\right)$, $\left(\ell, x_{\ell}\right)$ and $\left(\ell_{(i)}, P_{\ell_{(i)}^{(i)}}^{(\nu))}\right.$ of $(\nu, J)^{(i)}$. The analysis for the string $\left(\ell^{(i)}, P_{\ell^{(i)}}^{(i)}(\nu)\right)$ is the same with the previous Case $(2)$. Let us analyze the string $\left(\ell, x_{\ell}\right)$. Then it behaves as

$$
\begin{array}{ll}
\left(\ell, x_{\ell}\right) \stackrel{\widetilde{f}_{i}}{\longmapsto}\left(\ell+1, x_{\ell}-1\right) \stackrel{\delta}{\longmapsto}\left(\ell, P_{\ell}^{(i)}(\overline{\widetilde{\nu}})\right), \\
\left(\ell, x_{\ell}\right) \stackrel{\delta}{\longmapsto}\left(\ell, x_{\ell}\right) & \stackrel{\widetilde{f}_{i}}{\longmapsto}\left(\ell, x_{\ell}\right) .
\end{array}
$$

In this case, we have $\tilde{\ell}^{(i-1)} \leq \widetilde{\ell}^{(i)}=\widetilde{\ell}^{(i+1)}=\ell$ and $\ell=\widetilde{\ell}_{(i+1)}<\tilde{\ell}_{(i)} \leq \tilde{\ell}_{(i-1)}$. Therefore we have $P_{\ell}^{(i)}(\overline{\widetilde{\nu}})=P_{\ell}^{(i)}(\nu)-1=x_{\ell}$. The analysis of $\left(\ell_{(i)}, P_{\ell_{(i)}}^{(i)}(\nu)\right)$ is the same with the previous Case (2). Thus we have $\widetilde{\bar{J}}=\overline{\widetilde{J}}$ in this case. This completes the proof of proposition. 
Example 5.28. Here we give an example for Case (2) of Step 3 of the proof of Proposition 5.27. Consider the following rigged configuration $(\nu, J)$ of type $\left(B^{1,1}\right)^{\otimes 3} \otimes B^{1,3} \otimes B^{2,1} \otimes B^{2,2} \otimes B^{3,1}$ of $D_{5}^{(1)}$
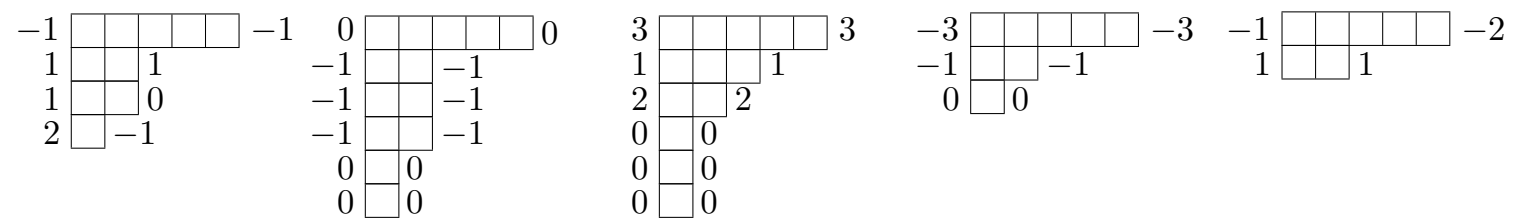

The corresponding tensor product $\Phi^{-1}(\nu, J)$ is

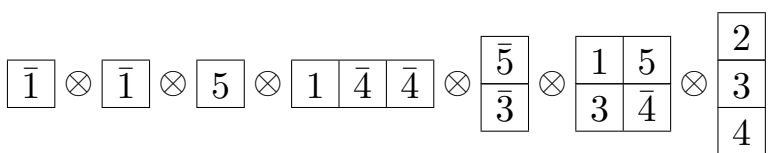

Then $(\bar{\nu}, \bar{J})$ is
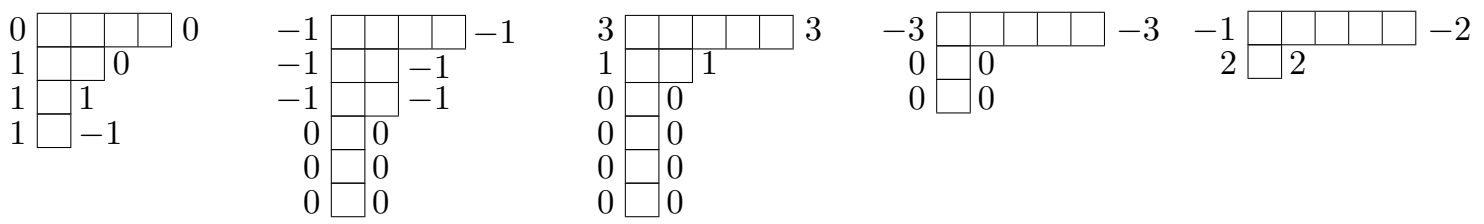

and $(\widetilde{\nu}, \widetilde{J})$ is
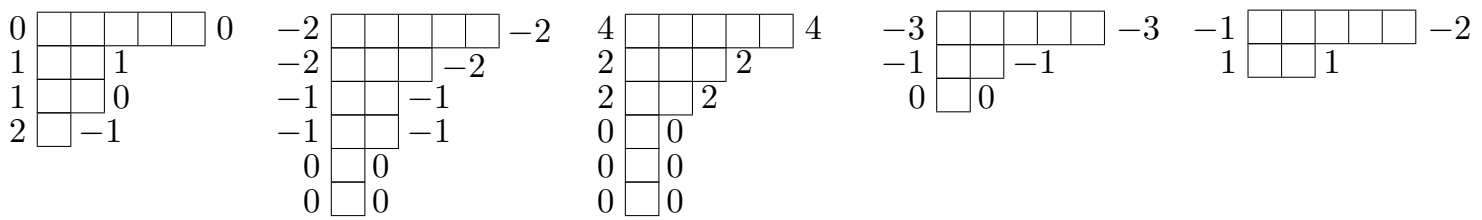

In this example, we have $\ell=\ell^{(2)}=2<\ell_{(3)}=3<\ell_{(2)}=5, m_{2}^{(2)}(\nu)=3$ and $m_{k}^{(2)}(\nu)=0$ for $\ell^{(2)}<k<\ell_{(2)}$. Moreover, we have $\ell<\ell_{(2)}-1$ and $P_{\ell_{(2)}-1}^{(2)}(\bar{\nu})=x_{\ell}$ where $x_{\ell}=-1$. This example satisfies the condition for Case $(2)$

$$
P_{2}^{(2)}(\nu)=x_{\ell}=-1, \quad P_{3}^{(2)}(\nu)=P_{4}^{(2)}(\nu)=P_{5}^{(2)}(\nu)=x_{\ell}+1=0 .
$$

As discussed in the proof, we have $m_{4}^{(1)}(\nu)=m_{4}^{(3)}(\nu)=0$ where $\ell+2=\ell_{(2)}-1=4, m_{3}^{(1)}(\nu)=0$ and $m_{3}^{(3)}(\nu)=1$. Finally $(\overline{\widetilde{\nu}}, \overline{\widetilde{J}})=(\widetilde{\bar{\nu}}, \widetilde{\bar{J}})$ is

\begin{tabular}{|c|c|c|}
\hline-2 & & \\
\hline-1 & & $\begin{array}{c}1 \\
-1\end{array}$ \\
\hline-1 & & -1 \\
\hline 0 & 0 & \\
\hline & 0 & \\
\hline
\end{tabular}

\begin{tabular}{l|l|l|l|}
\cline { 2 - 2 } & & & \\
1 & & & 0 \\
1 & & \\
1 &
\end{tabular}

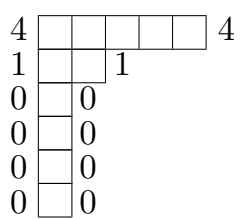

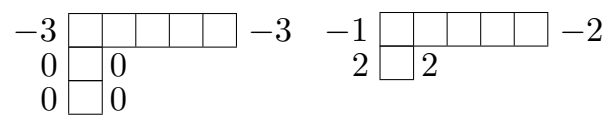

\subsection{Proof for Case D (2)}

Proposition 5.29. Suppose that $\ell=\ell^{(i)}=\ell_{(i)}$ and $m_{\ell}^{(i)}(\nu)>2$. Then we have the following identities:
(1) $\quad \overline{\widetilde{\nu}}=\widetilde{\bar{\nu}}$
(2) $\overline{\widetilde{J}}=\widetilde{\bar{J}}$. 
Proof. By the assumption $m_{\ell}^{(i)}(\nu) \geq 3$ we can choose the three distinct strings

$$
\left(\ell^{(i)}, P_{\ell^{(i)}}^{(i)}(\nu)\right)=\left(\ell_{(i)}, P_{\ell_{(i)}^{(i)}}^{(\nu)}\right) \text { and }\left(\ell, x_{\ell}\right)
$$

of the $i$-th configuration. Here we may have $P_{\ell^{(i)}}^{(i)}(\nu)=x_{\ell}$.

Let us analyze $\widetilde{f}_{i} \circ \delta$. After the application of $\delta$ the above three strings become

$$
\left(\ell^{(i)}-1, P_{\ell^{(i)}-1}^{(i)}(\bar{\nu})\right)=\left(\ell_{(i)}-1, P_{\ell_{(i)}-1}^{(i)}(\bar{\nu})\right) \text { and }\left(\ell, x_{\ell}\right) .
$$

By Proposition 5.23 we have $P_{\ell^{(i)}-1}^{(i)}(\bar{\nu})=P_{\ell_{(i)}-1}^{(i)}(\bar{\nu}) \geq x_{\ell}$. Thus the string $\left(\ell, x_{\ell}\right)$ remains as the string with smallest rigging of the largest length. Hence $\widetilde{f}_{i}$ will act on it. To summarize $\widetilde{\bar{\nu}}^{(a)}=\bar{\nu}^{(a)}$ for all $a \neq i$ and $\widetilde{\bar{\nu}}^{(i)}$ is obtained by removing a box from each of the two length $\ell^{(i)}=\ell_{(i)}$ rows and adding a box to the length $\ell$ row.

Let us analyze $\delta \circ \tilde{f}_{i}$. Since $\tilde{f}_{i}$ does not change coriggings of untouched strings, we have $\widetilde{\ell}^{(a)}=\ell^{(a)}$ for all $a$ and $\tilde{\ell}_{(a)}=\ell_{(a)}$ for all $a \geq i+1$. Since the string $\left(\ell_{(i)}, P_{\ell_{(i)}}^{(i)}(\nu)\right)$ remains as the singular string which is longer than $\tilde{\ell}_{(i+1)}$, we have $\tilde{\ell}_{(i)}=\ell_{(i)}$ and hence $\widetilde{\ell}_{(a)}=\ell_{(a)}$ for all $a \leq i$. Thus we obtain $\overline{\widetilde{\nu}}=\widetilde{\bar{\nu}}$. The remaining statement $\overline{\widetilde{J}}=\widetilde{\bar{J}}$ follows from $\overline{\widetilde{\nu}}=\widetilde{\bar{\nu}}$.

\subsection{Proof for Case D (3)}

\subsubsection{Outline}

Let us consider the third case. We prove the following properties in the latter part of this subsection by case by case analysis.

Proposition 5.30. Assume that $\ell^{(i)}=\ell$ and $m_{\ell}^{(i)}(\nu)=1$. Then the following relations hold:

$$
\begin{aligned}
& P_{\ell+1}^{(i)}(\nu)=x_{\ell}+1, \\
& P_{\ell-1}^{(i)}(\bar{\nu})=x_{\ell}, \\
& \ell+1 \leq \ell^{(i+1)} .
\end{aligned}
$$

Next let us show the following property:

Proposition 5.31. Assume that $\ell=\ell^{(i)}<\ell_{(i)}$ and $m_{\ell}^{(i)}(\nu)=1$. Then the following relation holds:

$$
P_{\ell_{(i)}-1}^{(i)}(\bar{\nu})>x_{\ell}
$$

Proof. We follow the classification of Lemma 5.1. Since we are assuming that $\ell^{(i)}<\ell_{(i)}$, we have to deal with Cases (V), (VI) and (VII). Throughout the proof of this proposition, let $j$ be the largest integer such that $j<\ell_{(i)}$ and $m_{j}^{(i)}(\nu)>0$. Since we are assuming that $\ell^{(i)}<\ell_{(i)}$, we have $\ell^{(i)} \leq j$.

Case $(\mathbf{V})$. In this case, we have to show that $P_{\ell_{(i)}}^{(i)}(\nu) \geq x_{\ell}$ under the assumption $\ell^{(i)}<$ $\ell^{(i+1)}=\cdots=\ell_{(i)}$. We can use the same argument of the corresponding part of Proposition 5.8 to show the assertion.

Case (VI). In this case, we have to show that $P_{\ell_{(i)}-1}^{(i)}(\nu)>x_{\ell}$ under the assumption $\ell^{(i+1)}<$ $\ell_{(i+1)}=\ell_{(i)}$. Suppose if possible that $P_{\ell_{(i)}-1}^{(i)}(\nu) \leq x_{\ell}$. 
Let us consider the case $j=\ell_{(i)}-1$. Note that by (5.16), which is shown independently to the present proposition, we have $\ell<\ell^{(i+1)}$. Combining with the current assumption $\ell^{(i+1)}<\ell_{(i)}$ we see that $\ell=\ell^{(i)}<\ell_{(i)}-1$. Thus the string $\left(\ell_{(i)}-1, x_{\ell_{(i)}-1}\right)$ of $(\nu, J)^{(i)}$ is different from the string $\left(\ell, x_{\ell}\right)$. Then we have $x_{\ell_{(i)}-1} \leq P_{\ell_{(i)}-1}^{(i)}(\nu) \leq x_{\ell}$. However this is in contradiction to the relation $x_{\ell_{(i)}-1}>x_{\ell}$ which follows from the minimality of $x_{\ell}$ under the condition $\ell_{(i)}-1>\ell$.

Next let us consider the case $j<\ell_{(i)}-1$. Since $\ell^{(i)}<\ell_{(i)}$ we have $P_{\ell_{(i)}}^{(i)}(\nu) \geq x_{\ell_{(i)}}>x_{\ell}$ by the minimality of $x_{\ell}$. Combining with the assumption $P_{\ell_{(i)}-1}^{(i)}(\nu) \leq x_{\ell}$ we have $P_{\ell_{(i)}-1}^{(i)}(\nu)<P_{\ell_{(i)}}^{(i)}(\nu)$. Then from the convexity relation of $P_{k}^{(i)}(\nu)$ between $j \leq k \leq \ell_{(i)}$, we have $x_{j} \leq P_{j}^{(i)}(\nu)<$ $P_{\ell_{(i)}-1}^{(i)}(\nu) \leq x_{\ell}$. This is in contradiction to the relation $x_{j} \geq x_{\ell}$ that follows from the minimality of $x_{\ell}$.

Case (VII). In this case, we have to show that $P_{\ell_{(i)}-1}^{(i)}(\nu)>x_{\ell}+1$ under the assumption $\ell_{(i+1)}<\ell_{(i)}$. Suppose if possible that $P_{\ell_{(i)}-1}^{(i)}(\nu) \leq x_{\ell}+1$. Again recall that we have $\ell<\ell^{(i+1)}$ from (5.16) which is shown independently to the present proposition. Since we have $\ell^{(i+1)} \leq \ell_{(i+1)}$ by definition, we have the relations $\ell=\ell^{(i)}<\ell_{(i+1)}<\ell_{(i)}$.

We divide the proof of Case (VII) into three steps according to the position of $j$.

Step 1. First, let us consider the case $j=\ell_{(i)}-1$. Then there is the corresponding string $\left(\ell_{(i)}-1, x_{\ell_{(i)}-1}\right)$. Since $\ell<\ell_{(i)}-1$, we have $x_{\ell}<x_{\ell_{(i)}-1} \leq P_{\ell_{(i)}-1}^{(i)}(\nu)$ by the minimality of $x_{\ell}$. Combining with the assumption $P_{\ell_{(i)}-1}^{(i)}(\nu) \leq x_{\ell}+1$ we conclude that $x_{\ell_{(i)}-1}=P_{\ell_{(i)}-1}^{(i)}(\nu)$. Then the string $\left(\ell_{(i)}-1, x_{\ell_{(i)}-1}\right)$ is singular and its length satisfies $\ell_{(i+1)} \leq \ell_{(i)}-1<\ell_{(i)}$. This contradicts the definition of $\ell_{(i)}$.

Step 2. Next, let us consider the case $\ell=\ell^{(i)}<j<\ell_{(i)}-1$. Since we are assuming that $\ell^{(i)}<\ell_{(i)}$ we have $P_{\ell_{(i)}}^{(i)}(\nu) \geq x_{\ell_{(i)}}>x_{\ell}$ by the minimality of $x_{\ell}$. Similarly, from $\ell<j$, we have $P_{j}^{(i)}(\nu)>x_{\ell}$. Then, by the convexity of $P_{k}^{(i)}(\nu)$ between $j \leq k \leq \ell_{(i)}$, the only possibility that is compatible with the assumption $P_{\ell_{(i)}-1}^{(i)}(\nu) \leq x_{\ell}+1$ is the situation $P_{j}^{(i)}(\nu)=\cdots=P_{\ell_{(i)}}^{(i)}(\nu)=$ $x_{\ell}+1$.

1. Consider the case $\ell_{(i+1)} \leq j$. Then, combining with the relation $P_{j}^{(i)}(\nu) \geq x_{j}>x_{\ell}$, we obtain $P_{j}^{(i)}(\nu)=x_{j}$. In particular, the string $\left(j, x_{j}\right)$ is singular and its length satisfies that $\ell_{(i+1)} \leq j<\ell_{(i)}$. This contradicts the definition of $\ell_{(i)}$.

2. Consider the case $j<\ell_{(i+1)}$. Since we have $m_{\ell_{(i+1)}}^{(i+1)}(\nu)>0$, the relation for $P_{k}^{(i)}(\nu)$ between $\ell_{(i+1)}-1 \leq k \leq \ell_{(i+1)}+1$ must be strictly convex. This contradicts the above relation.

Step 3. Finally, let us consider the case $\ell=\ell^{(i)}=j$. Since we are assuming that $m_{\ell}^{(i)}(\nu)=1$, there is the only one singular string $\left(\ell, x_{\ell}\right)$. In particular, we have $P_{\ell}^{(i)}(\nu)=x_{\ell}$. Recall that we have $P_{\ell+1}^{(i)}(\nu)=x_{\ell}+1$ by (5.14) which is shown independently to the present proposition. Then the convexity relation of $P_{k}^{(i)}(\nu)$ between $j \leq k \leq \ell_{(i)}$ under the condition $P_{\ell_{(i)}}^{(i)}(\nu)>x_{\ell}$ admits the following two possibilities:

1. The case $\ell^{(i)}+2=\ell_{(i)}, P_{\ell}^{(i)}(\nu)=x_{\ell}, P_{\ell+1}^{(i)}(\nu)=x_{\ell}+1$ and $P_{\ell_{(i)}}^{(i)}(\nu)=x_{\ell}+2$. Since the relation for the vacancy numbers is linear, this situation can happen only if $m_{\ell+1}^{(i+1)}(\nu)=0$. Recall that the relation $\ell=\ell^{(i)}<\ell_{(i+1)}<\ell_{(i)}$ in this case implies that $\ell_{(i+1)}=\ell+1$, in particular, $m_{\ell+1}^{(i+1)}(\nu)>0$. This is a contradiction. 
2. The case $P_{\ell}^{(i)}(\nu)=x_{\ell}$ and $P_{\ell+1}^{(i)}(\nu)=\cdots=P_{\ell_{(i)}}^{(i)}(\nu)=x_{\ell}+1$. Since the relation for $P_{k}^{(i)}(\nu)$ between $\ell+1 \leq k \leq \ell_{(i)}$ is linear, we have $m_{k}^{(i+1)}(\nu)=0$ for all $\ell+1<k<\ell_{(i)}$. Next, applying Lemma 3.13 with $l=\ell+1$ we have

$$
\begin{aligned}
& -P_{\ell}^{(i)}(\nu)+2 P_{\ell+1}^{(i)}(\nu)-P_{\ell+2}^{(i)}(\nu)=1 \\
& \quad \geq m_{\ell+1}^{(i-1)}(\nu)-2 m_{\ell+1}^{(i)}(\nu)+m_{\ell+1}^{(i+1)}(\nu)=m_{\ell+1}^{(i-1)}(\nu)+m_{\ell+1}^{(i+1)}(\nu),
\end{aligned}
$$

where we have used the fact that $m_{\ell+1}^{(i)}(\nu)=0$ which follows from the assumption $j=\ell$ and $\ell+2 \leq \ell_{(i)}$. Since we know that $m_{k}^{(i+1)}(\nu)=0$ for all $\ell+1<k<\ell_{(i)}$, the relation $\ell=\ell^{(i)}<\ell_{(i+1)}<\ell_{(i)}$ with $m_{\ell_{(i+1)}}^{(i+1)}(\nu)>0$ forces that $\ell_{(i+1)}=\ell+1$ and $m_{\ell+1}^{(i+1)}(\nu)=1$. Now recall the relation $\ell<\ell^{(i+1)}$ of (5.16) which is shown independently to the present proposition. By the assumption $\ell_{(i+1)}<\ell_{(i)}$ and the relation $\ell^{(i+1)} \leq \ell_{(i+1)}$ that follows from the definition, we also have $\ell=\ell^{(i)}<\ell^{(i+1)}<\ell_{(i)}$ with $m_{\ell^{(i+1)}}^{(i+1)}(\nu)>0$. By definition of the operation $\delta$, the two strings $\left(\ell^{(i+1)}, P_{\ell^{(i+1)}}^{(i)}(\nu)\right)$ and $\left(\ell_{(i+1)}, P_{\ell_{(i+1)}^{(i)}}^{(\nu))}\right.$ of $(\nu, J)^{(i+1)}$ must be distinct. However, as we have seen in the previous paragraph, there is only one string $\left(k, x_{k}\right)$ of $(\nu, J)^{(i+1)}$ under the restriction $\ell<k<\ell_{(i)}$. This is a contradiction.

In conclusion, we obtain $P_{\ell_{(i)}-1}^{(i)}(\nu)>x_{\ell}+1$ in this Case (VII).

Proof of "Propositions $5.30 \& 5.31 \Longrightarrow \overline{\widetilde{\boldsymbol{\nu}}}=\widetilde{\widetilde{\boldsymbol{\nu}}}$ ". Step 1. Let us consider the case $\widetilde{f}_{i} \circ \delta$. The first $\delta$ creates the strings $\left(\ell-1, P_{\ell-1}^{(i)}(\bar{\nu})\right)$ and $\left(\ell_{(i)}-1, P_{\ell_{(i)}-1}^{(i)}(\bar{\nu})\right)$ of $(\bar{\nu}, \bar{J})^{(i)}$. Recall that the rigging of the latter string satisfies $P_{\ell_{(i)}-1}^{(i)}(\bar{\nu})>x_{\ell}$ by Proposition 5.31. Then, since the operation $\delta$ does not change the riggings of unchanged strings, the relation (5.15) and $m_{\ell}^{(i)}(\nu)=1$ imply that the string $\left(\ell-1, x_{\ell}\right)$ is the longest strings among the string with rigging $x_{\ell}$, hence $\tilde{f}_{i}$ will act on this string.

Step 2. Let us consider the case $\delta \circ \widetilde{f}_{i}$. Recall that by definition of $\tilde{f}_{i}$ we have $\widetilde{\ell}^{(a)}=\ell^{(a)}$ for all $a<i$. From Lemma 5.2(1) the relation (5.14) implies that the string $\left(\ell+1, x_{\ell}-1\right)$ of $(\widetilde{\nu}, \widetilde{J})^{(i)}$ created by $\widetilde{f}_{i}$ is singular. Since $m_{\ell}^{(i)}(\nu)=1$ we know that there is no singular string of $(\widetilde{\nu}, \widetilde{J})^{(i)}$ that is shorter than or equal to $\ell$ and longer than $\widetilde{\ell}^{(i-1)}$. Thus we have $\widetilde{\ell}^{(i)}=\ell+1$. Then, since $\widetilde{f}_{i}$ does not change coriggings of $(\widetilde{\nu}, \widetilde{J})^{(i+1)}$, the relation $(5.16)$ implies that $\widetilde{\ell}^{(a)}=\ell^{(a)}$ for all $i<a$ as well as $\tilde{\ell}_{(a)}=\ell_{(a)}$ for all $a$.

Step 3. To summarize, we have $\overline{\widetilde{\nu}}^{(a)}=\widetilde{\bar{\nu}}^{(a)}=\bar{\nu}^{(a)}$ for all $a \neq i$ and $\overline{\widetilde{\nu}}^{(i)}=\widetilde{\bar{\nu}}^{(i)}$ are obtained by removing a box from a length $\ell_{(i)}$ singular string of $\nu^{(i)}$. Hence we have $\overline{\widetilde{\nu}}=\widetilde{\bar{\nu}}$.

Proofs of $\overline{\widetilde{J}}=\widetilde{\bar{J}}$ will be given by case by case analysis assuming the relation $\overline{\widetilde{\nu}}=\widetilde{\bar{\nu}}$ along with the proof of Proposition 5.30.

\subsubsection{Proof of Proposition 5.30 for the case $\ell^{(i-1)}<\ell$}

We divide the proof into two cases depending on whether $\ell^{(i-1)}<\ell$ or $\ell^{(i-1)}=\ell$. In this subsection, we consider the case $\ell^{(i-1)}<\ell$.

To begin with, let us show the following property.

Proposition 5.32. Suppose that $\ell^{(i-1)}<\ell=\ell^{(i)}$ and $m_{\ell}^{(i)}(\nu)=1$. Then we have

$$
P_{\ell-1}^{(i)}(\nu)=x_{\ell}+1, \quad P_{\ell}^{(i)}(\nu)=x_{\ell}, \quad P_{\ell+1}^{(i)}(\nu)=x_{\ell}+1 .
$$


Moreover we have

$$
m_{\ell}^{(i-1)}(\nu)=m_{\ell}^{(i+1)}(\nu)=0 .
$$

For the proof, we prepare the following lemma.

Lemma 5.33. Suppose that $\ell^{(i-1)}<\ell=\ell^{(i)}$ and $m_{\ell}^{(i)}(\nu)=1$. Then we have the following relations:

$$
P_{\ell-1}^{(i)}(\nu)>P_{\ell}^{(i)}(\nu), \quad(2) \quad P_{\ell}^{(i)}(\nu)<P_{\ell+1}^{(i)}(\nu) .
$$

Proof. (1) Note that from the assumptions $m_{\ell}^{(i)}(\nu)=1$ and $\ell=\ell^{(i)}$, we see that the string $\left(\ell, x_{\ell}\right)$ is singular which implies that $x_{\ell}=P_{\ell}^{(i)}(\nu)$. Let $j$ be the largest integer such that $j \leq \ell-1$ and $m_{j}^{(i)}(\nu)>0$. If there is no such $j$, set $j=0$ and $x_{j}=0$.

We divide the proof into two cases depending on whether $j=\ell-1$ or $j<\ell-1$.

Step 1. Let us consider the case $j=\ell-1$. Then the string $\left(\ell-1, x_{\ell-1}\right)$ is non-singular since its length satisfies $\ell^{(i-1)} \leq \ell-1<\ell^{(i)}$. Thus we have $P_{\ell-1}^{(i)}(\nu)>x_{\ell-1}$. Recall that we have $x_{\ell-1} \geq x_{\ell}$ by the minimality of $x_{\ell}$. Then $x_{\ell}=P_{\ell}^{(i)}(\nu)$ implies that $P_{\ell-1}^{(i)}(\nu)>P_{\ell}^{(i)}(\nu)$ in this case.

Step 2. Let us consider the case $j<\ell-1$. Then we have $x_{j} \geq x_{\ell}$ by definition of $x_{\ell}$ and $\ell>0$. Thus we have $P_{j}^{(i)}(\nu) \geq x_{\ell}$ by $P_{j}^{(i)}(\nu) \geq x_{j}$. Since $x_{\ell}=P_{\ell}^{(i)}(\nu)$ we obtain $P_{j}^{(i)}(\nu) \geq P_{\ell}^{(i)}(\nu)$. Then by the convexity of $P_{k}^{(i)}(\nu)$ between $j \leq k \leq \ell$ we obtain $P_{\ell-1}^{(i)}(\nu) \geq \min \left\{P_{j}^{(i)}(\nu), P_{\ell}^{(i)}(\nu)\right\}=$ $P_{\ell}^{(i)}(\nu)$. Suppose if possible that $P_{\ell-1}^{(i)}(\nu)=P_{\ell}^{(i)}(\nu)$. Then by the convexity of the vacancy numbers the only possibility that is compatible with $P_{j}^{(i)}(\nu) \geq P_{\ell}^{(i)}(\nu)$ is the case $P_{j}^{(i)}(\nu)=$ $\cdots=P_{\ell}^{(i)}(\nu)$.

(i) Suppose that $\ell^{(i-1)} \leq j$. Recall that the string $\left(j, x_{j}\right)$ satisfies $x_{j} \leq P_{j}^{(i)}(\nu)=P_{\ell}^{(i)}(\nu)=x_{\ell}$. On the other hand, by the minimality of $x_{\ell}$, we have $x_{j} \geq x_{\ell}$. Thus $x_{j}=P_{j}^{(i)}(\nu)$. Then the length of the singular string $\left(j, x_{j}\right)$ satisfies $\ell^{(i-1)} \leq j<\ell^{(i)}$ which is in contradiction to the definition of $\ell^{(i)}$. Thus we conclude that $P_{\ell-1}^{(i)}(\nu)>P_{\ell}^{(i)}(\nu)$ if $\ell^{(i-1)} \leq j$.

(ii) Suppose that $j<\ell^{(i-1)}$. Then the relation of $P_{k}^{(i)}(\nu)$ between $\ell^{(i-1)}-1 \leq k \leq \ell^{(i-1)}+1$ must be strictly convex due to the existence of the length $\ell^{(i-1)}$ string at $\nu^{(i-1)}$. This is in contradiction to the relation $P_{j}^{(i)}(\nu)=\cdots=P_{\ell}^{(i)}(\nu)$.

Thus we conclude that $P_{\ell-1}^{(i)}(\nu)>P_{\ell}^{(i)}(\nu)$.

(2) Since $\widetilde{f}_{i}$ does not vanish, the rigging for the new string created by $\widetilde{f}_{i}$ does not exceed the corresponding vacancy number. Therefore, from the proof of Lemma 5.2(1), we have $P_{\ell+1}^{(i)}(\nu) \geq$ $x_{\ell}+1$. Since the string $\left(\ell, x_{\ell}\right)$ is singular we have $P_{\ell}^{(i)}(\nu)=x_{\ell}$. Combining both relations we obtain $P_{\ell}^{(i)}(\nu)<P_{\ell+1}^{(i)}(\nu)$.

Proof of Proposition 5.32. We apply Lemma 3.12 with $m_{\ell}^{(i)}(\nu)=1$. Then $P_{\ell-1}^{(i)}(\nu), P_{\ell}^{(i)}(\nu)$ and $P_{\ell+1}^{(i)}(\nu)-2$ have to satisfy the convex relation. The only possibility that is compatible with the relation $P_{\ell-1}^{(i)}(\nu)>P_{\ell}^{(i)}(\nu)<P_{\ell+1}^{(i)}(\nu)$ is $P_{\ell-1}^{(i)}(\nu)=P_{\ell}^{(i)}(\nu)+1=P_{\ell+1}^{(i)}(\nu)$.

From Lemma 3.13 we have

$$
\begin{aligned}
& -P_{\ell-1}^{(i)}(\nu)+2 P_{\ell}^{(i)}(\nu)-P_{\ell+1}^{(i)}(\nu)=-2 \\
& \quad \geq m_{\ell}^{(i-1)}(\nu)-2 m_{\ell}^{(i)}(\nu)+m_{\ell}^{(i+1)}(\nu)=m_{\ell}^{(i-1)}(\nu)-2+m_{\ell}^{(i+1)}(\nu),
\end{aligned}
$$


that is, $0 \geq m_{\ell}^{(i-1)}(\nu)+m_{\ell}^{(i+1)}(\nu)$. Since $m_{\ell}^{(i-1)}(\nu) \geq 0$ and $m_{\ell}^{(i+1)}(\nu) \geq 0$ we conclude that $m_{\ell}^{(i-1)}(\nu)=m_{\ell}^{(i+1)}(\nu)=0$.

Proof of Proposition 5.30 for the case $\ell^{(i-1)}<\ell$. By $(5.17)$ we have $P_{\ell+1}^{(i)}(\nu)=x_{\ell}+1$, that is, (5.14). By the assumption $\ell^{(i-1)}<\ell$ we see $\ell^{(i-1)} \leq \ell-1$. Thus we obtain $P_{\ell-1}^{(i)}(\bar{\nu})=$ $P_{\ell-1}^{(i)}(\nu)-1=x_{\ell}$, that is, (5.15). Finally, from (5.18) we have $m_{\ell}^{(i+1)}(\nu)=0$. Therefore we have $\ell<\ell^{(i+1)}$, that is, (5.16).

In view of the proof of $\overline{\widetilde{\nu}}=\widetilde{\bar{\nu}}$, we can show $\overline{\widetilde{J}}=\widetilde{\bar{J}}$ for this case as follows.

Proposition 5.34. Suppose that $\ell^{(i-1)}<\ell=\ell^{(i)}<\ell_{(i)}$ and $m_{\ell}^{(i)}(\nu)=1$. Then we have $\overline{\widetilde{J}}=\widetilde{\bar{J}}$.

Proof. From (5.18) it is enough to analyze the string $\left(\ell, x_{\ell}\right)$ of $(\nu, J)^{(i)}$ where $x_{\ell}=P_{\ell}^{(i)}(\nu)$. Then we have

$$
\begin{aligned}
& \left(\ell, x_{\ell}\right) \stackrel{\widetilde{f}_{i}}{\longmapsto}\left(\ell+1, x_{\ell}-1\right) \stackrel{\delta}{\longmapsto}\left(\ell, P_{\ell}^{(i)}(\overline{\widetilde{\nu}})\right), \\
& \left(\ell, x_{\ell}\right) \stackrel{\delta}{\longmapsto}\left(\ell-1, P_{\ell-1}^{(i)}(\bar{\nu})\right) \stackrel{\widetilde{f}_{i}}{\longmapsto}\left(\ell, P_{\ell-1}^{(i)}(\bar{\nu})-1\right) .
\end{aligned}
$$

Recall that we have $\ell^{(i-1)}<\ell=\ell^{(i)}<\ell^{(i+1)}$. Then we have $P_{\ell}^{(i)}(\overline{\widetilde{\nu}})=P_{\ell}^{(i)}(\nu)-1=x_{\ell}-1$ since we have $P_{\ell}^{(i)}(\nu)=x_{\ell}$ by (5.17). Similarly we have $P_{\ell-1}^{(i)}(\bar{\nu})-1=P_{\ell-1}^{(i)}(\nu)-2=x_{\ell}-1$ since we have $P_{\ell-1}^{(i)}(\nu)=x_{\ell}+1$ by $(5.17)$.

\subsubsection{Proof of Proposition 5.30 for the case $\ell^{(i-1)}=\ell$}

We begin by showing the following property.

Proposition 5.35. Suppose that $\ell^{(i-1)}=\ell=\ell^{(i)}$ and $m_{\ell}^{(i)}(\nu)=1$. Then we have

$$
P_{\ell-1}^{(i)}(\nu)=P_{\ell}^{(i)}(\nu)=x_{\ell}, \quad P_{\ell+1}^{(i)}(\nu)=x_{\ell}+1 .
$$

Moreover, we have

$$
m_{\ell}^{(i-1)}(\nu)=1, \quad m_{\ell}^{(i+1)}(\nu)=0 .
$$

For the proof, we prepare the following lemma.

Lemma 5.36. Suppose that $\ell^{(i-1)}=\ell=\ell^{(i)}$ and $m_{\ell}^{(i)}(\nu)=1$. Then we have the following relations:

$$
\text { (1) } \quad P_{\ell-1}^{(i)}(\nu) \geq P_{\ell}^{(i)}(\nu), \quad(2) \quad P_{\ell}^{(i)}(\nu)<P_{\ell+1}^{(i)}(\nu) .
$$

Proof. (1) Let $j$ be the largest integer such that $j \leq \ell-1$ and $m_{j}^{(i)}(\nu)>0$. If there is no such $j$, set $j=0$. We divide the proof into two cases depending on whether $j=\ell-1$ or $j<\ell-1$.

Step 1. Let us consider the case $j=\ell-1$. Since the string $\left(\ell, x_{\ell}\right)$ is singular, we have $P_{\ell}^{(i)}(\nu)=x_{\ell}$, and by the minimality of $x_{\ell}$ we have $x_{\ell} \leq x_{\ell-1}$. Since $x_{\ell-1} \leq P_{\ell-1}^{(i)}$, we conclude that $P_{\ell-1}^{(i)}(\nu) \geq P_{\ell}^{(i)}(\nu)$.

Step 2. Let us consider the case $j<\ell-1$. Recall that the assumptions $\ell=\ell^{(i)}$ and $m_{\ell}^{(i)}(\nu)=1$ imply that the string $\left(\ell, x_{\ell}\right)$ is singular and thus $P_{\ell}^{(i)}(\nu)=x_{\ell}$. If $j>0$, then by the minimality of $\ell$ with $j<\ell$ we have $P_{j}^{(i)}(\nu) \geq x_{j} \geq x_{\ell}=P_{\ell}^{(i)}(\nu)$. If $j=0$ we have $P_{0}^{(i)}(\nu)=0$. In this case, we also have $P_{j}^{(i)}(\nu) \geq P_{\ell}^{(i)}(\nu)$ since we have $P_{\ell}^{(i)}(\nu)=x_{\ell} \leq 0$ which is 
the consequence of $\ell>0$. In both cases, by the convexity of $P_{k}^{(i)}(\nu)$ between $j \leq k \leq \ell$ we have $P_{\ell-1}^{(i)}(\nu) \geq \min \left\{P_{j}^{(i)}(\nu), P_{\ell}^{(i)}(\nu)\right\}=P_{\ell}^{(i)}(\nu)$.

Proof of (2) is the same with the previous case.

Proof of Proposition 5.35. Since $\ell^{(i-1)}=\ell$ we have $m_{\ell}^{(i-1)}(\nu)>0$. Then Lemma 3.12 with $m_{\ell}^{(i)}(\nu)=1$ claims that $P_{\ell-1}^{(i)}(\nu), P_{\ell}^{(i)}(\nu)$ and $P_{\ell+1}^{(i)}(\nu)-2$ are strictly convex. The only possibility that is compatible with the relation $P_{\ell-1}^{(i)}(\nu) \geq P_{\ell}^{(i)}(\nu)<P_{\ell+1}^{(i)}(\nu)$ is $P_{\ell-1}^{(i)}(\nu)+1=P_{\ell}^{(i)}(\nu)+1=$ $P_{\ell+1}^{(i)}(\nu)$. Then from Lemma 3.13 we have

$$
\begin{aligned}
& -P_{\ell-1}^{(i)}(\nu)+2 P_{\ell}^{(i)}(\nu)-P_{\ell+1}^{(i)}(\nu)=-1 \\
& \quad \geq m_{\ell}^{(i-1)}(\nu)-2 m_{\ell}^{(i)}(\nu)+m_{\ell}^{(i+1)}(\nu)=m_{\ell}^{(i-1)}(\nu)-2+m_{\ell}^{(i+1)}(\nu),
\end{aligned}
$$

that is, $1 \geq m_{\ell}^{(i-1)}(\nu)+m_{\ell}^{(i+1)}(\nu)$. Since we know that $m_{\ell}^{(i-1)}(\nu)>0$ and $m_{\ell}^{(i+1)}(\nu) \geq 0$, the only possibility is $m_{\ell}^{(i-1)}(\nu)=1$ and $m_{\ell}^{(i+1)}(\nu)=0$.

Proof of Proposition 5.30 for the case $\ell^{(i-1)}=\ell$. By $(5.19)$ we have $P_{\ell+1}^{(i)}(\nu)=x_{\ell}+1$, that is, (5.14). By the assumption we have $\ell-1<\ell^{(i-1)}$. Then from (5.19) we have $P_{\ell-1}^{(i)}(\bar{\nu})=P_{\ell-1}^{(i)}(\nu)=x_{\ell}$, which implies (5.15). Finally $m_{\ell}^{(i+1)}(\nu)=0$ implies that $\ell<\ell^{(i+1)}$, that is, (5.16).

This completes the whole proof of Proposition 5.30.

Again, using the proof of $\overline{\widetilde{\nu}}=\widetilde{\bar{\nu}}$, we can show $\overline{\widetilde{J}}=\widetilde{\bar{J}}$ for this case as follows.

Proposition 5.37. Suppose that $\ell^{(i-1)}=\ell=\ell^{(i)}<\ell_{(i)}$ and $m_{\ell}^{(i)}(\nu)=1$. Then we have $\overline{\widetilde{J}}=\widetilde{\bar{J}}$.

Proof. By (5.20) it is enough to analyze the string $\left(\ell, x_{\ell}\right)$ of $(\nu, J)^{(i)}$. Then we have

$$
\begin{array}{llll}
\left(\ell, x_{\ell}\right) & \stackrel{\widetilde{f}_{i}}{\longmapsto}\left(\ell+1, x_{\ell}-1\right) & \stackrel{\delta}{\longmapsto} & \left(\ell, P_{\ell}^{(i)}(\overline{\widetilde{\nu}})\right), \\
\left(\ell, x_{\ell}\right) & \stackrel{\delta}{\longmapsto}\left(\ell-1, P_{\ell-1}^{(i)}(\bar{\nu})\right) \stackrel{\widetilde{f}_{i}}{\longmapsto}\left(\ell, P_{\ell-1}^{(i)}(\bar{\nu})-1\right) .
\end{array}
$$

From $\ell^{(i-1)}=\ell<\ell^{(i+1)}$ by Proposition 5.30, $P_{\ell}^{(i)}(\nu)=x_{\ell}$ by (5.19) and the fact that the length of the string $\left(\ell, x_{\ell}\right)$ is preserved under both $\delta \circ \widetilde{f}_{i}$ and $\widetilde{f}_{i} \circ \delta$, we have $P_{\ell}^{(i)}(\overline{\widetilde{\nu}})=P_{\ell}^{(i)}(\nu)-1=x_{\ell}-1$. On the other hand, we have $P_{\ell-1}^{(i)}(\bar{\nu})-1=P_{\ell-1}^{(i)}(\nu)-1=x_{\ell}-1$ since we have $\ell-1<\ell^{(i-1)}$ and $P_{\ell-1}^{(i)}(\nu)=x_{\ell}$ by $(5.19)$.

Example 5.38. Here we give an example for Proposition 5.35. Consider the following rigged configuration $(\nu, J)$ of type $\left(B^{1,1}\right)^{\otimes 3} \otimes B^{1,3} \otimes B^{2,1} \otimes B^{2,2} \otimes B^{3,1}$ of $D_{5}^{(1)}$

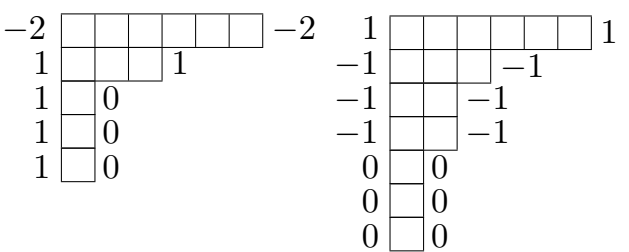
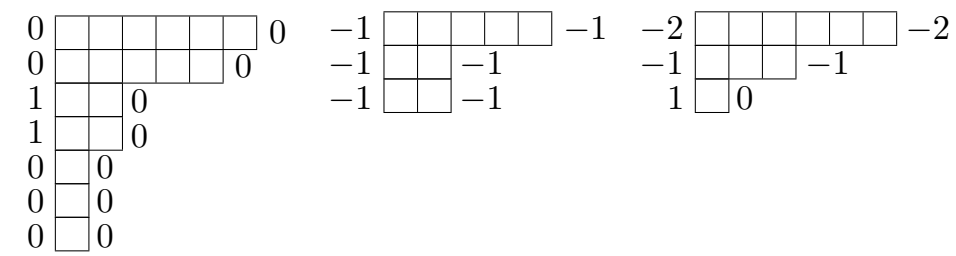

The corresponding tensor product $\Phi^{-1}(\nu, J)$ is

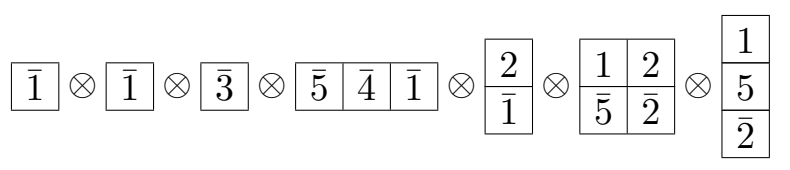

Then $(\bar{\nu}, \bar{J})$ is 

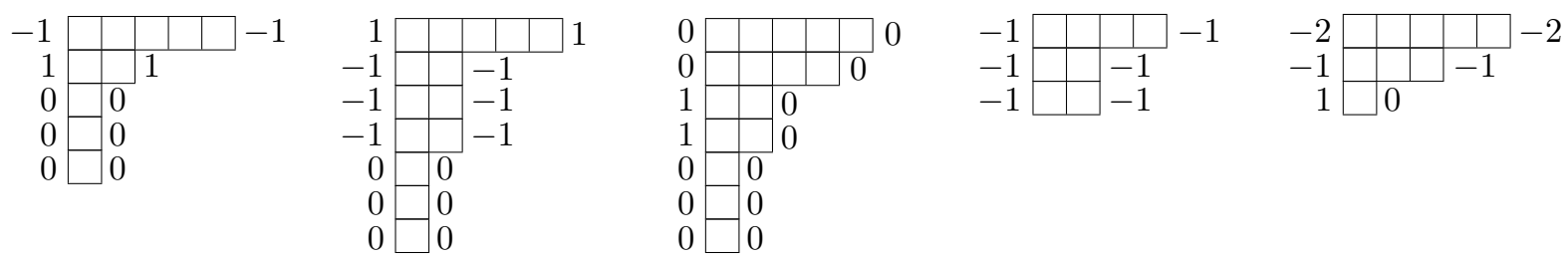

and $(\widetilde{\nu}, \widetilde{J})$ is
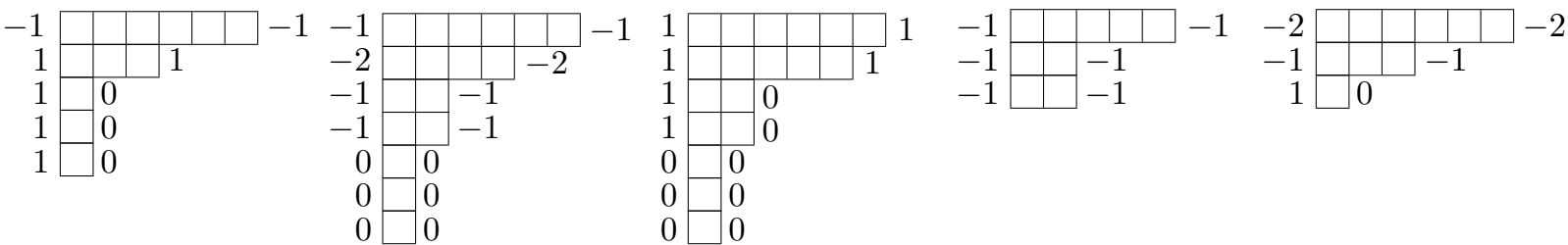

In this example, we have $\ell^{(1)}=\ell=\ell^{(2)}=3, m_{3}^{(2)}(\nu)=1$ and $x_{\ell}=-1$. As proved in Proposition 5.35, we have $P_{2}^{(2)}(\nu)=P_{3}^{(2)}(\nu)=-1=x_{\ell}, P_{4}^{(2)}(\nu)=0=x_{\ell}+1, m_{3}^{(1)}(\nu)=1$ and $m_{3}^{(3)}(\nu)=0$. Finally $(\overline{\widetilde{\nu}}, \overline{\widetilde{J}})=(\widetilde{\bar{\nu}}, \widetilde{\bar{J}})$ is

\begin{tabular}{l|l|l|l|}
0 & & & \\
1 & & & \\
0 & &
\end{tabular}

\begin{tabular}{|c|c|c|}
\hline-1 & & \\
\hline & & -2 \\
\hline-1 & & -1 \\
\hline-1 & & -1 \\
\hline 0 & 0 & \\
\hline $\begin{array}{l}0 \\
0\end{array}$ & $\begin{array}{l}0 \\
0\end{array}$ & \\
\hline
\end{tabular}
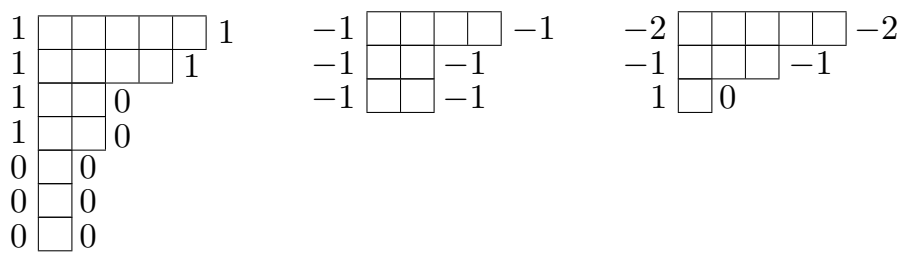

\subsection{Proof for Case D (4)}

\subsubsection{Outline}

We will prove the following proposition in the latter part of this subsection.

Proposition 5.39. Suppose that $\ell=\ell^{(i)}=\ell_{(i)}$ and $m_{\ell}^{(i)}(\nu)=2$. Then the following relations hold:

$$
\begin{aligned}
& P_{\ell+1}^{(i)}(\nu)=x_{\ell}+1, \\
& P_{\ell-1}^{(i)}(\bar{\nu})=x_{\ell}, \\
& \ell+1 \leq \ell_{(i-1)} .
\end{aligned}
$$

Proof of "Proposition 5.39 $\Longrightarrow \overline{\widetilde{\boldsymbol{\nu}}}=\widetilde{\widetilde{\boldsymbol{\nu}}}$ ". Step 1. Let us consider the case $\widetilde{f}_{i} \circ \delta$. The first $\delta$ creates the two strings $\left(\ell-1, P_{\ell-1}^{(i)}(\bar{\nu})\right)$ of $(\bar{\nu}, \bar{J})^{(i)}$. Since the operation $\delta$ does not change the riggings of unchanged strings, the relation (5.22) and $m_{\ell}^{(i)}(\nu)=2$ imply that the strings $\left(\ell-1, x_{\ell}\right)$ are the longest strings among the strings with rigging $x_{\ell}$, hence $\tilde{f}_{i}$ will act on one of these strings.

Step 2. Let us consider the case $\delta \circ \tilde{f}_{i}$. Recall that by definition of $\tilde{f}_{i}$ we have $\tilde{\ell}^{(a)}=\ell^{(a)}$ for all $a<i$. From Lemma 5.2(1) the relation (5.21) implies that the string $\left(\ell+1, x_{\ell}-1\right)$ of $(\widetilde{\nu}, \widetilde{J})^{(i)}$ created by $\widetilde{f}_{i}$ is singular. Then from the assumption $m_{\ell}^{(i)}(\nu)=2$ we see that there is one singular string of length $\ell$ and at least one singular string of length $\ell+1$ within $(\widetilde{\nu}, \widetilde{J})^{(i)}$. Thus we have $\widetilde{\ell}^{(a)}=\ell^{(a)}$ for all $i \leq a, \widetilde{\ell}_{(a)}=\ell_{(a)}$ for all $i<a$ and $\widetilde{\ell}_{(i)}=\ell+1$. Then from (5.23) we have $\tilde{\ell}_{(i-1)}=\ell_{(i-1)}$ and hence $\tilde{\ell}_{(a)}=\ell_{(a)}$ for all $a<i$.

Step 3. To summarize, we have $\overline{\widetilde{\nu}}^{(a)}=\widetilde{\bar{\nu}}^{(a)}=\bar{\nu}^{(a)}$ for all $a \neq i$ and $\overline{\widetilde{\nu}}^{(i)}=\widetilde{\bar{\nu}}^{(i)}$ are obtained by removing a box from a length $\ell$ row of $\nu^{(i)}$. Hence we have $\overline{\widetilde{\nu}}=\widetilde{\bar{\nu}}$. 
Proofs of $\overline{\widetilde{J}}=\widetilde{\bar{J}}$ will be given by case by case analysis along with the proof of Proposition 5.39 assuming the relation $\overline{\widetilde{\nu}}=\widetilde{\bar{\nu}}$ we have just proved.

\subsubsection{Proof of Proposition 5.39 for the case $\ell^{(i-1)}<\ell$}

We divide the proof of Proposition 5.39 into two cases according to whether $\ell^{(i-1)}<\ell$ or $\ell^{(i-1)}=\ell$. In this subsection, we consider the case $\ell^{(i-1)}<\ell$. Throughout the proof of Proposition 5.39, let $j$ be the largest integer such that $j \leq \ell-1$ and $m_{j}^{(i)}(\nu)>0$. If there is no such $j$, set $j=0$.

Proposition 5.40. Suppose that $\ell=\ell^{(i)}=\ell_{(i)}, m_{\ell}^{(i)}(\nu)=2$ and $\ell^{(i-1)}<\ell$. Then we have

$$
P_{\ell-1}^{(i)}(\nu)=x_{\ell}+1, \quad P_{\ell}^{(i)}(\nu)=x_{\ell}, \quad P_{\ell+1}^{(i)}(\nu)=x_{\ell}+1 .
$$

Moreover, we have

$$
m_{\ell}^{(i-1)}(\nu)=0, \quad m_{\ell}^{(i+1)}(\nu)=2 .
$$

For the proof of this proposition, we prepare the following lemma.

Lemma 5.41. Suppose that $\ell=\ell^{(i)}=\ell_{(i)}, m_{\ell}^{(i)}(\nu)=2$ and $\ell^{(i-1)}<\ell$. Then we have

$$
\text { (1) } P_{\ell-1}^{(i)}(\nu)>P_{\ell}^{(i)}(\nu) \text {, }
$$$$
\text { (2) } \quad P_{\ell}^{(i)}(\nu)<P_{\ell+1}^{(i)}(\nu) \text {. }
$$

Proof. Recall that by definition of $\delta$, the strings $\left(\ell^{(i)}, P_{\ell^{(i)}}^{(i)}(\nu)\right)$ and $\left(\ell_{(i)}, P_{\ell_{(i)}}^{(i)}(\nu)\right)$ of $(\nu, J)^{(i)}$ must be different. Then the assumptions $\ell=\ell^{(i)}=\ell_{(i)}$ and $m_{\ell}^{(i)}(\nu)=2$ imply that the string $\left(\ell, x_{\ell}\right)$ must be singular. In particular, we have $P_{\ell}^{(i)}(\nu)=x_{\ell}$. Then we can use the same arguments of Lemma 5.33 to prove the assertions.

Proof of Proposition 5.40. According to the previous lemma, we can write $P_{\ell-1}^{(i)}(\nu)$ and $P_{\ell+1}^{(i)}(\nu)$ as follows:

$$
P_{\ell-1}^{(i)}(\nu)=x_{\ell}+\varepsilon_{1}, \quad P_{\ell+1}^{(i)}(\nu)=x_{\ell}+\varepsilon_{2},
$$

where $\varepsilon_{1}, \varepsilon_{2} \geq 1$. Then Lemma 3.13 with $l=\ell$ reads

$$
\begin{aligned}
& -P_{\ell-1}^{(i)}(\nu)+2 P_{\ell}^{(i)}(\nu)-P_{\ell+1}^{(i)}(\nu)=-\varepsilon_{1}-\varepsilon_{2} \\
& \quad \geq m_{\ell}^{(i-1)}(\nu)-2 m_{\ell}^{(i)}(\nu)+m_{\ell}^{(i+1)}(\nu)=m_{\ell}^{(i-1)}(\nu)-4+m_{\ell}^{(i+1)}(\nu),
\end{aligned}
$$

that is,

$$
4-\varepsilon_{1}-\varepsilon_{2} \geq m_{\ell}^{(i-1)}(\nu)+m_{\ell}^{(i+1)}(\nu) .
$$

Note that the assumption $\ell^{(i)}=\ell_{(i)}$ implies that $\ell^{(i+1)}=\ell_{(i+1)}=\ell$ by definition of $\delta$. In particular, we have $m_{\ell}^{(i+1)}(\nu) \geq 2$. Then the only possibility that is compatible with the above inequality is $\varepsilon_{1}=\varepsilon_{2}=1, m_{\ell}^{(i-1)}(\nu)=0$ and $m_{\ell}^{(i+1)}(\nu)=2$.

Proof of Proposition $\mathbf{5 . 3 9}$ for the case $\boldsymbol{\ell}^{(\boldsymbol{i}-\mathbf{1})}<\boldsymbol{\ell}$. The relation (5.21) is proved in (5.24). As for (5.22), since $\ell^{(i-1)}<\ell=\ell^{(i)}$ we have $P_{\ell-1}^{(i)}(\bar{\nu})=P_{\ell-1}^{(i)}(\nu)-1=x_{\ell}$ by (5.24). Finally, by (5.25) we have $m_{\ell}^{(i-1)}(\nu)=0$. Recall that we have $\ell_{(i-1)} \geq \ell_{(i)}$ by definition of $\delta$. Then, since we are assuming that $\ell_{(i)}=\ell$, we have $\ell_{(i-1)}>\ell$, that is, (5.23). 
Assuming that $\overline{\widetilde{\nu}}=\widetilde{\bar{\nu}}$, we can show $\overline{\widetilde{J}}=\widetilde{\bar{J}}$ for this case as follows.

Proposition 5.42. Suppose that $\ell^{(i-1)}<\ell=\ell^{(i)}=\ell_{(i)}$ and $m_{\ell}^{(i)}(\nu)=2$. Then we have $\overline{\widetilde{J}}=\widetilde{\bar{J}}$.

Proof. From the proof of $\overline{\widetilde{\nu}}=\widetilde{\bar{\nu}}$ after Proposition 5.39, we see that it is enough to look at the string $\left(\ell, x_{\ell}\right)$ of $(\nu, J)^{(i)}$ which is changed by both $\widetilde{f}_{i}$ and $\delta$ in both $\widetilde{f}_{i} \circ \delta$ and $\delta \circ \widetilde{f}_{i}$. Then we have

$$
\begin{array}{llll}
\left(\ell, x_{\ell}\right) & \stackrel{\tilde{f}_{i}}{\longmapsto}\left(\ell+1, x_{\ell}-1\right) & \stackrel{\delta}{\longmapsto} & \left(\ell, P_{\ell}^{(i)}(\overline{\widetilde{\nu}})\right), \\
\left(\ell, x_{\ell}\right) \stackrel{\delta}{\longmapsto}\left(\ell-1, P_{\ell-1}^{(i)}(\bar{\nu})\right) & \stackrel{\widetilde{f}_{i}}{\longmapsto}\left(\ell, P_{\ell-1}^{(i)}(\bar{\nu})-1\right) .
\end{array}
$$

Recall that we have

$$
\widetilde{\ell}^{(i-1)}<\widetilde{\ell}^{(i)}=\widetilde{\ell}^{(i+1)}=\widetilde{\ell}_{(i+1)}=\ell<\widetilde{\ell}_{(i)}=\ell+1 \leq \widetilde{\ell}_{(i-1)}
$$

by the assumption $\ell^{(i-1)}<\ell=\ell^{(i)}=\ell_{(i)}$ and the arguments given in the proof of $\overline{\widetilde{\nu}}=\widetilde{\bar{\nu}}$. Then we have $P_{\ell}^{(i)}(\overline{\widetilde{\nu}})=P_{\ell}^{(i)}(\nu)-1=x_{\ell}-1$ since we have $P_{\ell}^{(i)}(\nu)=x_{\ell}$ by (5.24). Similarly we have $P_{\ell-1}^{(i)}(\bar{\nu})-1=P_{\ell-1}^{(i)}(\nu)-2=x_{\ell}-1$ since we have $P_{\ell-1}^{(i)}(\nu)=x_{\ell}+1$ by $(5.24)$.

\subsubsection{Proof of Proposition 5.39 for the case $\ell^{(i-1)}=\ell$}

Proposition 5.43. Suppose that $\ell=\ell^{(i)}=\ell_{(i)}, m_{\ell}^{(i)}(\nu)=2$ and $\ell^{(i-1)}=\ell$. Then we have

$$
P_{\ell-1}^{(i)}(\nu)=P_{\ell}^{(i)}(\nu)=x_{\ell}, \quad P_{\ell+1}^{(i)}(\nu)=x_{\ell}+1 .
$$

Moreover, we have

$$
m_{\ell}^{(i-1)}(\nu)=1, \quad m_{\ell}^{(i+1)}(\nu)=2 .
$$

For the proof of this proposition, we prepare the following lemma.

Lemma 5.44. Suppose that $\ell=\ell^{(i)}=\ell_{(i)}, m_{\ell}^{(i)}(\nu)=2$ and $\ell^{(i-1)}=\ell$. Then we have

$$
P_{\ell-1}^{(i)}(\nu) \geq P_{\ell}^{(i)}(\nu), \quad(2) \quad P_{\ell}^{(i)}(\nu)<P_{\ell+1}^{(i)}(\nu) .
$$

Proof. Since we are assuming that $\ell^{(i)}=\ell_{(i)}$ and $m_{\ell}^{(i)}(\nu)=2$, we see that the strings $\left(\ell, x_{\ell}\right)$ of $(\nu, J)^{(i)}$ are singular. Then we can use the same arguments of the proof of Lemma 5.36.

Proof of Proposition 5.43. According to the previous lemma, we can express $P_{\ell-1}^{(i)}(\nu)$ and $P_{\ell+1}^{(i)}(\nu)$ as follows:

$$
P_{\ell-1}^{(i)}(\nu)=x_{\ell}+\varepsilon_{1}, \quad P_{\ell+1}^{(i)}(\nu)=x_{\ell}+\varepsilon_{2},
$$

where $\varepsilon_{1} \geq 0$ and $\varepsilon_{2} \geq 1$. Then Lemma 3.13 with $l=\ell$ reads

$$
\begin{aligned}
& -P_{\ell-1}^{(i)}(\nu)+2 P_{\ell}^{(i)}(\nu)-P_{\ell+1}^{(i)}(\nu)=-\varepsilon_{1}-\varepsilon_{2} \\
& \quad \geq m_{\ell}^{(i-1)}(\nu)-2 m_{\ell}^{(i)}(\nu)+m_{\ell}^{(i+1)}(\nu)=m_{\ell}^{(i-1)}(\nu)-4+m_{\ell}^{(i+1)}(\nu),
\end{aligned}
$$

that is,

$$
4-\varepsilon_{1}-\varepsilon_{2} \geq m_{\ell}^{(i-1)}(\nu)+m_{\ell}^{(i+1)}(\nu) .
$$

Since we are assuming that $\ell^{(i-1)}=\ell$, we have $m_{\ell}^{(i-1)}(\nu) \geq 1$. Also, from the assumption $\ell^{(i)}=\ell_{(i)}$ we see that $\ell^{(i+1)}=\ell_{(i+1)}$ by definition of $\delta$. Thus we have $m_{\ell}^{(i+1)}(\nu) \geq 2$. Then the only possibility that is compatible with the above inequality is that $\varepsilon_{1}=0, \varepsilon_{2}=1, m_{\ell}^{(i-1)}(\nu)=1$ and $m_{\ell}^{(i+1)}(\nu)=2$. 
Proof of Proposition 5.39 for the case $\boldsymbol{\ell}^{(\boldsymbol{i}-\mathbf{1})}=\boldsymbol{\ell}$. The relation (5.21) is proved in (5.26). As for (5.22), since $\ell^{(i-1)}=\ell^{(i)}=\ell$ we have $P_{\ell-1}^{(i)}(\bar{\nu})=P_{\ell-1}^{(i)}(\nu)=x_{\ell}$ by (5.26). Finally, by $(5.27)$ we have $m_{\ell}^{(i-1)}(\nu)=1$. Since we are assuming that $\ell^{(i-1)}=\ell$, this string corresponds to $\left(\ell^{(i-1)}, x_{\ell^{(i-1)}}\right)$ which is different from the string $\left(\ell_{(i-1)}, x_{\ell_{(i-1)}}\right)$. Since we have $\ell^{(i-1)} \leq \ell_{(i-1)}$ by the definition of $\delta$, we must have $\ell<\ell_{(i-1)}$, that is, (5.23).

Assuming that $\overline{\widetilde{\nu}}=\widetilde{\bar{\nu}}$, we can show $\overline{\widetilde{J}}=\widetilde{\bar{J}}$ for this case as follows.

Proposition 5.45. Suppose that $\ell=\ell^{(i)}=\ell_{(i)}, m_{\ell}^{(i)}(\nu)=2$ and $\ell^{(i-1)}=\ell$. Then we have $\overline{\widetilde{J}}=\widetilde{\bar{J}}$.

Proof. Recall that we can assume that $\tilde{f}_{i}$ acts on the same string $\left(\ell, x_{\ell}\right)$ of $(\nu, J)^{(i)}$ in both $\widetilde{f}_{i} \circ \delta$ and $\delta \circ \widetilde{f}_{i}$. Then we have

$$
\begin{aligned}
& \left(\ell, x_{\ell}\right) \stackrel{\widetilde{f}_{i}}{\longmapsto}\left(\ell+1, x_{\ell}-1\right) \stackrel{\delta}{\longmapsto}\left(\ell, P_{\ell}^{(i)}(\overline{\widetilde{\nu}})\right), \\
& \left(\ell, x_{\ell}\right) \stackrel{\delta}{\longmapsto}\left(\ell-1, P_{\ell-1}^{(i)}(\bar{\nu})\right) \stackrel{\widetilde{f}_{i}}{\longmapsto}\left(\ell, P_{\ell-1}^{(i)}(\bar{\nu})-1\right) .
\end{aligned}
$$

Recall that we have

$$
\widetilde{\ell}^{(i-1)}=\widetilde{\ell}^{(i)}=\widetilde{\ell}^{(i+1)}=\tilde{\ell}_{(i+1)}=\ell<\tilde{\ell}_{(i)}=\ell+1 \leq \tilde{\ell}_{(i-1)}
$$

by the assumption $\ell^{(i-1)}=\ell^{(i)}=\ell_{(i)}=\ell$ and the arguments given in the proof of $\overline{\widetilde{\nu}}=\widetilde{\bar{\nu}}$. Then we have $P_{\ell}^{(i)}(\overline{\widetilde{\nu}})=P_{\ell}^{(i)}(\nu)-1=x_{\ell}-1$ since we have $P_{\ell}^{(i)}(\nu)=x_{\ell}$ by (5.26). Similarly we have $P_{\ell-1}^{(i)}(\bar{\nu})-1=P_{\ell-1}^{(i)}(\nu)-1=x_{\ell}-1$ since we have $P_{\ell-1}^{(i)}(\nu)=x_{\ell}$ by (5.26).

Example 5.46. Consider the following rigged configuration $(\nu, J)$ of type $\left(B^{1,1}\right)^{\otimes 3} \otimes B^{1,3} \otimes$ $B^{2,1} \otimes B^{2,2} \otimes B^{3,1}$ of $D_{5}^{(1)}$

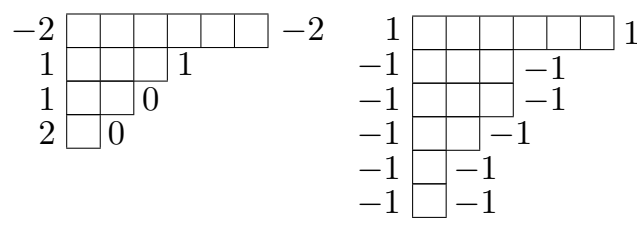
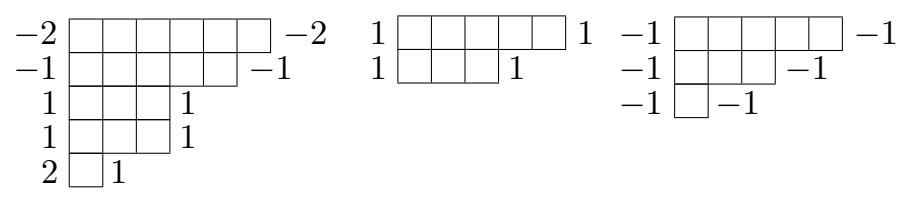

The corresponding tensor product $\Phi^{-1}(\nu, J)$ is

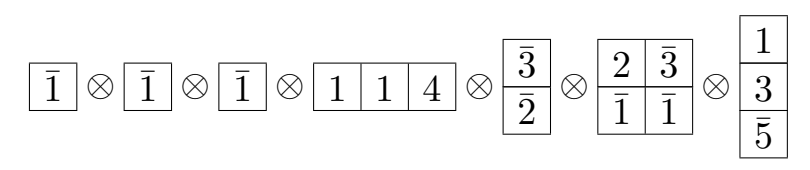

\begin{tabular}{|c|c|}
\hline 1 & \begin{tabular}{l|l} 
& \\
\end{tabular} \\
\hline-1 & -1 \\
\hline-1 & -1 \\
\hline-1 & -1 \\
\hline $\begin{array}{l}-1 \\
-1\end{array}$ & \\
\hline
\end{tabular}

Then $(\bar{\nu}, \bar{J})$ is

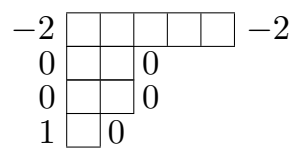
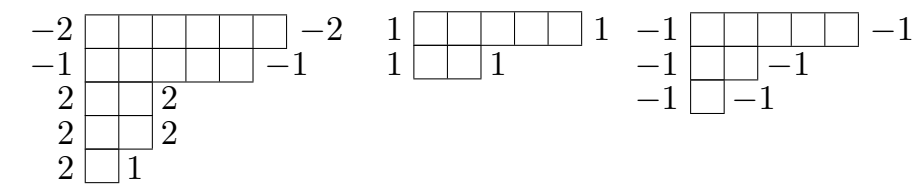

and $(\widetilde{\nu}, \widetilde{J})$ is
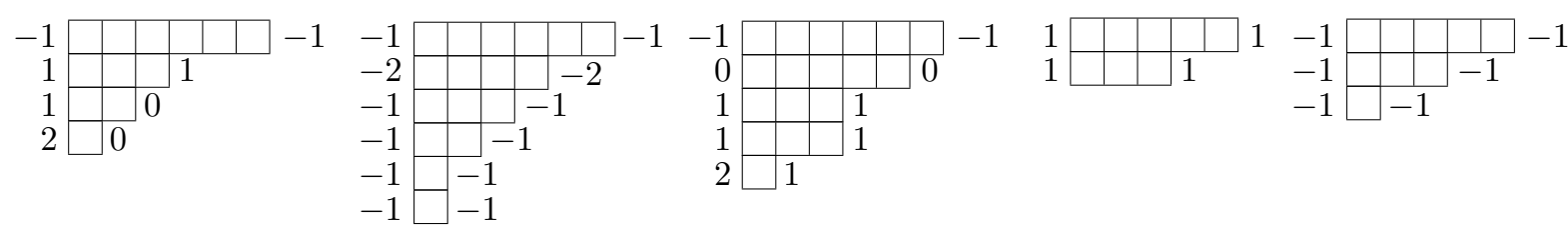
In this example, we have $\ell=\ell^{(1)}=\ell^{(2)}=\ell_{(2)}=3$ and $m_{3}^{(2)}(\nu)=2$. Then we have $x_{\ell}=-1$, $P_{2}^{(2)}(\nu)=P_{3}^{(2)}(\nu)=-1, P_{4}^{(2)}(\nu)=0, m_{3}^{(1)}(\nu)=1$ and $m_{3}^{(3)}(\nu)=2$ (see Proposition 5.43). We also have $P_{2}^{(2)}(\bar{\nu})=-1$ and $\ell_{(1)}=6 \geq \ell+1=4$ (see Proposition 5.39). Finally $(\overline{\widetilde{\nu}}, \overline{\widetilde{J}})=(\widetilde{\bar{\nu}}, \widetilde{\bar{J}})$ is

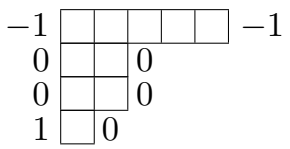

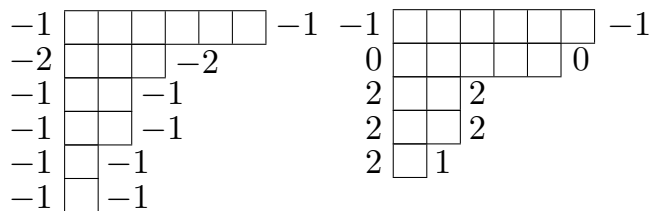

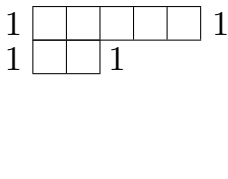

\begin{tabular}{|c|c|}
\hline-1 & \\
\hline-1 & -1 \\
\hline & -1 \\
\hline
\end{tabular}

\subsection{Proof for Case E: preliminary steps}

\subsubsection{Classification}

Recall that the defining condition for Case $\mathrm{E}$ is $\ell^{(i)}<\ell$. We further classify this case as follows.

(1) $\ell^{(i)}<\ell$ and $\ell+1<\ell_{(i+1)}$,

(2) $\ell^{(i)}<\ell$ and $\ell_{(i+1)} \leq \ell+1<\ell_{(i)}=\infty$,

(3) $\ell^{(i)}<\ell$ and $\ell_{(i+1)} \leq \ell+1 \leq \ell_{(i)}<\infty$,

(4) $\ell^{(i)}<\ell$ and $\ell_{(i)}=\ell$,

(5) $\ell^{(i)}<\ell$ and $\ell_{(i)}<\ell$

\subsubsection{A common property for Case $\mathrm{E}$}

The fundamental observation for this case is the following property.

Proposition 5.47. If $\ell^{(i)}<\ell$, we have

$$
P_{\ell^{(i)}-1}^{(i)}(\bar{\nu}) \geq x_{\ell} .
$$

Proof. We follow the classification of Lemma 5.1.

Case (I). Let us consider the case $\ell^{(i-1)}=\ell^{(i)}$. Then by Lemma 5.1, we have to show $P_{\ell^{(i)}-1}^{(i)}(\nu) \geq x_{\ell}$. Suppose if possible that $P_{\ell^{(i)}-1}^{(i)}(\nu)<x_{\ell}$. If $\ell^{(i)}=1$, then we have $0=$ $P_{\ell^{(i)}-1}^{(i)}(\nu)<x_{\ell}$ which is a contradiction since we have $x_{\ell} \leq 0$ by $0<\ell$. Therefore we assume $\ell^{(i)} \geq 2$ in the sequel. From the assumption $\ell^{(i)}<\ell$ we have $P_{\ell^{(i)}}^{(i)}(\nu) \geq x_{\ell^{(i)}} \geq x_{\ell}$. Let $j$ be the length of the longest string of $\nu^{(i)}$ that are strictly shorter than $\ell^{(i)}$. If $j=\ell^{(i)}-1$, then we have $x_{j} \leq P_{j}^{(i)}(\nu)<x_{\ell}$, which contradicts the minimality of $x_{\ell}$. Thus we assume that $j<\ell^{(i)}-1$. We apply the convexity relation of $P_{k}^{(i)}$ for $j \leq k \leq \ell^{(i)}$ with the relation $P_{\ell^{(i)}-1}^{(i)}(\nu)<P_{\ell^{(i)}}^{(i)}(\nu)$ and obtain $x_{j} \leq P_{j}^{(i)}(\nu)<P_{\ell^{(i)}-1}^{(i)}(\nu)<x_{\ell}$, which is a contradiction. Therefore we see that $\nu^{(i)}$ does not contain strings that are strictly shorter than $\ell^{(i)}$. Again we can use the convexity relation of $P_{k}^{(i)}$ for $0 \leq k \leq \ell^{(i)}$ with the relation $P_{\ell^{(i)}-1}^{(i)}(\nu)<P_{\ell^{(i)}}^{(i)}(\nu)$ and obtain the relation $0=P_{0}^{(i)}(\nu)<P_{\ell(i)-1}^{(i)}(\nu)<x_{\ell}$. This is a contradiction since we have $x_{\ell} \leq 0$. Thus we have proved $P_{\ell^{(i)}-1}^{(i)}(\bar{\nu}) \geq x_{\ell}$ for the case $\ell^{(i-1)}=\ell^{(i)}$.

Case (II). Let us consider the case $\ell^{(i-1)}<\ell^{(i)}$. Then by Lemma 5.1, we have to show $P_{\ell^{(i)}-1}^{(i)}(\nu)>x_{\ell}$. Suppose if possible that $P_{\ell^{(i)}-1}^{(i)}(\nu) \leq x_{\ell}$. Let $j$ be the length of the longest row of $\nu^{(i)}$ that are strictly shorter than $\ell^{(i)}$. Suppose that $j=\ell^{(i)}-1$. Then we have $x_{j} \leq$ $P_{j}^{(i)}(\nu) \leq x_{\ell}$. By the minimality of $x_{\ell}$ this relation implies that $x_{j}=P_{j}^{(i)}(\nu)$, that is, the string 
$\left(j, x_{j}\right)$ is singular in $(\nu, J)^{(i)}$. This is in contradiction to the definition of $\ell^{(i)}$ since we have $\ell^{(i-1)} \leq j=\ell^{(i)}-1<\ell^{(i)}$.

Therefore we assume $j<\ell^{(i)}-1$ in the sequel. If $P_{\ell^{(i)}-1}^{(i)}(\nu)<x_{\ell}$, from $P_{\ell^{(i)}}^{(i)}(\nu) \geq x_{\ell^{(i)}} \geq x_{\ell}$ we have $P_{\ell^{(i)}-1}^{(i)}(\nu)<P_{\ell^{(i)}}^{(i)}(\nu)$ and by the convexity relation we have $x_{j} \leq P_{j}^{(i)}(\nu)<P_{\ell^{(i)}-1}^{(i)}(\nu)$, that is $x_{j}<x_{\ell}$. This is a contradiction. Therefore we must have $P_{\ell^{(i)}-1}^{(i)}(\nu)=x_{\ell}$. If $P_{\ell^{(i)}}^{(i)}(\nu)>x_{\ell}$, by the similar argument we obtain $x_{j}<x_{\ell}$, which is a contradiction. Thus we conclude that $P_{\ell^{(i)}}^{(i)}(\nu)=x_{\ell}$. Suppose that $\ell^{(i-1)} \leq j$. Then from the convexity relation of $P_{k}^{(i)}(\nu)$ for $j \leq k \leq$ $\ell^{(i)}$, we obtain $P_{j}^{(i)}(\nu) \leq P_{\ell^{(i)}-1}^{(i)}(\nu)=P_{\ell^{(i)}}^{(i)}(\nu)=x_{\ell}$ and from the requirement $P_{j}^{(i)}(\nu) \geq x_{j} \geq x_{\ell}$ we obtain $P_{j}^{(i)}(\nu)=x_{j}$, that is, the string $\left(j, x_{j}\right)$ is singular. But this is a contradiction since $\ell^{(i-1)} \leq j<\ell^{(i)}$. Thus we have $j<\ell^{(i-1)}$. Since $m_{\ell^{(i-1)}}^{(i-1)}(\nu)>0$ the convexity relation for $P_{k}^{(i)}(\nu)$ between $\ell^{(i-1)}-1 \leq k \leq \ell^{(i-1)}+1$ must be strict. Thus we obtain $x_{\ell} \geq P_{\ell^{(i-1)}}^{(i)}(\nu)>P_{j}^{(i)}(\nu) \geq x_{j}$, which is a contradiction.

Therefore we conclude that $(\nu, J)^{(i)}$ does not contain strings that are strictly shorter than $\ell^{(i)}$. Then from the convexity relation of $P_{k}^{(i)}(\nu)$ between $0 \leq k \leq \ell^{(i)}$, we obtain $x_{\ell} \geq P_{\ell^{(i)}-1}^{(i)}(\nu) \geq$ $P_{\ell^{(i-1)}}^{(i)}(\nu)>P_{0}^{(i)}(\nu)=0$, which is in contradiction to the requirement $x_{\ell} \leq 0$. Thus we have proved $P_{\ell^{(i)}-1}^{(i)}(\bar{\nu}) \geq x_{\ell}$ for the case $\ell^{(i-1)}<\ell^{(i)}$.

\subsection{Proof for Case E (1)}

To begin with we prepare the following property.

Proposition 5.48. Suppose that $\ell^{(i)}<\ell$ and $\ell+1<\ell_{(i+1)}$. Then we have

$$
P_{\ell_{(i)}-1}^{(i)}(\bar{\nu})>x_{\ell}
$$

Proof. We follow the classification of Lemma 5.1. From the assumption, we see that $\ell^{(i)}<$ $\ell_{(i+1)}$. Then we only need to consider cases $(\mathrm{V}),(\mathrm{VI})$ and (VII). During the proof of the proposition, let $j$ be the largest integer such that $j<\ell_{(i)}$ and $m_{j}^{(i)}(\nu)>0$. Since $\ell^{(i)}<\ell<$ $\ell_{(i+1)} \leq \ell_{(i)}$ we have $\ell \leq j$. Proofs for cases (V) and (VII) are the same as the corresponding parts of the proof of Proposition 5.3.

Case (VI). In this case, we have to show $P_{\ell_{(i)}-1}^{(i)}(\nu)>x_{\ell}$. Suppose if possible that $P_{\ell_{(i)}-1}^{(i)}(\nu) \leq x_{\ell}$. First, let us consider the case $j=\ell_{(i)}-1$. Then the corresponding string satisfies $x_{\ell_{(i)}-1} \leq P_{\ell_{(i)}-1}^{(i)}(\nu) \leq x_{\ell}$. Now recall that we have $\ell<\ell_{(i)}-1$ since we have $\ell+1<\ell_{(i+1)} \leq \ell_{(i)}$ by the assumption. Thus the minimality of $x_{\ell}$ requires that $x_{\ell}<x_{\ell_{(i)}-1}$. This is a contradiction. Similarly, if $\ell<j<\ell_{(i)}-1$, we have $x_{\ell} \geq P_{\ell_{(i)}-1}^{(i)}(\nu) \geq \min \left\{P_{\ell_{(i)}}^{(i)}(\nu), P_{j}^{(i)}(\nu)\right\}$ which implies that $x_{\ell} \geq x_{\ell_{(i)}}$ or $x_{\ell} \geq x_{j}$. This is in contradiction to the requirements $x_{\ell}<x_{\ell_{(i)}}$ and $x_{\ell}<x_{j}$ derived from $\ell<\ell_{(i)}$ and $\ell<j$, respectively.

Finally let us consider the case $\ell=j$. Recall that we have $P_{\ell}^{(i)}(\nu) \geq x_{\ell}, P_{\ell_{(i)}-1}^{(i)}(\nu) \leq x_{\ell}$ and $P_{\ell_{(i)}}^{(i)}(\nu)>x_{\ell}$. However these relations are in contradiction to the convexity relation of $P_{k}^{(i)}(\nu)$ between $\ell \leq k \leq \ell_{(i)}$ which we can use by $\ell<\ell_{(i)}-1$.

Proposition 5.49. Suppose that $\ell^{(i)}<\ell$ and $\ell+1<\ell_{(i+1)}$. Then we have the following identities:
(1) $\quad \overline{\widetilde{\nu}}=\widetilde{\bar{\nu}}$,
(2) $\overline{\widetilde{J}}=\widetilde{\bar{J}}$. 
Proof. Step 1. Let us consider the case $\delta \circ \widetilde{f}_{i}$. Since $\tilde{f}_{i}$ does not change coriggings of the untouched strings, and since $\tilde{f}_{i}$ creates the string whose length satisfies $\ell^{(i)}<\ell+1<\ell_{(i+1)}$, we have $\widetilde{\ell}^{(a)}=\ell^{(a)}$ and $\tilde{\ell}_{(a)}=\ell_{(a)}$ for all $a$.

Step 2. Let us consider the case $\widetilde{f}_{i} \circ \delta$. Since we are assuming that $\ell^{(i)}<\ell<\ell_{(i)}$ the string $\left(\ell, x_{\ell}\right)$ remains as it is after $\delta$. Recall that we have $P_{\ell^{(i)}-1}^{(i)}(\bar{\nu}) \geq x_{\ell}$ by Proposition 5.47 and $P_{\ell_{(i)}-1}^{(i)}(\bar{\nu})>x_{\ell}$ by the previous proposition. Thus the string $\left(\ell, x_{\ell}\right)$ has the largest length among the strings of $(\widetilde{\nu}, \widetilde{J})^{(i)}$ that have the smallest rigging.

Step 3. To summarize, we have $\overline{\widetilde{\nu}}^{(a)}=\widetilde{\bar{\nu}}^{(a)}=\bar{\nu}^{(a)}$ for all $a \neq i$ and $\overline{\widetilde{\nu}}^{(i)}=\widetilde{\bar{\nu}}^{(i)}$ is obtained by adding a box to the $\ell$-th column of $\bar{\nu}^{(i)}$. The statement $\widetilde{\widetilde{J}}=\widetilde{\bar{J}}$ is obtained by the equality $P_{k}^{(i)}(\overline{\widetilde{\nu}})=P_{k}^{(i)}(\widetilde{\bar{\nu}})$ for arbitrary $k$ which is the consequence of $\overline{\widetilde{\nu}}=\widetilde{\bar{\nu}}$.

\subsection{Proof for Case E (2)}

Let us consider the case $\ell^{(i)}<\ell$ and $\ell_{(i+1)} \leq \ell+1<\ell_{(i)}=\infty$. We divide the proof into two cases according to whether $\widetilde{f}_{i}$ creates a singular string or not.

\subsubsection{Case 1: $\widetilde{f}_{i}$ creates a non-singular string}

Proposition 5.50. Suppose that we have $\ell^{(i)}<\ell$ and $\ell_{(i+1)} \leq \ell+1<\ell_{(i)}=\infty$. Suppose further that $\widetilde{f}_{i}$ acting on $(\nu, J)$ creates a non-singular string. Then we have the following identities:
(1) $\overline{\widetilde{\nu}}=\widetilde{\bar{\nu}}$,
(2) $\quad \overline{\widetilde{J}}=\widetilde{\bar{J}}$.

Proof. Step 1. Let us consider the case $\delta \circ \widetilde{f}_{i}$. Since $\widetilde{f}_{i}$ does not change coriggings of the untouched strings, and since $\widetilde{f}_{i}$ creates a non-singular string, we have $\widetilde{\ell}^{(a)}=\ell^{(a)}$ and $\widetilde{\ell}_{(a)}=\ell_{(a)}$ for all $a$.

Step 2. Let us consider the case $\tilde{f}_{i} \circ \delta$. Since we are assuming that $\ell^{(i)}<\ell$ and $\ell_{(i)}=\infty$ the string $\left(\ell, x_{\ell}\right)$ remains as it is after $\delta$. Recall that we have $P_{\ell^{(i)}-1}^{(i)}(\bar{\nu}) \geq x_{\ell}$ by Proposition 5.47. Thus the string $\left(\ell, x_{\ell}\right)$ has the largest length among the strings of $(\widetilde{\nu}, \widetilde{J})^{(i)}$ that have the smallest rigging.

Step 3. To summarize, we have $\overline{\widetilde{\nu}}^{(a)}=\widetilde{\bar{\nu}}^{(a)}=\bar{\nu}^{(a)}$ for all $a \neq i$ and $\overline{\widetilde{\nu}}^{(a)}=\widetilde{\bar{\nu}}^{(a)}$ is obtained by adding a box to the $\ell$-th column of $\bar{\nu}^{(i)}$. The statement $\overline{\widetilde{J}}=\widetilde{\bar{J}}$ follows from $\overline{\widetilde{\nu}}=\widetilde{\bar{\nu}}$.

\subsubsection{Case 2: $\tilde{f}_{i}$ creates a singular string}

Proposition 5.51. Suppose that we have $\ell^{(i)}<\ell$ and $\ell_{(i+1)} \leq \ell+1<\ell_{(i)}=\infty$. Suppose further that $\widetilde{f}_{i}$ acting on $(\nu, J)$ creates a singular string. Then we have

$$
\tilde{f}_{i} \circ \delta(\nu, J)=0 .
$$

Proof. Since $\ell_{(i)}=\infty, \delta$ changes the string $\left(\ell^{(i)}, P_{\ell^{(i)}}^{(i)}(\nu)\right)$ of $(\nu, J)^{(i)}$ only. In particular, from $\ell^{(i)}<\ell$ the string $\left(\ell, x_{\ell}\right)$ remains as it is after the application of $\delta$. Since we know that $P_{\ell^{(i)}-1}^{(i)}(\bar{\nu}) \geq x_{\ell}$ by Proposition 5.47, the string $\left(\ell, x_{\ell}\right)$ is the largest string among the strings with the smallest rigging within $(\bar{\nu}, \bar{J})^{(i)}$. Thus the next $\tilde{f}_{i}$ acts on this string and produces $\left(\ell+1, x_{\ell}-1\right)$.

Let us compute $P_{\ell+1}^{(i)}(\widetilde{\bar{\nu}})$. Since $\widetilde{f}_{i}$ acting on $(\nu, J)$ creates a singular string, we have $P_{\ell+1}^{(i)}(\nu)=$ $x_{\ell}+1$ by Lemma 5.2. Then we have $P_{\ell+1}^{(i)}(\bar{\nu})=P_{\ell+1}^{(i)}(\nu)-1=x_{\ell}$ since we have $\ell^{(i-1)} \leq \ell^{(i)} \leq$ 
$\ell^{(i+1)} \leq \ell+1$ and $\ell_{(i+1)} \leq \ell+1<\ell_{(i)}=\infty$ by the assumptions. Finally, since $\tilde{f}_{i}$ adds a box to the $(\ell+1)$-th column of $(\bar{\nu}, \bar{J})^{(i)}$, we have $P_{\ell+1}^{(i)}(\widetilde{\bar{\nu}})=P_{\ell+1}^{(i)}(\bar{\nu})-2=x_{\ell}-2$. Then we see that the string $\left(\ell+1, x_{\ell}-1\right)$ of $(\widetilde{\bar{\nu}}, \widetilde{\bar{J}})^{(i)}$ satisfies $x_{\ell}-1>P_{\ell+1}^{(i)}(\widetilde{\bar{\nu}})$. Therefore we conclude that $\tilde{f}_{i} \circ \delta(\nu, J)=0$.

Example 5.52. Consider the following rigged configuration $(\nu, J)$ of type $\left(B^{1,1}\right)^{\otimes 4} \otimes B^{1,3} \otimes$ $B^{2,1} \otimes B^{2,2} \otimes B^{3,1}$ of $D_{5}^{(1)}$

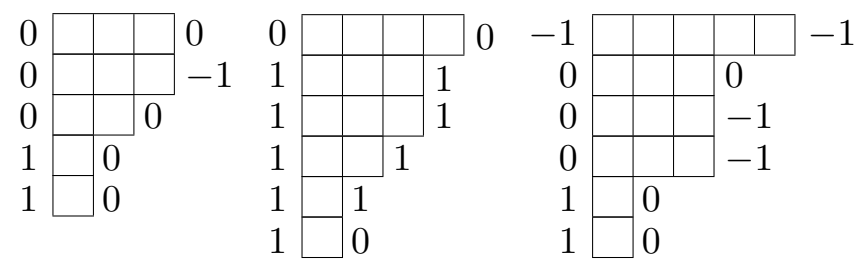

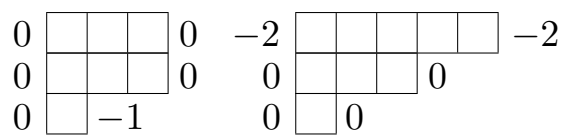

$\widetilde{f}_{2}$ acts on the string $\left(\ell, x_{\ell}\right)=(4,0)$ of $(\nu, J)^{(2)}$ and makes it into $(5,-1)$ of $(\widetilde{\nu}, \widetilde{J})^{(2)}$. Since we have $P_{5}^{(2)}(\widetilde{\nu})=-1$, the latter string is singular. In particular, we see that $\widetilde{f}_{2}(\nu, J) \neq 0$. The corresponding tensor product $\Phi^{-1}(\nu, J)$ is

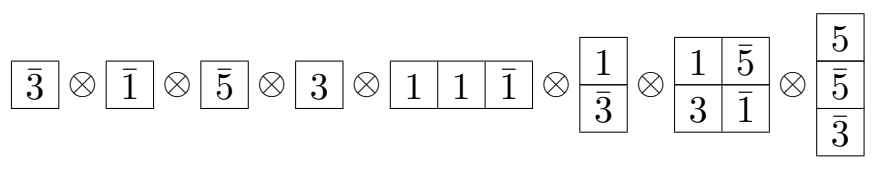

Then $(\bar{\nu}, \bar{J})$ is

\begin{tabular}{|c|c|c|c|c|c|c|c|c|}
\hline 0 & & & & & 0 & -1 & & -1 \\
\hline 0 & & -1 & 1 & & 1 & -1 & & -1 \\
\hline 0 & 0 & & 1 & & 1 & -1 & & -1 \\
\hline 0 & 0 & & 1 & 1 & & 0 & & 0 \\
\hline 0 & 0 & & 1 & 1 & & 1 & 0 & \\
\hline & & & & 0 & & 1 & 0 & \\
\hline
\end{tabular}

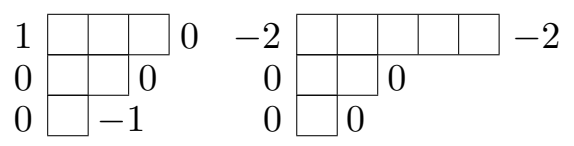

Thus we have $\ell^{(2)}=2<\ell=4$ and $\ell_{(3)}=5=\ell+1<\ell_{(2)}=\infty$ (see Proposition 5.51). Indeed, we have $P_{5}^{(2)}(\widetilde{\bar{\nu}})=-2$ and thus $\widetilde{f}_{2}(\bar{\nu}, \bar{J})=0$.

\subsection{Proof for Case E (3)}

Let us consider the case $\ell^{(i)}<\ell$ and $\ell_{(i+1)} \leq \ell+1 \leq \ell_{(i)}<\infty$. As in Case C, we use the following classification:

(1) $m_{\ell+1}^{(i)}(\nu)>0$ and $\widetilde{f}_{i}$ creates a singular string, or $\ell+1=\ell_{(i)}$ and $\widetilde{f}_{i}$ creates a non-singular string,

(2) $m_{\ell+1}^{(i)}(\nu)=0$ and $\tilde{f}_{i}$ creates a singular string,

(3) $\ell+1<\ell_{(i)}$ and $\widetilde{f}_{i}$ creates a non-singular string.

\subsubsection{Proof for Case 1}

Proposition 5.53. Suppose that $\ell^{(i)}<\ell, \ell_{(i+1)} \leq \ell+1 \leq \ell_{(i)}<\infty, m_{\ell+1}^{(i)}(\nu)>0$ and $\tilde{f}_{i}$ creates a singular string. Then we have the following identities:
(1) $\quad \overline{\widetilde{\nu}}=\widetilde{\bar{\nu}}$,
(2) $\quad \overline{\widetilde{J}}=\widetilde{\bar{J}}$. 
Proof. Since we have $P_{\ell^{(i)}-1}^{(i)}(\bar{\nu}) \geq x_{\ell}$ by Proposition 5.47, we can use the same arguments of the proof of Proposition 5.9 if we replace $\ell^{(i)}\left(\right.$ resp. $\left.\ell^{(i-1)}\right)$ there by $\ell_{(i)}\left(\right.$ resp. $\left.\ell_{(i+1)}\right)$ and neglect the arguments concerning the string $\ell_{(i)}$ there.

Proposition 5.54. Suppose that $\ell^{(i)}<\ell, \ell_{(i+1)} \leq \ell+1 \leq \ell_{(i)}<\infty, \ell+1=\ell_{(i)}$ and $\tilde{f}_{i}$ creates a non-singular string. Then we have the following identities:
(1) $\overline{\widetilde{\nu}}=\widetilde{\bar{\nu}}$,
(2) $\quad \overline{\widetilde{J}}=\widetilde{\bar{J}}$.

Proof. As in Case C we can reduce the proof to the previous proposition.

\subsubsection{Proof for Case 2}

As in Case C, we show the following property:

Proposition 5.55. Suppose that $\ell^{(i)}<\ell, \ell_{(i+1)} \leq \ell+1<\ell_{(i)}<\infty$ and $m_{\ell+1}^{(i)}(\nu)=0$. If $\widetilde{f}_{i}$ acting on $(\nu, J)$ creates a singular string, then the following two conditions are satisfied:

$$
P_{\ell_{(i)}-1}^{(i)}(\bar{\nu})=x_{\ell}, \quad m_{k}^{(i-1)}(\nu)=0 \text { for } \ell<k<\ell_{(i)} .
$$

Proof. As in Case C, the proof depend on the following two cases (see Lemma 5.13);

$$
\begin{aligned}
& \left(\ell, x_{\ell}\right) \text { is singular and } \ell=\ell_{(i+1)}-1 \text {, } \\
& \left(\ell, x_{\ell}\right) \text { is non-singular. }
\end{aligned}
$$

For the case (5.28) (resp. (5.29)) we can use the same arguments of Section 5.6.3 (resp. Section 5.6.4) by replacing $\ell^{(i)}\left(\right.$ resp. $\left.\ell^{(i-1)}\right)$ there by $\ell_{(i)}\left(\right.$ resp. $\left.\ell_{(i+1)}\right)$ to show the assertions.

Proposition 5.56. Suppose that $\ell^{(i)}<\ell, \ell_{(i+1)} \leq \ell+1<\ell_{(i)}<\infty$ and $m_{\ell+1}^{(i)}(\nu)=0$. Then we have $\overline{\widetilde{\nu}}=\widetilde{\bar{\nu}}$ and $\overline{\widetilde{J}}=\widetilde{\bar{J}}$.

Proof. Since we have $P_{\ell^{(i)}-1}^{(i)}(\bar{\nu}) \geq x_{\ell}$ by Proposition 5.47, we can use the same arguments of the proof of Proposition 5.12 if we replace $\ell^{(i)}\left(\right.$ resp. $\left.\ell^{(i-1)}\right)$ there by $\ell_{(i)}\left(\right.$ resp. $\left.\ell_{(i+1)}\right)$, neglect the arguments concerning the string $\ell_{(i)}$ there and use Proposition 5.55 instead of Proposition 5.11. Thus we obtain $\overline{\widetilde{\nu}}=\widetilde{\bar{\nu}}$. We can also use the same analysis given for Case $\mathrm{C}$ to show $\overline{\widetilde{J}}=\widetilde{\bar{J}}$.

\subsubsection{Proof for Case 3}

We can use the same arguments in Section 5.7 if we replace $\ell^{(i)}$ (resp. $\left.\ell^{(i-1)}\right)$ there by $\ell_{(i)}$ $\left(\right.$ resp. $\left.\ell_{(i+1)}\right)$ and neglect the arguments concerning the string $\ell_{(i)}$ there.

\subsection{Proof for Case E (4)}

\subsubsection{Preliminary}

In this case, we assume that $\ell^{(i)}<\ell$ and $\ell_{(i)}=\ell$. In view of the classification of Case $\mathrm{D}$ we have to consider the following two cases:

1) $\ell^{(i)}<\ell, \ell_{(i)}=\ell$ and $m_{\ell}^{(i)}(\nu)>1$,

3) $\ell^{(i)}<\ell, \ell_{(i)}=\ell$ and $m_{\ell}^{(i)}(\nu)=1$.

During the proof of Case E (4) we follow the above classification. In both cases, we have the following common property. 
Proposition 5.57. Assume that $\ell^{(i)}<\ell$ and $\ell_{(i)}=\ell$. Then the following relation holds:

$$
P_{\ell-1}^{(i)}(\bar{\nu}) \geq x_{\ell}
$$

Proof. We can use the same arguments in the proof of Proposition 5.23 if we replace $\ell^{(i)}$ $\left(\right.$ resp. $\left.\ell^{(i-1)}\right)$ there by $\ell_{(i)}\left(\right.$ resp. $\left.\ell_{(i+1)}\right)$. Here in order to evaluate the vacancy numbers in Case (a) and (b), we note that if we have $\ell-1<\ell_{(i+1)}$ we have $P_{\ell-1}^{(i)}(\bar{\nu}) \geq P_{\ell-1}^{(i)}(\nu)$ since we have $\ell^{(i)} \leq \ell-1$ by the assumption.

\subsubsection{Proof for Case 1}

Proposition 5.58. Assume that $\ell^{(i)}<\ell, \ell_{(i)}=\ell$ and $m_{\ell}^{(i)}(\nu)>1$. Then we have the following identities:

$$
\text { (1) } \quad \overline{\widetilde{\nu}}=\widetilde{\bar{\nu}}, \quad(2) \quad \overline{\widetilde{J}}=\widetilde{\bar{J}} .
$$

Proof. (1) Let us analyze the action of $\tilde{f}_{i}$ before and after the application of $\delta$. By the assumption $m_{\ell}^{(i)}(\nu)>1$ we can choose two distinct length $\ell$ strings $\left(\ell, x_{\ell}\right)$ and $\left(\ell, P_{\ell}^{(i)}(\nu)\right)$ of $(\nu, J)^{(i)}$ where $\widetilde{f}_{i}$ will act on the former one and $\delta$ will act on the latter one. Recall that we have $P_{\ell^{(i)}-1}^{(i)}(\bar{\nu}) \geq x_{\ell}$ by Proposition 5.47 and $P_{\ell_{(i)}-1}^{(i)}(\bar{\nu}) \geq x_{\ell}$ by Proposition 5.57. Thus both strings $\left(\ell^{(i)}-1, P_{\ell^{(i)}-1}^{(i)}(\bar{\nu})\right)$ and $\left(\ell_{(i)}-1, P_{\ell_{(i)}-1}^{(i)}(\bar{\nu})\right)$ of $(\bar{\nu}, \bar{J})^{(i)}$ are shorter than $\left(\ell, x_{\ell}\right)$ and their riggings are larger than or equal to $x_{\ell}$. Therefore $\tilde{f}_{i}$ will act on the same string $\left(\ell, x_{\ell}\right)$ in both $(\nu, J)$ and $(\bar{\nu}, \bar{J})$.

Let us analyze $\delta$. Recall that $\tilde{f}_{i}$ will not change the coriggings of the strings $\left(\ell^{(i)}, P_{\ell^{(i)}}^{(i)}(\nu)\right)$ and $\left(\ell_{(i)}, P_{\ell_{(i)}}^{(i)}(\nu)\right)$ of $(\nu, J)^{(i)}$. Since $\ell^{(i)}<\ell$ we have $\widetilde{\ell}^{(a)}=\ell^{(a)}$ for all $a$. Also, since $\ell_{(i)}<\ell+1$ we have $\tilde{\ell}_{(a)}=\ell_{(a)}$ for all $a$. To summarize $\delta$ chooses the same strings before and after the application of $\widetilde{f}_{i}$. Hence we obtain $\overline{\widetilde{\nu}}=\widetilde{\bar{\nu}}$.

(2) Since each $\widetilde{f}_{i}$ and $\delta$ chooses the same strings in $\delta \circ \widetilde{f}_{i}$ and $\widetilde{f}_{i} \circ \delta$, the coincidence $\overline{\widetilde{J}}=\widetilde{\bar{J}}$ is a consequence of $P_{k}^{(i)}(\overline{\widetilde{\nu}})=P_{k}^{(i)}(\widetilde{\bar{\nu}})$.

\subsubsection{Proof for Case 3}

Proposition 5.59. Assume that $\ell^{(i)}<\ell, \ell_{(i)}=\ell$ and $m_{\ell}^{(i)}(\nu)=1$. Then we have the following identities:

$$
\begin{aligned}
& P_{\ell+1}^{(i)}(\nu)=x_{\ell}+1, \\
& P_{\ell-1}^{(i)}(\bar{\nu})=x_{\ell}, \\
& \ell+1 \leq \ell_{(i-1)} .
\end{aligned}
$$

Proof. The proof is divided into two cases $\ell_{(i+1)}<\ell$ and $\ell_{(i+1)}=\ell$ and we can use the same arguments in the proof of Proposition 5.30 if we replace $\ell^{(i-1)}, \ell^{(i)}$ and $\ell^{(i+1)}$ there by $\ell_{(i+1)}, \ell_{(i)}$ and $\ell_{(i-1)}$, respectively.

Proposition 5.60. Assume that $\ell^{(i)}<\ell, \ell_{(i)}=\ell$ and $m_{\ell}^{(i)}(\nu)=1$. Then we have the following identities:
(1) $\quad \overline{\widetilde{\nu}}=\widetilde{\bar{\nu}}$,
(2) $\quad \overline{\widetilde{J}}=\widetilde{\bar{J}}$. 
Proof. Recall that we have $P_{\ell^{(i)}-1}^{(i)}(\bar{\nu}) \geq x_{\ell}$ by Proposition 5.47. Since we have $P_{\ell_{(i)}-1}^{(i)}(\bar{\nu})=x_{\ell}$ by (5.31), the string $\left(\ell^{(i)}-1, P_{\ell^{(i)}-1}^{(i)}(\bar{\nu})\right)$ of $(\bar{\nu}, \bar{J})^{(i)}$ will not be chosen by the next $\tilde{f}_{i}$. Then we can use the same arguments in the corresponding parts of Case D (3) if we replace $\ell^{(i)}$ $\left(\right.$ resp. $\left.\ell^{(i-1)}\right)$ there by $\ell_{(i)}\left(\right.$ resp. $\left.\ell_{(i+1)}\right)$ and neglect all the arguments related to $\ell_{(a)}$.

\subsection{Proof for Case E (5)}

In this case, we can show the following property.

Proposition 5.61. Suppose that $\ell_{(i)}<\ell$. Then we have

$$
P_{\ell_{(i)}-1}^{(i)}(\bar{\nu}) \geq x_{\ell}
$$

Proof. We can use the arguments of the proof of Proposition 5.47 if we replace $\ell^{(i)}\left(\right.$ resp. $\left.\ell^{(i-1)}\right)$ there by $\ell_{(i)}\left(\right.$ resp. $\left.\ell_{(i+1)}\right)$.

As the consequence of this proposition, we have the following result.

Proposition 5.62. Suppose that $\ell^{(i)}<\ell$ and $\ell_{(i)}<\ell$. Then we have $\overline{\widetilde{\nu}}=\widetilde{\bar{\nu}}$ and $\overline{\widetilde{J}}=\widetilde{\bar{J}}$.

Proof. Since we have $P_{\ell^{(i)}-1}^{(i)}(\bar{\nu}) \geq x_{\ell}$ by Proposition 5.47 and $P_{\ell_{(i)}-1}^{(i)}(\bar{\nu}) \geq x_{\ell}$ by Proposition 5.61, we see that $\tilde{f}_{i}$ acts on the same string before and after $\delta$. Also, since $\ell^{(i)} \leq \ell_{(i)}<\ell$ and the fact that $\tilde{f}_{i}$ does not change coriggings of untouched strings, we see that $\delta$ acts on the same strings before and after $\widetilde{f}_{i}$. Thus we have $\overline{\widetilde{\nu}}=\widetilde{\bar{\nu}}$. $\overline{\widetilde{J}}=\widetilde{\bar{J}}$ follows from the fact $P_{k}^{(i)}(\overline{\widetilde{\nu}})=P_{k}^{(i)}(\widetilde{\bar{\nu}})$ which is the consequence of $\overline{\widetilde{\nu}}=\widetilde{\widetilde{\nu}}$.

\section{Proof of Proposition 4.8: $\widetilde{f}_{i}$ version}

\subsection{Proof for (1)}

Proposition 6.1. Let us consider the rigged configuration $(\nu, J)$ of type $B^{1,1} \otimes \bar{B}$. Suppose that we have the commutativity of $\widetilde{f}_{i}$ and $\Phi$ for $\bar{B}$. Suppose that we have $\widetilde{f}_{i}(\nu, J) \neq 0$ and $\widetilde{f}_{i}(\bar{\nu}, \bar{J})=0$. Let $b=\Phi(\nu, J)$ and $b^{\prime}=\Phi(\bar{\nu}, \bar{J})$. Then we have one of the following two cases:

(1) $b=i \otimes b^{\prime}, \widetilde{f}_{i}(b)$ is defined, $\widetilde{f}_{i}\left(b^{\prime}\right)$ is undefined and $\Phi\left(\widetilde{f}_{i}(\nu, J)\right)=(i+1) \otimes b^{\prime}$,

(2) $b=\overline{i+1} \otimes b^{\prime}, \widetilde{f}_{i}(b)$ is defined, $\widetilde{f}_{i}\left(b^{\prime}\right)$ is undefined and $\Phi\left(\widetilde{f}_{i}(\nu, J)\right)=\bar{i} \otimes b^{\prime}$.

For the proof, we start by preparing the following properties.

Proposition 6.2. $\widetilde{f}_{i}(\nu, J) \neq 0$ and $\widetilde{f}_{i}(\bar{\nu}, \bar{J})=0$ if and only if one the following conditions is satisfied:

(1) $\ell^{(i-1)} \leq \ell+1<\ell^{(i)}=\infty$ and $\tilde{f}_{i}$ acting on $(\nu, J)$ creates a singular string,

(2) $\ell^{(i)}<\ell, \ell_{(i+1)} \leq \ell+1<\ell_{(i)}=\infty$ and $\tilde{f}_{i}$ acting on $(\nu, J)$ creates a singular string.

Proof. In Section 5, we analyze all possible cases such that $\tilde{f}_{i}(\nu, J) \neq 0$. Then only cases such that $\tilde{f}_{i}(\nu, J)$ is defined and $\widetilde{f}_{i}(\bar{\nu}, \bar{J})$ is undefined are the above two cases as described in Proposition 5.6 and Proposition 5.51. 


\section{Lemma 6.3.}

(1) Suppose that $\ell^{(i-1)} \leq \ell+1<\ell^{(i)}=\infty$ and $\widetilde{f}_{i}$ acting on $(\nu, J)$ creates a singular string. Then we have $\widetilde{\ell}^{(i)}=\ell+1, \widetilde{\ell}^{(i+1)}=\infty$ and $P_{\ell}^{(i)}(\bar{\nu})=x_{\ell}$.

(2) Suppose that $\ell^{(i)}<\ell, \ell_{(i+1)} \leq \ell+1<\ell_{(i)}=\infty$ and $\tilde{f}_{i}$ acting on $(\nu, J)$ creates a singular string. Then we have $\tilde{\ell}_{(i)}=\ell+1, \tilde{\ell}_{(i-1)}=\infty$ and $P_{\ell}^{(i)}(\bar{\nu})=x_{\ell}$.

Proof. (1) To begin with we shall show that there is no string of $\bar{\nu}^{(i)}$ that is longer than $\ell$. Suppose if possible that such string exist. Let $j$ be the minimal integer satisfying $j>\ell$ and $m_{j}^{(i)}(\nu)>0$. Then there are strings $\left(\ell, x_{\ell}\right)$ and $\left(j, x_{j}\right)$ of $(\nu, J)^{(i)}$ that satisfy $x_{j}>x_{\ell}$ by definition of $\ell$. Since $\ell^{(i)}=\infty$ we have $(\bar{\nu}, \bar{J})^{(i)}=(\nu, J)^{(i)}$. Thus the string $\left(j, x_{j}\right)$ still exists in $(\bar{\nu}, \bar{J})^{(i)}$, which implies that $P_{j}^{(i)}(\bar{\nu}) \geq x_{j}$. To summarize, we obtain $P_{j}^{(i)}(\bar{\nu})>x_{\ell}$.

Consider the case $j=\ell+1$. Then this result is in contradiction to the equality $P_{\ell+1}^{(i)}(\bar{\nu})=x_{\ell}$ that appears in the proof of Proposition 5.6. Next consider the general case $j>\ell+1$. By the convexity relation between $\ell$ and $j$, we have $P_{\ell+1}^{(i)}(\bar{\nu}) \geq \min \left\{P_{\ell}^{(i)}(\bar{\nu}), P_{j}^{(i)}(\bar{\nu})\right\}$. Since we know that $P_{\ell+1}^{(i)}(\bar{\nu})=x_{\ell}<P_{j}^{(i)}(\bar{\nu})$, we deduce that $P_{\ell+1}^{(i)}(\bar{\nu}) \geq P_{\ell}^{(i)}(\bar{\nu})$. From $P_{\ell+1}^{(i)}(\bar{\nu})=x_{\ell}$ and $P_{\ell}^{(i)}(\bar{\nu}) \geq x_{\ell}\left(\right.$ since $\left.\ell^{(i)}=\infty\right)$, we obtain $P_{\ell}^{(i)}(\bar{\nu})=P_{\ell+1}^{(i)}(\bar{\nu})=x_{\ell}$. Then the convexity relation between $\ell$ and $j$ gives $x_{\ell}=P_{\ell}^{(i)}(\bar{\nu}) \geq P_{j}^{(i)}(\bar{\nu})$ which is in contradiction to the relation $P_{j}^{(i)}(\bar{\nu})>x_{\ell}$. Hence there is no string of $\bar{\nu}^{(i)}$ that is longer than $\ell$.

The relations $P_{\ell}^{(i)}(\bar{\nu}) \geq x_{\ell}, P_{\ell+1}^{(i)}(\bar{\nu})=x_{\ell}, P_{\infty}^{(i)}(\bar{\nu})>-\infty$ and the convexity relation between $\ell$ and $\infty$ gives $P_{\ell}^{(i)}(\bar{\nu})=P_{\ell+1}^{(i)}(\bar{\nu})=P_{\ell+2}^{(i)}(\bar{\nu})=\cdots$. This relation forces $m_{k}^{(i+1)}(\bar{\nu})=0$ for all $k \geq \ell+1$, since otherwise the relation would be strictly convex. Since $\ell^{(i)}=\infty$, we have $\bar{\nu}^{(i+1)}=\nu^{(i+1)}$ and thus $\bar{\nu}^{(i+1)}=\widetilde{\nu}^{(i+1)}$. Therefore we obtain $m_{k}^{(i+1)}(\widetilde{\nu})=0$ for all $k \geq \ell+1$.

Recall that the definition of $\tilde{f}_{i}$ on the rigged configurations implies that $\tilde{\ell}^{(a)}=\ell^{(a)}$ for all $a \leq i-1$. Then by the assumption we have $\widetilde{\ell}^{(i-1)} \leq \ell+1$. Again from the assumption there is a length $\ell+1$ singular string in $\widetilde{\nu}^{(i)}$. Therefore we have $\widetilde{\ell}^{(i)}=\ell+1$. Finally from $m_{k}^{(i+1)}(\widetilde{\nu})=0$ for all $k \geq \ell+1$ we have $\widetilde{\ell}^{(i+1)}=\infty$.

(2) During the proof of Proposition 5.51 we show $P_{\ell+1}^{(i)}(\bar{\nu})=x_{\ell}$. Then we can use the same arguments of (1) if we replace $\ell^{(i-1)}, \ell^{(i)}$ and $\ell^{(i+1)}$ etc. there by $\ell_{(i+1)}, \ell_{(i)}$ and $\ell_{(i-1)}$ etc., respectively. Note that in this case we have to work under the condition $\ell^{(i)}<\ell$ and $\ell_{(i)}=\infty$. Then we can use the fact that all strings of $(\nu, J)^{(i-1)}$ that are strictly longer than $\ell^{(i-1)}(<\ell+1)$ do not change after $\delta$. In particular, we can show $m_{k}^{(i-1)}(\bar{\nu})=m_{k}^{(i-1)}(\widetilde{\nu})=0$ for all $k \geq \ell+1$.

Proof of Proposition 6.1. According to the previous observations, we have to consider two distinct cases.

(1) Suppose that $\ell^{(i-1)} \leq \ell+1<\ell^{(i)}=\infty$ and $\widetilde{f}_{i}$ acting on $(\nu, J)$ creates a singular string. From $\ell^{(i-1)}<\infty$ and $\ell^{(i)}=\infty$ we have $\Phi(\nu, J)=i \otimes b^{\prime}$. From $\widetilde{\ell}^{(i)}<\infty$ and $\widetilde{\ell}^{(i+1)}=\infty$ we have $\Phi(\widetilde{\nu}, \widetilde{J})=\Phi \circ \widetilde{f}_{i}(\nu, J)=(i+1) \otimes b^{\prime \prime}$. Let us show the coincidence $b^{\prime \prime}=b^{\prime}$ by proving $(\bar{\nu}, \bar{J})=(\overline{\widetilde{\nu}}, \widetilde{\widetilde{J}})$. Recall that the difference between $(\nu, J)$ and $(\widetilde{\nu}, \widetilde{J})$ is one box at $(\ell+1)$-th column of $(\widetilde{\nu}, \widetilde{J})^{(i)}$. Then from $\widetilde{\ell}^{(a)}=\ell^{(a)}$ for all $a \leq i-1, \widetilde{\ell}^{(i)}=\ell+1$ and $\widetilde{\ell}^{(i+1)}=\ell^{(i)}=\infty$, we have $\bar{\nu}=\overline{\widetilde{\nu}}$. In order to show $\bar{J}=\overline{\widetilde{J}}$ recall that $\delta$ acting on $(\widetilde{\nu}, \widetilde{J})$ creates the length $\ell$ singular string of $(\overline{\widetilde{\nu}}, \overline{\widetilde{J}})^{(i)}$. On the other hand, since $P_{\ell}^{(i)}(\bar{\nu})=x_{\ell}$ all length $\ell$ strings of $(\bar{\nu}, \bar{J})^{(i)}$ are singular. From $P_{\ell}^{(i)}(\bar{\nu})=P_{\ell}^{(i)}(\overline{\widetilde{\nu}})$ we see the coincidence of the corresponding riggings and hence we deduce that $\bar{J}=\overline{\widetilde{J}}$. Thus we have $b^{\prime \prime}=b^{\prime}$.

As for $b^{\prime}=\Phi(\bar{\nu}, \bar{J})$, we know that $\varphi_{i}\left(b^{\prime}\right)=\varphi_{i}(\bar{\nu}, \bar{J})=0$ by the assumption. Thus $\tilde{f}_{i} \circ \Phi(\nu, J)=$ $\widetilde{f}_{i}(i) \otimes b^{\prime}=(i+1) \otimes b^{\prime}$. Thus $\Phi$ and $\widetilde{f}_{i}$ commutes in this case. 
(2) Suppose that $\ell^{(i)}<\ell, \ell_{(i+1)} \leq \ell+1<\ell_{(i)}=\infty$ and $\tilde{f}_{i}$ acting on $(\nu, J)$ creates a singular string. From $\ell_{(i+1)}<\infty$ and $\ell_{(i)}=\infty$ we have $\Phi(\nu, J)=\overline{i+1} \otimes b^{\prime}$. From $\tilde{\ell}_{(i)}<\infty$ and $\tilde{\ell}_{(i-1)}=\infty$ we have $\Phi(\widetilde{\nu}, \widetilde{J})=\bar{i} \otimes b^{\prime \prime}$. The coincidence $b^{\prime \prime}=b^{\prime}$ follows from parallel arguments. Finally, by $\varphi_{i}\left(b^{\prime}\right)=\varphi_{i}(\bar{\nu}, \bar{J})=0$ we have $\tilde{f}_{i} \circ \Phi(\nu, J)=\tilde{f}_{i}(\overline{i+1}) \otimes b^{\prime}=\bar{i} \otimes b^{\prime}$. Thus $\Phi$ and $\tilde{f}_{i}$ commutes in this case also.

\subsection{Proof for (2)}

To begin with, let us show the following property.

Lemma 6.4. Suppose that $\ell_{(i+1)}<\infty, \ell_{(i)}=\infty, \widetilde{\ell}_{(i)}<\infty$ and $\tilde{\ell}_{(i-1)}=\infty$. Let $s$ (resp. $\left.\bar{s}\right)$ be the smallest rigging of $(\nu, J)^{(i)}\left(\right.$ resp. $\left.(\bar{\nu}, \bar{J})^{(i)}\right)$. Then we have $s \leq \bar{s}$.

Proof. Note that if we show that the rigging for the shortened string in $(\bar{\nu}, \bar{J})^{(i)}$ is larger than or equal to $s$, then we have $s \leq \bar{s}$. In particular, we can assume that $\ell^{(i)}>1$, since otherwise the corresponding rigging will be erased by $\delta$ and will not contribute to $\bar{s}$, thus $s=\bar{s}$ by $\ell_{(i)}=\infty$. Since $\ell_{(i)}=\infty$, it is enough to check

$$
P_{\ell^{(i)}-1}^{(i)}(\bar{\nu}) \geq s .
$$

Then we have the two possibilities:

$$
\text { (I) } \ell^{(i-1)}=\ell^{(i)} \quad \text { or } \quad \text { (II) } \ell^{(i-1)}<\ell^{(i)} .
$$

During the proof, let $j$ be the largest integer such that $j<\ell^{(i)}$ and $m_{j}^{(i)}(\nu)>0$. If there is no such $j$, we set $j=0$.

Case (I). In this case we have to show that $P_{\ell^{(i)}-1}^{(i)}(\nu) \geq s$ under the assumption $\ell^{(i-1)}=\ell^{(i)}$. Let us consider the case $j=\ell^{(i)}-1$. Then we are done since we have $P_{\ell^{(i)}-1}^{(i)}(\nu) \geq x_{\ell^{(i)}-1} \geq s$ by the minimality of $s$. Next consider the case $0<j<\ell^{(i)}-1$. Then by the convexity relation between $j$ and $\ell^{(i)}$ we have $P_{\ell^{(i)}-1}^{(i)}(\nu) \geq \min \left\{P_{j}^{(i)}(\nu), P_{\ell^{(i)}}^{(i)}(\nu)\right\} \geq s$. Suppose that $j=0$. If $s \leq 0$, we can use the same arguments of the previous case since $P_{0}^{(i)}(\nu)=0$.

Therefore we have to consider the case $j=0$ and $s>0$. Since $s>0, \widetilde{f}_{i}$ acting on $(\nu, J)$ adds the length 1 string to $(\nu, J)^{(i)}$. Recall that $\tilde{f}_{i}$ does not change coriggings of untouched strings. Since we have $\ell^{(i-1)}=\ell^{(i)}>1$, we see that $\widetilde{\ell}^{(a)}=\ell^{(a)}$ and $\widetilde{\ell}_{(a)}=\ell_{(a)}$ for all $a$. However this is in contradiction to the assumptions $\ell_{(i)}=\infty$ and $\tilde{\ell}_{(i)}<\infty$. Hence this case cannot happen.

Case (II). In this case we have to show that $P_{\ell^{(i)}-1}^{(i)}(\nu)>s$ under the assumption $\ell^{(i-1)}<\ell^{(i)}$. Suppose if possible that $P_{\ell^{(i)}-1}^{(i)}(\nu) \leq s$.

Suppose that $j=\ell^{(i)}-1$. Then we have $x_{\ell^{(i)}-1} \leq P_{\ell^{(i)}-1}^{(i)}(\nu) \leq s$. Then by the minimality of $s$, we have $x_{\ell^{(i)}-1}=P_{\ell^{(i)}-1}^{(i)}(\nu)=s$, in particular, the string $\left(\ell^{(i)}-1, x_{\ell^{(i)}-1}\right)$ is singular. However this is a contradiction since its length satisfies $\ell^{(i-1)} \leq \ell^{(i)}-1<\ell^{(i)}$.

Suppose that $\ell^{(i-1)} \leq j<\ell^{(i)}-1$. By definition of $s$ we have $P_{\ell^{(i)}}^{(i)}(\nu) \geq s$ and by the assumption we have $P_{\ell^{(i)}-1}^{(i)}(\nu) \leq s$. Then by the convexity relation of $P_{k}^{(i)}(\nu)$ between $j \leq k \leq$ $\ell^{(i)}$, we have $P_{\ell^{(i)}}^{(i)}(\nu) \geq P_{\ell^{(i)}-1}^{(i)}(\nu) \geq P_{j}^{(i)}(\nu) \geq x_{j}$. By the minimality of $s$ and $s \geq P_{\ell^{(i)}-1}^{(i)}(\nu)$, we have $P_{j}^{(i)}(\nu)=x_{j}$, that is, the string $\left(j, x_{j}\right)$ is singular. This contradicts the definition of $\ell^{(i)}$.

Suppose that $0<j<\ell^{(i-1)}$. By $m_{\ell^{(i-1)}}^{(i-1)}(\nu)>0$, the convexity relation of $P_{k}^{(i)}(\nu)$ between $\ell^{(i-1)}+1 \geq k \geq \ell^{(i-1)}-1$ has to be strictly convex. Therefore we have $P_{\ell^{(i)}}^{(i)}(\nu) \geq P_{\ell^{(i)}-1}^{(i)}(\nu)>$ $P_{j}^{(i)}(\nu) \geq x_{j}$ in this case. Since we have $s \geq P_{\ell^{(i)}-1}^{(i)}(\nu)$, this contradicts the minimality of $s$. 
Suppose that $j=0$. Then we have $s \geq P_{\ell^{(i)}-1}^{(i)}(\nu)>P_{0}^{(i)}(\nu)=0$. If $s \leq 0$, this is a contradiction. Hence we assume that $s>0$. Then $\widetilde{f}_{i}$ adds the length 1 string $(1,-1)$ to $(\nu, J)^{(i)}$. If $\ell^{(i-1)}>1$, we can use the same arguments in the last part of Case (I) to show that such case cannot happen. Thus assume that $\ell^{(i-1)}=1$. If $P_{1}^{(i)}(\widetilde{\nu})>-1$, then the added string $(1,-1)$ is non-singular so that we can use the arguments in the last part of Case (I) to show that such case cannot happen.

To summarize, we have shown that we have $s \leq \bar{s}$ except for the case $j=0, s>0, \ell^{(i-1)}=1$ and $P_{1}^{(i)}(\widetilde{\nu})=-1$. Suppose if possible that this case happen. Since $s>0, \widetilde{f}_{i}$ adds the length 1 string $(1,-1)$ to $(\nu, J)^{(i)}$. Thus we have $\bar{s}<0$ and $P_{1}^{(i)}(\nu)=1$. Since $m_{\ell^{(i-1)}}^{(i-1)}(\nu)=m_{1}^{(i-1)}(\nu)>0$ and $\ell^{(i)}>1$, the relation $P_{k}^{(i)}(\nu)$ for $0 \leq k \leq 2$ must be strictly convex. In view of $P_{0}^{(i)}(\nu)=0$, this implies that $1=P_{1}^{(i)}(\nu) \geq P_{2}^{(i)}(\nu)$. Since we are assuming that $s>0$, the rigging of the string $\left(\ell^{(i)}, P_{\ell^{(i)}}^{(i)}(\nu)\right)$ must satisfy $P_{\ell^{(i)}}^{(i)}(\nu)>0$. Then by the convexity relation of $P_{k}^{(i)}(\nu)$ between $1 \leq k \leq \ell^{(i)}$ the only possibility that is compatible with $1=P_{1}^{(i)}(\nu) \geq P_{2}^{(i)}(\nu)$ is the case $1=P_{1}^{(i)}(\nu)=P_{2}^{(i)}(\nu)=\cdots=P_{\ell^{(i)}}^{(i)}(\nu)$. To summarize, we have the following situation:

$$
s>0, \ell^{(i-1)}=1, \ell^{(i)}>1 \quad \text { and } \quad P_{1}^{(i)}(\nu)=P_{2}^{(i)}(\nu)=\cdots=P_{\ell^{(i)}}^{(i)}(\nu)=1 .
$$

Since $j=0$ we have $m_{1}^{(i)}(\nu)=0$. Then from the convexity relation of Lemma 3.13 with $l=1$ we have

$$
\begin{aligned}
& -P_{0}^{(i)}(\nu)+2 P_{1}^{(i)}(\nu)-P_{2}^{(i)}(\nu)=1 \\
& \quad \geq m_{1}^{(i-1)}(\nu)-2 m_{1}^{(i)}(\nu)+m_{1}^{(i+1)}(\nu)=m_{1}^{(i-1)}(\nu)+m_{1}^{(i+1)}(\nu) .
\end{aligned}
$$

We have $m_{1}^{(i-1)}(\nu) \geq 1$ since $\ell^{(i-1)}=1$ and $m_{1}^{(i+1)}(\nu) \geq 0$ by definition. From the above inequality we conclude that $m_{1}^{(i-1)}(\nu)=1$ and $m_{1}^{(i+1)}(\nu)=0$. We also have $m_{k}^{(i+1)}(\nu)=0$ for $1<k<\ell^{(i)}$ by the relation $P_{1}^{(i)}(\nu)=P_{2}^{(i)}(\nu)=\cdots=P_{\ell^{(i)}}^{(i)}(\nu)$. Since $\widetilde{f}_{i}$ does not change the coriggings of $(\nu, J)^{(a)}$ for $a<i$, we have $\widetilde{\ell}^{(i-1)}=\ell^{(i-1)}=1$. Recall that the string $(1,-1)$ of $(\widetilde{\nu}, \widetilde{J})^{(i)}$ is singular. Thus $\widetilde{\ell}^{(i)}=1$. From $\widetilde{\nu}^{(i+1)}=\nu^{(i+1)}$, we have $m_{k}^{(i+1)}(\widetilde{\nu})=0$ for $1 \leq k<\ell^{(i)}$. Since $\widetilde{f}_{i}$ does not change the coriggings of $(\nu, J)^{(i+1)}$, we have $\widetilde{\ell}^{(i+1)}=\ell^{(i+1)}$, and thus $\widetilde{\ell}^{(a)}=\ell^{(a)}$ for all $i<a$ and $\widetilde{\ell}_{(a)}=\ell_{(a)}$ for all $a$. In particular we have $\widetilde{\ell}_{(i)}=\ell_{(i)}=\infty$. However this is a contradiction since we have $\tilde{\ell}_{(i)}<\infty$ by the assumption. Thus the situation (6.1) cannot happen.

Proposition 6.5. Let $b \in B^{1,1} \otimes \bar{B}$ and suppose that we have shown the commutativity of $\widetilde{f}_{i}$ and $\Phi$ for the elements of $\bar{B}$. Suppose that we have ${\widetilde{f_{i}}}_{\tilde{f}}(b) \neq 0$ and $\widetilde{f}_{i}\left(b^{\prime}\right)=0$ where $\underline{\tilde{f}}^{\prime} \in \bar{B}$ is the corresponding part of $b$. Then we have $\widetilde{f}_{i}(\nu, J) \neq 0, \widetilde{f}_{i}(\bar{\nu}, \bar{J})=0$ and $\Phi^{-1}\left(\widetilde{f}_{i}(b)\right)=\widetilde{f}_{i}\left(\Phi^{-1}(b)\right)$.

Proof. By the assumption, we have the following two possibilities:

(a) $b=i \otimes b^{\prime}$ and $\widetilde{f}_{i}(b)=(i+1) \otimes b^{\prime}$,

(b) $b=\overline{i+1} \otimes b^{\prime}$ and $\tilde{f}_{i}(b)=\bar{i} \otimes b^{\prime}$

Case (a). In this case we have $\ell^{(i-1)}<\infty$ and $\ell^{(i)}=\infty$. Then we have $P_{\infty}^{(i)}(\nu)=P_{\infty}^{(i)}(\bar{\nu})+1$. By the induction hypothesis, $\widetilde{f}_{i}\left(b^{\prime}\right)=0$ implies that $\varphi_{i}(\bar{\nu}, \bar{J})=0$. Then from Theorem 3.8 we have $P_{\infty}^{(i)}(\bar{\nu})=\bar{s}$ where $\bar{s}$ is the smallest rigging of $(\bar{\nu}, \bar{J})^{(i)}$. Let $s$ be the smallest rigging of $(\nu, J)^{(i)}$. Since $\ell^{(i)}=\infty$, we have $s=\bar{s}$. Again by Theorem 3.8 we have $\varphi_{i}(\nu, J)=P_{\infty}^{(i)}(\nu)-s=$ $P_{\infty}^{(i)}(\bar{\nu})+1-s=\bar{s}+1-s=1$. Therefore $\widetilde{f}_{i}(\nu, J) \neq 0$ as desired. 
In order to check $\Phi^{-1}\left(\widetilde{f}_{i}(b)\right)=\widetilde{f}_{i}\left(\Phi^{-1}(b)\right)$, it is enough to show that $\widetilde{f}_{i}$ acting on $(\nu, J)$ creates a singular string and it satisfies $\widetilde{\ell}^{(i-1)} \leq \ell+1=\widetilde{\ell}^{(i)}<\widetilde{\ell}^{(i+1)}=\infty$ under the assumptions $\widetilde{f}_{i}(\nu, J) \neq 0$ and $\widetilde{f}_{i}(\bar{\nu}, \bar{J})=0$. Then this is the consequence of Proposition 6.2.

Case (b). In this case we have $\ell_{(i+1)}<\infty, \ell_{(i)}=\infty, \widetilde{\ell}_{(i)}<\infty$ and $\widetilde{\ell}_{(i-1)}=\infty$. By $\ell_{(i+1)}<\infty$ and $\ell_{(i)}=\infty$ we have $P_{\infty}^{(i)}(\nu)=P_{\infty}^{(i)}(\bar{\nu})+1$. Since we have $\varphi_{i}(\bar{\nu}, \bar{J})=0$ by $\widetilde{f}_{i}\left(b^{\prime}\right)=0$, Theorem 3.8 asserts that $P_{\infty}^{(i)}(\bar{\nu})=\bar{s}$. Now let us invoke the relation $s \leq \bar{s}$ of Lemma 6.4. Then we have $\varphi_{i}(\nu, J)=P_{\infty}^{(i)}(\nu)-s=P_{\infty}^{(i)}(\bar{\nu})+1-s=\bar{s}-s+1 \geq 1$. Thus $\widetilde{f}_{i}(\nu, J) \neq 0$.

Finally we can check $\Phi^{-1}\left(\widetilde{f}_{i}(b)\right)=\widetilde{f}_{i}\left(\Phi^{-1}(b)\right)$ under the assumptions $\tilde{f}_{i}(\nu, J) \neq 0$ and $\widetilde{f}_{i}(\bar{\nu}, \bar{J})=0$ by using Proposition 6.2 .

\subsection{Proof for (3)}

For the analysis of the present situation, let us prepare several properties of the rigged configurations. Let $s$ (resp. $\bar{s})$ be the smallest rigging of $(\nu, J)^{(i)}\left(\operatorname{resp} .(\bar{\nu}, \bar{J})^{(i)}\right)$.

Lemma 6.6. Suppose that we have $\widetilde{f}_{i}(\nu, J)=0$ and $\widetilde{f}_{i}(\bar{\nu}, \bar{J}) \neq 0$. Then we have the following two possibilities:

(1) $\ell^{(i)}<\infty$ and $\ell^{(i+1)}=\infty$, or

(2) $\ell_{(i)}<\infty$ and $\ell_{(i-1)}=\infty$.

Proof. Let us show that $\ell^{(i)}<\infty$. Suppose if possible that $\ell^{(i)}=\infty$. From Theorem 3.8 we have $\varphi_{i}(\nu, J)=P_{\infty}^{(i)}(\nu)-s=0$. Since $\ell^{(i)}=\infty$, we have $s=\bar{s}$ and $P_{\infty}^{(i)}(\bar{\nu}) \leq P_{\infty}^{(i)}(\nu)$. Thus $\varphi_{i}(\bar{\nu}, \bar{J})=P_{\infty}^{(i)}(\bar{\nu})-s \leq 0$ contradicting the assumption $\widetilde{f}_{i}(\bar{\nu}, \bar{J}) \neq 0$. Hence we conclude that $\ell^{(i)}<\infty$.

Suppose that $\ell^{(i+1)}<\infty$. Then we have the following four possibilities:

(a) $\ell_{(i+1)}=\infty$,

(b) $\ell_{(i+1)}<\infty$ and $\ell_{(i)}=\infty$,

(c) $\ell_{(i)}<\infty$ and $\ell_{(i-1)}=\infty$,

(d) $\ell_{(i-1)}<\infty$.

Let us analyze these possibilities case by case. Note that by the assumption $\tilde{f}_{i}(\nu, J)=0$, we have $\varphi_{i}(\nu, J)=P_{\infty}^{(i)}(\nu)-s=0$ from Theorem 3.8.

Case (a). By the assumptions $\ell^{(i+1)}<\infty$ and $\ell_{(i+1)}=\infty$, we have $P_{\infty}^{(i)}(\bar{\nu})=P_{\infty}^{(i)}(\nu)$. Then the assumption $\widetilde{f}_{i}(\bar{\nu}, \bar{J}) \neq 0$ implies that

$$
\varphi_{i}(\bar{\nu}, \bar{J})=P_{\infty}^{(i)}(\bar{\nu})-\bar{s}=P_{\infty}^{(i)}(\nu)-\bar{s}=s-\bar{s}>0 .
$$

By the assumption $\ell_{(i+1)}=\infty$, we see that $s>\bar{s}$ can happen only if the string $\left(\ell^{(i)}-1, P_{\ell^{(i)}-1}^{(i)}(\bar{\nu})\right)$ which is created by $\delta$ has the rigging strictly smaller than $s$. Thus the present assumptions imply that $P_{\ell^{(i)}-1}^{(i)}(\bar{\nu})<s$. To summarize we are left with the following two possibilities:

(I) $\ell^{(i-1)}=\ell^{(i)}$, then $P_{\ell^{(i)}-1}^{(i)}(\nu)<s$ by $P_{\ell^{(i)}-1}^{(i)}(\bar{\nu})=P_{\ell^{(i)}-1}^{(i)}(\nu)$,

(II) $\ell^{(i-1)}<\ell^{(i)}$, then $P_{\ell^{(i)}-1}^{(i)}(\nu) \leq s$ by $P_{\ell^{(i)}-1}^{(i)}(\bar{\nu})=P_{\ell^{(i)}-1}^{(i)}(\nu)-1$.

Let us show that these cases cannot happen. Let $j$ be the largest integer such that $j<\ell^{(i)}$ and $m_{j}^{(i)}(\nu)>0$. If there is no such $j$, set $j=0$. 
Case (a-I). Suppose that $j=\ell^{(i)}-1$. Then we have $x_{j} \leq P_{j}^{(i)}(\nu)<s$, which contradicts the minimality of $s$. Suppose that $0<j<\ell^{(i)}-1$. Recall that by the minimality of $s$ we have $P_{\ell^{(i)}}^{(i)}(\nu) \geq s$. Thus we have $P_{\ell^{(i)}}^{(i)}(\nu)>P_{\ell^{(i)}-1}^{(i)}(\nu)$. Then by the convexity relation of $P_{k}^{(i)}(\nu)$ between $j \leq k \leq \ell^{(i)}$ we have $P_{\ell^{(i)}}^{(i)}(\nu)>P_{\ell^{(i)}-1}^{(i)}(\nu)>P_{j}^{(i)}(\nu) \geq x_{j}$. Since we are assuming that $s>P_{\ell^{(i)}-1}^{(i)}(\nu)$, this contradicts the minimality of $s$.

Finally suppose that $j=0$. Then the previous inequalities become $s>P_{\ell^{(i)}-1}^{(i)}(\nu)>P_{0}^{(i)}(\nu)=0$. Note that this relation is always valid since we have $\ell^{(i)} \geq 1$ by the definition. Therefore we have $s>0$ which implies that $\widetilde{f}_{i}$ acting on $(\nu, J)$ adds the length 1 string $(1,-1)$ to $(\nu, J)^{(i)}$. Since we are assuming that $\widetilde{f}_{i}(\nu, J)=0$, the rigging of the string $(1,-1)$ of $(\widetilde{\nu}, \widetilde{J})^{(i)}$ must be larger than the corresponding vacancy number. Thus we have $P_{1}^{(i)}(\widetilde{\nu})<-1$, that is, $P_{1}^{(i)}(\nu) \leq 0$ by $P_{1}^{(i)}(\widetilde{\nu})=P_{1}^{(i)}(\nu)-2$. Since $P_{0}^{(i)}(\nu)=0$, the convexity relation of $P_{k}^{(i)}(\nu)$ between $0 \leq k \leq \ell^{(i)}$ implies that $0=P_{0}^{(i)}(\nu) \geq P_{1}^{(i)}(\nu) \geq P_{\ell^{(i)}}^{(i)}(\nu)$. Therefore the rigging of the string $\left(\ell^{(i)}, P_{\ell^{(i)}}^{(i)}(\nu)\right)$ of $(\nu, J)^{(i)}$ satisfies that $P_{\ell^{(i)}}^{(i)}(\nu) \leq 0$. This is a contradiction since we have $s>0$.

Case (a-II). Suppose that $j=\ell^{(i)}-1$. Then we have $x_{j} \leq P_{j}^{(i)}(\nu) \leq s$. By the minimality of $s$ we have $x_{j}=P_{j}^{(i)}(\nu)=s$, in particular, the string $\left(j, x_{j}\right)$ of $(\nu, J)^{(i)}$ is singular. However this is in contradiction to the definition of $\ell^{(i)}$ since we have $\ell^{(i-1)} \leq j<\ell^{(i)}$. Suppose that $\ell^{(i-1)} \leq j<\ell^{(i)}-1$. Then by the convexity relation of $P_{k}^{(i)}(\nu)$ between $j \leq k \leq \ell^{(i)}$ we have $s \geq P_{\ell^{(i)}-1}^{(i)}(\nu) \geq P_{j}^{(i)}(\nu) \geq x_{j}$. Then by the minimality of $s$ we have $P_{j}^{(i)}(\nu)=x_{j}$, in particular, the string $\left(j, x_{j}\right)$ of $(\nu, J)^{(i)}$ is singular. Again this is in contradiction to the definition of $\ell^{(i)}$ since $\ell^{(i-1)} \leq j<\ell^{(i)}$. Suppose that $0<j<\ell^{(i-1)}$. Since $m_{\ell^{(i-1)}}^{(i-1)}(\nu)>0$, the convexity relation of $P_{k}^{(i)}(\nu)$ between $\ell^{(i-1)}-1 \leq k \leq \ell^{(i-1)}+1$ is strictly convex. Therefore we have $s \geq P_{\ell^{(i)}-1}^{(i)}(\nu)>P_{j}^{(i)}(\nu) \geq x_{j}$. This contradicts the minimality of $s$.

Finally suppose that $j=0$. Then the above relation becomes $s \geq P_{\ell^{(i)}-1}^{(i)}(\nu)>P_{0}^{(i)}(\nu)=0$, that is, $s>0$. By the similar arguments of the latter part of Case (a-I), we obtain $P_{1}^{(i)}(\nu) \leq 0$. Since $P_{0}^{(i)}(\nu)=0$ and $m_{\ell^{(i-1)}}^{(i-1)}(\nu)>0$, the convexity relation of $P_{k}^{(i)}(\nu)$ between $0 \leq k \leq \ell^{(i)}$ becomes $0=P_{0}^{(i)}(\nu) \geq P_{\ell^{(i-1)}}^{(i)}(\nu)>P_{\ell^{(i)}}^{(i)}(\nu)$. Hence the rigging of the string $\left(\ell^{(i)}, P_{\ell^{(i)}}^{(i)}(\nu)\right)$ of $(\nu, J)^{(i)}$ satisfies that $P_{\ell^{(i)}}^{(i)}(\nu)<0$. This is a contradiction since we have $s>0$.

Case (b). By the assumptions $\ell_{(i+1)}<\infty$ and $\ell_{(i)}=\infty$, we have $P_{\infty}^{(i)}(\bar{\nu})=P_{\infty}^{(i)}(\nu)-1$. Then the assumption $\widetilde{f}_{i}(\bar{\nu}, \bar{J}) \neq 0$ implies that

$$
\varphi_{i}(\bar{\nu}, \bar{J})=P_{\infty}^{(i)}(\bar{\nu})-\bar{s}=P_{\infty}^{(i)}(\nu)-1-\bar{s}=s-\bar{s}-1>0 .
$$

By the assumption $\ell_{(i)}=\infty$, the situation $s-1>\bar{s}$ can happen only if the rigging of the string $\left(\ell^{(i)}-1, P_{\ell^{(i)}-1}^{(i)}(\bar{\nu})\right)$ which is created by $\delta$ satisfies $P_{\ell^{(i)}-1}^{(i)}(\bar{\nu})<s-1$. Then we can use the same arguments of the above Case (a) to show that this case cannot happen.

Case $(\mathbf{c})$. By the assumptions $\ell_{(i)}<\infty$ and $\ell_{(i-1)}=\infty$, we have $P_{\infty}^{(i)}(\bar{\nu})=P_{\infty}^{(i)}(\nu)+1$. Then the assumption $\widetilde{f}_{i}(\bar{\nu}, \bar{J}) \neq 0$ implies that

$$
\varphi_{i}(\bar{\nu}, \bar{J})=P_{\infty}^{(i)}(\bar{\nu})-\bar{s}=P_{\infty}^{(i)}(\nu)+1-\bar{s}=s-\bar{s}+1>0 \quad \Longleftrightarrow \quad s \geq \bar{s} .
$$

Thus, basically we have the following two cases:

$$
\text { (1) } s>\bar{s}, \quad(2) \quad s=\bar{s} .
$$

Case (c-1) To begin with let us consider the case $s>\bar{s}$. This situation can happen if at least one of the riggings for the strings $\left(\ell^{(i)}-1, P_{\ell^{(i)}-1}^{(i)}(\bar{\nu})\right)$ or $\left(\ell_{(i)}-1, P_{\ell_{(i)}-1}^{(i)}(\bar{\nu})\right)$ is smaller 
than $s$. If $P_{\ell^{(i)}-1}^{(i)}(\bar{\nu})<s$, we can use the same arguments of Case (a) to show that this case cannot happen. Thus $P_{\ell^{(i)}-1}^{(i)}(\bar{\nu}) \geq s>\bar{s}$.

Therefore we suppose that $P_{\ell^{(i)}-1}^{(i)}(\bar{\nu})>\bar{s}$ and $P_{\ell_{(i)}-1}^{(i)}(\bar{\nu})=\bar{s}$ in the sequel. Let us follow the classification (III) to (VII) of Lemma 5.1. We observe that the Cases (III) and (IV) cannot happen in this setting, since in these settings we have $\ell^{(i)}=\ell_{(i)}$. This is a contradiction since we are assuming that $P_{\ell^{(i)}-1}^{(i)}(\bar{\nu})>P_{\ell_{(i)}-1}^{(i)}(\bar{\nu})$. Let us consider the remaining cases under the assumption $\ell^{(i)}<\ell_{(i)}$.

Case $(\mathbf{c}-\mathbf{1 - V})$. In this case we have $P_{\ell_{(i)}-1}^{(i)}(\nu)=\bar{s}-1$. Let $j$ be the largest integer such that $j<\ell_{(i)}$ and $m_{j}^{(i)}(\nu)>0$. Since $\ell^{(i)}<\ell_{(i)}$ we have $\ell^{(i)} \leq j$. If $j=\ell_{(i)}-1$, we obtain the immediate contradiction $s>\bar{s}-1=P_{\ell_{(i)}-1}^{(i)}(\nu) \geq x_{\ell_{(i)}-1}$. Thus suppose that $\ell^{(i)} \leq j<\ell_{(i)}-1$. Since $s$ is the minimal rigging of $(\nu, J)^{(i)}$ we see that $P_{\ell^{(i)}}^{(i)}(\nu) \geq s>\bar{s}$. Therefore we have $P_{\ell^{(i)}}^{(i)}(\nu)>P_{\ell^{(i)}-1}^{(i)}(\nu)$. Then by the convexity relation of $P_{k}^{(i)}(\nu)$ between $j \leq k \leq \ell^{(i)}$ we obtain $P_{\ell^{(i)}-1}^{(i)}(\nu)>P_{j}^{(i)}(\nu) \geq x_{j}$, in particular, $s>x_{j}$. This contradicts the minimality of $s$.

Case (c-1-VI). In this case we have $P_{\ell_{(i)}}^{(i)}(\nu)=\bar{s}$. Then we can use the same arguments of Case $(\mathrm{c}-1-\mathrm{V})$ to show that this case cannot happen.

Case (c-1-VII). In this case we have $P_{\ell_{(i)}-1}^{(i)}(\nu)=\bar{s}+1$ under the assumption $\ell_{(i+1)}<\ell_{(i)}$. If $\bar{s}+1<s$ we can use the same arguments of Case (c-1-V) to show that such case cannot happen. Thus suppose that $\bar{s}+1=s$. Let $j$ be the largest integer such that $j<\ell_{(i)}$ and $m_{j}^{(i)}(\nu)>0$. Since $\ell^{(i)}<\ell_{(i)}$ we have $\ell^{(i)} \leq j$.

Suppose that $j=\ell_{(i)}-1$. Then we see that the string $\left(\ell_{(i)}-1, x_{\ell_{(i)}-1}\right)$ is singular by the minimality of $s$, since we have $s=P_{\ell_{(i)}-1}^{(i)}(\nu) \geq x_{\ell_{(i)}-1}$. However this is in contradiction to the definition of $\ell_{(i)}$ since its length satisfies that $\ell_{(i+1)} \leq \ell_{(i)}-1<\ell_{(i)}$.

Suppose that $\ell_{(i+1)} \leq j<\ell_{(i)}-1$. Since $s$ is the minimal rigging of $(\nu, J)^{(i)}$, we have $P_{\ell_{(i)}}^{(i)}(\nu) \geq s$ and $P_{j}^{(i)}(\nu) \geq s$. Since we have $P_{\ell_{(i)}-1}^{(i)}(\nu)=s$, the only possibility that is compatible with the convexity relation of $P_{k}^{(i)}(\nu)$ between $j \leq k \leq \ell_{(i)}$ is the case $s=P_{\ell_{(i)}}^{(i)}(\nu)=P_{\ell_{(i)}-1}^{(i)}(\nu)=$ $\cdots=P_{j}^{(i)}(\nu) \geq x_{j}$. By the minimality of $s$ we have $P_{j}^{(i)}(\nu)=x_{j}$, in particular, the $\operatorname{string}\left(j, x_{j}\right)$ is singular. However this is in contradiction to the definition of $\ell_{(i)}$ since we have $\ell_{(i+1)} \leq j<\ell_{(i)}$.

Suppose that $\ell^{(i)} \leq j<\ell_{(i+1)}$. As in the previous paragraph, we obtain $s=P_{\ell_{(i)}}^{(i)}(\nu)=$ $P_{\ell_{(i)}-1}^{(i)}(\nu)=\cdots=P_{j}^{(i)}(\nu) \geq x_{j}$. However, this is a contradiction since we have $m_{\ell_{(i+1)}}^{(i+1)}(\nu)>0$, which implies that the vacancy numbers $P_{k}^{(i)}(\nu)$ are strictly convex function of $k$.

To summarize, we have shown that Case (c-1) cannot happen.

Case (c-2). In this case we have $s=\bar{s}$. This case is indeed possible as the example at the end of this subsection shows.

Case (d). By the assumption $\ell_{(i-1)}<\infty$, we have $P_{\infty}^{(i)}(\bar{\nu})=P_{\infty}^{(i)}(\nu)$. Then the assumptions $\tilde{f}_{i}(\bar{\nu}, \bar{J}) \neq 0$ and $\widetilde{f}_{i}(\nu, J)=0$ imply that

$$
\varphi_{i}(\bar{\nu}, \bar{J})=P_{\infty}^{(i)}(\bar{\nu})-\bar{s}=P_{\infty}^{(i)}(\nu)-\bar{s}=s-\bar{s}>0 \quad \Longleftrightarrow \quad s>\bar{s} .
$$

We can use the same arguments of Case (c-1) to show that this case cannot happen. 
Lemma 6.7. Suppose that we have $\widetilde{f}_{i}(\nu, J)=0$ and $\widetilde{f}_{i}(\bar{\nu}, \bar{J}) \neq 0$. Then we have the following two possibilities:

(1) $\ell^{(i)}<\infty, \ell^{(i+1)}=\infty$ and $s=\bar{s}$,

(2) $\ell_{(i)}<\infty, \ell_{(i-1)}=\infty$ and $s=\bar{s}$.

Proof. (1) Step 1. Let us show $s \leq \bar{s}$. It is enough to show that the new string of $(\bar{\nu}, \bar{J})^{(i)}$ has the rigging larger than or equal to $s$, that is, $P_{\ell^{(i)}-1}^{(i)}(\bar{\nu}) \geq s$. Suppose that $P_{\ell^{(i)}-1}^{(i)}(\bar{\nu})<s$. Then we can use the same arguments of Case (a) of the proof of Lemma 6.6 to show that this case cannot happen. Thus $s \leq \bar{s}$.

Step 2. Next, let us show $s \geq \bar{s}$. Then it is enough to show $P_{\ell^{(i)}}^{(i)}(\nu) \geq \bar{s}$ since the only rigging that is changed by $\delta^{-1}$ is the one associated with the lengthened string (see Remark 3.16 for the explanation of $\left.\delta^{-1}\right)$. By the assumption $\ell^{(i+1)}=\infty$ we have $P_{\ell^{(i)}}^{(i)}(\nu)=P_{\ell^{(i)}}^{(i)}(\bar{\nu})-1$. Thus the above relation is equivalent to $P_{\ell^{(i)}}^{(i)}(\bar{\nu})>\bar{s}$.

Suppose if possible that $P_{\ell^{(i)}}^{(i)}(\bar{\nu}) \leq \bar{s}$. Suppose if possible that there are strings of $(\bar{\nu}, \bar{J})^{(i)}$ that are longer than or equal to $\ell^{(i)}$. Let $j$ be the length of the shortest such string. By the minimality of $\bar{s}$ we have $P_{\ell^{(i)}-1}^{(i)}(\bar{\nu}) \geq \bar{s}$. Then we have $P_{\ell^{(i)}-1}^{(i)}(\bar{\nu}) \geq P_{\ell^{(i)}}^{(i)}(\bar{\nu}) \geq P_{j}^{(i)}(\bar{\nu}) \geq x_{j}$ by the convexity relation between $\ell^{(i)}-1$ and $j$ (if $j=\ell^{(i)}$ we can directly show the inequality without the second term). By the minimality of $\bar{s}$, we have $\bar{s} \leq x_{j}$. Therefore we obtain $\bar{s}=x_{j}$ which implies that the string $\left(j, x_{j}\right)$ is singular with length larger than $\ell^{(i)}-1$. However, we know that $\delta^{-1}$ will add a box to the length $\ell^{(i)}-1$ string of $(\bar{\nu}, \bar{J})^{(i)}$. This is a contradiction since $\ell^{(i+1)}=\infty$ implies that $\delta^{-1}$ will add a box to the longest possible string. Therefore we conclude that the longest string of $(\bar{\nu}, \bar{J})^{(i)}$ has length $\ell^{(i)}-1$. Since we have $P_{\ell^{(i)}-1}^{(i)}(\bar{\nu}) \geq P_{\ell^{(i)}}^{(i)}(\bar{\nu})$, the convexity relation between $\ell^{(i)}-1$ and $\infty$ gives $P_{\ell^{(i)}-1}^{(i)}(\bar{\nu}) \geq P_{\ell^{(i)}}^{(i)}(\bar{\nu}) \geq \cdots \geq P_{\infty}^{(i)}(\bar{\nu})$. However this is a contradiction since we already know that $P_{\ell^{(i)}}^{(i)}(\bar{\nu}) \leq \bar{s}$ and $P_{\infty}^{(i)}(\bar{\nu})>\bar{s}$ by $\varphi_{i}(\bar{\nu}, \bar{J})=P_{\infty}^{(i)}(\bar{\nu})-\bar{s}>0$. Thus we have shown that the relation $P_{\ell^{(i)}}^{(i)}(\bar{\nu})>\bar{s}$ is always satisfied. Hence we obtain $s \geq \bar{s}$.

By combining the two inequalities, we conclude that $s=\bar{s}$.

(2) During the proof of the previous lemma, we have shown that the only possible case under the assumption is $s=\bar{s}$.

Example 6.8. Consider the following rigged configuration $(\nu, J)$ of type $\left(B^{1,1}\right)^{\otimes 4} \otimes B^{1,3} \otimes B^{2,1} \otimes$ $B^{2,2} \otimes B^{3,1}$ of $D_{5}^{(1)}$
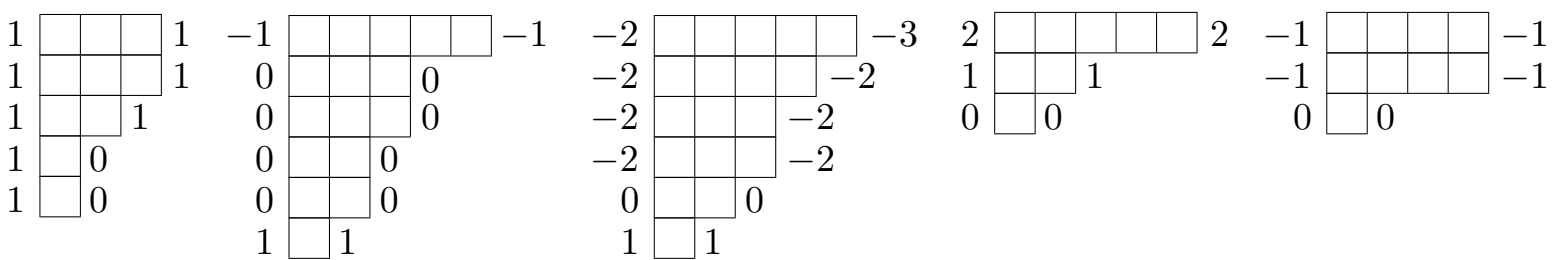

This is the example for Case (c-2) of the proof of Lemma 6.6. The corresponding image $\Phi^{-1}(\nu, J)$ is

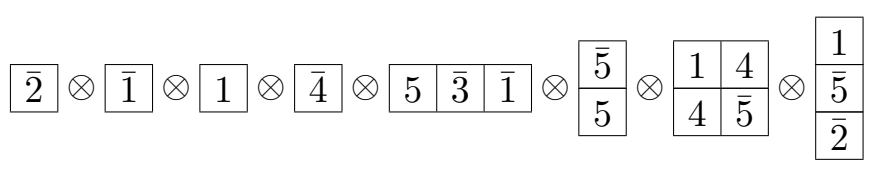

We have $\widetilde{f}_{2}(\nu, J)=0$ since we have $P_{6}^{(2)}(\widetilde{\nu})=-3 .(\bar{\nu}, \bar{J})$ is given as follows: 

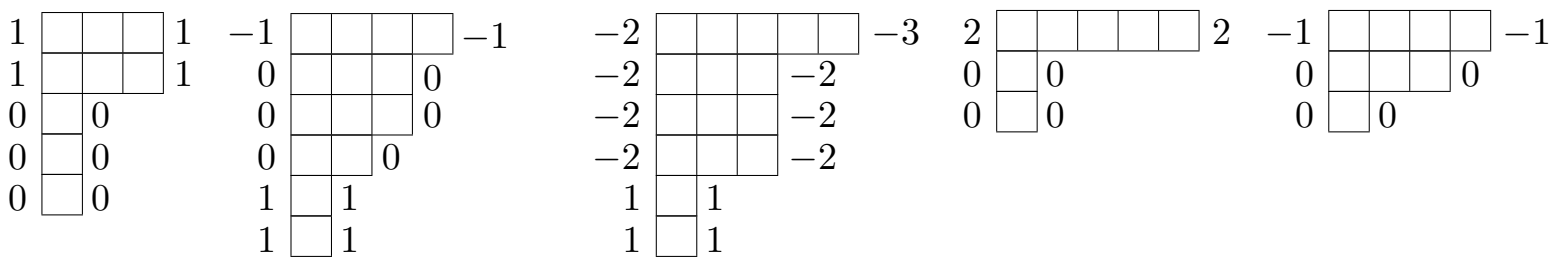

Note that the minimal rigging -1 of $(\nu, J)^{(2)}$ is preserved before and after $\delta$ in a nontrivial way. We have $\widetilde{f}_{2}(\bar{\nu}, \bar{J}) \neq 0$ since we have $P_{5}^{(2)}(\widetilde{\bar{\nu}})=-2$.

Proposition 6.9. Let us consider the rigged configuration $(\nu, J)$ of type $B^{1,1} \otimes \bar{B}$. Suppose that we have the commutativity of $\widetilde{f}_{i}$ and $\Phi$ for $\bar{B}$. Suppose that $\tilde{f}_{i}(\nu, J)=0$ and $\widetilde{f}_{i}(\bar{\nu}, \bar{J}) \neq 0$. Let $b=\Phi(\nu, J)$ and $b^{\prime}=\Phi(\bar{\nu}, \bar{J})$. Then we have $\widetilde{f}_{i}(b)=0, \widetilde{f}_{i}\left(b^{\prime}\right) \neq 0$ and $\Phi\left(\widetilde{f}_{i}(\nu, J)\right)=\widetilde{f}_{i}(\Phi(\nu, J))$.

Proof. According to Lemma 6.6, we see that there are only two possibilities. The first case is $\ell^{(i)}<\infty$ and $\ell^{(i+1)}=\infty$. In this case we have $b=(i+1) \otimes b^{\prime}$ and $P_{\infty}^{(i)}(\bar{\nu})=P_{\infty}^{(i)}(\nu)+1$. The second case is $\ell_{(i)}<\infty$ and $\ell_{(i-1)}=\infty$. In this case we have $\bar{i} \otimes b^{\prime}$ and $P_{\infty}^{(i)}(\bar{\nu})=P_{\infty}^{(i)}(\nu)+1$.

Recall that we have $\varphi_{i}\left(b^{\prime}\right)=\varphi_{i}(\bar{\nu}, \bar{J})>0$ by the induction hypothesis. Let us show that in fact we have $\varphi_{i}(\bar{\nu}, \bar{J})=1$. From Theorem 3.8, we compute

$$
\varphi_{i}(\bar{\nu}, \bar{J})=P_{\infty}^{(i)}(\bar{\nu})-\bar{s}=P_{\infty}^{(i)}(\nu)-s+1=1 .
$$

Here we have used the relation $s=\bar{s}$ from Lemma 6.7 and the assumption $\varphi(\nu, J)=P_{\infty}^{(i)}(\nu)-s=0$. Thus $\varphi_{i}(\bar{\nu}, \bar{J})=1$.

Then by the induction hypothesis we have $\varphi_{i}\left(b^{\prime}\right)=\varphi_{i}(\bar{\nu}, \bar{J})=1$. On the other hand, recall that we have $\varepsilon_{i}(i+1)=\varepsilon_{i}(\bar{i})=1$. Then from the tensor product rule at Section 2.1, we conclude that $\widetilde{f}_{i}(b)=0$. Hence we have $\Phi\left(\widetilde{f}_{i}(\nu, J)\right)=\widetilde{f}_{i}(\Phi(\nu, J))$.

\subsection{Proof for (4)}

Proposition 6.10. Let $b \in B^{1,1} \otimes \bar{B}$ and suppose that we have shown the commutativity of $\widetilde{f}_{i}$ and $\Phi$ for the elements of $\bar{B}$. Suppose that we have $\widetilde{f}_{i}(b)=0$ and $\widetilde{f}_{i}\left(b^{\prime}\right) \neq 0$ where $b^{\prime} \in \bar{B}$ is the corresponding part of $b$. Then we have $\widetilde{f}_{i}(\nu, J)=0, \widetilde{f}_{i}(\bar{\nu}, \bar{J}) \neq 0$ and $\Phi^{-1}\left(\widetilde{f}_{i}(b)\right)=\widetilde{f}_{i}\left(\Phi^{-1}(b)\right)$.

Proof. By the assumption, we have $b=(i+1) \otimes b^{\prime}$ or $b=\bar{i} \otimes b^{\prime}$ and $\varphi_{i}\left(b^{\prime}\right)=1$. In the first case we have $\ell^{(i)}<\infty$ and $\ell^{(i+1)}=\infty$ and in the second case we have $\ell_{(i)}<\infty$ and $\ell_{(i-1)}=\infty$. In both cases we have $P_{\infty}^{(i)}(\nu)=P_{\infty}^{(i)}(\bar{\nu})-1$. From the condition $\varphi_{i}\left(b^{\prime}\right)=1$ together with the induction hypothesis we have $\varphi_{i}\left(b^{\prime}\right)=\varphi_{i}(\bar{\nu}, \bar{J})=1$, which implies $P_{\infty}^{(i)}(\bar{\nu})=\bar{s}+1$ by Theorem 3.8. Here $\bar{s}$ is the minimal rigging of $(\bar{\nu}, \bar{J})^{(i)}$. Then we have

$$
\varphi_{i}(\nu, J)=P_{\infty}^{(i)}(\nu)-s=P_{\infty}^{(i)}(\bar{\nu})-1-s=\bar{s}-s .
$$

If we show $s \geq \bar{s}$ we have $\varphi_{i}(\nu, J)=0$ as desired. Note that this result leads to $\Phi^{-1}\left(\widetilde{f}_{i}(b)\right)=$ $\widetilde{f}_{i}\left(\Phi^{-1}(b)\right)$ also.

Let us show $s \geq \bar{s}$. If $\ell^{(i)}<\infty$ and $\ell^{(i+1)}=\infty$ we can use the same arguments of Step 2 of the proof of Lemma 6.7(1) since we do not use the assumption $\varphi_{i}(\nu, J)=0$ there. If $\ell_{(i)}<\infty$ and $\ell_{(i-1)}=\infty$, again we can use the same arguments of Step 2 of the proof of Lemma 6.7(1) by replacing $\ell^{(i)}$ and $\ell^{(i+1)}$ there by $\ell_{(i)}$ and $\ell_{(i-1)}$ respectively.

\section{Proof of Proposition 4.8: $\widetilde{e}_{i}$ version}

In this section we use a parallel notation that are introduced in Section 4.4 except for changing the role of $\widetilde{f}_{i}$ there by $\widetilde{e}_{i}$. For example, $\widetilde{e}_{i}$ acts on the string $\left(\ell, x_{\ell}\right)$ of $(\nu, J)^{(i)}$. 


\subsection{Proof for (5)}

Proposition 7.1. Let us consider the rigged configuration $(\nu, J)$ of type $B^{1,1} \otimes \bar{B}$. Suppose that we have the commutativity of $\widetilde{e}_{i}$ and $\widetilde{f}_{i}$ with $\Phi$ for $\bar{B}$. Suppose that $\widetilde{e}_{i}(\nu, J) \neq 0$ and $\widetilde{e}_{i}(\bar{\nu}, \bar{J})=0$. Let $b=\Phi(\nu, J)$ and $b^{\prime}=\Phi(\bar{\nu}, \bar{J})$. Then we have one of the following two cases:

(1) $b=(i+1) \otimes b^{\prime}, \widetilde{e}_{i}(b)$ is defined, $\widetilde{e}_{i}\left(b^{\prime}\right)$ is undefined and $\Phi\left(\widetilde{e}_{i}(\nu, J)\right)=i \otimes b^{\prime}$,

(2) $b=\bar{i} \otimes b^{\prime}, \widetilde{e}_{i}(b)$ is defined, $\widetilde{e}_{i}\left(b^{\prime}\right)$ is undefined and $\Phi\left(\widetilde{e}_{i}(\nu, J)\right)=\overline{i+1} \otimes b^{\prime}$.

For the proof, we show the following properties.

Proposition 7.2. $\widetilde{e}_{i}(\nu, J)$ is defined and $\widetilde{e}_{i}(\bar{\nu}, \bar{J})$ is undefined if and only if one of the following conditions is satisfied:

(1) $\ell^{(i)}<\infty, \ell^{(i+1)}=\infty$ and all the riggings of $(\nu, J)^{(i)}$ are non-negative except for the longest string whose rigging is -1 . Moreover we have

$$
P_{\ell^{(i)}-1}^{(i)}(\bar{\nu})=P_{\ell^{(i)}}^{(i)}(\bar{\nu})=\cdots=P_{\infty}^{(i)}(\bar{\nu})=0 .
$$

(2) $\ell_{(i)}<\infty, \ell_{(i-1)}=\infty$ and all the riggings of $(\nu, J)^{(i)}$ are non-negative except for the longest string $(s)$ whose rigging is -1 . Moreover we have

$$
P_{\ell_{(i)}-1}^{(i)}(\bar{\nu})=P_{\ell_{(i)}}^{(i)}(\bar{\nu})=\cdots=P_{\infty}^{(i)}(\bar{\nu})=0 .
$$

Proof. By the assumptions $\widetilde{e}_{i}(\nu, J) \neq 0$ and $\widetilde{e}_{i}(\bar{\nu}, \bar{J})=0$, we see that $\delta$ changes $(\nu, J)^{(i)}$. Thus we have $\ell^{(i)}<\infty$. Also, from $\widetilde{e}_{i}(\bar{\nu}, \bar{J})=0$, we see that all the riggings of $(\bar{\nu}, \bar{J})^{(i)}$ are non-negative.

(1) Suppose that we have $\ell^{(i+1)}=\infty$. Suppose if possible that we have $m_{j}^{(i)}(\bar{\nu})>0$ for some $\ell^{(i)} \leq j$. Let us choose the minimal such $j$. Note that by the condition $\ell^{(i)} \leq j$, the string $\left(j, x_{j}\right)$ is different from the string $\left(\ell^{(i)}-1, P_{\ell^{(i)}-1}^{(i)}(\bar{\nu})\right)$ created by $\delta$. By the definition of $\delta^{-1}$, $\ell^{(i)}<\infty$ and $\ell^{(i+1)}=\infty$ imply that the string $\left(\ell^{(i)}-1, P_{\ell^{(i)}-1}^{(i)}(\bar{\nu})\right)$ is the longest singular string of $(\bar{\nu}, \bar{J})^{(i)}$. In particular, the string $\left(j, x_{j}\right)$ is not singular so that we have $P_{j}^{(i)}(\bar{\nu})>x_{j} \geq 0$. On the other hand, we have $P_{\ell^{(i)}-1}^{(i)}(\bar{\nu}) \geq 0$ since $\widetilde{e}_{i}(\bar{\nu}, \bar{J})=0$. Let us show $P_{\ell^{(i)}}^{(i)}(\bar{\nu})>0$. If $\ell^{(i)}=j$ it is already proved. Thus suppose that $\ell^{(i)}<j$. Since we have $m_{k}^{(i)}(\bar{\nu})=0$ for all $\ell^{(i)}<k<j$, the only possibility that is compatible with $P_{\ell^{(i)}-1}^{(i)}(\bar{\nu}) \geq 0, P_{j}^{(i)}(\bar{\nu})>0$ and the convexity relation of $P_{k}^{(i)}(\bar{\nu})$ for $\ell^{(i)}-1 \leq k \leq j$ is $P_{\ell^{(i)}}^{(i)}(\bar{\nu})>0$. In conclusion, we have $P_{\ell^{(i)}}^{(i)}(\nu) \geq 0$ by $P_{\ell^{(i)}}^{(i)}(\nu)=P_{\ell^{(i)}}^{(i)}(\bar{\nu})-1$ which follows from the relations $\ell^{(i)}<\infty$ and $\ell^{(i+1)}=\infty$. Since all the riggings of $(\bar{\nu}, \bar{J})^{(i)}$ are non-negative, the relation $P_{\ell^{(i)}}^{(i)}(\nu) \geq 0$ implies that all the riggings of $(\nu, J)^{(i)}$ are non-negative. This contradicts the assumption $\widetilde{e}_{i}(\nu, J) \neq 0$. Hence the string $\left(\ell^{(i)}-1, P_{\ell^{(i)}-1}^{(i)}(\bar{\nu})\right)$ is the longest string of $(\bar{\nu}, \bar{J})^{(i)}$.

Let us show that $P_{\ell^{(i)}}^{(i)}(\bar{\nu})=0$. Suppose if possible that we have $P_{\ell^{(i)}}^{(i)}(\bar{\nu})>0$. Then we have $P_{\ell^{(i)}}^{(i)}(\nu) \geq 0$ by $P_{\ell^{(i)}}^{(i)}(\nu)=P_{\ell^{(i)}}^{(i)}(\bar{\nu})-1$. This contradicts the assumption $\widetilde{e}_{i}(\nu, J) \neq 0$. Therefore we have $P_{\ell^{(i)}}^{(i)}(\bar{\nu})=0$. In particular, the string $\left(\ell^{(i)}, P_{\ell^{(i)}}^{(i)}(\nu)\right)=\left(\ell^{(i)},-1\right)$ is the only string of $(\nu, J)^{(i)}$ which has the negative rigging.

Finally let us show that $P_{\ell^{(i)}-1}^{(i)}(\bar{\nu})=\cdots=P_{\infty}^{(i)}(\bar{\nu})=0$. By the convexity relation of $P_{k}^{(i)}(\bar{\nu})$ between $\ell^{(i)}-1 \leq k<\infty$ and the condition $P_{\infty}^{(i)}(\bar{\nu})>-\infty$, we have $P_{\ell^{(i)}-1}^{(i)}(\bar{\nu}) \leq P_{\ell^{(i)}}^{(i)}(\bar{\nu}) \leq \cdots \leq$ 
$P_{\infty}^{(i)}(\bar{\nu})$. Recall that we have $P_{\ell^{(i)}-1}^{(i)}(\bar{\nu}) \geq 0$ by $\widetilde{e}_{i}(\bar{\nu}, \bar{J})=0$ and $P_{\ell^{(i)}}^{(i)}(\bar{\nu})=0$ by the previous paragraph. Then the only possibility is $P_{\ell^{(i)}-1}^{(i)}(\bar{\nu})=P_{\ell^{(i)}}^{(i)}(\bar{\nu})=\cdots=P_{\infty}^{(i)}(\bar{\nu})=0$.

(2) In this case we assume that $\ell^{(i+1)}<\infty$.

Step 1. We shall show that $\ell_{(i+1)}<\infty$. Suppose if possible that $\ell_{(i+1)}=\infty$. Consider the case $\ell^{(i)}=\ell^{(i+1)}$. Then we have $P_{\ell^{(i)}}^{(i)}(\nu)=P_{\ell^{(i)}}^{(i)}(\bar{\nu})$. This implies that all the riggings of $(\nu, J)^{(i)}$ are non-negative. This contradicts the assumption $\widetilde{e}_{i}(\nu, J) \neq 0$.

Thus we are left with the case $\ell^{(i)}<\ell^{(i+1)}$. Then we have $P_{\ell^{(i)}}^{(i)}(\nu)=P_{\ell^{(i)}}^{(i)}(\bar{\nu})-1$. In order to have $\widetilde{e}_{i}(\nu, J) \neq 0$, we must have $P_{\ell^{(i)}}^{(i)}(\bar{\nu})=0$. Suppose if possible that there are strings of $(\bar{\nu}, \bar{J})^{(i)}$ which are longer than $\ell^{(i)}-1$. Let $j$ be the minimal integer such that $m_{j}^{(i)}(\bar{\nu})>0$ and $\ell^{(i)} \leq j$. Then the string $\left(j, x_{j}\right)$ of $(\bar{\nu}, \bar{J})^{(i)}$ must satisfy $P_{j}^{(i)}(\bar{\nu}) \geq x_{j} \geq 0$. Similarly, the string $\left(\ell^{(i)}-1, P_{\ell^{(i)}-1}^{(i)}(\bar{\nu})\right)$ must satisfy $P_{\ell^{(i)}-1}^{(i)}(\bar{\nu}) \geq 0$. Then the only possibility that is compatible with the convexity relation of $P_{k}^{(i)}(\bar{\nu})$ between $\ell^{(i)}-1 \leq k \leq j$ is the relation

$$
P_{\ell^{(i)}-1}^{(i)}(\bar{\nu})=P_{\ell^{(i)}}^{(i)}(\bar{\nu})=\cdots=P_{j}^{(i)}(\bar{\nu})=0 .
$$

From $(7.1)$ we have $m_{k}^{(i+1)}(\bar{\nu})=0$ for all $\ell^{(i)} \leq k<j$. Recall that by definition of $\ell^{(i+1)}$ there is the string $\left(\ell^{(i+1)}-1, P_{\ell^{(i+1)}-1}^{(i+1)}(\bar{\nu})\right)$ of $(\bar{\nu}, \bar{J})^{(i+1)}$. Then we see that its length satisfies $j \leq \ell^{(i+1)}-1$ by $\ell^{(i)} \leq \ell^{(i+1)}-1$. Also from $(7.1)$ and the assumption $\widetilde{e}_{i}(\bar{\nu}, \bar{J})=0$, we see that the string $\left(j, x_{j}\right)$ is singular since we have $0=P_{j}^{(i)}(\bar{\nu}) \geq x_{j} \geq 0$. By the relation $j \leq \ell^{(i+1)}-1, \delta^{-1}$ will add a box to the string $\left(j, x_{j}\right)$ of $(\bar{\nu}, \bar{J})^{(i)}$ instead of the string $\left(\ell^{(i)}-1, P_{\ell^{(i)}-1}^{(i)}(\bar{\nu})\right)$. This is a contradiction since we have $\ell^{(i)}-1<j$. In conclusion we see that the string $\left(\ell^{(i)}-1, P_{\ell^{(i)}-1}^{(i)}(\bar{\nu})\right)$ is the longest string of $(\bar{\nu}, \bar{J})^{(i)}$.

We know that $P_{\ell^{(i)}}^{(i)}(\bar{\nu})=0$. Since $\widetilde{e}_{i}(\bar{\nu}, \bar{J})=0$, we have $P_{\ell^{(i)}-1}^{(i)}(\bar{\nu}) \geq 0$ and, in particular, $P_{\ell^{(i)}-1}^{(i)}(\bar{\nu}) \geq P_{\ell^{(i)}}^{(i)}(\bar{\nu})$. Since there is no string of $(\bar{\nu}, \bar{J})^{(i)}$ which is longer than $\ell^{(i)}-1$, we can use the convexity relation of $P_{k}^{(i)}(\bar{\nu})$ between $\ell^{(i)}-1 \leq k<\infty$ with $P_{\infty}^{(i)}(\bar{\nu})>-\infty$ to conclude that $P_{\ell^{(i)}-1}^{(i)}(\bar{\nu}) \leq P_{\ell^{(i)}}^{(i)}(\bar{\nu}) \leq \cdots \leq P_{\infty}^{(i)}(\bar{\nu})$. By the relation $P_{\ell^{(i)}-1}^{(i)}(\bar{\nu}) \geq P_{\ell^{(i)}}^{(i)}(\bar{\nu})$ we conclude that $P_{\ell^{(i)}-1}^{(i)}(\bar{\nu})=P_{\ell^{(i)}}^{(i)}(\bar{\nu})=\cdots=P_{\infty}^{(i)}(\bar{\nu})$ is the only possibility that is compatible with the convexity relation. Then we have $m_{k}^{(i+1)}(\bar{\nu})=0$ for all $\ell^{(i)} \leq k$. This is a contradiction since we are assuming that $\ell^{(i)}<\ell^{(i+1)}$. Hence we have $\ell_{(i+1)}<\infty$.

Step 2. We shall show that $\ell_{(i)}<\infty$. Suppose if possible that $\ell_{(i)}=\infty$. In order to have $\widetilde{e}_{i}(\nu, J) \neq 0$, we must have $P_{\ell^{(i)}}^{(i)}(\nu)=P_{\ell^{(i)}}^{(i)}(\bar{\nu})-1$. Under the condition $\ell_{(i)}=\infty$, this is only possible if we have $\ell^{(i)}<\ell^{(i+1)}$. Then we can use the same argument of the corresponding part of Step 1 to deduce a contradiction. Thus we have $\ell_{(i)}<\infty$.

Step 3. We shall show that $\ell_{(i-1)}=\infty$. Suppose if possible that $\ell_{(i-1)}<\infty$. Then we can use the same arguments of Step 1 if we replace $\ell^{(i)}$ and $\ell^{(i+1)}$ there by $\ell_{(i)}$ and $\ell_{(i-1)}$ respectively to deduce a contradiction. Thus we have $\ell_{(i-1)}=\infty$.

Step 4. We shall show that the length $\ell^{(i)}$ string of $(\nu, J)^{(i)}$ created by $\delta^{-1}$ has non-negative rigging if $\ell^{(i)}<\ell_{(i)}$. For this we have to show that $P_{\ell^{(i)}}^{(i)}(\nu) \geq 0$. Recall that we have $P_{\ell^{(i)}-1}^{(i)}(\bar{\nu}) \geq 0$ by the assumption $\widetilde{e}_{i}(\bar{\nu}, \bar{J})=0$. On the other hand, let $j$ be the smallest integer such that $m_{j}^{(i)}(\bar{\nu})>0$ and $\ell^{(i)} \leq j$. By $\ell^{(i)}<\ell_{(i)}$ we have $j \leq \ell_{(i)}$. Then by the assumption $\widetilde{e}_{i}(\bar{\nu}, \bar{J})=0$ we have $P_{j}^{(i)}(\bar{\nu}) \geq 0$.

If $\ell^{(i)}=\ell^{(i+1)}$, we have $P_{\ell^{(i)}}^{(i)}(\nu) \geq P_{\ell^{(i)}}^{(i)}(\bar{\nu})$ by $\ell^{(i)}<\ell_{(i)}$. Then by the convexity relation of $P_{k}^{(i)}(\bar{\nu})$ between $\ell^{(i)}-1 \leq k \leq j$ we have $P_{\ell^{(i)}}^{(i)}(\nu) \geq P_{\ell^{(i)}}^{(i)}(\bar{\nu}) \geq \min \left\{P_{\ell^{(i)}-1}^{(i)}(\bar{\nu}), P_{j}^{(i)}(\bar{\nu})\right\} \geq 0$. 
Next let us consider the case $\ell^{(i)}<\ell^{(i+1)}$. Then we have $P_{\ell^{(i)}}^{(i)}(\nu)=P_{\ell^{(i)}}^{(i)}(\bar{\nu})-1$. Then one can attain $P_{\ell^{(i)}}^{(i)}(\nu)=-1$ only if $P_{\ell^{(i)}-1}^{(i)}(\bar{\nu})=\cdots=P_{j}^{(i)}(\bar{\nu})=0$. This relation implies that $m_{k}^{(i+1)}(\bar{\nu})=0$ for all $\ell^{(i)}-1<k<j$. Then we have $j \leq \ell^{(i+1)}-1$ since we have $\ell^{(i)}-1<\ell^{(i+1)}-1$ by the assumption. Let us consider the string $\left(j, x_{j}\right)$ of $(\bar{\nu}, \bar{J})^{(i)}$. Then we have $P_{j}^{(i)}(\bar{\nu}) \geq x_{j} \geq 0$ by the assumption $\widetilde{e}_{i}(\bar{\nu}, \bar{J})=0$. Since $P_{j}^{(i)}(\bar{\nu})=0$ we see that the string $\left(j, x_{j}\right)$ is singular and its length satisfies $\ell^{(i)}-1<j \leq \ell^{(i+1)}-1$. Thus $\delta^{-1}$ will add a box to the string $\left(j, x_{j}\right)$ instead of the length $\ell^{(i)}-1$ string. This is a contradiction. To summarize, we have shown that the length $\ell^{(i)}$ string of $(\nu, J)^{(i)}$ created by $\delta^{-1}$ has non-negative rigging if $\ell^{(i)}<\ell_{(i)}$.

Then we can use a parallel arguments of the previous case (1) to deduce that the string $\left(\ell_{(i)}-1, P_{\ell_{(i)}-1}^{(i)}(\bar{\nu})\right)$ is the longest string of $(\bar{\nu}, \bar{J})^{(i)}$.

Step 5. Finally let us show that $P_{\ell_{(i)}-1}^{(i)}(\bar{\nu})=\cdots=P_{\infty}^{(i)}(\bar{\nu})=0$. Since the length $\ell_{(i)}-1$ string is the longest string of $(\bar{\nu}, \bar{J})^{(i)}$, we have $P_{\ell_{(i)}-1}^{(i)}(\bar{\nu}) \leq P_{\ell_{(i)}}^{(i)}(\bar{\nu}) \leq \cdots \leq P_{\infty}^{(i)}(\bar{\nu})$ by the convexity relation of $P_{k}^{(i)}(\bar{\nu})$ between $\ell_{(i)}-1 \leq k<\infty$ and $P_{\infty}^{(i)}(\bar{\nu})>-\infty$. Since we have $P_{\ell_{(i)}}^{(i)}(\nu)=-1$, we have $P_{\ell_{(i)}}^{(i)}(\bar{\nu})=0$ by $P_{\ell_{(i)}}^{(i)}(\nu)=P_{\ell_{(i)}}^{(i)}(\bar{\nu})-1$. Since we have $P_{\ell_{(i)}-1}^{(i)}(\bar{\nu}) \geq 0$ by $\widetilde{e}_{i}(\bar{\nu}, \bar{J})=0$, we conclude that $P_{\ell_{(i)}-1}^{(i)}(\bar{\nu})=P_{\ell_{(i)}}^{(i)}(\bar{\nu})=\cdots=P_{\infty}^{(i)}(\bar{\nu})=0$.

Proof of Proposition 7.1. We divide the proof into two cases following Proposition 7.2.

(1) Let us consider the case $\ell^{(i)}<\infty$ and $\ell^{(i+1)}=\infty$. In this case we have $b=(i+1) \otimes b^{\prime}$. By the assumption we have $\varepsilon_{i}\left(b^{\prime}\right)=\varepsilon_{i}(\bar{\nu}, \bar{J})=0$. From Proposition 7.2 we know that $P_{\infty}^{(i)}(\bar{\nu})=0$ and the minimal rigging of $(\bar{\nu}, \bar{J})^{(i)}$ is 0 . Therefore we have $\varphi_{i}(\bar{\nu}, \bar{J})=0$ by Theorem 3.8. Then by the assumption we have $\varphi_{i}\left(b^{\prime}\right)=\varphi_{i}(\bar{\nu}, \bar{J})=0$. Thus $\varepsilon_{i}(b)=1$, that is, $\widetilde{e}_{i}(b) \neq 0$.

Finally let us show $\Phi\left(\widetilde{e}_{i}(\nu, J)\right)=i \otimes b^{\prime}$. Recall that the longest string of $(\bar{\nu}, \bar{J})^{(i)}$ has length $\ell^{(i)}-1$. Therefore we have $m_{\ell^{(i)}}^{(i)}(\nu)=1$. Thus if $\ell^{(i-1)}=\ell^{(i)}$ we see that we cannot remove a box from $\left(\widetilde{e}_{i}(\nu, J)\right)^{(i)}$ by $\delta$ since the length of the longest string of $\left(\widetilde{e}_{i}(\nu, J)\right)^{(i)}$ is $\ell^{(i)}-1$ and thus all the strings of $\left(\widetilde{e}_{i}(\nu, J)\right)^{(i)}$ are strictly shorter than $\ell^{(i-1)}$. In this case we have $\Phi\left(\widetilde{e}_{i}(\nu, J)\right)=i \otimes b^{\prime}$. Therefore let us suppose that $\ell^{(i-1)}<\ell^{(i)}$ in the following. Recall that we have $P_{\ell^{(i)}-1}^{(i)}(\bar{\nu})=0$. Then we have $P_{\ell^{(i)}-1}^{(i)}(\nu)=1$ by $\ell^{(i-1)}<\ell^{(i)}$. We know that $\widetilde{e}_{i}$ changes the string $\left(\ell^{(i)},-1\right)$ of $(\nu, J)^{(i)}$ into $\left(\ell^{(i)}-1,0\right)$. In particular, the latter string is non-singular by $P_{\ell^{(i)}-1}^{(i)}(\widetilde{\nu})=P_{\ell^{(i)}-1}^{(i)}(\nu)=1$. Thus $\widetilde{\ell}^{(i)}=\infty$ also. In conclusion we have $\Phi\left(\widetilde{e}_{i}(\nu, J)\right)=i \otimes b^{\prime}$.

(2) The proof for this case is almost identical to the previous case if we replace $\ell^{(i-1)}$ and $\ell^{(i)}$ by $\ell_{(i)}$ and $\ell_{(i+1)}$ respectively.

Example 7.3. Consider the following rigged configuration $(\nu, J)$ of type $\left(B^{1,1}\right)^{\otimes 4} \otimes B^{1,3} \otimes B^{2,1} \otimes$ $B^{2,2} \otimes B^{3,1}$ of $D_{5}^{(1)}$

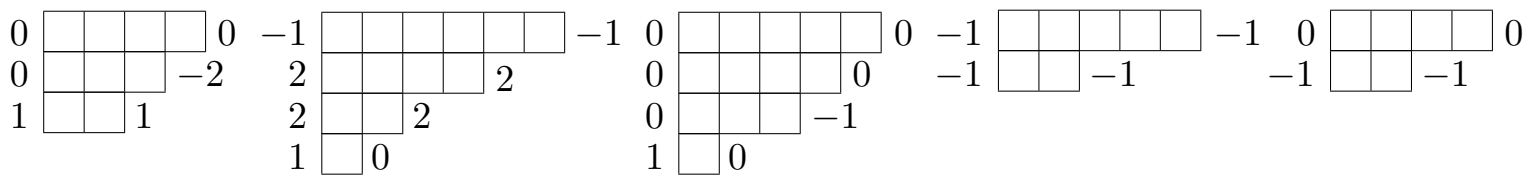

The corresponding image $\Phi^{-1}(\nu, J)$ is

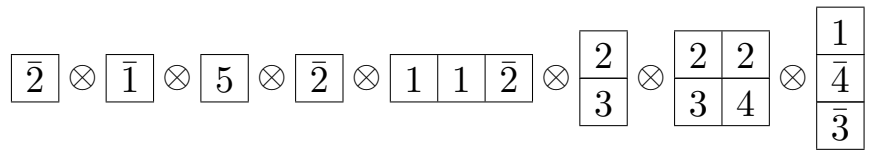

We have $\widetilde{e}_{2}(\nu, J) \neq 0$ since $(\nu, J)^{(2)}$ has a negative rigging. Here $\widetilde{e}_{2}$ changes the string $(6,-1)$ of $(\nu, J)^{(2)}$ into $(5,0)$. Since $P_{5}^{(i)}(\widetilde{\nu})=1$, the latter string is non-singular. Thus the leftmost letter 
of $\Phi(\widetilde{\nu}, \widetilde{J})$ is $\overline{3}$ and the rest part is the same as the corresponding part of the above path. $(\bar{\nu}, \bar{J})$ is given as follows:
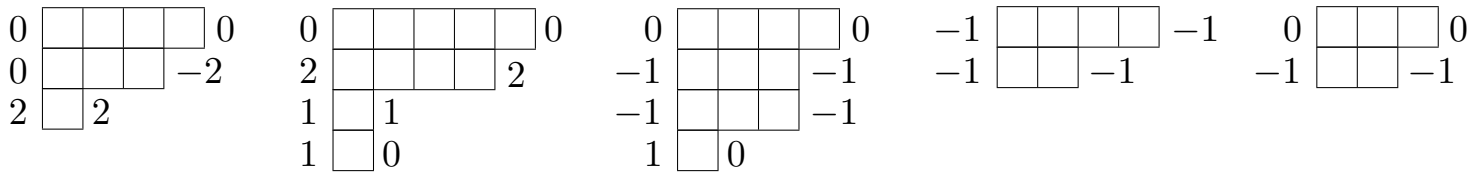

Since all the riggings of $(\bar{\nu}, \bar{J})^{(2)}$ are non-negative, we have $\widetilde{e}_{2}(\bar{\nu}, \bar{J})=0$.

\subsection{Proof for $(6)$}

Proposition 7.4. Let $b \in B^{1,1} \otimes \bar{B}$ and suppose that we have shown the commutativity of $\widetilde{e}_{i}$ and $\widetilde{f}_{i}$ with $\Phi$ for the elements of $\bar{B}$. Suppose that $\widetilde{e}_{i}(b) \neq 0$ and $\widetilde{e}_{i}\left(b^{\prime}\right)=0$ where $b^{\prime} \in \bar{B}$ is the corresponding part of $b$. Then $\widetilde{e}_{i}(\nu, J) \neq 0, \widetilde{e}_{i}(\bar{\nu}, \bar{J})=0$ and $\Phi^{-1}\left(\widetilde{e}_{i}(b)\right)=\widetilde{e}_{i}\left(\Phi^{-1}(b)\right)$.

Proof. By the assumption, we have the following two possibilities:

(a) $b=(i+1) \otimes b^{\prime}$ and $\widetilde{e}_{i}(b)=i \otimes b^{\prime}$,

(b) $b=\bar{i} \otimes b^{\prime}$ and $\widetilde{e}_{i}(b)=\overline{i+1} \otimes b^{\prime}$.

In both cases we have $\varphi_{i}\left(b^{\prime}\right)=0$. Note that we have $\widetilde{e}_{i}(\bar{\nu}, \bar{J})=0$ by the assumption.

Case (a). By the assumption we have $\varepsilon_{i}(\bar{\nu}, \bar{J})=0$ and $\varphi_{i}(\bar{\nu}, \bar{J})=0$. Then from Theorem 3.8 we have $P_{\infty}^{(i)}(\bar{\nu})=\varphi_{i}(\bar{\nu}, \bar{J})-\varepsilon_{i}(\bar{\nu}, \bar{J})=0$. Let $l:=\bar{\nu}_{1}^{(i)}$ be the length of the longest string of $(\bar{\nu}, \bar{J})^{(i)}$. Then we can use the convexity relation of $P_{k}^{(i)}(\bar{\nu})$ between $l \leq k \leq \infty$ to deduce that $P_{l}^{(i)}(\bar{\nu})=P_{l+1}^{(i)}(\bar{\nu})=\cdots=P_{\infty}^{(i)}(\bar{\nu})=0$. Consider the string $\left(l, x_{l}\right)$ of $(\bar{\nu}, \bar{J})^{(i)}$. Then from $\widetilde{e}_{i}(\bar{\nu}, \bar{J})=0$ we have $P_{l}^{(i)}(\bar{\nu}) \geq x_{l} \geq 0$. Thus we have $x_{l}=0$ and, in particular, the string $\left(l, x_{l}\right)=(l, 0)$ is singular. Since we have $b=(i+1) \otimes b^{\prime}$, we see that $\delta^{-1}$ adds a box to the string $(l, 0)$ of $(\bar{\nu}, \bar{J})^{(i)}$, that is, $l+1=\ell^{(i)}$. Since $\ell^{(i+1)}=\infty$, we see that $P_{\ell^{(i)}}^{(i)}(\nu)=P_{\ell^{(i)}}^{(i)}(\bar{\nu})-1=-1$. To summarize, the longest string of $(\nu, J)^{(i)}$ is $\left(\ell^{(i)},-1\right)$ and therefore we have $\widetilde{e}_{i}(\nu, J) \neq 0$.

Since $\delta^{-1}$ does not change the riggings of the strings except for the longest one, we see that the unique string of $(\nu, J)^{(i)}$ which has negative rigging is $\left(\ell^{(i)},-1\right)$. Thus $\widetilde{e}_{i}$ acts on the string $\left(\ell^{(i)},-1\right)$ and changes it to $\left(\ell^{(i)}-1,0\right)$. If $\ell^{(i-1)}=\ell^{(i)}$ we see that $\delta$ cannot remove a box from $(\widetilde{\nu}, \widetilde{J})^{(i)}$ since all the strings are strictly shorter than $\ell^{(i-1)}$. Thus $\Phi^{-1}\left(\widetilde{e}_{i}(b)\right)=i \otimes b^{\prime}$. So suppose that $\ell^{(i-1)}<\ell^{(i)}$. Then we have $P_{\ell^{(i)}-1}^{(i)}(\widetilde{\nu})=P_{\ell^{(i)}-1}^{(i)}(\nu)=P_{\ell^{(i)}-1}^{(i)}(\bar{\nu})+1=1$. Thus the string $\left(\ell^{(i)}-1,0\right)$ created by $\widetilde{e}_{i}$ is non-singular. Therefore we have $\widetilde{\ell}^{(i)}=\infty$ and hence $\Phi^{-1}\left(\widetilde{e}_{i}(b)\right)=i \otimes b^{\prime}$.

Case (b). Proof of this case is almost identical to the previous Case (a).

\subsection{Proof for $(7)$}

Proposition 7.5. The situation $\widetilde{e}_{i}(\nu, J)=0$ and $\widetilde{e}_{i}(\bar{\nu}, \bar{J}) \neq 0$ cannot happen.

Proof. Suppose if possible that we have $\widetilde{e}_{i}(\nu, J)=0$ and $\widetilde{e}_{i}(\bar{\nu}, \bar{J}) \neq 0$. Then $\delta$ must change some strings of $(\nu, J)^{(i)}$. Thus we have $\ell^{(i)}<\infty$.

Step 1. Let us consider the case $\ell_{(i)}=\infty$. Then the string $\left(\ell^{(i)}-1, P_{\ell^{(i)}-1}^{(i)}(\bar{\nu})\right)$ is the unique string of $(\bar{\nu}, \bar{J})^{(i)}$ which has a negative rigging. In particular, we have $P_{\ell^{(i)}-1}^{(i)}(\bar{\nu})<0$. Let $j$ be the largest integer such that $m_{j}^{(i)}(\bar{\nu})>0$ and $j<\ell^{(i)}-1$. If there is no such string, set $j=0$. Then we must have $P_{j}^{(i)}(\bar{\nu}) \geq 0$. From the convexity relation of $P_{k}^{(i)}(\bar{\nu})$ between $j \leq k \leq \ell^{(i)}-1$, we see that $P_{\ell^{(i)}-2}^{(i)}(\bar{\nu})>P_{\ell^{(i)}-1}^{(i)}(\bar{\nu})$. 
On the other hand, from $\widetilde{e}_{i}(\nu, J)=0$ the string $\left(\ell^{(i)}, P_{\ell^{(i)}}^{(i)}(\nu)\right)$ must have a non-negative rigging so that we have $P_{\ell^{(i)}}^{(i)}(\nu) \geq 0$.

1. Let us consider the case $\ell^{(i)}<\ell^{(i+1)}$. Then we have $P_{\ell^{(i)}}^{(i)}(\nu)=P_{\ell^{(i)}}^{(i)}(\bar{\nu})-1$. Therefore we have $P_{\ell^{(i)}}^{(i)}(\bar{\nu}) \geq 1$. Thus

$$
-P_{\ell^{(i)}-2}^{(i)}(\bar{\nu})+2 P_{\ell^{(i)}-1}^{(i)}(\bar{\nu})-P_{\ell^{(i)}}^{(i)}(\bar{\nu}) \leq-3 .
$$

On the other hand, since we cannot have more than one strings of $(\bar{\nu}, \bar{J})^{(i)}$ which have negative riggings, we have $m_{\ell^{(i)}-1}^{(i)}(\bar{\nu})=1$. Thus

$$
m_{\ell^{(i)}-1}^{(i-1)}(\bar{\nu})-2 m_{\ell^{(i)}-1}^{(i)}(\bar{\nu})+m_{\ell^{(i)}-1}^{(i+1)}(\bar{\nu}) \geq-2 .
$$

This contradicts the convexity relation of Lemma 3.13.

2. Next, let us consider the case $\ell^{(i)}=\ell^{(i+1)}<\ell_{(i+1)}$. Then we have $P_{\ell^{(i)}}^{(i)}(\nu)=P_{\ell^{(i)}}^{(i)}(\bar{\nu})$ so that $P_{\ell^{(i)}}^{(i)}(\bar{\nu}) \geq 0$. Thus

$$
-P_{\ell^{(i)}-2}^{(i)}(\bar{\nu})+2 P_{\ell^{(i)}-1}^{(i)}(\bar{\nu})-P_{\ell^{(i)}}^{(i)}(\bar{\nu}) \leq-2 .
$$

On the other hand, we have $m_{\ell^{(i)}-1}^{(i)}(\bar{\nu})=1$ and $m_{\ell^{(i)}-1}^{(i+1)}(\bar{\nu}) \geq 1$. Thus

$$
m_{\ell^{(i)}-1}^{(i-1)}(\bar{\nu})-2 m_{\ell^{(i)}-1}^{(i)}(\bar{\nu})+m_{\ell^{(i)}-1}^{(i+1)}(\bar{\nu}) \geq-1 .
$$

Again this contradicts the convexity relation of Lemma 3.13.

3. Finally let us consider the case $\ell^{(i)}=\ell^{(i+1)}=\ell_{(i+1)}$. Then we have $P_{\ell^{(i)}}^{(i)}(\nu)=P_{\ell^{(i)}}^{(i)}(\bar{\nu})+1$ so that $P_{\ell^{(i)}}^{(i)}(\bar{\nu}) \geq-1$. Thus

$$
-P_{\ell^{(i)}-2}^{(i)}(\bar{\nu})+2 P_{\ell^{(i)}-1}^{(i)}(\bar{\nu})-P_{\ell^{(i)}}^{(i)}(\bar{\nu}) \leq-1 .
$$

In this case, we have $m_{\ell^{(i)}-1}^{(i)}(\bar{\nu})=1$ and $m_{\ell^{(i)}-1}^{(i+1)}(\bar{\nu}) \geq 2$. Thus

$$
m_{\ell^{(i)}-1}^{(i-1)}(\bar{\nu})-2 m_{\ell^{(i)}-1}^{(i)}(\bar{\nu})+m_{\ell^{(i)}-1}^{(i+1)}(\bar{\nu}) \geq 0 .
$$

This contradicts the convexity relation of Lemma 3.13.

Step 2. Let us consider the case $\ell_{(i)}<\infty$. If $\ell^{(i)}=\ell_{(i)}$ we can use the previous arguments to deduce a contradiction. Note that we have $\ell^{(i+1)}=\ell_{(i+1)}$ in this case. Thus we assume that $\ell^{(i)}<\ell_{(i)}$. Suppose that we have $\ell^{(i)}=\ell_{(i)}-1$. Suppose that we also have $\ell_{(i)}-1>\ell_{(i+1)}-1$. The full condition for this case is as follows

$$
\ell^{(i)}-1=\ell^{(i+1)}-1=\ell_{(i+1)}-1=\left(\ell_{(i)}-1\right)-1 .
$$

Thus we have $P_{\ell_{(i)}-1}^{(i)}(\nu)=P_{\ell_{(i)}-1}^{(i)}(\bar{\nu})+1$. Since the string $\left(\ell^{(i)}, P_{\ell^{(i)}}^{(i)}(\nu)\right)$ of $(\nu, J)^{(i)}$ has a nonnegative rigging, we have $P_{\ell_{(i)}-1}^{(i)}(\nu) \geq 0$. To summarize, we have $P_{\ell_{(i)}-1}^{(i)}(\bar{\nu}) \geq-1$ in this case.

1. Consider the case $P_{\ell_{(i)}-1}^{(i)}(\bar{\nu}) \geq 0$. Since $\widetilde{e}_{i}(\bar{\nu}, \bar{J}) \neq 0$ there is a string of $(\bar{\nu}, \bar{J})^{(i)}$ with negative rigging. Therefore we must have $P_{\ell^{(i)}-1}^{(i)}(\bar{\nu})<0$ since $P_{\ell_{(i)}-1}^{(i)}(\bar{\nu}) \geq 0$. Let $j$ be the largest integer such that $m_{j}^{(i)}(\bar{\nu})>0$ and $j<\ell^{(i)}-1$. If there is no such $j$, set $j=0$. Then 
we have $P_{j}^{(i)}(\bar{\nu}) \geq 0$. By the convexity relation of $P_{k}^{(i)}(\bar{\nu})$ between $j \leq k \leq \ell^{(i)}-1$, we have $P_{\ell^{(i)}-2}^{(i)}(\bar{\nu})>P_{\ell^{(i)}-1}^{(i)}(\bar{\nu})$. Since $\ell^{(i)}=\ell_{(i)}-1$, we have

$$
-P_{\ell^{(i)}-2}^{(i)}(\bar{\nu})+2 P_{\ell^{(i)}-1}^{(i)}(\bar{\nu})-P_{\ell^{(i)}}^{(i)}(\bar{\nu}) \leq-2 .
$$

On the other hand, since $\ell^{(i)}-1=\ell^{(i+1)}-1=\ell_{(i+1)}-1$ we have $m_{\ell^{(i)}-1}^{(i+1)}(\bar{\nu}) \geq 2$. Recall that the string $\left(\ell^{(i)}-1, P_{\ell^{(i)}-1}^{(i)}(\bar{\nu})\right)$ is the unique string of $(\bar{\nu}, \bar{J})^{(i)}$ with the negative rigging. Thus we have $m_{\ell^{(i)}-1}^{(i)}(\bar{\nu})=1$. Therefore we have

$$
m_{\ell^{(i)}-1}^{(i-1)}(\bar{\nu})-2 m_{\ell^{(i)}-1}^{(i)}(\bar{\nu})+m_{\ell^{(i)}-1}^{(i+1)}(\bar{\nu}) \geq 0 .
$$

This contradicts the convexity relation of Lemma 3.13.

2. Consider the case $P_{\ell_{(i)}-1}^{(i)}(\bar{\nu})=-1$ and $\ell_{(i-1)}>\ell_{(i)}$. We will show that this case cannot happen in Lemma 7.6.

3. Consider the case $P_{\ell_{(i)}-1}^{(i)}(\bar{\nu})=-1$ and $\ell_{(i-1)}=\ell_{(i)}$. We will show that this case cannot happen in Lemma 7.7.

On the other hand, together with the present assumption $\ell^{(i)}=\ell_{(i)}-1$, suppose that we also have $\ell_{(i)}-1=\ell_{(i+1)}-1$. We will show that this case cannot happen in Lemma 7.8.

Finally let us assume that $\ell^{(i)}<\ell_{(i)}-1$. Recall that the only strings of $(\bar{\nu}, \bar{J})^{(i)}$ which may have negative riggings are $\left(\ell^{(i)}-1, P_{\ell^{(i)}-1}^{(i)}(\bar{\nu})\right)$ and $\left(\ell_{(i)}-1, P_{\ell_{(i)}-1}^{(i)}(\bar{\nu})\right)$. Suppose that we have $P_{\ell^{(i)}-1}^{(i)}(\bar{\nu})<0$. In this case, as in Step 1 , we have $P_{\ell^{(i)}-2}^{(i)}(\bar{\nu})>P_{\ell^{(i)}-1}^{(i)}(\bar{\nu})$ and $P_{\ell^{(i)}}^{(i)}(\nu) \geq 0$. Then by the same arguments of Step 1 we can deduce a contradiction. Thus suppose that we have $P_{\ell^{(i)}-1}^{(i)}(\bar{\nu}) \geq 0$ in the following. Then by the assumption $\widetilde{e}_{i}(\bar{\nu}, \bar{J}) \neq 0$, we have $P_{\ell_{(i)}-1}^{(i)}(\bar{\nu})<0$. Let $j$ be the largest integer such that $j<\ell_{(i)}-1$ and $m_{j}^{(i)}(\bar{\nu})>0$. Then we have $P_{j}^{(i)}(\bar{\nu}) \geq 0$ and by the convexity relation of $P_{k}^{(i)}(\bar{\nu})$ between $j \leq k \leq \ell_{(i)}-1$, we have $P_{\ell_{(i)}-2}^{(i)}(\bar{\nu})>P_{\ell_{(i)}-1}^{(i)}(\bar{\nu})$. On the other hand, from the assumption $\widetilde{e}_{i}(\nu, J)=0$ we see that the string $\left(\ell_{(i)}, P_{\ell_{(i)}}^{(i)}(\nu)\right)$ of $(\nu, J)^{(i)}$ must have a non-negative rigging. Thus we have $P_{\ell_{(i)}}^{(i)}(\nu) \geq 0$. Then we can use the same arguments of Step 1 if we replace $\ell^{(i)}$ and $\ell^{(i+1)}$ there by $\ell_{(i)}$ and $\ell_{(i-1)}$ respectively to deduce a contradiction. Thus we have confirmed that the final case $\ell^{(i)}<\ell_{(i)}-1$ cannot happen.

Lemma 7.6. The following situation cannot happen. $\widetilde{e}_{i}(\nu, J)=0, \widetilde{e}_{i}(\bar{\nu}, \bar{J}) \neq 0$,

$$
\ell^{(i)}-1=\ell^{(i+1)}-1=\ell_{(i+1)}-1=\left(\ell_{(i)}-1\right)-1,
$$

$\ell_{(i-1)}>\ell_{(i)}$ and $P_{\ell_{(i)}-1}^{(i)}(\bar{\nu})=-1$.

Proof. Since $P_{\ell_{(i)}}^{(i)}(\nu)=P_{\ell_{(i)}}^{(i)}(\bar{\nu})-1$, we have $P_{\ell_{(i)}}^{(i)}(\bar{\nu}) \geq 1$ by $\widetilde{e}_{i}(\nu, J)=0$. Let us show that $m_{\ell_{(i)}-1}^{(i)}(\bar{\nu})=1$. Since we know that $\ell^{(i)}-1<\ell_{(i)}-1$, we see that $\delta^{-1}$ will add boxes to the strings $\left(\ell^{(i)}-1, P_{\ell^{(i)}-1}^{(i)}(\bar{\nu})\right)$ and $\left(\ell_{(i)}-1, P_{\ell_{(i)}-1}^{(i)}(\bar{\nu})\right)$. Thus if we have $m_{\ell_{(i)}-1}^{(i)}(\bar{\nu})>1$, there will be a remaining negative rigging in $(\nu, J)^{(i)}$ by $P_{\ell_{(i)}-1}^{(i)}(\bar{\nu})<0$, which is in contradiction to the assumption $\widetilde{e}_{i}(\nu, J)=0$. Therefore we have $m_{\ell_{(i)}-1}^{(i)}(\bar{\nu})=1$. Then $P_{\ell_{(i)}-2}^{(i)}(\bar{\nu}), P_{\ell_{(i)}-1}^{(i)}(\bar{\nu})$ and $\left(P_{\ell_{(i)}}^{(i)}(\bar{\nu})-2\right)$ must satisfy the convexity relation by Lemma 3.12. Since $P_{\ell_{(i)}-1}^{(i)}(\bar{\nu})=-1$ and $P_{\ell_{(i)}}^{(i)}(\bar{\nu}) \geq 1$, we see that

$$
P_{\ell_{(i)}-2}^{(i)}(\bar{\nu})=P_{\ell^{(i)}-1}^{(i)}(\bar{\nu}) \leq-1 .
$$


From this, we also have $m_{\ell^{(i)}-1}^{(i)}(\bar{\nu})=1$ as otherwise we would have a remaining negative rigging in $(\nu, J)^{(i)}$. Let $j$ be the largest integer such that $m_{j}^{(i)}(\bar{\nu})>0$ and $j<\ell^{(i)}-1$. If there is no such $j$, set $j=0$. Then we have $P_{j}^{(i)}(\bar{\nu}) \geq 0$ since the only strings of $(\bar{\nu}, \bar{J})^{(i)}$ with negative riggings are $\left(\ell^{(i)}-1, P_{\ell^{(i)}-1}^{(i)}(\bar{\nu})\right)$ and $\left(\ell_{(i)}-1, P_{\ell_{(i)}-1}^{(i)}(\bar{\nu})\right)$. Then from the convexity relation of $P_{k}^{(i)}(\bar{\nu})$ between $j \leq k \leq \ell^{(i)}-1$ and $(7.2)$, we have

$$
P_{\ell^{(i)}-2}^{(i)}(\bar{\nu})>P_{\ell^{(i)}-1}^{(i)}(\bar{\nu}) .
$$

Then the left hand side of the convexity relation of Lemma 3.13 is

$$
-P_{\ell^{(i)}-2}^{(i)}(\bar{\nu})+2 P_{\ell^{(i)}-1}^{(i)}(\bar{\nu})-P_{\ell^{(i)}}^{(i)}(\bar{\nu}) \leq-1
$$

by $\ell_{(i)}-1=\ell^{(i)}$ and the right hand side of it is

$$
m_{\ell^{(i)}-1}^{(i-1)}(\bar{\nu})-2 m_{\ell^{(i)}-1}^{(i)}(\bar{\nu})+m_{\ell^{(i)}-1}^{(i+1)}(\bar{\nu}) \geq 0 .
$$

Here we use the fact $m_{\ell^{(i)}-1}^{(i+1)}(\bar{\nu}) \geq 2$ which follows from $\ell^{(i)}-1=\ell^{(i+1)}-1=\ell_{(i+1)}-1$. This is a contradiction.

Lemma 7.7. The following situation cannot happen. $\widetilde{e}_{i}(\nu, J)=0, \widetilde{e}_{i}(\bar{\nu}, \bar{J}) \neq 0$,

$$
\ell^{(i)}-1=\ell^{(i+1)}-1=\ell_{(i+1)}-1=\left(\ell_{(i)}-1\right)-1=\left(\ell_{(i-1)}-1\right)-1
$$

and $P_{\ell_{(i)}-1}^{(i)}(\bar{\nu})=-1$

Proof. Since $P_{\ell_{(i)}}^{(i)}(\nu)=P_{\ell_{(i)}}^{(i)}(\bar{\nu})$, we have

$$
P_{\ell_{(i)}}^{(i)}(\bar{\nu}) \geq 0
$$

by $\widetilde{e}_{i}(\nu, J)=0$. As in Lemma 7.6, we have $m_{\ell_{(i)}-1}^{(i)}(\bar{\nu})=1$ by $\ell^{(i)}-1<\ell_{(i)}-1$. If we use $P_{\ell_{(i)}-1}^{(i)}(\bar{\nu})=-1$, the left hand side of the convexity relation of Lemma 3.13 with $l=\ell_{(i)}-1$ is

$$
-P_{\ell_{(i)}-2}^{(i)}(\bar{\nu})+2 P_{\ell_{(i)}-1}^{(i)}(\bar{\nu})-P_{\ell_{(i)}}^{(i)}(\bar{\nu}) \leq-P_{\ell_{(i)}-2}^{(i)}(\bar{\nu})-2
$$

and the right hand side of it is

$$
m_{\ell_{(i)}-1}^{(i-1)}(\bar{\nu})-2 m_{\ell_{(i)}-1}^{(i)}(\bar{\nu})+m_{\ell_{(i)}-1}^{(i+1)}(\bar{\nu}) \geq-1+m_{\ell_{(i)}-1}^{(i+1)}(\bar{\nu}) \geq-1 .
$$

Here we have used $m_{\ell_{(i)}-1}^{(i-1)}(\bar{\nu}) \geq 1$ by $\ell_{(i-1)}-1=\ell_{(i)}-1$. Therefore we have

$$
P_{\ell_{(i)}-2}^{(i)}(\bar{\nu})=P_{\ell^{(i)}-1}^{(i)}(\bar{\nu}) \leq-1 .
$$

From this and $P_{\ell_{(i)}-1}^{(i)}(\bar{\nu})=-1$ we deduce that $m_{\ell^{(i)}-1}^{(i)}(\bar{\nu})=1$ as otherwise we must have $\widetilde{e}_{i}(\nu, J) \neq 0$. Let $j$ be the largest integer such that $m_{j}^{(i)}(\bar{\nu})>0$ and $j<\ell^{(i)}-1$. If there is no such $j$, set $j=0$. Then we have $P_{j}^{(i)}(\bar{\nu}) \geq 0$ since the only strings of $(\bar{\nu}, \bar{J})^{(i)}$ which may have negative riggings are $\left(\ell^{(i)}-1, P_{\ell^{(i)}-1}^{(i)}(\bar{\nu})\right)$ and $\left(\ell_{(i)}-1, P_{\ell_{(i)}-1}^{(i)}(\bar{\nu})\right)$. Then by the convexity relation of $P_{k}^{(i)}(\bar{\nu})$ between $j \leq k \leq \ell^{(i)}-1$ we have

$$
P_{\ell^{(i)}-2}^{(i)}(\bar{\nu})>P_{\ell^{(i)}-1}^{(i)}(\bar{\nu}) .
$$


Recall that the left hand side of the convexity relation of Lemma 3.13 with $l=\ell^{(i)}-1$ is

$$
-P_{\ell^{(i)}-2}^{(i)}(\bar{\nu})+2 P_{\ell^{(i)}-1}^{(i)}(\bar{\nu})-P_{\ell^{(i)}}^{(i)}(\bar{\nu}) \leq P_{\ell^{(i)}-1}^{(i)}(\bar{\nu})
$$

by $P_{\ell^{(i)}-2}^{(i)}(\bar{\nu})>P_{\ell^{(i)}-1}^{(i)}(\bar{\nu})$ and $P_{\ell^{(i)}}^{(i)}(\bar{\nu})=-1$ by $\ell^{(i)}=\ell_{(i)}-1$. On the other hand, the right hand side of the convexity relation of Lemma 3.13 is

$$
m_{\ell^{(i)}-1}^{(i-1)}(\bar{\nu})-2 m_{\ell^{(i)}-1}^{(i)}(\bar{\nu})+m_{\ell^{(i)}-1}^{(i+1)}(\bar{\nu})=m_{\ell^{(i)}-1}^{(i-1)}(\bar{\nu})-2+m_{\ell^{(i)}-1}^{(i+1)}(\bar{\nu}) \geq m_{\ell^{(i)}-1}^{(i-1)}(\bar{\nu}) .
$$

Here we use the fact $m_{\ell^{(i)}-1}^{(i+1)}(\bar{\nu}) \geq 2$ which follows from $\ell^{(i)}-1=\ell^{(i+1)}-1=\ell_{(i+1)}-1$. This is a contradiction since we have (7.3).

Lemma 7.8. The following situation cannot happen. $\widetilde{e}_{i}(\nu, J)=0, \widetilde{e}_{i}(\bar{\nu}, \bar{J}) \neq 0$ and

$$
\ell^{(i)}-1=\left(\ell_{(i+1)}-1\right)-1=\left(\ell_{(i)}-1\right)-1 .
$$

Proof. In this case we have $P_{\ell_{(i)}-1}^{(i)}(\nu) \leq P_{\ell_{(i)}}^{(i)}(\bar{\nu})$. Suppose if possible that we have $P_{\ell_{(i)}-1}^{(i)}(\bar{\nu})<0$. Then the string $\left(\ell_{(i)}-1, P_{\ell_{(i)}-1}^{(i)}(\nu)\right)$ of $(\nu, J)^{(i)}$ has a negative rigging. This is a contradiction since we have $\widetilde{e}_{i}(\nu, J)=0$. Thus we can assume that $P_{\ell_{(i)}-1}^{(i)}(\bar{\nu})=P_{\ell^{(i)}}^{(i)}(\bar{\nu}) \geq 0$ by $\ell^{(i)}=\ell_{(i)}-1$. Then we see that we must have

$$
P_{\ell^{(i)}-1}^{(i)}(\bar{\nu})<0
$$

since we have $\widetilde{e}_{i}(\bar{\nu}, \bar{J}) \neq 0$.

Let $j$ be the largest integer such that $j<\ell^{(i)}-1$ and $m_{j}^{(i)}(\bar{\nu})>0$. If there is no such string, set $j=0$. Then we have $P_{j}^{(i)}(\bar{\nu}) \geq 0$. From the convexity relation of $P_{k}^{(i)}(\bar{\nu})$ between $j \leq k \leq \ell^{(i)}-1$, we have

$$
P_{\ell^{(i)}-2}^{(i)}(\bar{\nu})>P_{\ell^{(i)}-1}^{(i)}(\bar{\nu})
$$

since $P_{\ell^{(i)}-1}^{(i)}(\bar{\nu})<0$.

Let us show that $m_{\ell^{(i)}-1}^{(i)}(\bar{\nu})=1$. Suppose if possible that we have $m_{\ell^{(i)}-1}^{(i)}(\bar{\nu})>1$. Since we have $P_{\ell^{(i)}-1}^{(i)}(\bar{\nu})<0$ and $\ell^{(i)}-1<\ell_{(i)}-1$, we see that there is the remaining negative rigging in $(\nu, J)^{(i)}$. This is a contradiction since we have $\widetilde{e}_{i}(\nu, J)=0$. Thus we have $m_{\ell^{(i)}-1}^{(i)}(\bar{\nu})=1$.

Case 1. Suppose that we have $\ell^{(i)}-1=\left(\ell^{(i+1)}-1\right)-1$. Then we have $P_{\ell^{(i)}}^{(i)}(\nu)=P_{\ell^{(i)}}^{(i)}(\bar{\nu})-1$. From $\widetilde{e}_{i}(\nu, J)=0$ we have $P_{\ell^{(i)}}^{(i)}(\bar{\nu}) \geq 1$. To summarize, we have

$$
P_{\ell^{(i)}-2}^{(i)}(\bar{\nu})>P_{\ell^{(i)}-1}^{(i)}(\bar{\nu})<0, \quad P_{\ell^{(i)}}^{(i)}(\bar{\nu}) \geq 1 .
$$

Let $n_{l}^{(a)}$ be the length of the $l$ th column of $\bar{\nu}^{(a)}$

$$
n_{l}^{(a)}:=\sum_{l \leq k} m_{k}^{(a)}(\bar{\nu})
$$

Then the above relations imply that

$$
n_{\ell^{(i)}-1}^{(i-1)}-2 n_{\ell^{(i)}-1}^{(i)}+n_{\ell^{(i)}-1}^{(i+1)} \leq-1, \quad n_{\ell^{(i)}}^{(i-1)}-2 n_{\ell^{(i)}}^{(i)}+n_{\ell^{(i)}}^{(i+1)} \geq 2 .
$$


Since we have $m_{\ell^{(i)}-1}^{(i)}(\bar{\nu})=1$, we have $n_{\ell^{(i)}}^{(i)}=n_{\ell^{(i)}-1}^{(i)}-1$ here. Then the second relation of (7.4) becomes $n_{\ell^{(i)}}^{(i-1)}-2 n_{\ell^{(i)}-1}^{(i)}+n_{\ell^{(i)}}^{(i+1)} \geq 0$. Combining this with the first relation of (7.4), we obtain

$$
0>\left(n_{\ell^{(i)}-1}^{(i-1)}-n_{\ell^{(i)}}^{(i-1)}\right)+\left(n_{\ell^{(i)}-1}^{(i+1)}-n_{\ell^{(i)}}^{(i+1)}\right) .
$$

This is a contradiction since we have $n_{l}^{(a)} \geq n_{l+1}^{(a)}$ by definition of $n_{l}^{(a)}$.

Case 2. Suppose that we have $\ell^{(i)}-1=\ell^{(i+1)}-1$. In particular, we have $m_{\ell^{(i)}-1}^{(i+1)}(\bar{\nu})>0$. As in the previous case, we have

$$
P_{\ell^{(i)}-2}^{(i)}(\bar{\nu})>P_{\ell^{(i)}-1}^{(i)}(\bar{\nu})<0, \quad P_{\ell^{(i)}}^{(i)}(\bar{\nu}) \geq 0 .
$$

Therefore we have

$$
n_{\ell^{(i)}-1}^{(i-1)}-2 n_{\ell^{(i)}-1}^{(i)}+n_{\ell^{(i)}-1}^{(i+1)} \leq-1, \quad n_{\ell^{(i)}}^{(i-1)}-2 n_{\ell^{(i)}}^{(i)}+n_{\ell^{(i)}}^{(i+1)} \geq 1 .
$$

From $n_{\ell^{(i)}}^{(i)}=n_{\ell^{(i)}-1}^{(i)}-1$, the second relation of (7.5) gives $n_{\ell^{(i)}}^{(i-1)}-2 n_{\ell^{(i)}-1}^{(i)}+n_{\ell^{(i)}}^{(i+1)} \geq-1$. Then from the first relation of $(7.5)$, we obtain

$$
0 \geq\left(n_{\ell^{(i)}-1}^{(i-1)}-n_{\ell^{(i)}}^{(i-1)}\right)+\left(n_{\ell^{(i)}-1}^{(i+1)}-n_{\ell^{(i)}}^{(i+1)}\right) .
$$

Therefore we have $n_{\ell^{(i)}-1}^{(i-1)}=n_{\ell^{(i)}}^{(i-1)}$ and $n_{\ell^{(i)}-1}^{(i+1)}=n_{\ell^{(i)}}^{(i+1)}$. In particular, we have $m_{\ell^{(i)}-1}^{(i+1)}(\bar{\nu})=0$, which is a contradiction. Hence this case cannot happen.

\subsection{Proof for (8)}

Proposition 7.9. Let $b=b^{\prime} \otimes \bar{b} \in B^{1,1} \otimes \bar{B}$ and $i \in I_{0}$. Then the situation $\widetilde{e}_{i}(b)=0$ and $\widetilde{e}_{i}(\bar{b}) \neq 0$ cannot happen.

Proof. The proof of this assertion follows from the tensor product rule at Section 2.1.

\section{Comments on Deka-Schilling's work}

In Appendix C of the original preprint version of [4], Deka and Schilling proved the compatibility of the classical Kashiwara operators and the rigged configuration bijection of type $A_{n}^{(1)}$. I am pleased to admit that they proved some portion of the entire proof and also I would like to sincerely admire that they embarked on such a difficult problem. However, due to the following crucial problems, the author assumes that they did not finish their proof.

(a) In [4], the proofs for $\widetilde{e}_{i}$ case are completely omitted since they claim at Lemmas C.2 and C.3 (i.e., not in the main proof) that the proofs are "similar" to $\widetilde{f}_{i}$ case. Actually, as we have shown in Sections 6 and 7 of the present paper, the logical structure of two proofs are entirely different. In particular, the proofs for $\widetilde{e}_{i}$ case require the result of $\widetilde{f}_{i}$ case which was proved beforehand. It is natural as the statements of the necessary propositions are already different.

(b) In [4], the authors give entirely no words about the riggings. As we have shown in the present paper, the coincidence of the riggings is non-trivial in many cases since the Kashiwara operators on the rigged configurations modify riggings. Thus they strongly deserve individual and careful analysis.

(c) In [4], the authors give no words to Cases A and E of the present paper. They simply claim that the only non-trivial cases are the Cases B, C and D. However, as we have shown in the present paper, each proof of Case $\mathrm{A}$ or $\mathrm{E}$ breaks into subcases and requires somewhat elaborated arguments even if we restrict to the type $A_{n}^{(1)}$. Thus it is necessary to mention about these cases. 


\section{Acknowledgements}

The author would like to thank Professor Masato Okado for valuable discussion throughout the project. In particular, the project began when both of us worked together to understand the details of Appendix $\mathrm{C}$ of the preprint version of [4]. The author is also grateful to Professor Anne Schilling for valuable discussion on her results. For both of them, the author is very grateful for the fruitful collaboration on closely related projects $[24,25]$ which provides a motivation and an important application of the present work. I would like to thank anonymous referees for valuable suggestions which greatly help the author to improve the original manuscript. This work is partially supported by Grants-in-Aid for Scientific Research No. 21740114 from JSPS.

\section{References}

[1] Alba V.A., Fateev V.A., Litvinov A.V., Tarnopolskiy G.M., On combinatorial expansion of the conformal blocks arising from AGT conjecture, Lett. Math. Phys. 98 (2011), 33-64, arXiv:1012.1312.

[2] Belavin A., Belavin V., AGT conjecture and integrable structure of conformal field theory for $c=1, N u c l e a r$ Phys. B 850 (2011), 199-213, arXiv:1102.0343.

[3] Cai W., Jing N., Applications of a Laplace-Beltrami operator for Jack polynomials, European J. Combin. 33 (2012), 556-571, arXiv:1101.5544.

[4] Deka L., Schilling A., New fermionic formula for unrestricted Kostka polynomials, J. Combin. Theory Ser. A 113 (2006), 1435-1461, math.CO/0509194.

[5] Desrosiers P., Lapointe L., Mathieu P., Superconformal field theory and Jack superpolynomials, J. High Energy Phys. 2012 (2012), no. 9, 037, 42 pages, arXiv:1205.0784.

[6] Estienne B., Pasquier V., Santachiara R., Serban D., Conformal blocks in Virasoro and W theories: duality and the Calogero-Sutherland model, Nuclear Phys. B 860 (2012), 377-420, arXiv:1110.1101.

[7] Feigin B.L., Fuchs D.B., Representations of the Virasoro algebra, in Representation of Lie Groups and Related Topics, Adv. Stud. Contemp. Math., Vol. 7, Gordon and Breach, New York, 1990, 465-554.

[8] Feigin M.V., Hallnäs M.A., Veselov A.P., Baker-Akhiezer functions and generalised Macdonald-Mehta integrals, J. Math. Phys. 54 (2013), 052106, 22 pages, arXiv:1210.5270.

[9] Fukuda K., Yamada Y., Okado M., Energy functions in box ball systems, Internat. J. Modern Phys. A 15 (2000), 1379-1392, math.QA/9908116.

[10] Hatayama G., Hikami K., Inoue R., Kuniba A., Takagi T., Tokihiro T., The $A_{M}^{(1)}$ automata related to crystals of symmetric tensors, J. Math. Phys. 42 (2001), 274-308, math.QA/9912209.

[11] Hatayama G., Kuniba A., Takagi T., Soliton cellular automata associated with crystal bases, Nuclear Phys. B 577 (2000), 619-645, solv-int/9907020.

[12] Hatayama G., Kuniba A., Takagi T., Simple algorithm for factorized dynamics of the $\mathfrak{g}_{n}$-automaton, J. Phys. A: Math. Gen. 34 (2001), 10697-10705, nlin.CG/0103022.

[13] Kac V.G., Infinite-dimensional Lie algebras, 3rd ed., Cambridge University Press, Cambridge, 1990.

[14] Kashiwara M., On crystal bases of the Q-analogue of universal enveloping algebras, Duke Math. J. 63 (1991), $465-516$.

[15] Kashiwara M., Bases cristallines des groupes quantiques, Cours Spécialisés, Vol. 9, Société Mathématique de France, Paris, 2002.

[16] Kashiwara M., Nakashima T., Crystal graphs for representations of the $q$-analogue of classical Lie algebras, J. Algebra 165 (1994), 295-345.

[17] Kerov S.V., Kirillov A.N., Reshetikhin N.Y., Combinatorics, the Bethe ansatz and representations of the symmetric group, J. Soviet Math. 41 (1988), 916-924.

[18] Kirillov A.N., Reshetikhin N.Y., The Bethe ansatz and the combinatorics of Young tableaux, J. Soviet Math. 41 (1988), 925-955.

[19] Kirillov A.N., Schilling A., Shimozono M., A bijection between Littlewood-Richardson tableaux and rigged configurations, Selecta Math. (N.S.) 8 (2002), 67-135, math.CO/9901037.

[20] Kuniba A., Okado M., Sakamoto R., Takagi T., Yamada Y., Crystal interpretation of Kerov-KirillovReshetikhin bijection, Nuclear Phys. B $\mathbf{7 4 0}$ (2006), 299-327, math.QA/0601630. 
[21] Kuniba A., Sakamoto R., Yamada Y., Generalized energies and integrable $D_{n}^{(1)}$ cellular automaton, in New Trends in Quantum Integrable Systems, World Sci. Publ., Hackensack, NJ, 2011, 221-242, arXiv:1001.1813.

[22] Mimachi K., Yamada Y., Singular vectors of the Virasoro algebra in terms of Jack symmetric polynomials, Comm. Math. Phys. 174 (1995), 447-455.

[23] Okado M., Sakamoto R., Stable rigged configurations for quantum affine algebras of nonexceptional types, Adv. Math. 228 (2011), 1262-1293, arXiv:1008.0460.

[24] Okado M., Sakamoto R., Schilling A., Affine crystal structure on rigged configurations of type $D_{n}^{(1)}, J$. Algebraic Combin. 37 (2013), 571-599, arXiv:1109.3523.

[25] Okado M., Sakamoto R., Schilling A., Kerov-Kirillov-Reshetikhin type bijection for $D_{n}^{(1)}$, in preparation.

[26] Okado M., Sano N., KKR type bijection for the exceptional affine algebra $E_{6}^{(1)}$, in Algebraic Groups and Quantum Groups, Contemp. Math., Vol. 565, Amer. Math. Soc., Providence, RI, 2012, 227-242, arXiv:1105.1636.

[27] Okado M., Schilling A., Shimozono M., A crystal to rigged configuration bijection for nonexceptional affine algebras, in Algebraic Combinatorics and Quantum Groups, World Sci. Publ., River Edge, NJ, 2003, 85-124, math.QA/0203163.

[28] Sakamoto R., Kirillov-Schilling-Shimozono bijection as energy functions of crystals, Int. Math. Res. Not. 2009 (2009), 579-614, arXiv:0711.4185.

[29] Sakamoto R., Ultradiscrete soliton systems and combinatorial representation theory, arXiv:1212.2774.

[30] Sakamoto R., Shiraishi J., Arnaudon D., Frappat L., Ragoucy E., Correspondence between conformal field theory and Calogero-Sutherland model, Nuclear Phys. B 704 (2005), 490-509, hep-th/0407267.

[31] Schilling A., A bijection between type $D_{n}^{(1)}$ crystals and rigged configurations, J. Algebra 285 (2005), 292-334, math.QA/0406248.

[32] Schilling A., Crystal structure on rigged configurations, Int. Math. Res. Not. 2006 (2006), Art. ID 97376, 27 pages, math.QA/0508107.

[33] Schilling A., Combinatorial structure of Kirillov-Reshetikhin crystals of type $D_{n}^{(1)}, B_{n}^{(1)}, A_{2 n-1}^{(2)}, J$. Algebra 319 (2008), 2938-2962, arXiv:0704.2046.

[34] Schilling A., Shimozono M., $X=M$ for symmetric powers, J. Algebra 295 (2006), 562-610, math.QA/0412376.

[35] Sutherland B., Exact results for a quantum many-body problem in one dimension, Phys. Rev. A 4 (1971), 2019-2021.

[36] Sutherland B., Exact results for a quantum many-body problem in one dimension. II, Phys. Rev. A 5 (1972), 1372-1376.

[37] Takahashi D., Satsuma J., A soliton cellular automaton, J. Phys. Soc. Japan 59 (1990), 3514-3519. 